\title{
Tumour hypoxia
}

Citation for published version (APA):

Keulers, T. G. H. (2019). Tumour hypoxia: effects on autophagy and intercellular communication.

[Doctoral Thesis, Maastricht University]. ProefschriftMaken Maastricht.

https://doi.org/10.26481/dis.20190201tk

Document status and date:

Published: 01/01/2019

DOI:

10.26481/dis.20190201tk

Document Version:

Publisher's PDF, also known as Version of record

\section{Please check the document version of this publication:}

- A submitted manuscript is the version of the article upon submission and before peer-review. There can be important differences between the submitted version and the official published version of record.

People interested in the research are advised to contact the author for the final version of the publication, or visit the DOI to the publisher's website.

- The final author version and the galley proof are versions of the publication after peer review.

- The final published version features the final layout of the paper including the volume, issue and page numbers.

Link to publication

\footnotetext{
General rights rights.

- You may freely distribute the URL identifying the publication in the public portal. please follow below link for the End User Agreement:

www.umlib.nl/taverne-license

Take down policy

If you believe that this document breaches copyright please contact us at:

repository@maastrichtuniversity.nl

providing details and we will investigate your claim.
}

Copyright and moral rights for the publications made accessible in the public portal are retained by the authors and/or other copyright owners and it is a condition of accessing publications that users recognise and abide by the legal requirements associated with these

- Users may download and print one copy of any publication from the public portal for the purpose of private study or research.

- You may not further distribute the material or use it for any profit-making activity or commercial gain

If the publication is distributed under the terms of Article $25 \mathrm{fa}$ of the Dutch Copyright Act, indicated by the "Taverne" license above, 


\section{Tumour hypoxia effects on autophagy and intercellular communication}




\section{Tumour hypoxia}

\section{Effects on autophagy and intercellular communication}


(C) copyright Tom Gertrudis Hubertus Keulers, Maastricht 2019

Printing: ProefschriftMaken || www.proefschriftmaken.nl

ISBN 978-94-6380-175-1

All rights reserved. No part of this publication may be reproduced, stored in a retrieval system or transmitted, in any form or by any means, electronic, mechanical, photocopying, recording or otherwise, without prior permission of the author or the copyright-owning journals for previous published chapters. 


\title{
Tumour hypoxia
}

\section{Effects on autophagy and intercellular communication}

\author{
DISSERTATION \\ to obtain the degree of Doctor at Maastricht University, \\ on the authority of the Rector Magnificus, Prof. Dr. Rianne M. Letschert \\ in accordance with the decision of the Board of Deans, \\ to be defended in public \\ on Friday February $1^{\text {st }} 2019$ at $14: 00$ hours \\ by \\ Tom Gertrudis Hubertus Keulers
}




\section{Supervisors}

Dr. K.M.A. Rouschop

Prof. dr. M.A.G.G Vooijs

\section{Assessment Committee}

Prof. dr. F.C.S. Ramaekers (Voorzitter)

Dr. E.N.M. Nolte-'t Hoen (Utrecht University)

Prof. dr. R.M. Schiffelers (Utrecht University)

Prof. dr. P. Schrauwen

Dr. L. Wieten 


\section{Contents}

Chapter 1 Introduction

Chapter 2 LC3/GABARAP family proteins: Autophagy (un)related functions

Chapter 3 GABARAPL1 is required for increased EGFR membrane expression during hypoxia

Chapter 4 EGFRvIII expression triggers a metabolic dependency and therapeutic vulnerability sensitive to autophagy inhibition

Chapter 5 ATG12 expression predicts tumour hypoxia and tumour control in HNSCC

Chapter 6 Autophagy-dependent secretion: contribution to tumour progression

Chapter 7 GABARAPL1 is required for the secretion of pro-angiogenic extracellular vesicles during hypoxia

Chapter 8 GABARAPL1 is essential for metastasis formation in mice

Chapter 9 Summary and general discussion

Nederlandse samenvatting 195

Valorisation

Acknowledgments / Dankwoord 205

Curriculum Vitae 209

List of Publications 



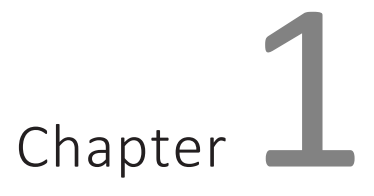

Introduction 


\section{Introduction}

Cancer is a devastating disease that 1 in 3 people in their lifetime are faced with. In Europe, 3.7 million new cases and 1.9 million deaths are reported annually, which makes cancer the second most important cause of death and morbidity (1). Due to the aging of our population this incidence will increase in the coming decades. Although many advances in treatment of cancer have been made, novel therapeutic strategies and further understanding of the complexity of the disease and mechanisms of disease progression are highly needed to further increase the therapeutic effectiveness and cure of patients.

Cancer originates from cells that have lost the control over cell proliferation and cell death. Solid tumours evolve into complex tissues that contain, in addition to cancer cells, other recruited cell types including cells of the immune system, the tumour bloodand lymphatic vasculature and fibroblasts. Together with extracellular matrix this creates the tumour microenvironment (TME). Cells of cancerous and non-cancerous origin in the TME constantly interact and thereby contribute to development of the tumour. The TME of solid tumours is characterized by poorly oxygenated regions (hypoxia) and regions that receive limited nutrients. Hypoxia occurs when oxygen demand exceeds delivery due to alterations of the metabolic and proliferative status of tumour cells or as a result of insufficient blood delivery due to an immature and chaotic tumour vasculature. In general, two distinct patterns of hypoxia are considered. 1) Diffusion-limited, or chronic, hypoxia which is caused by limitations in oxygen diffusion from the tumour vasculature in the surrounding tissue or increased $\mathrm{O}_{2}$ consumption rates. Typically, cellular proliferation results in increased distance of cells to the nearest perfused vessel. Oxygen consumption by cells closer to the vessel results in reduced delivery and creates oxygen gradients in relation to distance from the vessel. During diffusion-limited or chronic hypoxia, oxygen levels vary at a relatively slow rate (hours to days). 2) Perfusion-limited, or acute hypoxia is caused by temporal occlusion of vessels and leads to rapid fluctuations between oxygenation (minutes to hours)(2). Typically, normal tissues vary between $3.9 \%$ and $6.8 \% \mathrm{O}_{2}(3)$. In cancer this is much more diverse. For example in breast (4), cervix (5), head and neck (6) and prostate carcinomas (7), oxygen levels vary between normal oxygenation to complete anoxia.

Tumour hypoxia is a driver for selection of cells that have lost their apoptotic potential, as firstly described by Greaber et al. (8) who showed that p53 deficient cells take over the population under the pressure of hypoxia. From a clinical point of view, means of reducing the hypoxic fraction of tumours is highly desired since low oxygenation of tumours is associated with poor outcome in multiple cancer types, independent of treatment modality $(9,10)$. In HNSCC, where a meta-analysis indicated that the degree of hypoxia is the most significant factor explaining variability in survival (11), the evidence is strongest. The observed effect on local control is most likely caused by in sensitivity of hypoxic cells to both chemo- and radiotherapy. Additionally there is an associa- 
tion between hypoxia and the occurrence of metastasis (12). It has been proposed that tumour hypoxia contributes to malignancy e.g. through activation of epithelial to mesenchymal transition (13-16), stimulation of blood vessel growth and formation of the pre-metastatic niche through secretion of microvesicles/exosomes/extracellular vesicles (hereafter indicated by EV) (17-25). Importantly, in head and neck squamous cell carcinoma (HNSCC) evidence of the therapeutic benefit of hypoxia modification is strongest (26), indicating that hypoxia modification does not only influence local control, but also overall outcome.

In short, tumour hypoxia leads to the expansion of cells with a more invasive, metastatic and therapy resistant phenotype. Clinical studies show that hypoxia contributes to poor clinical outcome as it decreases loco-regional control, decreases disease freesurvival and overall survival in head and neck squamous cell carcinoma (HNSCC) (27) (28). Therefore it is essential to understand hypoxia and associated processes in order to selectively target these areas.

\section{Hypoxia responsive pathways}

In response to hypoxia, cells activate oxygen sensitive pathways to adapt and survive these severe conditions. The hypoxia inducible factor (HIF) pathway is the most investigated hypoxia-responsive pathway. HIF are heterodimers composed of an oxygenregulated subunit (HIF1 $\alpha, 2 \alpha$ and $3 \alpha$, collectively referred to as HIF $\alpha$ ) and $\$$ (ubunit (HIF1 $\beta$ /ARNT1 , ARNT2 and ARNT3 ). When oxygen is present, HIF1 $\alpha$ undergeesmatic hydroxylation by the $\mathrm{O}_{2}$ regulated prolyl-4-hydroxylases (PHDs) and will be degraded by the proteasome after binding by the von Hippel-Lindau ( $\mathrm{VVHL}$ ) tumour suppressor. In contrast, during hypoxia, PHD enzymes are inhibited and HIF1 a hydroxylation leads to HIF1 a stabilization leading to transcriptional activation of target genes after bing to hypoxia responsive elements (HRE) in the promoter regions. HIF activation is associated with enhanced angiogenesis by secretion of growth factors such as VEGF, altered metabolism by the regulation of glycolytic genes to promote glycolysis, migration by activating the Wnt/ $\beta$-catenin or PI3K/AKT pathway, invasion and metastasis (reviewed in (29)).

During severe hypoxia $\left(<0.2 \% \mathrm{O}_{2}\right)$, the folding of proteins in the endoplasmic reticulum (ER) is compromised and misfolded proteins accumulate in the ER (30). As a result, the unfolded protein Response (UPR) is activated. Activation of the UPR is initiated by three ERtransmembrane stress sensors: Activating Transcription Factor 6 (ATF6), PKR)-like endoplasmic reticulum kinase (PERK) and Serine/threonine-protein kinase/endoribonuclease (IRE1). Typically these sensors are kept in an inactivated state through binding to heat shock protein family A (Hsp70) member 5 (HSPA5/ also known as BiP/GRP78). Due to the higher affinity of BiP to unfolded proteins, accumulation of unfolded proteins results in BiPdissociation from the sensor and thereby activating them. Activation of the UPR leads to decreased overall protein synthesis, translation of specific proteins, increased protein traf- 
ficking through the ER and increased protein degradation by ER-associated degradation (ERAD) and autophagy.

Autophagy is a cellular degradation- and recycle mechanism to maintain homeostasis of the cell and achieves this by recycling damaged and aged cytoplasmic content, such as aggregated or misfolded proteins and organelles. In mice, autophagy enables survival to neonatal starvation by preventing energy depletion (31). Furthermore, defective autophagy in brain causes accumulation of damaged mitochondria and protein aggregates and finally neuronal degeneration $(32,33)$. Identically, in the liver autophagydefects cause protein accumulation, hepatocyte cell death and liver failure (34). These findings support the pro-survival role for autophagy by maintaining energy homeostasis and removal of toxic products and damaged organelles (35).

Autophagy is continually active at a basal level, however during stress, like hypoxia or nutrient deprivation, autophagy is induced. Previously we showed that ATF4 (transcription factor downstream of the PERK) activates transcription of the autophagy-associated proteins, including microtubule-associated protein light chain 3 beta (LC3B) and autophagy related-5 (ATG5) (36) and GABARAPL1 (this thesis, chapter $\mathbf{3}$ ). In line, in tumours autophagy is mainly localized in hypoxic regions of tumours, sustaining the presence of hypoxic tumour regions (37-40), a feature associated with tumour progression (41).

During autophagy, cytoplasmic content is recognized by substrate specific adaptor proteins that recruit LC3B to the site where the initial membrane, or phagophore, will be generated. LC3B is generated as a precursor protein which is immediately cleaved by autophagy related 4 cysteine peptidase (ATG4) to expose a C-terminal glycine for further modification. This cytosolic form of LC3B is also referred to as LC3B-I. LC3B-I is then conjugated to phosphatidylethanolamine $(\mathrm{PE})$ by an ubiquitination-like conjugation system where ATG7 acts as an ubiquitin-activating enzyme (E1). LC3B-I is transferred to the conjugating enzyme ATG3 (E2) were after it is conjugated to PE, forming the membrane-bound LC3B-II that coats the inner and outer membrane of the doublemembrane, which allows the phagophore to grow. Efficient LC3B-PE formation requires the ATG12-ATG5-ATG16(L) complex. For their interaction, ATG12 is activated by the E1activating enzyme ATG7. Thereafter, ATG12 is transferred to ATG10 (E2-conjugating enzyme) and conjugated to ATG5. ATG5 interacts with ATG16(L) to form the complex, which can form a tetramer via ATG16 homodimerization (reviewed (42)). The complex fulfills multiple functions such as determination of the site for LC3/GABARAP family protein conjugation to $\mathrm{PE}$. The phagophore expands until the content is completely enclosed by the autophagic vesicle, referred to as the autophagosome. Eventually, autophagosomes fuse with hydrolase and protease-containing lysosomes to degrade the content of the autophagosome, which is then effluxed into the cytosol, as amino acids, monosaccharides, fatty acids and nucleotides that are used to maintain biosynthesis and energy homeostasis of the cell.

LC3B turnover is used as a marker of autophagic structures and is used as a "golden standard" in assessing autophagy activation and rate of autophagy (autophagic flux) (43). 
LC3B-II is localized on the intraluminal and cytoplasmic site and autophagosomes. After autophagosome/ lysosome fusion, cytoplasmic LC3B-II is delipidated by ATG4 and recycled. Intraluminal LC3B-II is degraded by after lysosomal hydrolases during autolysosome formation. Therefore, LC3B-II turnover can be used to monitor autophagic activity (44).

Autophagy is considered a doubled-edged sword. In normal cells autophagy has a cytoprotective role by degrading cytotoxic components, and thereby prevents disease. In the tumour microenvironment, autophagy plays a similar role. By activating autophagy, cells survive nutrient depriving conditions, driving hypoxic tumour cells into a more aggressive, therapy resistant and metastatic phenotype. However, the stress tumour cells encounter (hypoxia, nutrient deprivation) are greater, making tumour cells more dependent on autophagy.

\section{The LC3/ GABARAP protein family}

The LC3/GABARAP protein family consists of the LC3 and GABARAP subfamilies and are characterized by high degrees of homology. LC3B is the most studied and characterized member of the protein family and is required for autophagosome formation. The LC3/ GABARAP protein family consisting of microtubule associated protein 1 light chain 3 alpha (LC3A) (2 splice variants), LC3B, microtubule associated protein 1 light chain 3 gamma (LC3C)), and GABA(A) receptor-associated protein (GABARAP), GABA(A) receptor associated protein Like 1 (GABARAPL1) and GABA(A) receptor associated protein Like 2 (GABARAPL2) are implicated in autophagy. The LC3/ GABARAP protein family members share a high degree of homology, though they are unique in function and implicated in autophagy-independent mechanisms as well. An extensive review about autophagy (un-) related functions of all LC3/ GABARAP protein family members is presented in chapter 2 .

Initially, the GABARAP protein family members have been implicated in intracellular trafficking and transport of receptors to the plasma membrane (45-48). For example, GABA (A) receptor associated protein GABARAP derives its name from transport of the GABA(A) receptor (GABAAR) to the plasma membrane. Further research revealed that surface expression of other receptors like the angiotensin II (49), the epidermal growth factor receptor (EGFR) (50) and the human kappa opioid receptor is also mediated by GABARAP family proteins (48). However, retrograde transport is not limited to the transport of receptors alone. The GABARAP protein family, and especially GABARAPL1, is also implicated in (unconventional) secretion and the secretion of extracellular vesicles (chapter $\mathbf{7}$ and $\mathbf{8}$ ). A comprehensive review about autophagy related proteins and secretion is presented in chapter 6 


\section{Extracellular vesicles}

From yeast to mammals, cells secrete extracellular vesicles (EVS) as a vehicle of intercellular communication. EVs are phospholipid bilayer enclosed vesicles that contain bioactive molecules such as nucleic acids (DNA, RNAs, miRNAs), lipids and proteins (receptors, enzymes, transcription factors and extracellular matrix proteins). EV is a generic name for vesicles originating from various cellular compartments including exosomes and microvesicles, but also apoptotic bodies are considered EVs. EVs are classified on their origin: Exosomes, as firstly described by Johnstone et al. (51), are generated during the maturation of early into late endosomes. During this process, intralumal vesicles (ILVs) are formed by inward budding of the endosomal membrane forming multivesicular bodies (MVB) (52). MVBs can either fuse with lysosomes for degradation or fuse with the plasma membrane, resulting in release of the ILVs (exosomes) into the extracellular environment. Microvesicles are a different class of EVs and are released directly by budding from the plasma membrane (53). Although there are no markers to distinguish exosomes and microvesicles after their release, they are all enriched in tetraspanins such as CD9, CD81, and CD63 (53).

In addition to their role in normal physiology, such as their role in T-cell activation (54), EVs are implicated in several steps that stimulate cancer progression and cover most of the hallmarks of cancer described by Hannahan and Weinberg (55-57), including angiogenesis $(18,58)$, increased proliferation(59), increased migration and invasion (60), metastasis (61), immune evasion (62-64), pre-metastatic niche formation $(61,65)$ and inflammation (66).

For example, to meet the increasing demand of oxygen and nutrients of the tumour, blood vessel formation (angiogenesis) is essential. Several studies have shown that EVs contain pro-angiogenic cargo like pro-angiogenic mRNA's (67-69) and growth factors such as vascular endothelial growth factor (VEGF) and platelet derived growth factor (PDGF) (70)(71). GBM tumour cells release pro-angiogenic EVs that are taken up by microvascular endothelial cells in the brain promoting tumour growth and stimulate tumour cell proliferation at distant sites (72). Another study performed with GBM cells, demonstrated that EVs derived from hypoxic GBM cells elicit pro-angiogenic responses in HUVEC cells in vitro and stimulate angiogenesis by inducing cytokine and growth factor secretion and thereby promote pericyte migration (73). Furthermore, EVs are shown to contribute to formation of the pre-metastatic niche $(65,74)$. During the metastatic cascade, tumour cells detach from the primary tumour and disseminate throughout the body. Via the circulatory system circulating tumour cells invade the distant tissue which provides a suitable microenvironment for colonization. Studies have shown that suitable microenvironments can be formed before the arrival of circulating tumour cells (75). EVs contribute to the formation of these predetermined regions, also referred to as pre-metastatic niches $(75,76)$, for instance by destroying the vascular barrier though induction of vascular leakiness (77), extracellular matrix remodelling (78) or 
reprogram bone marrow progenitors cells towards a pro-vasculogenic phenotype (79). Taken together, EVs are involved in a wide variety of cancer associated processes contributing to tumour growth.

\section{Scope of this thesis}

The scope of this thesis is to gain insight in autophagy-associated proteins and their contribution to tumour development with particular focus on GABARAPL1, a member of the LC3/ GABARAP protein family; a group of proteins mainly involved in the recycle and degradation mechanism autophagy. The LC3/ GABARAP protein family is characterized by high degree of structural homology, suggesting that LC3/GABARAP family proteins exert similar functions. In chapter $\mathbf{2}$ we aimed to clarify the functional differences of the LC3/GABARAP protein family members in autophagy (un)-related functions based on available literature. In chapter $\mathbf{3}$ we demonstrate that the expression of the LC3/ GABARAP protein family member GABARAPL1 is induced during hypoxia in several cancer cell lines in a PERK-dependent manner. Additionally we show that GABARAPL1 is required for increased membrane expression of the epidermal growth factor receptor (EGFR) during hypoxia. EGFR is often mutated or amplified in cancer and has a significant impact on tumour development. In chapter 4 we elaborate on the autophagy-dependency of EGFRvIII+ cells during stress conditions and the effect of chloroquine treatment on patients with EGFRvIII expressing tumours. In chapter $\mathbf{5}$ we describe our findings that ATG12 expression is lost in a subset of HNSSC patients. Further we investigated the effects of the lost ATG12 expression on tumour growth, therapy efficacy and tumour control

Autophagy is not exclusively required for the degradation of cellular content, but also mediates unconventional forms of secretion. In chapter 6 we discuss the current knowledge of the physiological roles of autophagy-dependent secretion and the effects on the tumour microenvironment. Also autophagy-dependent receptor trafficking and its involvement in tumour development will be discussed. Further research on GABARAPL1 revealed that GABARAPL1 is required for the release of a subset of proangiogenic extracellular vesicles released during hypoxia (chapter 7). This subset is marked by GABARAPL1 expression on the surface of the vesicle, what makes them accessible for targeting or detection purposes. In this regard, GABARAPL1 ${ }^{+}$EVs are detectable and elevated in the blood of cancer patients. Furthermore, in vitro tests demonstrated that GABARAPL1 ${ }^{+}$EVs are targetable by antibodies, opening the window for biomarker or therapeutic development. EVs can contribute to metastasis formation by preparing the pre-metastatic niche. Therefore we explored the role of GABARAPL1 ${ }^{+}$EV in metastasis formation (chapter 8). Taken together, we show that autophagy is implicated in key cellular mechanisms associated with cell survival and communication in the tumour micro-environment. 


\section{References}

1. WHO. $2018<$ <ttp://www.euro.who.int/en/health-topics/noncommunicable-diseases/cancer/data-andstatistics>.

2. Magagnin MG, Koritzinsky M, Wouters BG. Patterns of tumor oxygenation and their influence on the cellular hypoxic response and hypoxia-directed therapies. Drug Resist Updat 2006;9:185-97

3. Rey S, Schito L, Wouters BG, Eliasof S, Kerbel RS. Targeting Hypoxia-Inducible Factors for Antiangiogenic Cancer Therapy. Trends Cancer 2017;3:529-41

4. Vaupel P, Briest S, Hockel M. Hypoxia in breast cancer: pathogenesis, characterization and biological/therapeutic implications. Wien Med Wochenschr 2002;152:334-42

5. Nordsmark M, Loncaster J, Aquino-Parsons C, Chou SC, Ladekarl M, Havsteen H, et al. Measurements of hypoxia using pimonidazole and polarographic oxygen-sensitive electrodes in human cervix carcinomas. Radiother Oncol 2003;67:35-44

6. Becker A, Hansgen G, Richter C, Dunst J. [Oxygenation status of squamous cell carcinoma of the head and neck: comparison of primary tumors, their neck node metastases and normal tissue]. Strahlenther Onkol 1998;174:484-6

7. Movsas B, Chapman JD, Hanlon AL, Horwitz EM, Pinover WH, Greenberg RE, et al. Hypoxia in human prostate carcinoma: an Eppendorf PO2 study. Am J Clin Oncol 2001;24:458-61

8. Graeber TG, Osmanian C, Jacks T, Housman DE, Koch CJ, Lowe SW, et al. Hypoxia-mediated selection of cells with diminished apoptotic potential in solid tumours. Nature 1996;379:88-91

9. Wouters BG, van den Beucken T, Magagnin MG, Lambin P, Koumenis C. Targeting hypoxia tolerance in cancer. Drug Resist Updat 2004;7:25-40

10. Hockel M, Schlenger K, Aral B, Mitze M, Schaffer U, Vaupel P. Association between tumor hypoxia and malignant progression in advanced cancer of the uterine cervix. Cancer Res 1996;56:4509-15

11. Nordsmark M, Bentzen SM, Rudat V, Brizel D, Lartigau E, Stadler P, et al. Prognostic value of tumor oxygenation in 397 head and neck tumors after primary radiation therapy. An international multi-center study. Radiother Oncol 2005;77:18-24

12. Brizel DM, Scully SP, Harrelson JM, Layfield LJ, Bean JM, Prosnitz LR, et al. Tumor oxygenation predicts for the likelihood of distant metastases in human soft tissue sarcoma. Cancer Res 1996;56:941-3

13. Gort EH, Groot AJ, van der Wall E, van Diest PJ, Vooijs MA. Hypoxic regulation of metastasis via hypoxiainducible factors. Curr Mol Med 2008;8:60-7

14. Gort EH, van Haaften G, Verlaan I, Groot AJ, Plasterk RH, Shvarts A, et al. The TWIST1 oncogene is a direct target of hypoxia-inducible factor-2alpha. Oncogene 2008;27:1501-10

15. Theys J, Jutten B, Habets R, Paesmans K, Groot AJ, Lambin P, et al. E-Cadherin loss associated with EMT promotes radioresistance in human tumor cells. Radiother Oncol 2011;99:392-7

16. Yang MH, Wu KJ. TWIST activation by hypoxia inducible factor-1 (HIF-1): implications in metastasis and development. Cell Cycle 2008;7:2090-6

17. Filipazzi P, Burdek M, Villa A, Rivoltini L, Huber V. Recent advances on the role of tumor exosomes in immunosuppression and disease progression. Semin Cancer Biol 2012;22:342-9

18. Grange C, Tapparo M, Collino F, Vitillo L, Damasco C, Deregibus MC, et al. Microvesicles released from human renal cancer stem cells stimulate angiogenesis and formation of lung premetastatic niche. Cancer Res 2011;71:5346-56

19. Jung T, Castellana D, Klingbeil P, Cuesta Hernandez I, Vitacolonna M, Orlicky DJ, et al. CD44v6 dependence of premetastatic niche preparation by exosomes. Neoplasia 2009;11:1093-105

20. Peinado $H$, Lavotshkin S, Lyden D. The secreted factors responsible for pre-metastatic niche formation: old sayings and new thoughts. Semin Cancer Biol 2011;21:139-46

21. Salomon C, Ryan J, Sobrevia L, Kobayashi M, Ashman K, Mitchell M, et al. Exosomal signaling during hypoxia mediates microvascular endothelial cell migration and vasculogenesis. PLoS One 2013;8:e68451

22. Sceneay J, Parker BS, Smyth MJ, Moller A. Hypoxia-driven immunosuppression contributes to the premetastatic niche. Oncoimmunology 2013;2:e22355 
23. Sceneay J, Smyth MJ, Moller A. The pre-metastatic niche: finding common ground. Cancer Metastasis Rev 2013

24. Svensson KJ, Kucharzewska P, Christianson HC, Skold S, Lofstedt T, Johansson MC, et al. Hypoxia triggers a proangiogenic pathway involving cancer cell microvesicles and PAR-2-mediated heparin-binding EGF signaling in endothelial cells. Proc Natl Acad Sci U S A 2011;108:13147-52

25. Wong CC, Gilkes DM, Zhang $\mathrm{H}$, Chen J, Wei $\mathrm{H}$, Chaturvedi $\mathrm{P}$, et al. Hypoxia-inducible factor 1 is a master regulator of breast cancer metastatic niche formation. Proc Natl Acad Sci U S A 2011;108:16369-74

26. Overgaard J. Hypoxic modification of radiotherapy in squamous cell carcinoma of the head and neck - A systematic review and meta-analysis. Radiother Oncol 2011

27. Nordsmark M, Overgaard M, Overgaard J. Pretreatment oxygenation predicts radiation response in advanced squamous cell carcinoma of the head and neck. Radiother Oncol 1996;41:31-9

28. Nordsmark M, Bentzen SM, Rudat V, Brizel D, Lartigau E, Stadler P, et al. Prognostic value of tumor oxygenation in 397 head and neck tumors after primary radiation therapy. An international multi-center study. Radiother Oncol 2005;77:18-24

29. Chen C, Lou T. Hypoxia inducible factors in hepatocellular carcinoma. Oncotarget 2017;8:46691-703

30. Koritzinsky M, Levitin F, van den Beucken T, Rumantir RA, Harding NJ, Chu KC, et al. Two phases of disulfide bond formation have differing requirements for oxygen. J Cell Biol 2013;203:615-27

31. Kuma A, Hatano M, Matsui M, Yamamoto A, Nakaya H, Yoshimori T, et al. The role of autophagy during the early neonatal starvation period. Nature 2004;432:1032-6

32. Hara T, Nakamura K, Matsui M, Yamamoto A, Nakahara Y, Suzuki-Migishima R, et al. Suppression of basal autophagy in neural cells causes neurodegenerative disease in mice. Nature 2006;441:885-9

33. Komatsu M, Waguri S, Chiba T, Murata S, Iwata J, Tanida I, et al. Loss of autophagy in the central nervous system causes neurodegeneration in mice. Nature 2006;441:880-4

34. Komatsu M, Waguri S, Ueno T, Iwata J, Murata S, Tanida I, et al. Impairment of starvation-induced and constitutive autophagy in Atg7-deficient mice. J Cell Biol 2005;169:425-34

35. Levine B, Kroemer G. Autophagy in the pathogenesis of disease. Cell 2008;132:27-42

36. Rouschop KM, van den Beucken T, Dubois L, Niessen H, Bussink J, Savelkouls K, et al. The unfolded protein response protects human tumor cells during hypoxia through regulation of the autophagy genes MAP1LC3B and ATG5. J Clin Invest 2010;120:127-41

37. Degenhardt K, Mathew R, Beaudoin B, Bray K, Anderson D, Chen G, et al. Autophagy promotes tumor cell survival and restricts necrosis, inflammation, and tumorigenesis. Cancer Cell 2006;10:51-64

38. Karantza-Wadsworth V, Patel S, Kravchuk O, Chen G, Mathew R, Jin S, et al. Autophagy mitigates metabolic stress and genome damage in mammary tumorigenesis. Genes Dev 2007;21:1621-35

39. Mathew R, Kongara S, Beaudoin B, Karp CM, Bray K, Degenhardt K, et al. Autophagy suppresses tumor progression by limiting chromosomal instability. Genes Dev 2007;21:1367-81

40. Rouschop KM, van den Beucken T, Dubois L, Niessen H, Bussink J, Savelkouls K, et al. The unfolded protein response protects human tumor cells during hypoxia through regulation of the autophagy genes MAP1LC3B and ATG5. J Clin Invest 2010;120:127-41

41. Harris AL. Hypoxia--a key regulatory factor in tumour growth. Nat Rev Cancer 2002;2:38-47

42. Geng J, Klionsky DJ. The Atg8 and Atg12 ubiquitin-like conjugation systems in macroautophagy. 'Protein modifications: beyond the usual suspects' review series. EMBO Rep 2008;9:859-64

43. Klionsky DJ, Abeliovich H, Agostinis P, Agrawal DK, Aliev G, Askew DS, et al. Guidelines for the use and interpretation of assays for monitoring autophagy in higher eukaryotes. Autophagy 2008;4:151-75

44. Tanida I, Minematsu-Ikeguchi N, Ueno T, Kominami E. Lysosomal turnover, but not a cellular level, of endogenous LC3 is a marker for autophagy. Autophagy 2005;1:84-91

45. Chen L, Wang H, Vicini S, Olsen RW. The gamma-aminobutyric acid type A (GABAA) receptor-associated protein (GABARAP) promotes GABAA receptor clustering and modulates the channel kinetics. Proc Natl Acad Sci U S A 2000;97:11557-62

46. Kittler JT, Rostaing P, Schiavo G, Fritschy JM, Olsen R, Triller A, et al. The subcellular distribution of GABARAP and its ability to interact with NSF suggest a role for this protein in the intracellular transport of GABA(A) receptors. Molecular and cellular neurosciences 2001;18:13-25 
47. Cook JL, Re RN, deHaro DL, Abadie JM, Peters M, Alam J. The trafficking protein GABARAP binds to and enhances plasma membrane expression and function of the angiotensin II type 1 receptor. Circulation research 2008;102:1539-47

48. Chen C, Wang Y, Huang P, Liu-Chen LY. Effects of C-terminal modifications of GEC1 protein and gammaaminobutyric acid type $A(G A B A(A))$ receptor-associated protein (GABARAP), two microtubule-associated proteins, on kappa opioid receptor expression. The Journal of biological chemistry 2011;286:15106-15

49. Alam J, Deharo D, Redding KM, Re RN, Cook JL. C-terminal processing of GABARAP is not required for trafficking of the angiotensin II type $1 A$ receptor. Regulatory peptides 2010;159:78-86

50. Keulers TG, Schaaf MB, Peeters HJ, Savelkouls KG, Vooijs MA, Bussink J, et al. GABARAPL1 is required for increased EGFR membrane expression during hypoxia. Radiotherapy and oncology : journal of the European Society for Therapeutic Radiology and Oncology 2015;116:417-22

51. Johnstone RM, Adam M, Hammond JR, Orr L, Turbide C. Vesicle formation during reticulocyte maturation. Association of plasma membrane activities with released vesicles (exosomes). J Biol Chem 1987;262:9412-20

52. Kowal J, Tkach M, Thery C. Biogenesis and secretion of exosomes. Curr Opin Cell Biol 2014;29:116-25

53. Colombo M, Raposo G, Thery C. Biogenesis, secretion, and intercellular interactions of exosomes and other extracellular vesicles. Annu Rev Cell Dev Biol 2014;30:255-89

54. De Toro J, Herschlik L, Waldner C, Mongini C. Emerging roles of exosomes in normal and pathological conditions: new insights for diagnosis and therapeutic applications. Front Immunol 2015;6:203

55. Hanahan D, Weinberg RA. Hallmarks of cancer: the next generation. Cell 2011;144:646-74

56. Hanahan D, Weinberg RA. The hallmarks of cancer. Cell 2000;100:57-70

57. Kanada $\mathrm{M}$, Bachmann $\mathrm{MH}$, Contag $\mathrm{CH}$. Signaling by Extracellular Vesicles Advances Cancer Hallmarks. Trends Cancer 2016;2:84-94

58. Tadokoro H, Umezu T, Ohyashiki K, Hirano T, Ohyashiki JH. Exosomes derived from hypoxic leukemia cells enhance tube formation in endothelial cells. J Biol Chem 2013;288:34343-51

59. Soldevilla B, Rodriguez M, San Millan C, Garcia V, Fernandez-Perianez R, Gil-Calderon B, et al. Tumorderived exosomes are enriched in DeltaNp73, which promotes oncogenic potential in acceptor cells and correlates with patient survival. Hum Mol Genet 2014;23:467-78

60. Umezu T, Ohyashiki K, Kuroda M, Ohyashiki JH. Leukemia cell to endothelial cell communication via exosomal miRNAs. Oncogene 2013;32:2747-55

61. Hoshino A, Costa-Silva B, Shen TL, Rodrigues G, Hashimoto A, Tesic Mark M, et al. Tumour exosome integrins determine organotropic metastasis. Nature 2015;527:329-35

62. Clayton A, Mitchell JP, Court J, Linnane S, Mason MD, Tabi Z. Human tumor-derived exosomes downmodulate NKG2D expression. J Immunol 2008;180:7249-58

63. Abusamra AJ, Zhong Z, Zheng X, Li M, Ichim TE, Chin JL, et al. Tumor exosomes expressing Fas ligand mediate CD8+ T-cell apoptosis. Blood cells, molecules \& diseases 2005;35:169-73

64. Clayton A, Mitchell JP, Court J, Mason MD, Tabi Z. Human tumor-derived exosomes selectively impair lymphocyte responses to interleukin-2. Cancer Res 2007;67:7458-66

65. Costa-Silva B, Aiello NM, Ocean AJ, Singh S, Zhang H, Thakur BK, et al. Pancreatic cancer exosomes initiate pre-metastatic niche formation in the liver. Nat Cell Biol 2015;17:816-26

66. Buzas El, Gyorgy B, Nagy G, Falus A, Gay S. Emerging role of extracellular vesicles in inflammatory diseases. Nat Rev Rheumatol 2014;10:356-64

67. Zernecke A, Bidzhekov K, Noels H, Shagdarsuren E, Gan L, Denecke B, et al. Delivery of microRNA-126 by apoptotic bodies induces CXCL12-dependent vascular protection. Sci Signal 2009;2:ra81

68. van Balkom BW, de Jong OG, Smits M, Brummelman J, den Ouden K, de Bree PM, et al. Endothelial cells require miR-214 to secrete exosomes that suppress senescence and induce angiogenesis in human and mouse endothelial cells. Blood 2013;121:3997-4006, S1-15

69. Kang T, Jones TM, Naddell C, Bacanamwo M, Calvert JW, Thompson WE, et al. Adipose-Derived Stem Cells Induce Angiogenesis via Microvesicle Transport of miRNA-31. Stem Cells Transl Med 2016;5:440-50

70. Brill A, Dashevsky O, Rivo J, Gozal Y, Varon D. Platelet-derived microparticles induce angiogenesis and stimulate post-ischemic revascularization. Cardiovasc Res 2005;67:30-8 
71. Wendler F, Favicchio R, Simon T, Alifrangis C, Stebbing J, Giamas G. Extracellular vesicles swarm the cancer microenvironment: from tumor-stroma communication to drug intervention. Oncogene 2017;36:877-84

72. Skog J, Wurdinger T, van Rijn S, Meijer DH, Gainche L, Sena-Esteves M, et al. Glioblastoma microvesicles transport RNA and proteins that promote tumour growth and provide diagnostic biomarkers. Nat Cell Biol 2008;10:1470-6

73. Kucharzewska P, Christianson HC, Welch JE, Svensson KJ, Fredlund E, Ringner M, et al. Exosomes reflect the hypoxic status of glioma cells and mediate hypoxia-dependent activation of vascular cells during tumor development. Proc Natl Acad Sci U S A 2013;110:7312-7

74. Alderton GK. Metastasis. Exosomes drive premetastatic niche formation. Nat Rev Cancer 2012;12:447

75. Kaplan RN, Riba RD, Zacharoulis S, Bramley AH, Vincent L, Costa C, et al. VEGFR1-positive haematopoietic bone marrow progenitors initiate the pre-metastatic niche. Nature 2005;438:820-7

76. Psaila B, Lyden D. The metastatic niche: adapting the foreign soil. Nat Rev Cancer 2009;9:285-93

77. Zhou W, Fong MY, Min Y, Somlo G, Liu L, Palomares MR, et al. Cancer-secreted miR-105 destroys vascular endothelial barriers to promote metastasis. Cancer Cell 2014;25:501-15

78. Shimoda M, Khokha R. Metalloproteinases in extracellular vesicles. Biochim Biophys Acta 2017;1864:1989-2000

79. Peinado H, Aleckovic M, Lavotshkin S, Matei I, Costa-Silva B, Moreno-Bueno G, et al. Melanoma exosomes educate bone marrow progenitor cells toward a pro-metastatic phenotype through MET. Nat Med 2012;18:883-91 



\section{Chapter}

\section{LC3/GABARAP family proteins: Autophagy (un)related functions}

Marco BE Schaaf*, Tom G Keulers*, Marc A Vooijs, Kasper MA Rouschop

Department of Radiation Oncology (Maastro Lab), GROW School for Oncology \& Developmental Biology, Maastricht University Medical Center+, Maastricht, The Netherlands.

* Authors contributed equally Published in FASEB J. 2016 Dec;30(12):3961-3978. 


\section{Abstract}

From yeast to mammals, autophagy is an important mechanism to sustain cellular homeostasis through facilitating the degradation and recycling of aged and cytotoxic components. During autophagy, cargo is captured in double membraned vesicles, the autophagosomes, and degraded though lysosomal fusion. In yeast, autophagy initiation, cargo recognition, cargo engulfment and vesicle closure is Atg8 dependent. In higher eukaryotes, Atg8 has evolved into the LC3/GABARAP protein family consisting of 7 family proteins (LC3A (two splice variants), LC3B, LC3C, GABARAP, GABARAPL1 and GABARAPL2). LC3B, the most studied family protein, is associated with autophagosome development and maturation, and is used to monitor autophagic activity. Given the high homology, the other LC3/GABARAP family proteins are often presumed to fulfill similar functions. Nevertheless, substantial evidence shows that the LC3/GABARAP family proteins are unique in function and important in autophagy-independent mechanisms.

In this review we discuss the current knowledge and function(s) of the LC3/GABARAP family proteins. We focus on processing of the individual family proteins end their role in autophagy initiation, cargo recognition, vesicle closure and trafficking, a complex and tightly regulated process that requires selective presentation and recruitment of these family proteins. In addition, autophagy unrelated functions of the LC3/GABARAP protein family members are discussed.

Keywords: autophagy, cancer, ATG7, Atg8 orthologues, LC3/GABARAP family proteins 


\section{Introduction}

Autophagy (from the Greek auto-, "self" and phagein, "to eat") is a catabolic process required to maintain cellular homeostasis through directing old/damaged organelles (e.g. mitochondria), protein aggregates and pathogens for lysosomal degradation. The products of lysosomal breakdown (e.g. amino acids, fatty acids) are recycled and transported across the lysosomal membrane into the cytoplasm. These metabolites can be re-used in protein and ATP production (reviewed in (1)). During metabolic stress or periods of insufficient nutrient supply, autophagy is rapidly upregulated to sustain energy production and provide building blocks for essential cellular functions. In line, cells deprived of glucose or amino acids, or exposed to hypoxia depend on autophagy for their survival $(2,3)$. In short, autophagy acts as a pro-survival mechanism through maintaining energy homeostasis and removal of toxic protein aggregates, pathogens and old/damaged organelles (4). Hence, defective autophagy in brain causes accumulation of damaged mitochondria and protein aggregates leading to neuronal degeneration (5). Similarly, autophagy-defects result in protein accumulation in the liver, causing hepatomegaly, hepatocyte cell death and liver failure (6).

Initiation of autophagy commences with the formation of an initial membrane structure, the phagophore (nucleation). As the phagophore elongates, it (non-)selectively sequesters cargo and closes into a double-membrane vesicle, the autophagosome. The autophagosome then fuses with a lysosome and exposes cargo to the lysosomal enzymes causing degradation of the content, a process well-conserved from yeast to humans. Research in yeast has provided insights into the role of essential proteins in autophagy. This has led to the identification of at least 38 AuTophaGy-related proteins (Atg), of which many are functionally conserved in eukaryotes. In yeast, Atg8 is a limiting protein in expansion of the phagophore (7). Atg8 levels correspond with autophagosome size, as reduced Atg8 leads to the development of smaller autophagosomes with less favorable volume-to-surface ratios and attenuation of autophagy (7). In addition, Atg8 participates in cargo recruitment to the autophagosome by interacting with cargo binding proteins (e.g. Atg19) (8). For activation, Atg8 requires the covalent conjugation to phosphatidylethanolamine (PE) that enables its anchoring into the autophagosomal membranes, a process tightly orchestrated by Atg4, Atg7,Atg3 and the Atg12-Atg5Atg16L1 complex (9).

In mammals, at least seven Atg8 orthologues exist; microtubule associated protein 1 light chain 3 alpha MAP1LC3A (two splicing variants hereafter referred as LC3A-a and LC3A-b), -beta (MAP1LC3B (LC3B), -gamma (MAP1LC3C (LC3C), GABA type A receptorassociated protein (GABARAP), GABARAP Like 1 (GABARAPL1/gec1) and GABARAP Like 2 (GABARAPL2/GATE16). The most studied and best understood LC3/GABARAP family protein is LC3B. LC3B is essential for the execution of autophagy and therefore the widely accepted marker for autophagy activity assessment. The LC3/GABARAP family proteins are produced from separate transcripts, derived from their own genomic loca- 
tion (table 1). Nevertheless, they share a high sequence similarity and have a conserved carboxy-terminal glycine. In comparison to LC3B, LC3A-a, LC3A-b, LC3C, GABARAP, GABARAPL1 and GABARAPL2 display 92\%, 94\%, 71\%, 59\%, 60\% and 65\% similarity, respectively. Each LC3/GABARAP family protein is characterized by two amino-terminal $\alpha$-helices and a carboxy-terminal ubiquitin core (10). LC3/GABARAP family proteins are often presumed to fulfil similar functions as LC3B. Functional differences and autophagy independent functions are frequently neglected and may lead to incomplete interpretation of data. Within this review we have provided an overview of the current knowledge on the function of the LC3/GABARAP family proteins.

We have attempted to provide information on several questions raised: e.g. a) Are their functions limited to autophagy? b) Are they all crucial for the execution of autophagy? c) What is their role in this process? d) Do they contribute to recruitment of selective targets for autophagy? If so, how is selectivity and recruitment organized? e) Is there functional redundancy? And f) how are LC3/GABARAP family proteins selectively recruited to the required site? In this manuscript, we address these questions by assembling the current knowledge on processing, regulation, function and specificity of the LC3/GABARAP family proteins. By connecting the data described in literature, we highlight the underlying complexity in the mammalian autophagy machinery to permit a more complete interpretation of new experimental results in the future.

Together, the provided data indicates that not all LC3/GABARAP family proteins are redundant in the autophagy process and each has a unique (autophagy independent) important function for cell homeostasis.

\section{Functions of LC3/GABARAP family proteins in autophagy (table 1)}

\section{Initiation, elongation and closure of the autophagosome}

Whether the membrane for nucleation is formed de novo or is derived from an organelle is not fully understood yet. Membrane components can be derived from endoplasmic reticulum (ER), Golgi complex, endosomes, plasma membrane and ERmitochondria contact sites and may be driven by the distance of the source to the initiating membrane (reviewed in (11)). Recently, it has become clear that the Unc51-like kinase 1 (ULK1) functions as a facilitator of phagophore formation. ULK1 is present in complexes consisting of ULK1, ATG13, FIP200 and ATG101 and can alter the complex's activity through increased kinase activation (12) and is shown to be crucial during stress conditions (13). ULK1 phosphorylates beclin 1 (BECN1), a member of a second crucial autophagy-related complex and implicated in ER-derived phagophore formation (14). Part of this complex is a phosphoinositol 3 kinase (PI3K; Vps34) which phosphorylates phosphosphatidylinositol (PI) at position 3 to produce PI(3)P. PI(3)P serves as a docking site for effectors to further mediate phagophore maturation and elongation (15). Pull- 
down of ectopically expressed LC3/GABARAP family proteins revealed that GABARAP and GABARAPL1 display highest affinity to ULK1 and LC3B the lowest. This suggests that GABARAP subfamily proteins can, more efficiently than LC3B, serve as scaffolding proteins by recruiting ULK1 and beclin 1 (complex) to the site of nucleation (16) (Fig 1A). Whether this indicates that phagophore development or cargo engulfment is dependent on GABARAP proteins or that GABARAP proteins allow selective cargo engulfment remains unknown.

After elongation autophagosome closure the autophagosomes is formed (Fig. $1 \mathrm{C}$ and D) and requires fusion events of both the inner and outer membrane leaflets (Fig. 1D). In eukaryotic cells, specialized functions seem to be attributed to specific LC3/GABARAP family proteins. For example, cells deficient in the LC3 subfamily (LC3A, LC3B, LC3C) or GABARAP subfamily (GABARAP, GABARAPL1, GABARAPL2) display impaired autophagosome formation (17). Yet, knockdown of the LC3- or GABARAP subfamily proteins leads to different autophagy impairment phenotypes, as deficiency in the LC3 subfamily (LC3A, LC3B and LC3C simultaneously) leads to generation of smaller autophagosomes whereas deficiency of GABARAP subfamily (GABARAP, GABARAPL1 and GABARAPL2 simultaneously) leads to biogenesis of larger autophagosomes. Reexpression of LC3B or GABARAPL2 is sufficient to rescue autophagy. Here, LC3B rescues phagophore elongation and GABARAPL2 mediates closure of the vesicle(17). These results are in line with observations in cell free systems where the amino-terminal $\alpha$ helices of GABARAPL2 and LC3B promote tethering and fusion of membranes. Although the fusion activity of GABARAPL2 and LC3B are similar, the fusion activity of LC3B is mediated by ionic interactions, whereas those of GABARAPL2 are mediated by hydrophobic interactions(18). Interestingly, GABARAP family proteins are present in an open and closed conformation. Herein, the open form has a protruding amino-terminal helix with hydrophobic residues that enables penetration into the adjacent membrane and interacts with the hydrophobic center of the lipid bilayer (19). In contrast, LC3B interacts with the lipid head group (18). Together, these events can bring together (extending) membranes and lead to phagophore elongation (hemifusion, Fig. 1C) and closure (fusion, Fig. 1D). Whether the transition from elongation to closure is merely chance or is an orchestrated event when the cargo is engulfed remains unclear. These data suggest little redundancy between LC3/GABARAP family protein function as functions appear to be inherent to their structural characteristics. 


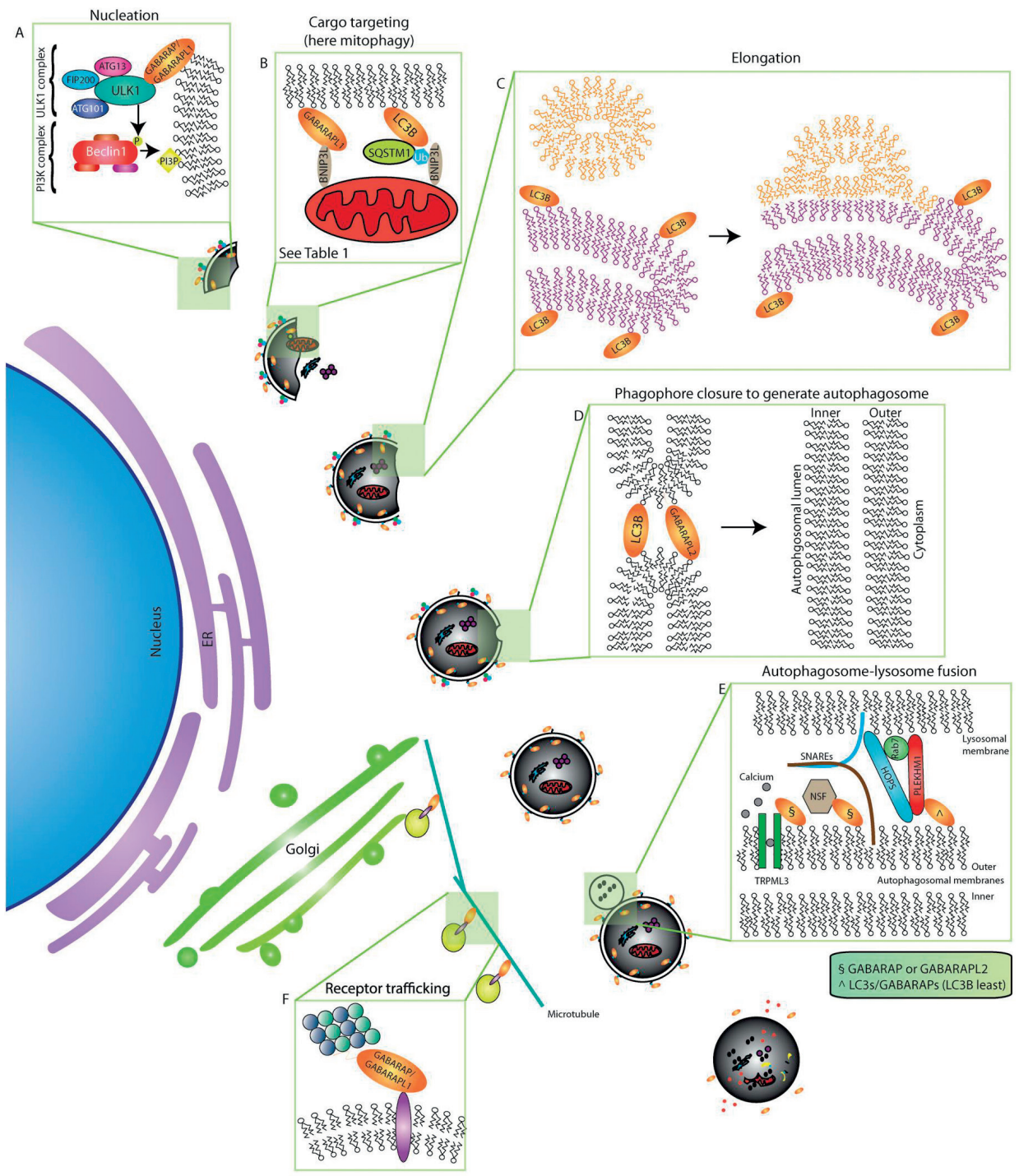

Figure 1. Involvement of LC3/GABARAP family proteins in (non-)autophagy related processes.

Autophagy initiation involves the formation of a phagophore. This nucleation phase $(\mathbf{A})$ is commenced by two protein complexes, ULK1 complex and Beclin1 complex. Herein, the first can be localized to the phagophore by binding predominantly GABARAP/GABARAPL1. The ULK1 complex activation status influences the rate of autophagy by Beclin1 phosphorylation. The phagophore can engulf content either selectively or nonselectively. Selectivity in cargo tethering to the phagophore (B) is established by the LC3/GABARAP family members that have different intrinsic properties that allows the binding linker proteins. Here mitophagy is illustrated by the protein BNIP3L/Nix at the mitochondrial membrane that can bind GABARAPL1 directly or be ubiquitinylated for SQSTM1 mediated recognition. Elongation (C) requires the delivery of membrane components (orange) to the growing phagophore (purple). LC3B is the main family protein to effectuate elongation. Phagophore closure (D) generates a closed double membraned vesicle. Fusion of both membrane leaflets is 
required for closure that is thought to be done by LC3B and GABARAPL2 in a cooperative fashion. Subsequent fusion of the autophagosome with a lysosome $(\mathbf{E})$ is required to acquire the degradative enzymes and acidification of the autophagosomal lumen for cargo degradation. This fusion event requires GABARAP and GABARAPL2 by their interaction with NSF and the calcium channel TRPML3, which both facilitate fusion. Moreover, all LC3/GABARAP proteins (notably LC3B the least) interact with PLEKHM1 that mediates bridging both vesicles. Besides autophagy, recent findings indicate the relevance of, in particular, GABARAP family proteins in receptor trafficking (F). These are involved as adaptor proteins connecting the receptors in golgiderived vesicles to tubulin fibers (also see table 1 ).

\section{Cargo targeting}

Initially, autophagy was considered a process of non-selective degradation. However, increasing evidence indicates that cargo can selectively be targeted for degradation. Selective autophagy has been described for aggregated proteins (aggrephagy), mitochondria (mitophagy), peroxisomes (pexophagy), ribosomes (ribophagy), endoplasmic reticulum (reticulophagy), lipid droplets (lipophagy) and pathogens (xenophagy); however much remains to be elucidated regarding the regulation and molecular mediators involved. For the engulfment of several specific substrates, LC3/GABARAP family proteins play a pivotal role through interaction with autophagy adaptor proteins such as sequestosome 1 (SQSTM1/p62, hereafter referred to as SOSTM1), neighbor of BRCA1 gene 1 (NBR1), calcium binding and coiled-coil domain 2 (NDP52/ CALCOCO2), BCL2/adenovirus E1B 19kDa interacting protein 3-like (NIX/BNIP3L), optineurin (OPTN), and WD repeat and FYVE domain containing (ALFY) (table 1). These are able to bind both the ubiquitinated substrate/cargo and LC3/GABARAP family proteins through their LC3 interacting regions (LIRs)(20) and facilitate cargo-tethering to the luminal side of the phagophore. The combination of LC3/GABARAP protein and adaptor protein is therefore essential in cargo selection, targeting and degradation.

Aggrephagy: Protein aggregates and peroxisomes are degraded through autophagy. In this SQSTM1 plays an essential role (21). Although SQSTM1 displays no differences in LC3/GABARAP family protein affinity, only lipidated LC3B is able to recruit SQSTM1 to autophagosomes(22). Interestingly, ALFY (an autophagy receptor required for aggrephagy(23)), co-immunoprecipitates predominantly with GABARAP. The binding of ALFY to GABARAP is indispensable for the recruitment of LC3B-positive structures and the degradation of SQSTM1 structures (24). This suggests that GABARAP and LC3B function in a cooperative fashion during aggrephagy to establish a selective form of autophagy. Recently, a new class of ubiquitin-atg8 receptors (CUET proteins) have been identified. The yeast CUE-ubiquitin-binding-domain protein Cue5 and its human homolog toll interacting protein (TOLLIP) mediate the clearance of polyQ protein aggregates. PolyQ proteins contain a polyQ tract, as a result of trinucleotide repeat, cytosine-adenineguanine (CAG) expansions, which encodes glutamine. Their aggregates are linked to neurodegenerative disorders like Huntington's disease. TOLLIP contains two LIR motifs for interaction with LC3B The interaction with LC3 allows spatial organization of TOLLIP 
recruitment and subsequent ubiquitination through CUE recruitment and degradation of the aggregate. Interactions with other members of the LC3/GABARAP proteins family remain matter of investigation (25).

Xenophagy: In the elimination of intracellular pathogens, LC3C is uniquely required due to its strong preferential binding to the autophagy receptor, NDP52/CALCOCO2. Salmonella Typhimurium, damages its surrounding vacuole membrane after invasion, leading to exposure of host glycans and microbial carbohydrates. The 'vesicle damaging receptor' galectin 8 /LGALS8 recruits NDP52 and results in activation of bacterial autophagy(26). The binding of LC3C to NDP52 leads to the engulfment of the bacterium in the autophagosome. After entrapment, NDP52 is required for the maturation and degradation of Salmonella Typhimurium-containing autophagosomes through interaction with, LC3A, LC3B and GABARAPL2 $(27,28)$. The binding of LC3C and the other LC3/GABARAP family proteins is dependent on the presence of two distinct LIRs. Because of the essential role of "LC3C-LIR", its role in xenophagy is non-redundant. In line, Galectin8, LC3C or NDP52 knockdown cells are defective in blocking proliferation of Salmonella (29), indicating a unique cooperation in xenophagy. Additionally, upon phosphorylation by TANK binding kinase 1 (TBK1) to enhance LC3 binding affinity, the autophagy receptor optineurin (OPTN) targets similar cytosolic bacteria as NDP52. OPTN mutants that are deficient in LC3 binding or silenced OPTN results in impaired degradation of ubiquitylated Salmonella enterica (30). In addition to Salmonella, P62 and NDP52 also sequester intracytosolic Shigella and the membrane remnants to the autophagosome for degradation (31,32)

Tax1 binding protein1 (TAX1BP1) is also involved in clearance of intracellular Salmonella and also depends on ubiquitination of the substrate. TAX1BP1 not only binds to the LC3 subfamily members LC3B and LC3C but also interacts with GABARAPL1 and GABARAPL2. In contrast to NDP52 and OPTN, there is no preference in binding affinity for LC3B of LC3C although it shares the same canonical LIR motif as OPTN and NDP52 (33).

Furthermore, the TRIM family plays a role in antiviral defense by targeting viral capsid proteins. Mandell et al found that the TRIM family, and especially tripartite motif containing5 (TRIM5 $\alpha$ ), interacts preferably with GABARAP and GABARAPL1, and to a lesser extend LC3A, LC3C and GABARAPL2. Although GST-pulldown assays show only a weak interaction with LC3B, co-immunoprecipitation studies and immunostainings show that LC3 B may nonetheless be in complexes with TRIM5 $\alpha$. TRIM5 $\alpha$ contains two LIR motifs. Mutation of these motifs results prevented the HIV capsid protein p24 from degradation (34).

Glycophagy: Several myopathies are associated with defects in autophagic and lysosomal degradation of glycogen, illustrating its importance in maintaining glycogen homeostasis (35). In normal functioning, glycogen is hydrolyzed to glucose by lysosomal $\alpha$ glycosidase and is therefore an important source of energy. Starch-binding domain containing protein 1 (STBD1/genethonin 1) binds glycogen and anchors it to mem- 
branes, thereby affecting its cellular localization and its intracellular trafficking to lysosomes (36). STBD1 interacts with GABARAPL1 and to lesser extent GABARAP (37). Mutations and deletions in STBD1's motif to bind GABARAP subfamily proteins indicate that this motif is required for this GABARAPL1-mediated engulfment of glycogen into vesicles (38). Although the interaction has been well established, the exact role of GABARAPL1 in glycophagy remains to be elucidated in functional assays.

Mitophagy: Mitochondrial metabolism leads to the formation of by-products that lead to oxidative stress, DNA damage and mutations and increases in aged and dysfunctional mitochondria. Sustaining a healthy population of mitochondria is therefore critical to maintain cellular homeostasis and prevent accumulation of damaging agents. The turnover of mitochondria is autophagy-dependent and inhibition leads to accumulation of mitochondria and excessive exposure to reactive oxygen species (ROS) (39).Like other substrates, mitochondria can be ubiquitylated for degradation. Interestingly, there is some overlap in receptors involved in the recognition of other ubiquitylated substrates e.g. in xenophagy. After depolarization of the mitochondrial membrane, PINK accumulates in the outer mitochondrial membrane and recruits NDP52 and OPTN, but not p62, to activate mitophagy independently of PARKIN. Interestingly, in knockout models of the 5 mitophagy receptors (TAX1BP1, optineurin, NBR1, p62 and NDP52), Lazarou et al found that LC3C is recruited by TAXBP1 during mitophagy and LC3B by OPTN and NDP52. Furthermore they observed that GABARAP family proteins are not recruited to mitochondria, suggesting that they are not actively involved in mitophagy (40).

In contrast, Novak et al found that GABARAPL1 colocalizes with depolarized mitochondria during basal, starvation and apoptotic conditions (41-43). GABARAPL1 deficiency attenuates autophagic flux and accumulates SQSTM1 including mitophagy and results in accumulation of damaged mitochondria (43). Whether GABARAPL1 is directy involved in mitophagy or influences mitophagy through modulation general autophagy activity remains matter of investigation. Nevertheless, the direct colocalization of GABARAPL1 with mitochondria and its interaction with another important factor in mitophagy, BNIP3L/Nix suggests a direct role for GABARAPL1 in mitophagy (41) (Fig. 1B).

FUNDC1, an integral mitochondrial outer-membrane protein, acts as a specific receptor for mitophagy in an ubiquitin independent manner. In response to hypoxia FUNDC1 is able to bind LC3 proteins -A and -B and GABARAP and GABARAPL2 via its typical LIR domain ( $(Y x x L)$. Upon hypoxia stimulation, Scr kinase is inactivated and the FUNDC1 LIR domain is dephosphorylated, resulting in an increased colocalization and interaction FUNDC1 and LC3B (44). Nix and its homolog Bnip3 also play a role in hypoxia induced mitophagy. Bnip3 and Nix, both members of the BCL2 family, are anchored in the outer mitochondrial membrane via their $\mathrm{C}$ terminal transmembrane domains, BNip3 and Nix contain an identical LIR motif (WxxL). Phosphorylation of the ser17 and ser24 specifically promotes LC3B and GABARAPL2 binding to Bnip3(45). 
Ubiquitin-dependent cargo recognition can be executed by multiple different receptors. Intriguingly, there seems to be an underlying layer of complexity when it comes to LC3/GABARAP proteins family member recruitment. For instance, during the clearance of cytosolic salmonella, TAX1BP1 recruits members of the LC3 and GABARAP protein subfamily. However, during mitophagy, TAX1BP1 selectively recruits LC3C and no GABARAP proteins. It remains matter of investigation of how selective autophagy receptors recruit specific LC3/GABARAP proteins family members to targeted cargos in an ubiquitin (in-) dependent manner.

\section{Autophagosome-lysosome fusion}

The autophagosome can fuse with a lysosome directly to form the autolysosome or fuse with multiple components of the endocytic pathway including early and late endosomes or multivesicular bodies (MVBs) to generate amphisomes(46). Fusion of autophagosomes or amphisomes with acidic lysosomes is required for content degradation. RAB7, a member of a family of small GTPases, is important in lysosome fusion (47) through interaction with homotypic fusion and protein sorting (HOPS) complex. HOPS functions as a guanine nucleotide exchange factor (GEF) to facilitate RAB7 activation and is a downstream effector of RAB7 to promote membrane tethering. For this, HOPS interacts with SNAP (soluble NSF (N-ethylmaleimide-sensitive factor)) attachment protein) receptors (SNAREs) that can interact with other SNAREs through coiled-coil type interactions to facilitate membrane fusion. Full fusion of the autophagosome with the vacuole requires NSF/SEC18 (48), which changes the SNARE'S conformation and allows binding to its interactor (49). Interestingly, GABARAPL2 and GABARAP are both able to interact with NSF $(50,51)$. In addition, GABARAPL2 interacts with transient receptor potential cation channel, mucolipin subfamily, member 3 (TRPML3) (52). This is a $\mathrm{Ca}^{2+}$ permeable cation channel that is proposed to facilitate fusion events by promoting interbilayer contact (53). Thus, GABARAPL2 (and GABARAP) facilitate fusion by scaffolding NSF and TRPML3 at the location of fusion (Fig. 1E).

In addition, pleckstrin homology domain-containing family M member 1 (PLEKHM1), a RAB7 effector which also binds components of the HOPS complex can function as a bridge between autophagosomes and lysosomal membranes. Pulldown analysis demonstrated that PLEKHM1 interacts with all LC3/GABARAP family proteins. Notably, the yield of LC3B was lowest (54) suggesting that autophagosome-lysosome fusion is more dependent on other LC3/GABARAP family proteins.

In this section the different involvement and functions of the LC3/GABARAP family proteins in phagophore formation, elongation and closure, autophagosome-lysosome fusion and selective forms of autophagy have been highlighted. Although structurally these family proteins share high homology, each one seems to fulfill (a) unique function(s) in the execution of autophagy. 


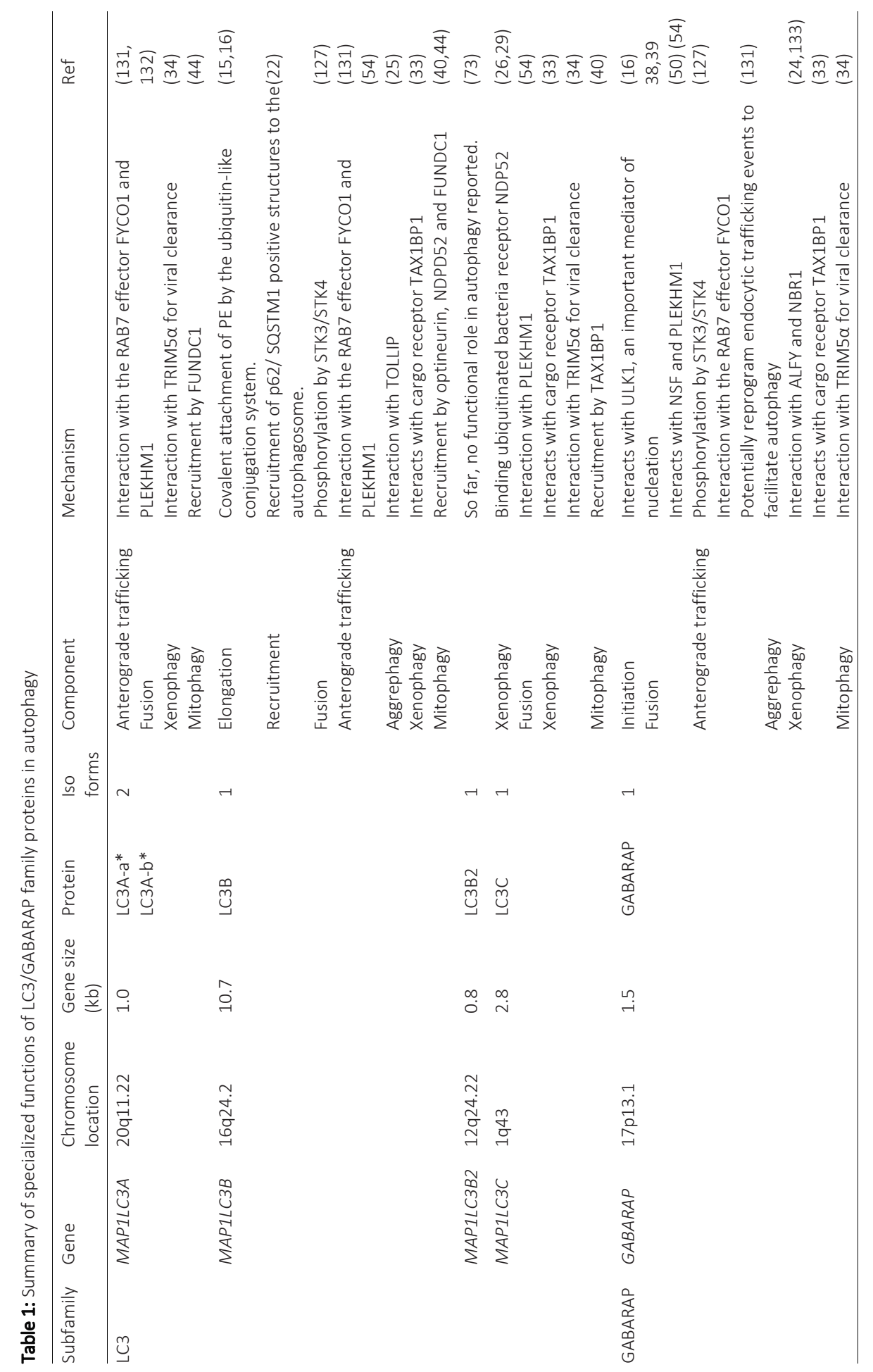


Chapter 2

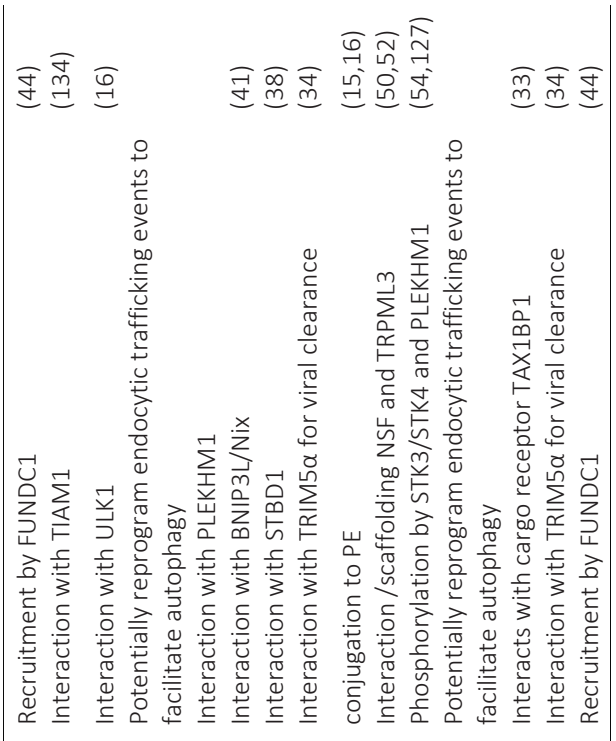

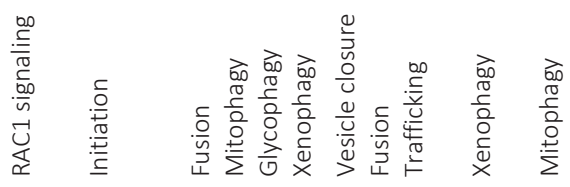

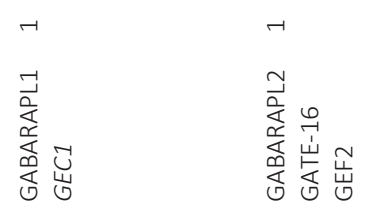

$\stackrel{\circ}{\circ}$

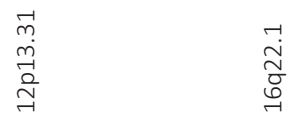

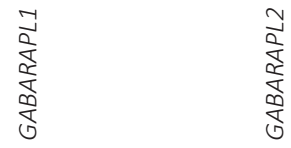

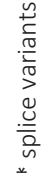




\section{Functions of LC3/GABARAP family proteins independent of autophagy?}

Although all LC3/GABARAP family proteins seem to fulfill (specific) roles within the execution of autophagy, some of the family proteins also fulfill functions that appear canonical autophagy-independent. Several LC3/GABARAP family proteins have been implicated in receptor-trafficking, while others have been implicated in tumour-suppressive functions that appear autophagy independent.

\section{Intracellular trafficking}

Initially, the GABARAP protein family was described in intracellular transport of the GABA (A) receptor (GABAAR)(55). Further studies revealed that GABARAP also mediates membrane expression of the angiotensin II receptors (AGTR) $(56,57)$, the human kappa opioid receptor (hKOPR) (58) and the transient receptor potential cation channel subfamily $\mathrm{V}$ member 1 (TRPV1) (59).

After assembly in the ER, GABAARs are transported to the golgi and are incorporated into transport vesicles (55). To facilitate receptor transport from the golgi to the plasma membrane, GABARAP interacts directly with the $\gamma 2$ subunit of the GABAAR. Here, GABARAP possibly serves as a cargo-receptor required for GABAAR incorporation into the transport vesicle. Interestingly, GABARAP knockout mice show no defect in GABAAR trafficking, indicating that other molecules, such as GABARAPL1 can compensate for the loss (60).

Transport vesicles migrate along microtubules towards their destination. Anterograde transport, trafficking towards the plasma membrane, is facilitated by motor proteins of the kinesin family. Kinesin family member 5A (KIF5A) and kinesin family member 21B (KIF21B) control GABAAR surface expression via interaction with GABARAP $(61,62)$. Yeast two hybrid screens also identified interactions with GABARAPL1 and GABARAPL2, however if these interactions are functional, remain elusive (61). In addition, Monastyrska et al., proposed that vesicles exposing LC3/GABARAP protein family members on their surface, can be 'docked' on the microtubule by their highly positively charged Nterminal region tubulin binding motif until further processing (63). Interestingly, this tubulin binding motif is conserved throughout the whole LC3/GABARAP protein family (64). Furthermore, the interaction of GABARAP with the endocytosis associated protein clathrin suggests that GABARAP may also be involved in the receptor internalization (65). Hence, Lainez et al. showed that internalization and recycling of the TRPV1 is promoted by GABARAP, without altering the degradation rate of the receptor (59).

When transport vesicles reach their destination, membrane fusion is required for further processing. Fusion of transport vesicles with target membranes is mediated by SNARE proteins. $\mathrm{N}$-ethylmaleimide-sensitive factor (NSF) plays a role in the dissociation of SNARE complexes after membrane fusion, allowing them to be recycled for subsequent rounds of fusion. NSF also plays a role in the sorting of receptors into transport 
vesicles and can bind GABARAP and the GABAAR (66). Interestingly, GABARAP is not found at GABAergic sites, but mainly in intracellular vesicles and at the end of golgi stacks where transport vesicles bud and fuse, suggesting its main role is transportation of these vesicles and not anchoring on the plasma membrane $(66,67)$. Although, increasing GABARAP expression leads to enhanced GABAAR expression at the plasma membrane (68), Gephyrin is required for GABAAR clustering (69). Since GABARAP is the only molecule that is able to bind both tubulin, gephyrin and the GABAAR, GABARAP may be the adaptor molecule between gephyrin and the GABAAR (70). Like all members of the LC3/GABARAP family, the C-terminal glycine of GABARAP can be conjugated to PE to establish its incorporation in the membrane. This modification is required for transportation of the GABAAR (58), but is not required for trafficking of the Angiotensin II type A1 receptor (57).

It addition to GABARAP, GABARAPL1 is required for anterograde transport of the kappa opioid receptor (KOR). Knockdown of either GABARAPL1 or GABARAP decreases the surface expression of kappa opioid receptor (58). Recently, we showed that the expression of GABARAPL1 during hypoxia is not restricted to the cytoplasm, but also localizes at the intraluminal side of the plasma membrane of cancer cells. At these sites, GABARAPL1 co-localizes with epidermal growth factor receptor (EGFR). GABARAPL1 knockdown cells display reduced EGFR expression at the plasma membrane, whereas total EGFR levels are not altered (71). Similar results were also observed for carbonic anhydrase IX (CAIX), a hypoxia inducible factor-1 (HIF-1) transcriptional target (71), suggesting that GABARAPL1 plays a role in membrane expression of proteins during hypoxia.

In contrast, GABARAPL2 is a membrane transport enhancer involved in intra-Golgi trafficking (72) (Fig. 1F). The Golgi complex is considered the central component of the secretory pathway required for lipid and protein modification, classification, packaging into specific carriers and delivery. GABARAPL2 stimulates the ATPase activity of NSF and is capable of interacting with GOS-28, a Golgi specific SNARE. The coupling between NSF activity and SNAREs activation allows GABARAPL2 to modulate intra-Golgi transport (51).

As summarized above, the GABARAP family proteins facilitate receptor trafficking, actions that appear independent of autophagy. This illustrates the complexity of data interpretation in (genetic) models that change expression of these factors.

\section{Oncogene/Tumour suppressor}

LC3A is expressed in two isoforms, LC3A-a and -b (NP_115903 and NP_852610). They differ in amino-terminus sequence as a result of a different transcriptional start site. LC3A-a, but not LC3A-b, is turned over by lysosomes and whereas LC3A-a is ubiquitously expressed in most tissues, LC3A-b expression is low in general. Interestingly, LC3A-a expression, but not LC3B, is silenced in $45.5 \%$ of human cancer cell lines originating 
from various tissues. Methylation of the CpG island proximal to the LC3A-a transcriptional start site is observed in human cancer cell lines tested by COBRA (combined bisulfite restriction analysis), while no aberrant methylation was observed in LC3A-a expressing lines. Interestingly, LC3A-a re-expression in LC3A-a silenced cells decreases xenograft growth, suggesting a role for LC3A-a in tumour progression (73).

VHL (Von hippel Lindau) syndrome is an autosomal dominant disease cancer syndrome associated with loss of E3 ligase/tumour suppressor gene VHL which increases predisposition to many tumours including renal cancer. In the majority of spontaneous clear cell renal cell carcinomas (ccRCC) VHL is deleted (74). In renal tumours without loss of VHL, high levels of LC3C are observed. Knockdown of LC3C in these VHL expressing ccRCC cells increases the incidence of orthotopic tumour formation. Interestingly, in these models tumour progression is associated with increased LC3B-mediated autophagy. While LC3B-mediated autophagy is required for tumour progression, LC3C acts as a tumour suppressor (75). This suggests that LC3C functions here independent of canonical (autophagosomal-lysosomal degradation) and LC3B-mediated autophagy to maintain homeostasis. Interestingly in colorectal cancer high LC3B expression is correlated with improved survival after surgery (76). This suggests that LC3B associated autophagy can promote or repress tumorigenic events dependent on the tumour site and potential other mechanisms at play.

Recently, GABARAP has been indicated as a tumour suppressor gene. Compared to normal breast tissue, primary breast cancer tissues contain lower GABARAP mRNA and protein levels, this is in agreement with results observed in cell lines. Ectopic expression of GABARAP gene in low-expressing breast cancer cell lines reduced tumour growth rate (77). Moreover, tumour incidence induced by mutagenic and immunotoxic 7,12dimethylbenz(a)anthracene (DMBA) treatment was reduced in GABARAP knock out (KO) mice. Interestingly, injection of GABARAP proficient melanoma cells in GABARAP knock out mice led to reduced tumour growth, supporting the tumour suppressive role of GABARAP (78). This tumour suppressive feature of GABARAP could be mediated through direct effects on autophagy and/or in an indirect fashion by controlling receptor trafficking such as EGFR (79) (71)).

\section{ER-associated degradation (ERAD)}

The ER requires a quality control system to ensure correct protein folding. Misfolded proteins are selected for degradation by processes collectively referred to as ERassociated degradation (ERAD). At steady state, the effector proteins in ERAD (including EDEM1 and OS9) are removed rapidly from the ER to establish low ER residence, defined as ERAD tuning. EDEM1 turnover is reduced in mouse embryonic fibroblasts lacking ATG5 or treated with chloroquine. Gradient separated fractions showed that a small fraction of EDEM1 is associated with bulk ER; however, the majority of EDEM1 is present in other fractions, fractions with LC3-I (80). This suggests that LC3 coated vesicles 
that differ from autophagosomes (as LC3-I rather than LC3-II is associated) are required for the disposal of EDEM1 in the process of ERAD tuning.

\section{LC3-associated phagocytosis}

During phagocytosis a cell engulfs particles (as cells or pathogens) to create an intracellular vesicle known as a phagosome. Recently it was observed that these single membrane vesicles can express LC3(81). The process is referred to as LC3-associated phagocytosis, or LAP. LC3 lipidation is required for its association with these phagosomes, and is dependent on an array of autophagy related proteins including ATG5, ATG7, Beclin1, ATG12, and ATG16L (81-83). However, other autophagy related proteins seem to be dispensable for LAP including ULK1/2, ATG13, FIP200 and ATG14(83). In addition, LAP specific proteins (dispensable for autophagy) are Rubicon (a Beclin1 interacting protein) and ROS-producing NADPH oxidase 2 (NOX2) (84). Recently, Martinez et al exploited this segregation in required proteins between autophagy and LAP by inducing LAP specific abrogation in immune cells and challenging mice with apoptotic cell injections. The phenotype that these mice presented included accumulation of lymphocytes, monocytes, activated CD8+ cells, developed autoantibodies and kidney damage with increased interferon gamma expression. This phenotype resembles aspects of systemic lupus erythematosus (SLE) patients and is the result of increased pro-inflammatory cytokine secretion. Therefore, LAP was suggested to be required to protect against certain autoimmune diseases (85). Nevertheless, phagosome maturation can occur independent of LC3(86), thus requirement of LC3 and other ATG proteins for phagosome maturation is possibly condition dependent. Nevertheless, LAP facilitates antigen presentation on major histocompatibility complex (MHC) class II molecules (87).

\section{V. motility and growth}

Ras-related C3 botulinum toxin substrate 1 (RAC1) mediates cell motility and growth. RAC1 activity is controlled by the guanine exchange factor TIAM1. The recently discovered CUL3 KBTBD6/KBTBD7 ubiquitin ligase complex controls TIAM1 abundance by ubiquitination and targeting for proteasomal degradation. Interestingly, ubiquitination of TIAM1 depends on binding to GABARAP, located on GABARAP positive vesicles, resulting in restriction of RAC1 signaling to specific sites in the cell. For binding to GABARAP, KBTBD6/7 contains a LIR motif to bind preferably to GABARAP proteins rather than the LC3 subfamily. Furthermore, the authors show that these GABARAP positive vesicles are not associated with autophagosome formation or degradation, suggesting a vesicle population distinct form autophagosomes (88).VI. Maintenance of ERES sites

Endoplasmatic reticulum exit sites (ERES) are highly specialized sites that allow the export of proteins into COPII vesicles. In addition to their role in protein export, ERES are involved in the biogenesis of autophagosomes possibly through the delivery of membrane- and other components that are essential in the formation of autophago- 
somes or act as scaffold sites where the autophagosomes may be formed (reviewed in (89)). COPII consists of several protein including the cargo adapters SEC23 and SEC24 (90). In conjunction with lipidated LC3C, Tectonin- $\beta$-propellor containing protein 2 (TECPR2) associates with SEC24D and allows for efficient ER cargo export. Hence, either knockdown of TECPR2 or $L C 3 C$ results in delayed delivery of cargo from the ER to the golgi (91). In addition, the maintenance of ERES via TECPR2 and LC3C is required to autophagosomes formation, failure to maintain ERES sites results in impaired autophagosomes development (91).

Given the high complexity and involvement of the LC3/GABARAP family proteins in these diverse cellular processes, it is likely that these family proteins may be involved in other momentarily unidentified processes. Nevertheless, substantial evidence has shown that the family proteins are also involved in autophagy unrelated processes. Experiments where these family proteins are knocked down should therefore interpreted with caution.

\section{How the LC3/GABARAP family proteins may selectively be recruited}

The LC3/GABARAP protein family fulfills a variety of functions in intracellular trafficking and autophagy. As discussed above, most of these functions appear family protein specific. Interestingly, the functionality and activation of the LC3/GABARAP family proteins at first glance appears to be mediated by comparable enzymatic reactions (terminal glycine exposure, PE-conjugation). To achieve differences and effective recruitment of the required LC3/GABARAP family protein, the cell exploits affinity and specificity differences for ATG proteins which allow complex selection and recruitment of specific LC3/GABARAP family proteins. In this section, the complex recruitment and availability that is required for autophagy execution is discussed.

\section{LC3/GABARAP family protein activation}

LC3/GABARAP family proteins are present in cytoplasmic and membrane-bound isoforms; indicated as -I and -II (for LC3B; LC3B-I and -II) respectively. In yeast, covalent conjugation of a PE-group to Atg8 is required for membrane incorporation. In the Atg8 conjugation reaction, Atg4, Atg7 and Atg3 are crucial(9). For the mammalian orthologues, similar mechanisms have been identified for LC3B, GABARAP, GABARAPL1 and GABARAPL2 (92,93). Whether LC3A and LC3C require ATG4 processing and ATG7 and ATG3 interactions for PE conjugation has not yet been demonstrated.

A series of modifications is required for the efficient conjugation of PE and the correct localization of the LC3/GABARAP family proteins. Members of the ATG4 protease family cleave the pro-form to expose the conserved glycine (isoform-I). Subsequently, the E1 activating enzyme ATG7 catalyzes acyl adenylation of the carboxyl-terminus and 
is then transferred to the E2 conjugating enzyme, ATG3 and, in turn, mediates the covalent conjugation to PE (isoform-II)(94). Although each of the LC3/GABARAP family proteins undergoes this type of conjugation, differences in efficiency and specificity exist. For example, conjugation is most efficient for GABARAPL1, followed by GABARAPL2 and least efficient in LC3B (95). This interpretation is based on the ratio $\mathrm{I} / \mathrm{II}$ in cell-free assays exposing isoform-I to ATP, ATG7, ATG3 and liposomes that are devoid of additional regulatory mechanisms or post-translational modifications. Nevertheless, this setup provides assessment of the systems potential for individual LC3/GABARAP family protein conjugation. These differences indicate that the ratio in LC3/GABARAP family proteins presentation to the ATG7-ATG3 process has a major impact on the generation of membrane associated (-II) forms.

In addition to LC3/GABARAP family protein conjugation, autophagy relies on another conjugation reaction to create the ATG12-ATG5-ATG16(L) complex. For their interaction, ATG12 is activated by the E1 activating enzyme ATG7. Hereafter, ATG12 is transferred to ATG10 (E2 conjugating enzyme) and conjugated to ATG5. ATG5 interacts with ATG16(L) to form the complex, which can form a tetramer via ATG16 homodimerization (reviewed in (96)). The complex fulfils multiple functions. First, it is involved in determination of the site for LC3/GABARAP family protein conjugation to PE by ATG3. In yeast, the Atg12-Atg5 conjugate promotes the transfer of Atg8 from Atg3 to the substrate, PE, by stimulating the activity of Atg3 (8). Also in mammalian cells, localization ATG12ATG5-ATG16L determines the site of LC3B conjugation (97). The direct interaction of ATG12 and ATG3 (98) further indicates that ATG12 recruits ATG3 to determine localization of LC3/GABARAP family protein membrane incorporation. Nevertheless, the ATG12-ATG5 conjugate, but not ATG12 alone, is able to facilitate this LC3/GABARAP family protein PE-conjugation (98). Second, the ATG12-ATG5 conjugate interacts with TECPR1 (a lysosomal resident protein) which is crucial for autophagosome-lysosome fusion (99). Third, the yeast Atg12-Atg5-Atg16 complex and Atg8 cooperate into a selforganizing system to form a protein mesh (scaffold) causing stabilization of the membrane (100). This interaction is mediated through a non-canonical Atg8 binding motif in Atg12, antiparallel arrangement of two Atg16 dimers and the ability of Atg5 to bind membranes and interact with Atg16(L) (100). Interestingly, in mammalian cells GABARAPL1 is observed at the plasma membrane (101) and could indicate a role for GABARAPL1 in membrane stabilization. It is however still unclear whether the ATG12ATG5-ATG16 complex is capable of interacting with other LC3/GABARAP family proteins than LC3B.

The LC3/GABARAP family proteins fulfil unique functions, but appear to be regulated by similar mechanisms. Interestingly, the machinery displays differential conjugation efficiency that can be of importance to orchestrate the functional complexity among the LC3/GABARAP family proteins. 


\section{I.i. ATG4 cysteine protease family - four isoforms varying in homologue selectivity}

Directly after production, pro-LC3B is cleaved at glycine 120 generating isoform I. This priming is comparable for all LC3/GABARAP family proteins and is ATG4 dependent. ATG4 is a class of cysteine proteases with four isoforms in humans; ATG4A, -B, -C and $D$. Most information is available on ATG4B which is capable of cleaving LC3B $(42,102-$ 104), GABARAP (102-104), GABARAPL1 $(42,104)$ and GABARAPL2 $(42,102,103)$. ATG4A displays different substrate specificity and cleaves GABARAPL2 most efficient, followed by GABARAP, and LC3B the least $(102,104)$. Interestingly, overexpression of dominant negative ATG4A mutant (ATG4A ${ }^{C 77 A}$ ) increases the number of unclosed autophagic membranes (17) and is in line with the role for GABARAPL2 in vesicle closure $(17,18)$. Interestingly, ATG4C and ATG4D contain a caspase 3 cleavage domain (DEVD) and, compared to full-length ATG4D, its cleaved form has higher priming and delipidating activity, predominantly directed to GABARAPL1. ATG4D silencing sensitizes HeLa cells to starvation-induced cell death, suggesting that ATG4D-dependent autophagy contributes to cellular survival. Interestingly, cleaved ATG4D is recruited to mitochondria (42) and may mediate recruitment of GABARAPL1 for (dysfunctional) mitochondria clearance (43). Together, this implicates that specific LC3/GABARAP family protein activation and recruitment can be regulated at the post-translational level through activation or expression of ATG4 isoforms.

\section{I.ii. ATG3 and ATG12-ATG5-ATG16(L) dynamics in LC3/GABARAP family protein recruitment}

As LC3/GABARAP family proteins display different functions, recruitment and activation at the site of requirement is therefore important. Directing specific family proteins to the 'flat' membrane or the highly curved extending tips of the phagophore is crucial for elongation and closure. In addition, as LC3/GABARAP family proteins are required for specific types of autophagy, selective recruitment is essential. For example, autophagosomes directed for lysosomal degradation or receptor trafficking from Golgi to the plasma membrane (GABARAP and GABARAPL1-mediated anterograde transport $(57,105))$, requires association with distinct LC3/GABARAP family proteins. This is, at least in part, dependent on ATG3 and ATG12-ATG5-ATG16(L) complex cooperation. Recently, the amino-terminal amphipathic helix in ATG3 has been annotated to sense the membrane curvature (95) and enables ATG3 to associate with highly curved membranes such as the tips of the growing phagophore. Remarkably, the PE-conjugation of GABARAP family proteins by ATG3 increases with the degree of membrane curvature. This allows incorporation of GABARAP family proteins at the phagophore tips and localization of GABARAPL2 at the site of closure (17). This is also illustrated in low-diameter, highly curved liposomes (50-200nm). Here the efficiency in PE-conjugation of 
GABARAPL1, GABARAPL2 and LC3B is different (95). GABARAPL1 protein content is almost completely PE-conjugated at $<100 \mathrm{~nm}$ diameter membranes, whereas this is less effective for GABARAPL2 and LC3B (95). Since classical mammalian autophagosomes are approximately 500-1500nm in size (106), this suggests that GABARAPL1 can be efficiently incorporated in membranes of smaller-sized vesicles (95). The conjugation of GABARAPL1 is highly efficient even in absence of the ATG12-ATG5-ATG16(L) complex, suggesting that for small vesicles the absence of the ATG12-ATG5-ATG16(L) complex would promote incorporation of GABARAPL1 over other family proteins.

Together, increasing evidence is emerging that the cell is able to orchestrate LC3/GABARAP family protein recruitment through the cooperation of ATG3 and the ATG12-ATG5-ATG16(L) complex. Yet, although in yeast an Atg8 binding motif has been identified in Atg12 (100), it remains unclear if this motif exists in mammalian ATG12 and, when exists, has different affinity towards the other LC3/GABARAP family proteins. Potentially, the requirement of this complex for PE-conjugation of LC3B, but not for GABARAPL1 provides the cell with sufficient tools to specifically incorporate the desired LC3/GABARAP family protein into the vesicle.

\section{Transcriptional regulation of LC3/GABARAP family proteins}

Recent reports indicate that transcriptional mechanisms play important roles in the regulation of autophagic activity (107). The diversity of transcription factors that are capable of regulating the expression of LC3/GABARAP family proteins may reflect the requirement for specific family protein expression in e.g. cargo selection or autophagic rate and may depend on environmental factors (metabolic condition, pathogen exposure etc) or may reflect tissue specific effects (table 2).

GATA1/FOXO: The zinc finger DNA binding protein family of GATA (globin transcription factor) transcription factors regulate transcription during development and cell differentiation. GATA binding protein 1 (GATA1) plays an important role in erythropoiesis by controlling the growth and division (proliferation) of immature red blood cells. During erythropoiesis, GATA1 mediates expression of $L C 3 A, \angle C 3 B, G A B A R A P$, GABARAPL1 and GABARAPL2 (108). These effects are, at least in part, mediated through a member of the evolutionary conserved forkhead box O (FOXO) family of transcription factors, FOXO3. During erythropoiesis depletion of FOXO3 reduces the transcriptional effect of GATA1 and decreases upregulation of $\angle C 3 B$, GABARAPL1 and GABARAPL2 (108). In skeletal muscle, FOXO3 is also capable of regulating LC3B and GABARAPL1 expression, whereas in cardiomyocytes, neurons and hepatocytes FOXO1 is also able to fulfill a similar function (109-113).

UPR/ATF4/CHOP: Transcriptional upregulation of LC3/GABARAP family proteins may allow sustained autophagy activity through replenishment of the, during autophagy, degraded protein. For example, the high lysosomal turnover of LC3B during hypoxia- 
induced autophagy in the absence of transcriptional replenishment results in LC3B depletion. Activation of the unfolded protein response (UPR) and production of activating transcription factor 4 (ATF4) (3) results in $\angle C 3 B$ transcription and supports prolonged autophagy(3). In addition, ATF4 controls expression of LC3A, GABARAPL1 and GABARAPL2 in skeletal muscle-, cancer- and embryonic cells respectively $(101,114,115)$. ATF4 induces expression of another transcription factor, CHOP/GADD153/DDIT3/C/EBPhomologues protein, which in turn controls GABARAP expression in embryonic cells (114).

Other regulatory mechanisms: Several other transcription factors have been shown to be able to regulate expression of $L C 3 / G A B A R A P$ family proteins. Most identified factors are capable of increasing expression of the target gene, but also repressive factors have been identified, indicating the importance of gene expression regulation in autophagy. Transcription factor EB (TFEB), a member of the basic helix-loop-helix Leu zipper family has been identified as a key regulating transcription factor for autophagy related genes (107), including LC3B and LC3A $(116,117)$. The extent of gene induction is controlled by the repressive transcription factor zinc-finger protein with KRAB and SCAN domain 3 (ZKSCAN3) (118). During starvation TFEB and ZKSCAN3 are regulated oppositely. Reduction of ZKSCAN expression results in increased $\angle C 3 B$ and GABARAPL2 mRNA expression (118). Whether the repressive effect of ZKSCAN3 on autophagy related genes is exclusively counteracting TFEB-mediated transcription, or that ZKSCAN3 opposes the general transcription of a set of autophagy-related genes, independent of transcription factor, remains elusive.

LC3B is the most studied and therefore best understood member of the LC3/GABARAP family. So far, 8 transcription factors have been identified that are capable of regulating its (basal) expression. In addition to the transcription factors mentioned, LC3B expression is controlled by CCAAT/enhancer binding protein (119), E2F1(120), c-jun $(121,122)$ and sterol regulatory element binding protein 2 (SREBP2) (123). The diversity of stimulatory factors driving $\angle C 3 B$ expression potentially emphasizes the essential role for LC3B in the execution of autophagy. Also for GABARAPL1 many (6) transcription factors have been identified. GABARAPL1 is induced in response to the transcription factors ATF4(101), GATA1(108), C/EBPß(119), FOXO1 (109,112), FOXO3 $(108,111,112)$, and Estrogen receptor activation (124). Furthermore, GABARAP expression is controlled by GATA1 (108), CHOP (114), p3 8 (\$25) and p73 (126) transcriptional activity.

To date no specific transcription factors have been found that regulate LC3C transcription. In clear cell renal cell carcinomas (ccRCC) expression of the tumour suppressor, von Hippel Lindau (VHL) protein, is frequently lost. Loss in VHL, amongst other effects, results in stabilization of hypoxia inducible factor (HIF) proteins. Interestingly, the loss of VHL results in repression of LC3 C mRNA and protein expression. HIF2 a knockdown results in elevated expression of $\mathrm{LC} 3 \mathrm{C}$ in $\mathrm{VHL}$-deficient cells, illustrating the regulation of LC3C expression by VHL-activity through controlling HIF2 a stabilitx(75). 
Table 2: LC3/GABARAP family proteins transcription factors

\begin{tabular}{|c|c|c|c|}
\hline $\begin{array}{l}\text { LC3/GABARAP family } \\
\text { member }\end{array}$ & $\begin{array}{l}\text { Transcription } \\
\text { factor/mechanism }\end{array}$ & Cell type/mechanism & Reference \\
\hline$\angle C 3 A$ & $\begin{array}{l}\text { ATF4 } \\
\text { TFEB } \\
\text { GATA1 }\end{array}$ & $\begin{array}{l}\text { Skeletal muscle } \\
\text { Lung cancer (A549 specifically) } \\
\text { Erythropoiesis }\end{array}$ & $\begin{array}{l}(115) \\
(116) \\
(108)\end{array}$ \\
\hline$\angle C 3 B$ & $\begin{array}{l}\text { ATF4 } \\
\text { C/EBPB } \\
\text { E2F1 } \\
\text { FOXO1 } \\
\text { FOXO3 } \\
\text { GATA1 } \\
\text { C-Jun } \\
\text { SREBP2 } \\
\text { TFEB } \\
\text { ZKSCAN3 (repressive) }\end{array}$ & $\begin{array}{l}\text { Colorectal cancer, glioblastoma, } \\
\text { breast cancer } \\
\text { Liver (circadian rhythm) } \\
\text { Osteosarcoma } \\
\text { Neurons, hepatocytes } \\
\text { Skeletal muscle, erythropoiesis } \\
\text { Erythropoiesis } \\
\text { Nasopharyngeal-, hepatocellular } \\
\text { carcinoma } \\
\text { Human Embryonic kidney } \\
\text { Cervical cancer } \\
\text { Various cancer cell lines }\end{array}$ & $\begin{array}{l}(3) \\
(119) \\
(120) \\
(109,113) \\
(108,110) \\
(108) \\
(121,122) \\
(123) \\
(117) \\
(118)\end{array}$ \\
\hline$\angle C 3 C$ & HIF2 $\alpha$ (repressive) & Clear cell renal cell carcinoma & (75) \\
\hline GABARAP & $\begin{array}{l}\text { CHOP } \\
\text { p3 } 8 \alpha \\
\text { GATA1 } \\
\text { p73 }\end{array}$ & $\begin{array}{l}\text { Mouse embryonic fibroblasts } \\
\text { Colorectal cancer } \\
\text { Erythropoiesis } \\
\text { Rhabdomyosacoma }\end{array}$ & $\begin{array}{l}(114) \\
(125) \\
(108) \\
(126)\end{array}$ \\
\hline GABARAPL1 & $\begin{array}{l}\text { ATF4 } \\
\text { C/EBP } \\
\text { Estrogen-regulated } \\
\text { FOXO1 } \\
\text { FOXO3 } \\
\text { GATA1 }\end{array}$ & $\begin{array}{l}\text { Colorectal cancer, glioblastoma } \\
\text { Liver (circadian rhythm) } \\
\text { Glandular Epithelial Cells } \\
\text { Cardiomyocytes, hepatocytes } \\
\text { Cardiomyocytes, skeletal muscle, } \\
\text { Erythropoiesis } \\
\text { Erythropoiesis }\end{array}$ & $\begin{array}{l}(101) \\
(119) \\
(124) \\
(109,112) \\
(108,111,112) \\
(108)\end{array}$ \\
\hline GABARAPL2 & $\begin{array}{l}\text { ATF4 } \\
\text { GATA1 } \\
\text { ZKSCAN3 (repressive) }\end{array}$ & $\begin{array}{l}\text { Mouse embryonic fibroblasts } \\
\text { Erythropoiesis } \\
\text { Bladder carcinoma }\end{array}$ & $\begin{array}{l}(114) \\
(108) \\
(118)\end{array}$ \\
\hline
\end{tabular}

\section{Post-translational modification}

The best understood post-translational modification of the LC3/GABARAP family protein is the conjugation to PE. This event determines the availability for the family protein to actively participate in autophagy or its related processes. Other post-translational modifications of LC3/GABARAP family proteins are still poorly understood.

Phosphorylation: LC3B phosphorylation by Serine/threonine-protein kinase 3 (STK3) and Serine/threonine-protein kinase 4 (STK4) is important in lysosomal fusion. Failure to phosphorylate LC3B-Thr50 results in failure of fusion of autophagosomes with lysosomes and inhibition of autophagic flux (127). Interestingly, Thr50 is conserved in LC3A$a$ and $-b$ and LC3C, but is absent in the GABARAP family proteins (Fig. 2). In addition, Protein Kinase A (PKA) phosphorylates murine LC3B at serine 12 which restrains LC3B conjugation and autophagosome association. During nutrient depleted conditions PKA activity decreases, leading to rapid activation and availability of LC3B for the execution 
of autophagy (128). Thus, PKA suppresses autophagy and allows existence of a phosphorylated, unconjugated LC3B pool. This pool is then readily available for rapid induction of autophagy. In humans, the $12^{\text {th }}$ amino acid of LC3B is a threonine. Yet, PKA is capable of phosphorylating both serine and threonine residues (129). In LC3A-a, LC3C and GABARAPL2 a serine is observed at position 12 (Fig. 2). While this suggests that the post-translational phosphorylation status of LC3B can regulate the size of the protein pool available for conjugation, it remains unknown, however plausible, if these events regulate other LC3/GABARAP family proteins.

Acetylation: LC3B cycles between nucleus and cytoplasm a process which is regulated by acetylation of LC3B at Lys49 and Lys51 which prevents LC3B from entering the cytoplasm and interaction with ATG7 for further processing. Nutrient deprivation results in deacetylation of nuclear LC3B by sirtuin 1 (SIRT1) leading to an increased cytoplasmic translocation and available substrate for ATG7 (130). Interestingly, both lysine residues at 49 and 51 are present in all LC3/GABARAP family proteins, suggesting a common regulatory mechanism for availability. These insights suggest that post-translational modifications are an important system for regulation LC3B availability and rapid induction of autophagy. If this type of regulation is important for the other LC3/GABARAP family proteins remains to be investigated.

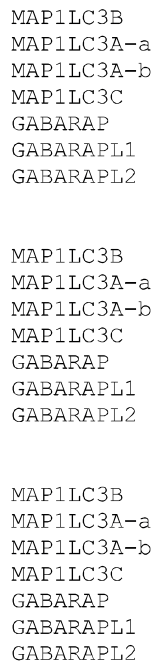

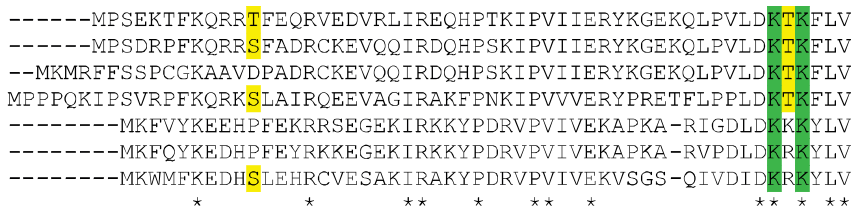

PDHVNMSELIKI IRRRLQLNANQAFFLLVNGHSMVSVSTPISEVYESEKDEDGFLYMVYA PDHVNMSELVKI IRRRLQLNPTQAFFLLVNQHS MVSVST P IADIYEQEKDEDGFLYMVYA PDHVNMSELVKI IRRRLQLNPTQAFFLLVNQHSMVSVSTPIADIYEQEKDEDGFLYMVYA PQELTMTQFLSIIRSRMVLRATEAFYLLVNNKSLVSMSATMAEIYRDYKDEDGFVYMTYA PSDLTVGQFYFIIRKRIHLRAEDALFFFVNNV-IPPTSATMGQLYQEHHEEDFFLYIAYS PSDLTVGQFYFLIRKRIHLRPEDALF FFVNNT - IPPTSATMGQLYEDNHEEDYELYVAYS PSDITVAQFMWI IRKRIQLPSEKAIFLFVDKT-VPQSSLTMGQLYEKEKDEDGELYVAYS

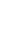




\section{Functional variance by differential (affinity in) binding partners between LC3/GABARAP family proteins}

Besides similarities, functional differences are attributed to LC3/GABARAP family proteins. Important in establishing these differences are the interactions with binding partners. Interestingly, extensive proteomics analysis of binding partners of LC3/GABARAP family proteins has demonstrated that there is overlap in interactome; however, more importantly, that there are clear differences in binding partners. These data are generated based on pull-down assays and will be vital in identifying differences in affinity, specificity in function and biological relevance (37).

The difference in interactome for the LC3/GABARAP family proteins may depend on differential properties of the LC3/GABARAP family proteins. The structure of the LC3/GABARAP family proteins comprises a carboxyl-terminus ubiquitin-like (UBL) core (a conserved ubiquitin fold) and two amino-terminal helices. Binding of the LIR to the LC3/GABARAP family protein occurs at the interface of the amino-terminal arm and the UBL core. The surface of helix 1 of LC3 is basic compared to the acidic nature of GABARAPL2 and GABARAP. The surface of helix 2 is acidic, neutral and basic for respectively LC3, GABARAPL2 and GABARAP. The difference in helices 1 and 2 electrostatic surface potential in LC3/GABARAP family proteins together with differences in the LIR in autophagy receptors contributes to variation in binding partners (10). This is also illustrated by the total number of interacting proteins, of which approximately one-third are specific for LC3 subfamily, one-third specific for GABARAP subfamily and one-third is associated with both (37). An interesting example in this context is LC3C. According to proteomic analysis, LC3C has the least overlapping interactors. Properties of LC3C and its unique interacting partner NDP52 have established its indispensable function in xenophagy.

This emphasizes that subtle differences between proteins allow specific interactions and illustrates the complexity of their functions.

\section{Closing remarks}

There are at least seven orthologues of yeast Atg8 known in humans. Despite the high structural similarities between the LC3/GABARAP family proteins, the existing differences in LIR, amino terminal helices and conformation that makes each family protein unique in binding partners and function. Valuable data on e.g. pull-down assays which contribute to mapping the autophagy interaction network (37) have provided important insights in the interactome. Their roles in autophagy and specific sequestration of cargo are diverse. How the cell orchestrates this complex recruitment to fulfill these specific functions is highlighted in this review. Differences in conjugation (stress-induced regulation; dependent on vesicle curvature), binding partners and expression (via differential 
transcriptional regulation) and post-translational regulation ((de)phosphorylation and (de)acetylation events), contribute to the systems complexity. Increasing evidence indicates that the LC3/GABARAP proteins display functions that are unrelated to canonical autophagy. This has important implications for functional experiments using knockdown models or factors that influence expression of the family proteins. Unravelling the role of the individual LC3/GABARAP family proteins is important to distinguish specialized functions attributed to each specific family protein for autophagy (un)related functions.

\section{Acknowledgements}

The authors would like to apologize to authors who's work could not be included due to the extensive field of autophagy. This work was financially supported by the Dutch Cancer Society (KWF Grants UM 2012-5506 and 2015-7735 to K.R.), worldwide cancer research award 16-0265 (to K.R.) and European Research Council under the European Union's Seventh Framework Programme (FP/2007-2013)/ERC Grant Agreement (n. 617060 to M.V.). 


\section{References}

1. Rabinowitz JD, White E. Autophagy and metabolism. Science 2010;330:1344-8

2. Marx J. Autophagy: is it cancer's friend or foe? Science 2006;312:1160-1

3. Rouschop KM, van den Beucken T, Dubois L, Niessen H, Bussink J, Savelkouls K, et al. The unfolded protein response protects human tumor cells during hypoxia through regulation of the autophagy genes MAP1LC3B and ATG5. J Clin Invest 2010;120:127-41

4. Levine B, Kroemer G. Autophagy in the pathogenesis of disease. Cell 2008;132:27-42

5. Komatsu M, Waguri S, Chiba T, Murata S, Iwata J, Tanida I, et al. Loss of autophagy in the central nervous system causes neurodegeneration in mice. Nature 2006;441:880-4

6. Komatsu M, Waguri S, Ueno T, Iwata J, Murata S, Tanida I, et al. Impairment of starvation-induced and constitutive autophagy in Atg7-deficient mice. J Cell Biol 2005;169:425-34

7. Xie Z, Nair U, Klionsky DJ. Atg8 controls phagophore expansion during autophagosome formation. Molecular biology of the cell 2008;19:3290-8

8. Shpilka T, Mizushima N, Elazar Z. Ubiquitin-like proteins and autophagy at a glance. Journal of cell science 2012;125:2343-8

9. Ichimura Y, Kirisako T, Takao T, Satomi Y, Shimonishi Y, Ishihara N, et al. A ubiquitin-like system mediates protein lipidation. Nature 2000;408:488-92

10. Sugawara K, Suzuki NN, Fujioka Y, Mizushima N, Ohsumi Y, Inagaki F. The crystal structure of microtubule-associated protein light chain 3, a mammalian homologue of Saccharomyces cerevisiae Atg8. Genes to cells : devoted to molecular \& cellular mechanisms 2004;9:611-8

11. Lamb CA, Yoshimori T, Tooze SA. The autophagosome: origins unknown, biogenesis complex. Nature reviews Molecular cell biology 2013;14:759-74

12. Shang L, Chen S, Du F, Li S, Zhao L, Wang X. Nutrient starvation elicits an acute autophagic response mediated by Ulk1 dephosphorylation and its subsequent dissociation from AMPK. Proceedings of the National Academy of Sciences of the United States of America 2011;108:4788-93

13. Schaaf MB, Cojocari D, Keulers TG, Jutten B, Starmans MH, de Jong MC, et al. The autophagy associated gene, ULK1, promotes tolerance to chronic and acute hypoxia. Radiother Oncol 2013;108:529-34

14. Axe EL, Walker SA, Manifava M, Chandra P, Roderick HL, Habermann A, et al. Autophagosome formation from membrane compartments enriched in phosphatidylinositol 3-phosphate and dynamically connected to the endoplasmic reticulum. The Journal of cell biology 2008;182:685-701

15. Burman C, Ktistakis NT. Regulation of autophagy by phosphatidylinositol 3-phosphate. FEBS Lett 2010;584:1302-12

16. Alemu EA, Lamark T, Torgersen KM, Birgisdottir AB, Larsen KB, Jain A, et al. ATG8 family proteins act as scaffolds for assembly of the ULK complex: sequence requirements for LC3-interacting region (LIR) motifs. J Biol Chem 2012;287:39275-90

17. Weidberg H, Shvets E, Shpilka T, Shimron F, Shinder V, Elazar Z. LC3 and GATE-16/GABARAP subfamilies are both essential yet act differently in autophagosome biogenesis. The EMBO journal 2010;29:1792-802

18. Weidberg H, Shpilka T, Shvets E, Abada A, Shimron F, Elazar Z. LC3 and GATE-16 N termini mediate membrane fusion processes required for autophagosome biogenesis. Developmental cell 2011;20:444-54

19. Coyle JE, Qamar S, Rajashankar KR, Nikolov DB. Structure of GABARAP in two conformations: implications for GABA(A) receptor localization and tubulin binding. Neuron 2002;33:63-74

20. Wild P, McEwan DG, Dikic I. The LC3 interactome at a glance. Journal of cell science 2014;127:3-9

21. Pankiv S, Clausen TH, Lamark T, Brech A, Bruun JA, Outzen H, et al. p62/SOSTM1 binds directly to Atg8/LC3 to facilitate degradation of ubiquitinated protein aggregates by autophagy. J Biol Chem 2007;282:24131-45

22. Shvets E, Abada A, Weidberg H, Elazar Z. Dissecting the involvement of LC3B and GATE-16 in p62 recruitment into autophagosomes. Autophagy 2011;7:683-8

23. Clausen TH, Lamark T, Isakson P, Finley K, Larsen KB, Brech A, et al. p62/SOSTM1 and ALFY interact to facilitate the formation of $\mathrm{p} 62$ bodies/ALIS and their degradation by autophagy. Autophagy 2010;6:330-44 
24. Lystad AH, Ichimura Y, Takagi K, Yang Y, Pankiv S, Kanegae Y, et al. Structural determinants in GABARAP required for the selective binding and recruitment of ALFY to LC3B-positive structures. EMBO reports 2014;15:557-65

25. Lu K, Psakhye I, Jentsch S. Autophagic clearance of polyQ proteins mediated by ubiquitin-Atg8 adaptors of the conserved CUET protein family. Cell 2014;158:549-63

26. Thurston TL, Wandel MP, von Muhlinen N, Foeglein A, Randow F. Galectin 8 targets damaged vesicles for autophagy to defend cells against bacterial invasion. Nature 2012;482:414-8

27. Verlhac P, Viret C, Faure M. Dual function of CALCOCO2/NDP52 during xenophagy. Autophagy 2015;11:965-6

28. Verlhac P, Gregoire IP, Azocar O, Petkova DS, Baguet J, Viret C, et al. Autophagy receptor NDP52 regulates pathogen-containing autophagosome maturation. Cell Host Microbe 2015;17:515-25

29. von Muhlinen N, Akutsu M, Ravenhill BJ, Foeglein A, Bloor S, Rutherford TJ, et al. LC3C, bound selectively by a noncanonical LIR motif in NDP52, is required for antibacterial autophagy. Molecular cell 2012;48:329-42

30. Wild P, Farhan H, McEwan DG, Wagner S, Rogov VV, Brady NR, et al. Phosphorylation of the autophagy receptor optineurin restricts Salmonella growth. Science 2011;333:228-33

31. Dupont N, Lacas-Gervais S, Bertout J, Paz I, Freche B, Van Nhieu GT, et al. Shigella phagocytic vacuolar membrane remnants participate in the cellular response to pathogen invasion and are regulated by autophagy. Cell Host Microbe 2009;6:137-49

32. Mostowy S, Sancho-Shimizu V, Hamon MA, Simeone R, Brosch R, Johansen T, et al. p62 and NDP52 proteins target intracytosolic Shigella and Listeria to different autophagy pathways. J Biol Chem 2011;286:26987-95

33. Tumbarello DA, Manna PT, Allen M, Bycroft M, Arden SD, Kendrick-Jones J, et al. The Autophagy Receptor TAX1BP1 and the Molecular Motor Myosin VI Are Required for Clearance of Salmonella Typhimurium by Autophagy. PLoS Pathog 2015;11:e1005174

34. Mandell MA, Kimura T, Jain A, Johansen T, Deretic V. TRIM proteins regulate autophagy: TRIM5 is a selective autophagy receptor mediating HIV-1 restriction. Autophagy 2014;10:2387-8

35. Malicdan MC, Nishino I. Autophagy in lysosomal myopathies. Brain Pathol 2012;22:82-8

36. Jiang S, Heller B, Tagliabracci VS, Zhai L, Irimia JM, DePaoli-Roach AA, et al. Starch binding domaincontaining protein 1/genethonin 1 is a novel participant in glycogen metabolism. J Biol Chem 2010;285:34960-71

37. Behrends C, Sowa ME, Gygi SP, Harper JW. Network organization of the human autophagy system. Nature 2010;466:68-76

38. Jiang S, Wells CD, Roach PJ. Starch-binding domain-containing protein 1 (Stbd1) and glycogen metabolism: Identification of the Atg8 family interacting motif (AIM) in Stbd1 required for interaction with GABARAPL1. Biochemical and biophysical research communications 2011;413:420-5

39. Rouschop KMA, Ramaekers CHMA, Schaaf MBE, Keulers TGH, Savelkouls KGM, Lambin P, et al. Autophagy is required during cycling hypoxia to lower production of reactive oxygen species. Radiotherapy and Oncology 2009;92:411-6

40. Lazarou M, Sliter DA, Kane LA, Sarraf SA, Wang C, Burman JL, et al. The ubiquitin kinase PINK1 recruits autophagy receptors to induce mitophagy. Nature 2015;524:309-14

41. Novak I, Kirkin V, McEwan DG, Zhang J, Wild P, Rozenknop A, et al. Nix is a selective autophagy receptor for mitochondrial clearance. EMBO reports 2010;11:45-51

42. Betin VM, Lane JD. Caspase cleavage of Atg4D stimulates GABARAP-L1 processing and triggers mitochondrial targeting and apoptosis. Journal of cell science 2009;122:2554-66

43. Boyer-Guittaut M, Poillet L, Liang $Q$, Bole-Richard E, Ouyang X, Benavides GA, et al. The role of GABARAPL1/GEC1 in autophagic flux and mitochondrial quality control in MDA-MB-436 breast cancer cells. Autophagy 2014;10:986-1003

44. Liu L, Feng D, Chen G, Chen M, Zheng Q, Song P, et al. Mitochondrial outer-membrane protein FUNDC1 mediates hypoxia-induced mitophagy in mammalian cells. Nat Cell Biol 2012;14:177-85 
45. Zhu Y, Massen S, Terenzio M, Lang V, Chen-Lindner S, Eils R, et al. Modulation of serines 17 and 24 in the LC3-interacting region of Bnip3 determines pro-survival mitophagy versus apoptosis. J Biol Chem 2013;288:1099-113

46. Fader CM, Colombo MI. Autophagy and multivesicular bodies: two closely related partners. Cell death and differentiation 2009;16:70-8

47. Jager S, Bucci C, Tanida I, Ueno T, Kominami E, Saftig P, et al. Role for Rab7 in maturation of late autophagic vacuoles. Journal of cell science 2004;117:4837-48

48. Ishihara N, Hamasaki M, Yokota S, Suzuki K, Kamada Y, Kihara A, et al. Autophagosome requires specific early Sec proteins for its formation and NSF/SNARE for vacuolar fusion. Molecular biology of the cell 2001;12:3690-702

49. McNew JA, Weber T, Parlati F, Johnston RJ, Melia TJ, Sollner TH, et al. Close is not enough: SNAREdependent membrane fusion requires an active mechanism that transduces force to membrane anchors. The Journal of cell biology 2000;150:105-17

50. Thielmann Y, Weiergraber OH, Ma P, Schwarten M, Mohrluder J, Willbold D. Comparative modeling of human NSF reveals a possible binding mode of GABARAP and GATE-16. Proteins 2009;77:637-46

51. Sagiv Y, Legesse-Miller A, Porat A, Elazar Z. GATE-16, a membrane transport modulator, interacts with NSF and the Golgi v-SNARE GOS-28. The EMBO journal 2000;19:1494-504

52. Choi S, Kim HJ. The Ca2+ channel TRPML3 specifically interacts with the mammalian ATG8 homologue GATE16 to regulate autophagy. Biochemical and biophysical research communications 2014;443:56-61

53. Chanturiya A, Scaria P, Woodle MC. The role of membrane lateral tension in calcium-induced membrane fusion. The Journal of membrane biology 2000;176:67-75

54. McEwan DG, Popovic D, Gubas A, Terawaki S, Suzuki H, Stadel D, et al. PLEKHM1 Regulates Autophagosome-Lysosome Fusion through HOPS Complex and LC3/GABARAP Proteins. Mol Cell 2015; $57: 39-54$

55. Jacob TC, Moss SJ, Jurd R. GABA(A) receptor trafficking and its role in the dynamic modulation of neuronal inhibition. Nat Rev Neurosci 2008;9:331-43

56. Cook JL, Re RN, deHaro DL, Abadie JM, Peters M, Alam J. The trafficking protein GABARAP binds to and enhances plasma membrane expression and function of the angiotensin II type 1 receptor. Circ Res 2008;102:1539-47

57. Alam J, Deharo D, Redding KM, Re RN, Cook JL. C-terminal processing of GABARAP is not required for trafficking of the angiotensin II type 1A receptor. Regul Pept 2010;159:78-86

58. Chen C, Wang Y, Huang P, Liu-Chen LY. Effects of C-terminal modifications of GEC1 protein and gammaaminobutyric acid type $A(G A B A(A))$ receptor-associated protein (GABARAP), two microtubule-associated proteins, on kappa opioid receptor expression. J Biol Chem 2011;286:15106-15

59. Lainez S, Valente P, Ontoria-Oviedo I, Estevez-Herrera J, Camprubi-Robles M, Ferrer-Montiel A, et al. GABAA receptor associated protein (GABARAP) modulates TRPV1 expression and channel function and desensitization. Faseb J 2010;24:1958-70

60. O'Sullivan GA, Kneussel M, Elazar Z, Betz H. GABARAP is not essential for GABA receptor targeting to the synapse. Eur J Neurosci 2005;22:2644-8

61. Nakajima K, Yin X, Takei Y, Seog DH, Homma N, Hirokawa N. Molecular motor KIF5A is essential for GABA(A) receptor transport, and KIF5A deletion causes epilepsy. Neuron 2012;76:945-61

62. Labonte $D$, Thies $E$, Kneussel M. The kinesin KIF21B participates in the cell surface delivery of gamma2 subunit-containing GABAA receptors. Eur J Cell Biol 2014;93:338-46

63. Monastyrska I, Rieter E, Klionsky DJ, Reggiori F. Multiple roles of the cytoskeleton in autophagy. Biol Rev Camb Philos Soc 2009;84:431-48

64. Mansuy V, Boireau W, Fraichard A, Schlick JL, Jouvenot M, Delage-Mourroux R. GEC1, a protein related to GABARAP, interacts with tubulin and GABA(A) receptor. Biochem Biophys Res Commun 2004;325:639-48

65. Mohrluder J, Stangler T, Hoffmann Y, Wiesehan K, Mataruga A, Willbold D. Identification of calreticulin as a ligand of GABARAP by phage display screening of a peptide library. Febs J 2007;274:5543-55 
66. Kittler JT, Rostaing P, Schiavo G, Fritschy JM, Olsen R, Triller A, et al. The subcellular distribution of GABARAP and its ability to interact with NSF suggest a role for this protein in the intracellular transport of GABA(A) receptors. Mol Cell Neurosci 2001;18:13-25

67. Kneussel M, Brandstatter JH, Laube B, Stahl S, Muller U, Betz H. Loss of postsynaptic GABA(A) receptor clustering in gephyrin-deficient mice. J Neurosci 1999;19:9289-97

68. Leil TA, Chen ZW, Chang CS, Olsen RW. GABAA receptor-associated protein traffics GABAA receptors to the plasma membrane in neurons. J Neurosci 2004;24:11429-38

69. Jacob TC, Bogdanov YD, Magnus C, Saliba RS, Kittler JT, Haydon PG, et al. Gephyrin regulates the cell surface dynamics of synaptic GABAA receptors. J Neurosci 2005;25:10469-78

70. Kneussel M, Haverkamp S, Fuhrmann JC, Wang H, Wassle H, Olsen RW, et al. The gamma-aminobutyric acid type $A$ receptor (GABAAR)-associated protein GABARAP interacts with gephyrin but is not involved in receptor anchoring at the synapse. Proc Natl Acad Sci U S A 2000;97:8594-9

71. Keulers TG, Schaaf MB, Peeters HJ, Savelkouls KG, Vooijs MA, Bussink J, et al. GABARAPL1 is required for increased EGFR membrane expression during hypoxia. Radiother Oncol 2015;116:417-22

72. Legesse-Miller A, Sagiv Y, Porat A, Elazar Z. Isolation and characterization of a novel low molecular weight protein involved in intra-Golgi traffic. J Biol Chem 1998;273:3105-9

73. Bai H, Inoue J, Kawano T, Inazawa J. A transcriptional variant of the LC3A gene is involved in autophagy and frequently inactivated in human cancers. Oncogene 2012;31:4397-408

74. Cowey CL, Rathmell WK. VHL Gene Mutations in Renal Cell Carcinoma: Role as a Biomarker of Disease Outcome and Drug Efficacy. Current oncology reports 2009;11:94-101

75. Mikhaylova O, Stratton Y, Hall D, Kellner E, Ehmer B, Drew AF, et al. VHL-regulated MiR-204 suppresses tumor growth through inhibition of LC3B-mediated autophagy in renal clear cell carcinoma. Cancer Cell 2012;21:532-46

76. Wu S, Sun C, Tian D, Li Y, Gao X, He S, et al. Expression and clinical significances of Beclin1, LC3 and mTOR in colorectal cancer. Int J Clin Exp Pathol 2015;8:3882-91

77. Klebig C, Seitz S, Arnold W, Deutschmann N, Pacyna-Gengelbach M, Scherneck S, et al. Characterization of \{gamma\}-aminobutyric acid type A receptor-associated protein, a novel tumor suppressor, showing reduced expression in breast cancer. Cancer research 2005;65:394-400

78. Salah FS, Ebbinghaus M, Muley VY, Zhou Z, Al-Saadi KR, Pacyna-Gengelbach M, et al. Tumor suppression in mice lacking GABARAP, an Atg8/LC3 family member implicated in autophagy, is associated with alterations in cytokine secretion and cell death. Cell death \& disease 2016;7:e2205

79. Wells A, Welsh JB, Lazar CS, Wiley HS, Gill GN, Rosenfeld MG. Ligand-induced transformation by a noninternalizing epidermal growth factor receptor. Science 1990;247:962-4

80. Cali T, Galli C, Olivari S, Molinari M. Segregation and rapid turnover of EDEM1 by an autophagy-like mechanism modulates standard ERAD and folding activities. Biochemical and biophysical research communications 2008;371:405-10

81. Sanjuan MA, Dillon CP, Tait SW, Moshiach S, Dorsey F, Connell S, et al. Toll-like receptor signalling in macrophages links the autophagy pathway to phagocytosis. Nature 2007;450:1253-7

82. Huang J, Canadien V, Lam GY, Steinberg BE, Dinauer MC, Magalhaes MA, et al. Activation of antibacterial autophagy by NADPH oxidases. Proceedings of the National Academy of Sciences of the United States of America 2009;106:6226-31

83. Martinez J, Almendinger J, Oberst A, Ness R, Dillon CP, Fitzgerald P, et al. Microtubule-associated protein 1 light chain 3 alpha (LC3)-associated phagocytosis is required for the efficient clearance of dead cells. Proceedings of the National Academy of Sciences of the United States of America 2011;108:17396-401

84. Martinez J, Malireddi RK, Lu Q, Cunha LD, Pelletier S, Gingras S, et al. Molecular characterization of LC3associated phagocytosis reveals distinct roles for Rubicon, NOX2 and autophagy proteins. Nature cell biology 2015;17:893-906

85. Martinez J, Cunha LD, Park S, Yang M, Lu Q, Orchard R, et al. Noncanonical autophagy inhibits the autoinflammatory, lupus-like response to dying cells. Nature 2016;533:115-9

86. Cemma M, Grinstein S, Brumell JH. Autophagy proteins are not universally required for phagosome maturation. Autophagy 2016:1-7 
87. Romao S, Gasser N, Becker AC, Guhl B, Bajagic M, Vanoaica D, et al. Autophagy proteins stabilize pathogen-containing phagosomes for prolonged $\mathrm{MHC}$ II antigen processing. The Journal of cell biology 2013;203:757-66

88. Genau HM, Huber J, Baschieri F, Akutsu M, Dotsch V, Farhan H, et al. CUL3-KBTBD6/KBTBD7 ubiquitin ligase cooperates with GABARAP proteins to spatially restrict TIAM1-RAC1 signaling. Mol Cell 2015;57:995-1010

89. Sanchez-Wandelmer J, Ktistakis NT, Reggiori F. ERES: sites for autophagosome biogenesis and maturation? J Cell Sci 2015;128:185-92

90. Jensen D, Schekman R. COPII-mediated vesicle formation at a glance. Journal of cell science 2011;124:1-4

91. Stadel D, Millarte V, Tillmann KD, Huber J, Tamin-Yecheskel BC, Akutsu M, et al. TECPR2 Cooperates with LC3C to Regulate COPII-Dependent ER Export. Molecular cell 2015;60:89-104

92. Nath S, Dancourt J, Shteyn V, Puente G, Fong WM, Nag S, et al. Lipidation of the LC3/GABARAP family of autophagy proteins relies on a membrane-curvature-sensing domain in Atg3. Nature cell biology 2014;16:415-24

93. Sou YS, Tanida I, Komatsu M, Ueno T, Kominami E. Phosphatidylserine in addition to phosphatidylethanolamine is an in vitro target of the mammalian Atg8 modifiers, LC3, GABARAP, and GATE-16. J Biol Chem 2006;281:3017-24

94. Tanida I, Ueno T, Kominami E. LC3 conjugation system in mammalian autophagy. The international journal of biochemistry \& cell biology 2004;36:2503-18

95. Nath S, Dancourt J, Shteyn V, Puente G, Fong WM, Nag S, et al. Lipidation of the LC3/GABARAP family of autophagy proteins relies on a membrane-curvature-sensing domain in Atg3. Nat Cell Biol 2014;16:415-24

96. Geng J, Klionsky DJ. The Atg8 and Atg12 ubiquitin-like conjugation systems in macroautophagy. 'Protein modifications: beyond the usual suspects' review series. EMBO reports 2008;9:859-64

97. Fujita N, Itoh T, Omori H, Fukuda M, Noda T, Yoshimori T. The Atg16L complex specifies the site of LC3 lipidation for membrane biogenesis in autophagy. Mol Biol Cell 2008;19:2092-100

98. Otomo C, Metlagel Z, Takaesu G, Otomo T. Structure of the human ATG12 ATG5 conjugate required for LC3 lipidation in autophagy. Nat Struct Mol Biol 2013;20:59-66

99. Chen D, Fan W, Lu Y, Ding X, Chen S, Zhong Q. A mammalian autophagosome maturation mechanism mediated by TECPR1 and the Atg12-Atg5 conjugate. Mol Cell 2012;45:629-41

100. Kaufmann A, Beier V, Franquelim HG, Wollert T. Molecular mechanism of autophagic membrane-scaffold assembly and disassembly. Cell 2014;156:469-81

101. Keulers TG, Schaaf MB, Peeters HJ, Savelkouls KG, Vooijs MA, Bussink J, et al. GABARAPL1 is required for increased EGFR membrane expression during hypoxia. Radiotherapy and oncology : journal of the European Society for Therapeutic Radiology and Oncology 2015

102. Kabeya Y, Mizushima N, Yamamoto A, Oshitani-Okamoto S, Ohsumi Y, Yoshimori T. LC3, GABARAP and GATE16 localize to autophagosomal membrane depending on form-ll formation. J Cell Sci 2004;117:2805-12

103. Tanida I, Sou YS, Ezaki J, Minematsu-Ikeguchi N, Ueno T, Kominami E. HsAtg4B/HsApg4B/autophagin-1 cleaves the carboxyl termini of three human Atg8 homologues and delipidates microtubule-associated protein light chain 3- and GABAA receptor-associated protein-phospholipid conjugates. The Journal of biological chemistry 2004;279:36268-76

104. Zhang Y, Wang F, Han L, Wu Y, Li S, Yang X, et al. GABARAPL1 negatively regulates Wnt/beta-catenin signaling by mediating Dvl2 degradation through the autophagy pathway. Cell Physiol Biochem 2011;27:503-12

105. Chen ZW, Chang CS, Leil TA, Olsen RW. C-terminal modification is required for GABARAP-mediated GABA(A) receptor trafficking. J Neurosci 2007;27:6655-63

106. Mizushima N, Ohsumi Y, Yoshimori T. Autophagosome formation in mammalian cells. Cell Struct Funct 2002;27:421-9

107. Fullgrabe J, Klionsky DJ, Joseph B. The return of the nucleus: transcriptional and epigenetic control of autophagy. Nat Rev Mol Cell Biol 2014;15:65-74 
108. Kang YA, Sanalkumar R, O'Geen H, Linnemann AK, Chang CJ, Bouhassira EE, et al. Autophagy driven by a master regulator of hematopoiesis. Mol Cell Biol 2012;32:226-39

109. Liu HY, Han J, Cao SY, Hong T, Zhuo D, Shi J, et al. Hepatic autophagy is suppressed in the presence of insulin resistance and hyperinsulinemia: inhibition of FoxO1-dependent expression of key autophagy genes by insulin. J Biol Chem 2009;284:31484-92

110. Mammucari C, Milan G, Romanello V, Masiero E, Rudolf R, Del Piccolo P, et al. FoxO3 controls autophagy in skeletal muscle in vivo. Cell Metab 2007;6:458-71

111. Sanchez AM, Csibi A, Raibon A, Cornille K, Gay S, Bernardi H, et al. AMPK promotes skeletal muscle autophagy through activation of forkhead FoxO3a and interaction with Ulk1. J Cell Biochem 2012;113:695-710

112. Sengupta A, Molkentin JD, Yutzey KE. FoxO transcription factors promote autophagy in cardiomyocytes. J Biol Chem 2009;284:28319-31

113. Xu P, Das M, Reilly J, Davis RJ. JNK regulates FoxO-dependent autophagy in neurons. Genes Dev 2011;25:310-22

114. B'chir W, Maurin AC, Carraro V, Averous J, Jousse C, Muranishi Y, et al. The elF2 alpha/ATF4 pathway is essential for stress-induced autophagy gene expression. Nucleic Acids Res 2013;41:7683-99

115. Wang H, Wilson GJ, Zhou D, Lezmi S, Chen X, Layman DK, et al. Induction of autophagy through the activating transcription factor 4 (ATF4)-dependent amino acid response pathway in maternal skeletal muscle may function as the molecular memory in response to gestational protein restriction to alert offspring to maternal nutrition. Br J Nutr 2015:1-14

116. Giatromanolaki A, Kalamida D, Sivridis E, Karagounis IV, Gatter KC, Harris AL, et al. Increased expression of transcription factor EB (TFEB) is associated with autophagy, migratory phenotype and poor prognosis in non-small cell lung cancer. Lung Cancer 2015;90:98-105

117. Settembre C, De Cegli R, Mansueto G, Saha PK, Vetrini F, Visvikis O, et al. TFEB controls cellular lipid metabolism through a starvation-induced autoregulatory loop. Nature cell biology 2013;15:647-58

118. Chauhan S, Goodwin JG, Manyam G, Wang J, Kamat AM, Boyd DD. ZKSCAN3 is a master transcriptional repressor of autophagy. Mol Cell 2013;50:16-28

119. Ma D, Panda S, Lin JD. Temporal orchestration of circadian autophagy rhythm by C/EBPbeta. The EMBO journal 2011;30:4642-51

120. Polager S, Ofir M, Ginsberg D. E2F1 regulates autophagy and the transcription of autophagy genes. Oncogene 2008;27:4860-4

121. Li DD, Wang LL, Deng R, Tang J, Shen Y, Guo JF, et al. The pivotal role of c-Jun NH2-terminal kinasemediated Beclin 1 expression during anticancer agents-induced autophagy in cancer cells. Oncogene 2009;28:886-98

122. Sun T, Li D, Wang L, Xia L, Ma J, Guan Z, et al. c-Jun NH2-terminal kinase activation is essential for upregulation of LC3 during ceramide-induced autophagy in human nasopharyngeal carcinoma cells. J Transl Med 2011;9:161

123. Seo YK, Jeon TI, Chong HK, Biesinger J, Xie X, Osborne TF. Genome-wide localization of SREBP-2 in hepatic chromatin predicts a role in autophagy. Cell metabolism 2011;13:367-75

124. Vernier-Magnin S, Nemos C, Mansuy V, Tolle F, Guichard L, Delage-Mourroux R, et al. Analysis of the guinea-pig estrogen-regulated gec1/GABARAPL1 gene promoter and identification of a functional ERE in the first exon. Biochim Biophys Acta 2005;1731:23-31

125. Comes F, Matrone A, Lastella P, Nico B, Susca FC, Bagnulo R, et al. A novel cell type-specific role of p38alpha in the control of autophagy and cell death in colorectal cancer cells. Cell Death Differ 2007;14:693-702

126. Rosenbluth JM, Pietenpol JA. mTOR regulates autophagy-associated genes downstream of p73. Autophagy 2009;5:114-6

127. Wilkinson DS, Jariwala JS, Anderson E, Mitra K, Meisenhelder J, Chang JT, et al. Phosphorylation of LC3 by the Hippo kinases STK3/STK4 is essential for autophagy. Molecular cell 2015;57:55-68

128. Cherra SJ, 3rd, Kulich SM, Uechi G, Balasubramani M, Mountzouris J, Day BW, et al. Regulation of the autophagy protein LC3 by phosphorylation. The Journal of cell biology 2010;190:533-9 
129. Shabb JB. Physiological substrates of cAMP-dependent protein kinase. Chem Rev 2001;101:2381-411

130. Huang R, Xu Y, Wan W, Shou X, Qian J, You Z, et al. Deacetylation of nuclear LC3 drives autophagy initiation under starvation. Mol Cell 2015;57:456-66

131. Pankiv S, Alemu EA, Brech A, Bruun JA, Lamark T, Overvatn A, et al. FYCO1 is a Rab7 effector that binds to LC3 and PI3P to mediate microtubule plus end-directed vesicle transport. The Journal of cell biology 2010;188:253-69

132. McEwan DG, Popovic D, Gubas A, Terawaki S, Suzuki H, Stadel D, et al. PLEKHM1 Regulates Autophagosome-Lysosome Fusion through HOPS Complex and LC3/GABARAP Proteins. Molecular cell 2015;57:39-54

133. Kirkin V, Lamark T, Sou YS, Bjorkoy G, Nunn JL, Bruun JA, et al. A role for NBR1 in autophagosomal degradation of ubiquitinated substrates. Molecular cell 2009;33:505-16

134. Genau HM, Huber J, Baschieri F, Akutsu M, Dotsch V, Farhan H, et al. CUL3-KBTBD6/KBTBD7 Ubiquitin Ligase Cooperates with GABARAP Proteins to Spatially Restrict TIAM1-RAC1 Signaling. Mol Cell 2015 
Chapter

\section{GABARAPL1 is required for increased EGFR membrane expression during hypoxia}

Tom G. Keulers ${ }^{1 *}$, Marco B.E. Schaaf ${ }^{1 *}$, Hanneke J.M. Peeters ${ }^{1}$, Kim G.M. Savelkouls ${ }^{1}$, Marc A. Vooijs ${ }^{1}$, Johan Bussink ${ }^{2}$, Barry Jutten ${ }^{1}$, Kasper M.A. Rouschop ${ }^{1}$

${ }^{1}$ Maastricht Radiation Oncology (MaastRO) lab, GROW - School for Oncology and Developmental Biology, Maastricht University, Maastricht, the Netherlands ${ }^{2}$ Department of Radiation Oncology, Radboud University Medical Center, Nijmegen, the Netherlands

*contributed equally

Published in Radiother Oncol. 2015 Sep;116(3):417-22. 


\section{Abstract}

Background and purpose The epidermal growth factor receptor (EGFR) is overexpressed, amplified or mutated in various human epithelial tumours and hypoxia is a common feature of solid tumours. Both EGFR and hypoxia are associated with therapy resistance and poor treatment outcome. To survive hypoxia, cells adapt by activation of hypoxia responsive pathways and expression of hypoxia-induced plasma membrane proteins. We observed that $\mathrm{GABA}_{A}$ receptor associated protein like1 (GABARAPL1) and plasma membrane expression of EGFR were increased during hypoxia. Here we explored the role of the GABARAPL1 in EGFR membrane expression during hypoxia.

Material and methods Quantitative qPCR, immunoblot analysis, flow cytometry and cytochemistry were used to assess this interplay.

Results GABARAPL1 mRNA and protein levels are increased during hypoxia in vitro and correlate with tumour hypoxia in a panel of primary HNSCC xenografts. High GABARAPL1 mRNA is associated with poor outcome of HNSCC patients. During hypoxia, EGFR membrane expression is increased and GABARAPL1 and EGFR colocalize at the plasma membrane. GABARAPL1 knockdown inhibits EGFR membrane expression during hypoxia.

Conclusion GABARAPL1 is required for increased membrane expression of EGFR during hypoxia, suggesting a role for GABARAPL1 in the trafficking of these membrane proteins. 


\section{Introduction}

Solid tumours are characterized by the presence of abnormal and immature vasculature that leads to extreme homogeneities in oxygenation. Oxygenation in these regions varies from normal (approx. 5\% $\mathrm{O}_{2}$ ) to severe hypoxia and even anoxia [1, 2]. Tumour cells within hypoxic regions contribute to increased invasion [3], metastatic spread [4] and therapy resistance [5].

For survival, cancer cells adapt to hypoxia by activation of several signalling pathways including the hypoxia inducible factor (HIF) pathway and the unfolded protein response (UPR). During normoxic conditions, the HIF-1 a protein isubiquitylated and rapidly degraded. HIF1 $\alpha$ istabilized during hypoxia and modifies the microenvironment by upregulating expression of several proteins. For example, through increasing vascular endothelial growth factor (VEGF) expression and induction of angiogenesis [6]. Other HIF1 transcriptional targets are carbonic anhydrase 9 (CA9) and Glucose Transporter 1 (GLUT1). These proteins are respectively involved in deacidification and increased glucose uptake during hypoxia [7].

The UPR is activated during hypoxia as a consequence of increased misfolded or unfolded proteins [8]. The UPR constitutes of three parallel signalling cascades initiated by PERK (PKR-like ER kinase), IRE1 (inositol-requiring protein 1) and ATF6 (activating transcription factor 6 ). PERK is a kinase that phosphorylates elF2 $\alpha$ to inhibit translation initiation. The blockade in overall protein translation allows selective translation of the transcription factor, ATF4 [9], and subsequently CHOP [10]. The UPR is important for inhibition of mRNA translation [11], promote ER homeostasis [12], increasing the cell's antioxidant capacity [13] and induce expression of several autophagy-related genes [14] [15] during hypoxia.

Epidermal growth factor receptor (EGFR) signalling is increased in many cancer types due to overexpression, amplification or mutation. Activation of the receptor leads to a cascade of signalling pathways that control growth, migration and differentiation and acquisition of an EMT-like phenotype [16]. EGFR is upregulated during hypoxia [17] and combined targeting of EGFR and hypoxia modification is expected to increase tumour responsiveness to radiotherapy [18].

After production, membrane proteins, including EGFR, require active transportation to the plasma membrane. GABARAPL1 is a member of the GABARAP protein family, which consists of 3 members, GABARAP, GABARAPL1 and gate-16/GABARAPL2. Although their function is largely unknown, they are implicated in several vesicular mechanisms as autophagy and receptor trafficking. For example, GABARAPL1 promotes cell surface expression of the Kappa Opioid Receptor (KOR) by facilitating its trafficking along the secretory pathway. Accordingly, knockdown of GABARAPL1 decreases the surface expression of KOR [19]. Binding of GABARAPL1 to the KOR is based on hydrophobic interactions, and the N-terminal region of GABARAPL1 is important for binding 
to tubulin and surface expression of the KOR [20]. In addition, the tubulin binding region is important for $\mathrm{GABA}_{\mathrm{A}}$ receptor clustering [21].

We observed that EGFR membrane expression and GABARAPL1 production are increased during hypoxia. We therefore hypothesized that GABARAPL1 mediates increased EGFR membrane-expression.

\section{Material and methods}

Cell culture Colorectal adenocarcinoma (HT29, HCT116), mammary adenocarcinoma (MCF7), glioblastoma astrocytoma (U87, U373) cells and large cell lung cancer (H460) were maintained as described by ATCC. Doxycycline-inducible $(1 \mu \mathrm{g} / \mathrm{ml})$ shRNA targeting GABARAPL1 [TTACCTTACTTCATACTTGCCC] or scrambled control [CGAGGGCGACTTAACCTTAGG] was achieved through lentiviral pTripZ (Thermo Scientific) expression as described previously [14]. Knockdown of UPR components (siRNAs) and HIF1 $\alpha$ (shRNA expression) was done as described previously [15]. Hypoxia exposure was done using a MACS VA500 workstation (Don Whitley Scientific) that allows maintaining a stable $\mathrm{O}_{2}$ concentration over time due to continuous gas influx.

Immunoblot Cells were lysed and processed as described previously [11]. Proteins were probed with antibodies against GABARAPL1 (Proteintech group, 11010-1-AP), EGFR (SC03, Santa Cruz), elF4E, p-ERK (Cell signaling) and Beta-Actin (MP Biomedicals). Bound antibodies were visualized using HRP-linked antibodies (Cell Signaling).

Membrane isolation Cells were exposed to anoxia $\left(\mathrm{O}_{2}<0.02 \%\right)$, trypsinized and resuspended in hypotonic swelling buffer $(10 \mathrm{mM}$ Tris, $1 \mathrm{mAmprcaptoethanol,} 100 \mu \mathrm{m}$ EDTA and HEPES, pH 7,3) for $1 \mathrm{~h}$ on ice. Cell ghosts were ultracentrifuged at $145000 \mathrm{xg}$ for 30 minutes and analysed by western blot.

Clinical study GABARAPL1 mRNA expression was determined by quantitative PCR in 86 head and neck cancer patients [22]) and used to median-dichotomize the patient cohort. Differences between patient characteristics (Suppl table1) were assessed with a $\chi^{2}$-test or student's t-test. Differences in recurrence-free survival were determined using Kaplan-Meier survival analysis and Log-Rank (Mantel-Cox) test.

Immunofluorescence Cells were fixed with 4\% paraformaldehyde, permeabilized, blocked and probed with anti-GABARAPL1 (Aviva Systems Biology, ARP55398_P050) and anti-EGFR (Oregon Green-labeled Cetuximab). Anti-GABARAPL1 was visualized using goat anti-rabbit Alexa-555 (Invitrogen, A-21428).

Quantitative real-time PCR mRNA was isolated with NucleoSpin RNA II kit (Bioke) and reverse transcribed using I-script (BIO-RAD). Expression was normalized to 18S. Primers used are listed in Supplementary table 2. 
Flow cytometry single cells were labelled with anti-EGFR (Cetuximab-oregon green) or anti-CA9-hilyte-488(Novus Biologicals) and anti-GLUT1-APC(BD biosciences) Membrane expression was determined by flow cytometry..

Click-it chemistry: After 16 hours of anoxia exposure, azide-labeled methionine (L-AHA) was allowed to incorporate for 8 hours. Subsequently, cells were trypsinised and labelled by click-it chemistry according to the manual (Thermo Fisher) and analysed by flow cytometry.

Statistics Data were analysed using GraphPad Prism. Student's $t$ test was used for single comparisons. Multiple testing was done using a repeated measures ANOVA with a Bonferroni post-hoc test. $P$-values $<0.05$ were considered statistically significant.

\section{Results}

GABARAPL1 expression is increased during hypoxia

Previously, microarray analysis on HT29, MCF7 and DU145 cancer cell lines during hypoxia $\left(\mathrm{O}_{2}<0.02 \%\right)$ were performed [23]. In all three cell lines, hypoxia increased GABARAPL1 mRNA expression (Suppl. Fig. 1A). These results were validated on independent experiments by qPCR analysis of HT29, MCF7, DU145, U87 (Fig. 1A), U373 (fig1B) and HCT116 (fig1C) cells. GABARAPL1 induction is dependent on severity of hypoxia as moderate hypoxia $\left(0.2 \% \mathrm{O}_{2}\right)$ is less capable of inducing GABARAPL1 expression in HT29 and U373 cells (Suppl. Fig 1B) after 24 hours exposure. In line with RNA expression, GABARAPL1 protein expression levels are induced during anoxia $\left(\mathrm{O}_{2}<0.02 \%\right)$ in U87 and HT29 cells, but not in U373 (Fig. 1D).

Cells adapt to hypoxia through activation of multiple pathways, including HIF and UPR signalling. We therefore investigated the contribution of these pathways to hypoxia-induced GABARAPL1 expression. Knockdown of HIF1 a did not change the extent of GABARAPL1 upregulation in HT29 and U373 cells during hypoxia (Fig. 1C). Next, we investigated the three parallel arms of the UPR; PERK, ATF6 and IRE1. Knockdown of PERK, but not ATF6 or IRE1 abrogated hypoxia-induced GABARAPL1 mRNA expression (Fig. 1D). Downstream of PERK, targeting ATF4 or CHOP revealed that ATF4 is required for GABARAPL1 mRNA upregulation during hypoxia. Together, this indicates that transcriptional upregulation of GABARAPL1 during hypoxia is mediated by PERK/ATF4 signalling. 
A

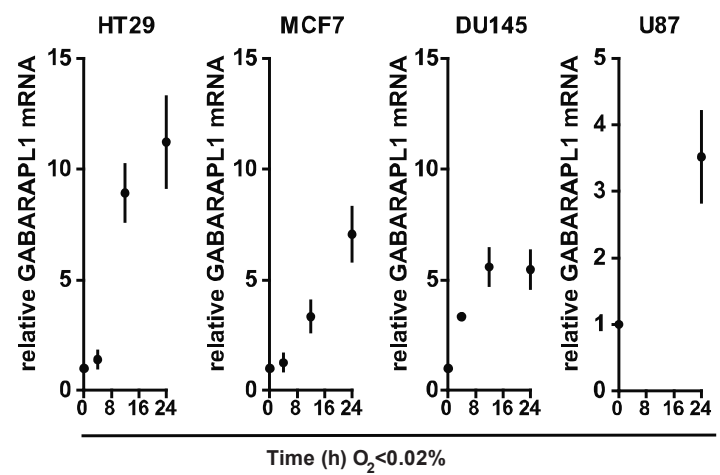

C

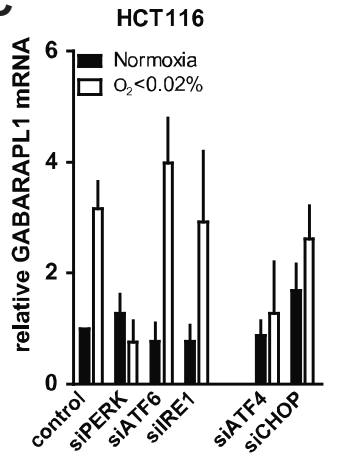

U373

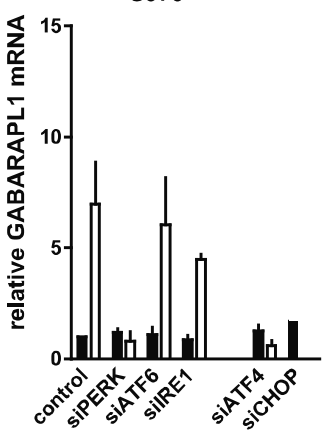

B
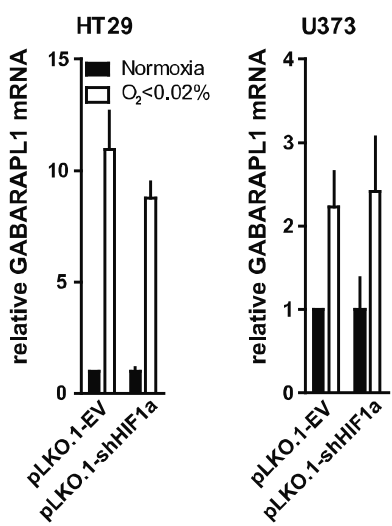

D

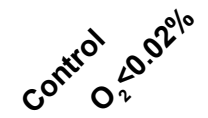

HT29

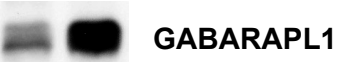

U87

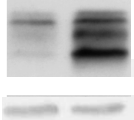

$\beta$-actin

GABARAPL1

\section{$\beta$-actin}

GABARAPL1

\section{$\beta$-actin}

Figure 1. Increased GABARAPL1 expression is PERK/ATF4 dependent.

(A) Transcriptional regulation of GABARAPL1 in HT29, MCF7, Du145 and U87 cancer cells during anoxia $\mathrm{O}_{2}$ $<0.02 \%$ ). (B) HIF1 $\alpha$ knockdown (shHIF1 $\alpha$ ) in HT2 9 and U3 73 cells does not affect GABARAPL1 mRNA expression. (C) Transcriptional upregulation of GABARAPL1 during hypoxia is dependent on PERK/ATF4 signaling. A-C, $\mathrm{n}=3$, mean \pm SEM. (D) GABARAPL1 protein expression in induced during anoxia $\left(\mathrm{O}_{2}<0.02 \%\right)$

GABARAPL1 mRNA expression correlates with tumour hypoxia and poor clinical outcome

As GABARAPL1 is upregulated during hypoxia, we assessed whether GABARAPL1 mRNA expression could serve as surrogate marker for tumour hypoxia. In a panel of 14 primary human head and neck squamous cell carcinoma (HNSCC) xenografts [24], GABARAPL1 mRNA expression and hypoxia (pimonidazole) were determined. The hypoxic fraction [25] correlated with GABARAPL1 mRNA expression (Fig. 2A; Pearson $r=0.63 ; p<0.05$ ), suggesting that GABARAPL1 mRNA can be used as a surrogate marker for tumour hy- 
poxia in HNSCC. In agreement with the PERK/ATF4-dependence, GABARAPL1 mRNA expression correlates with another UPR-dependent hypoxia regulated gene, LC3b [15](Fig. 2B; Pearson r=0.54; $p<0.05$ ).

A

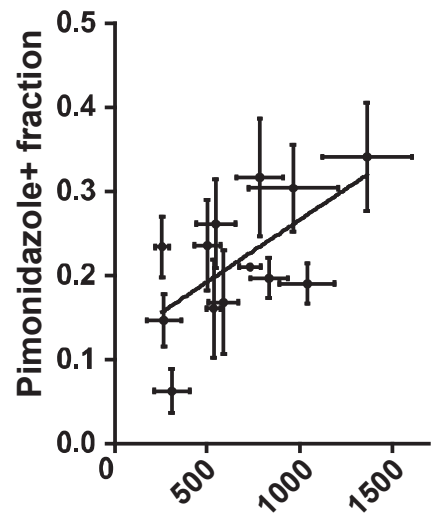

GABARAPL1 mRNA

C



B

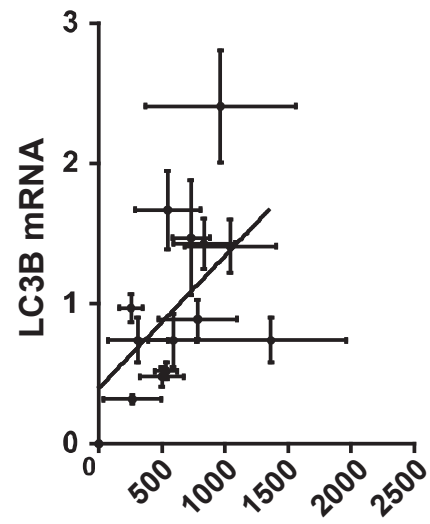

GABARAPL1 mRNA

D

Locoregional Control

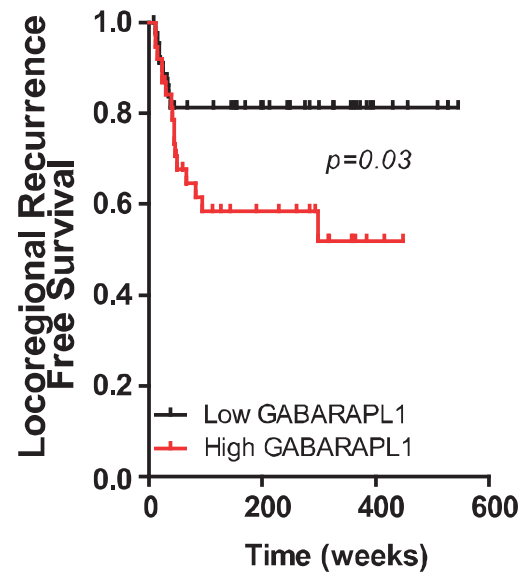

Figure 2. GABARAPL1 mRNA expression correlates with tumour hypoxia and poor clinical outcome.

(A) In 14 HNSCC xenografts, GABARAPL1 mRNA expression was determined and correlated with (hypoxic fraction) (pimonidazole) and (B) LC3b mRNA. (C) high GABARAPL1 mRNA expression is associated with increased local $(p=0.10)$ and (D) locoregional recurrences $(p=0.03)$ in a cohort of 86 HNSCC patients. 
Tumour hypoxia is a negative prognostic and predictive factor of patient outcome as it decreases the efficacy of radio- and chemotherapy. This has been particularly well established in HNSCC $[26,27]$.

We therefore questioned if GABARAPL1 expression has prognostic value in a cohort of 86 HNSCC patients after chemoradiation [22]. Local $(p=0.19)$ and locoregional $(p=0.038)$ control was worse in patients with high GABARAPL1 expressing tumours (Fig. 2C and 2D)

\section{GABARAPL1 is required for increased EGFR membrane expression during hypoxia}

Recent reports indicate that EGFR expression is increased during hypoxia [17]. To investigate if GABARAPL1 mediates EGFR membrane expression, cellular localization was assessed using confocal microscopy. In U87 cells, GABARAPL1 is present in a typical vesicle-like pattern. During anoxia, GABARAPL1 is also observed at the cellular perimeter and colocalizes with EGFR at the plasma membrane (fig 3A). In accordance, both EGFR and GABARAPL1 are enriched in the membrane fraction of anoxia exposed HT29 cells (Fig. 3B).

To determine if GABARAPL1 is involved EGFR membrane-expression, doxycyclineinducible GABARAPL1 knockdown cells were engineered (Fig 3C). EGFR expression at the plasma membrane of control and GABARAPL1 knockdown in HT29, U373, MDAMB231 and U87 cells after exposure to hypoxia $\left(\mathrm{O}_{2} 0.2 \%\right)$ and anoxia $\left(\mathrm{O}_{2}<0.02 \%\right)$ was determined by flow cytometry.

EGFR membrane expression is induced in all control (SCR) cell lines during hypoxia and anoxia (Fig 3D). This increased EGFR membrane expression was inhibited after GABARAPL1 knockdown (Fig 3D)

To determine if the reduced EGFR membrane expression is the result of a reduced total EGFR expression, EGFR mRNA and total protein expression was determined. During hypoxic and anoxic conditions, EGFR mRNA and total protein levels are not altered or even increased in GABARAPL1 knockdown cells (Fig 3F). Notably, EGFR mRNA of U373 and MDA-MB-231 GABARAPL1 knockdown cells during normoxic conditions was increased (FIG 3E).

To determine if GABARAPL1 is required uniquely for EGFR trafficking during hypoxia, overall membrane protein expression in SCR control and GABARAPL1 knockdown cells was determined using click-it chemistry. GABARAPL1 knockdown reduces membrane protein expression in both HT29 (23\%) and MCF7 (43\%), indicating a role in protein trafficking broader than EGFR (Fig 3G). In line, CA9 plasma membrane expression was decreased after GABARAPL1 knockdown in 4 cell lines tested (Suppl fig 2A). Reduced CA9 membrane expression was not caused by reduced production in GABARAPL1 knockdown cells as indicated by total protein expression (Fig 3F) and qPCR (Suppl. Fig 2C). Interestingly, in contrast to EGFR and CA9, GLUT1 membrane expression is not affected by GABARAPL1 knockdown, despite increased CA9 and GLUT mRNA expression in MDA-MD-231 GABARAPL1 knockdown cells (Suppl Fig 2C and 2D). 


\section{A}

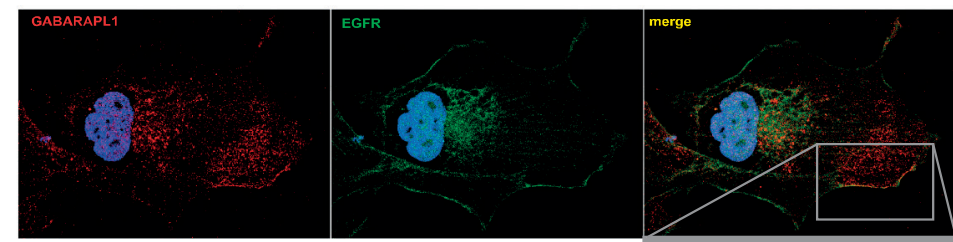

B
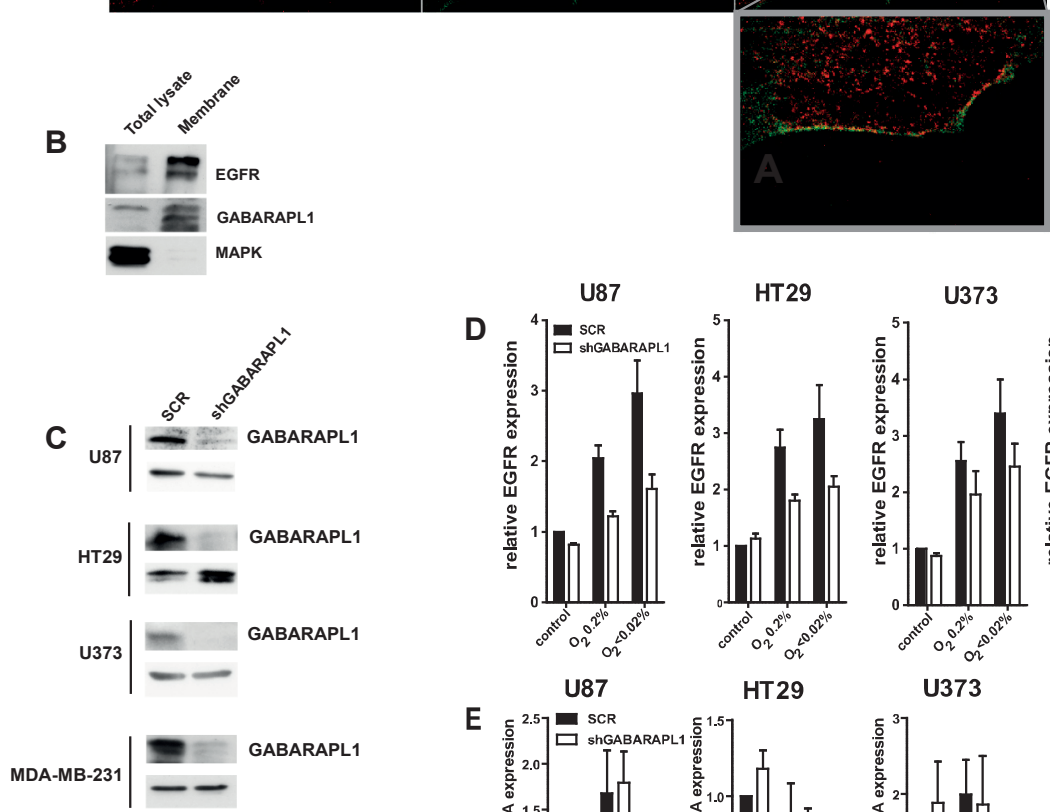

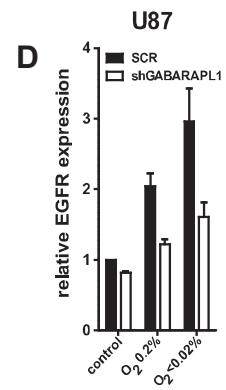

U87
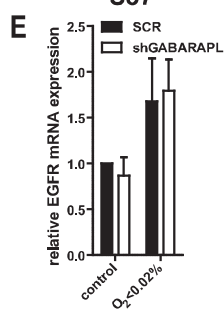

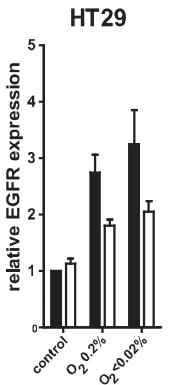

HT 29

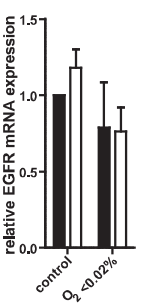

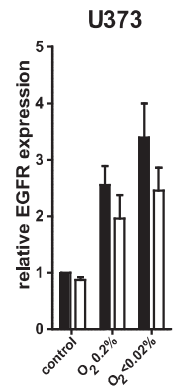

U373

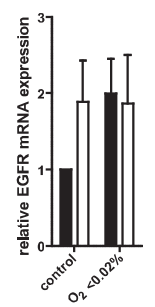

MDA-MB-231

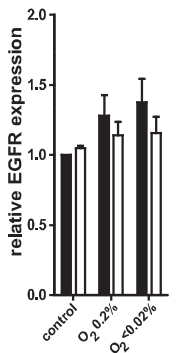

MDA-MB-231

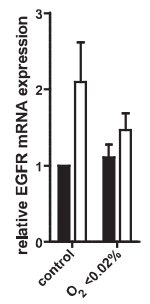

G

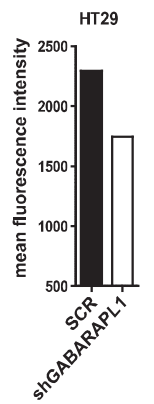

$\mathbf{F}$

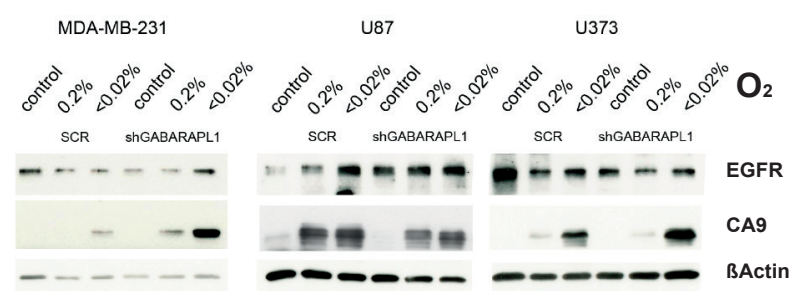

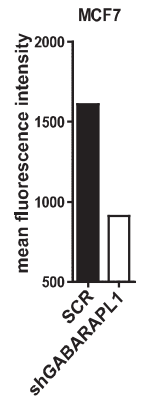

Figure 3. GABARAPL1 is required for increased EGFR membrane expression during hypoxia.

(A) Immunofluoresent staining of GABARAPL1(red) and EGFR(green) in U87 cells. (B) immunoblot of total and membrane fraction of anoxic HT29 cells. (C) GABARAPL1 knockdown cell lines. (D) FACS analysis of EGFR membrane expression during hypoxic $\left(\mathrm{O}_{2} 0.2 \%\right)$, and anoxic $\left(\mathrm{O}_{2}<0.02 \%\right)$, conditions $n=4$ mean \pm SEM (E) EGFR mRNA expression. $n=3$ mean \pm SEM (F) Immunoblot of total EGFR and CA9 levels (G) Membrane expression of proteins assessed by Click-it chemistry. 


\section{Discussion}

EGFR expression and tumour hypoxia are negative prognostic factors associated with poor treatment outcome. In this study we explored the role of GABARAPL1, which we identified as a hypoxia induced protein required for the trafficking of EGFR to the plasma membrane during hypoxia.

Here we show that the GABARAP family member GABARAPL1 is induced during hypoxia on both mRNA and protein levels. Interestingly, other GABARAP family members, GABARAP and GABARAPL2, are not induced during hypoxia, suggesting a differential role for gabarapl1 during these conditions (data not shown). Several proteins, including EGFR can be upregulated during hypoxia [17] and require transportation to the membrane. Since the GABARAP family is implicated in several processes like anterograde trafficking of several receptors like the Kappa Opioid Receptor [19], angiotensin [28, 29] and $G_{A B A}$ receptor[30] we questioned the role of GABARAPL1 in EGFR trafficking. EGFR and other growth factor receptors are often amplified or mutated in cancer and may promote hypoxia tolerance $[16,31]$. In this report we show that the membrane expression of EGFR is elevated during hypoxia and that this effect is GABARAPL1 dependent. It is therefore tempting to speculate that the observed relation between high GABARAPL1 and outcome is partly mediated through facilitating enhanced EGFRsignalling.

Hypoxia induced membrane expression of EGFR in most of the cell lines tested. Although consistent, this was less pronounced in the parental cell lines compared to the genetically modified cell lines (Suppl Fig 3). In the PTRIPZ lentiviral system, parallel to shRNA expression, red fluorescent protein is expressed. This may lead to increased misfolding of proteins and/or ROS formation and may contribute to increased EGFR upregulation during hypoxia in the transgenic cells.

For efficient membrane expression and further downstream signalling, clustering of receptors, including EGFR is essential. Chen et al showed that the tubulin-binding motif of GABARAP is required for clustering of the $\mathrm{GABA}_{A}$ receptor [32]. Also GABARAPL1 contains this binding motif, which is located in the first 22 amino acids [33]. GABARAPL1 acts as a linker between microtubules and the KOR to facilitate anterograde trafficking [34]. GABARAPL1 may act similarly in EGFR receptor clustering and/ or trafficking.

Together our data support the specific expression and/or translocation of proteins during hypoxia that allow cells to adapt to conditions of low oxygenation. GABARAPL1 in this respect seems to fulfil a specific role in trafficking of EGFR and CA9. 


\section{Acknowledgements}

This work was financially supported by the Dutch Cancer Society (KWF Grants UM 20104714 and 2012-5506 to K.R.), STOPhersentumoren.nl and zeldzame ziektenfonds (to K.R.). 


\section{References}

1. Lartigau, E., et al., Variations in tumour oxygen tension (pO2) during accelerated radiotherapy of head and neck carcinoma. Eur J Cancer, 1998. 34(6): p. 856-61.

2. Nordsmark, M., et al., Prognostic value of tumor oxygenation in 397 head and neck tumors after primary radiation therapy. An international multi-center study. Radiotherapy and oncology : journal of the European Society for Therapeutic Radiology and Oncology, 2005. 77(1): p. 18-24.

3. Pennacchietti, S., et al., Hypoxia promotes invasive growth by transcriptional activation of the met protooncogene. Cancer Cell, 2003. 3(4): p. 347-61.

4. Chang, Q., et al., Hypoxia predicts aggressive growth and spontaneous metastasis formation from orthotopically grown primary xenografts of human pancreatic cancer. Cancer Res, 2011. 71(8): p. 311020.

5. Rademakers, S.E., et al., Pattern of CAIX expression is prognostic for outcome and predicts response to ARCON in patients with laryngeal cancer treated in a phase III randomized trial. Radiother Oncol, 2013. 108(3): p. 517-22.

6. Hirota, K. and G.L. Semenza, Regulation of angiogenesis by hypoxia-inducible factor 1. Crit Rev Oncol Hematol, 2006. 59(1): p. 15-26.

7. Svastova, E., et al., Hypoxia activates the capacity of tumor-associated carbonic anhydrase IX to acidify extracellular pH. FEBS letters, 2004. 577(3): p. 439-45.

8. Koritzinsky, M., et al., Two phases of disulfide bond formation have differing requirements for oxygen. J Cell Biol, 2013. 203(4): p. 615-27.

9. Lu, P.D., H.P. Harding, and D. Ron, Translation reinitiation at alternative open reading frames regulates gene expression in an integrated stress response. J Cell Biol, 2004. 167(1): p. 27-33.

10. Kojima, E., et al., The function of GADD34 is a recovery from a shutoff of protein synthesis induced by ER stress: elucidation by GADD34-deficient mice. Faseb J, 2003. 17(11): p. 1573-5.

11. Koritzinsky, M., et al., Phosphorylation of elF2alpha is required for mRNA translation inhibition and survival during moderate hypoxia. Radiother Oncol, 2007. 83(3): p. 353-61.

12. Walter, P. and D. Ron, The unfolded protein response: from stress pathway to homeostatic regulation. Science, 2011. 334(6059): p. 1081-6.

13. Rouschop, K.M., et al., PERK/elF2alpha signaling protects therapy resistant hypoxic cells through induction of glutathione synthesis and protection against ROS. Proc Natl Acad Sci U S A, 2013. 110(12): p. 4622-7.

14. Schaaf, M.B., et al., The autophagy associated gene, ULK1, promotes tolerance to chronic and acute hypoxia. Radiotherapy and oncology : journal of the European Society for Therapeutic Radiology and Oncology, 2013. 108(3): p. 529-34.

15. Rouschop, K.M., et al., The unfolded protein response protects human tumor cells during hypoxia through regulation of the autophagy genes MAP1LC3B and ATG5. J Clin Invest, 2010. 120(1): p. 127-41.

16. Theys, J., et al., The deletion mutant EGFRvIll significantly contributes to stress resistance typical for the tumour microenvironment. Radiotherapy and oncology : journal of the European Society for Therapeutic Radiology and Oncology, 2009. 92(3): p. 399-404.

17. Franovic, A., et al., Translational up-regulation of the EGFR by tumor hypoxia provides a nonmutational explanation for its overexpression in human cancer. Proceedings of the National Academy of Sciences of the United States of America, 2007. 104(32): p. 13092-7.

18. Nijkamp, M.M., et al., Interaction of EGFR with the tumour microenvironment: implications for radiation treatment. Radiotherapy and oncology : journal of the European Society for Therapeutic Radiology and Oncology, 2013. 108(1): p. 17-23.

19. Chen, C., et al., Effects of C-terminal modifications of GEC1 protein and gamma-aminobutyric acid type $A$ $(G A B A(A))$ receptor-associated protein (GABARAP), two microtubule-associated proteins, on kappa opioid receptor expression. J Biol Chem, 2011. 286(17): p. 15106-15. 
20. Chen, C., et al., GEC1 interacts with the kappa opioid receptor and enhances expression of the receptor. The Journal of biological chemistry, 2006. 281(12): p. 7983-93.

21. Wang, H. and R.W. Olsen, Binding of the GABA(A) receptor-associated protein (GABARAP) to microtubules and microfilaments suggests involvement of the cytoskeleton in GABARAPGABA(A) receptor interaction. Journal of neurochemistry, 2000. 75(2): p. 644-55.

22. Pramana, J., et al., Gene expression profiling to predict outcome after chemoradiation in head and neck cancer. Int J Radiat Oncol Biol Phys, 2007. 69(5): p. 1544-52.

23. Starmans, M.H., et al., The prognostic value of temporal in vitro and in vivo derived hypoxia geneexpression signatures in breast cancer. Radiother Oncol, 2012. 102(3): p. 436-43.

24. Stegeman, H., et al., Predictive value of hypoxia, proliferation and tyrosine kinase receptors for EGFRinhibition and radiotherapy sensitivity in head and neck cancer models. Radiother Oncol, 2013. 106(3): p. 383-9.

25. Hoeben, B.A., et al., Systematic analysis of 18F-FDG PET and metabolism, proliferation and hypoxia markers for classification of head and neck tumors. BMC cancer, 2014. 14: p. 130.

26. Brizel, D.M., et al., Oxygenation of head and neck cancer: changes during radiotherapy and impact on treatment outcome. Radiother Oncol, 1999. 53(2): p. 113-7.

27. Nordsmark, M., et al., Prognostic value of tumor oxygenation in 397 head and neck tumors after primary radiation therapy. An international multi-center study. Radiother Oncol, 2005. 77(1): p. 18-24.

28. Alam, J., et al., C-terminal processing of GABARAP is not required for trafficking of the angiotensin II type 1 A receptor. Regul Pept, 2010. 159(1-3): p. 78-86.

29. Cook, J.L., et al., The trafficking protein GABARAP binds to and enhances plasma membrane expression and function of the angiotensin I/ type 1 receptor. Circ Res, 2008. 102(12): p. 1539-47.

30. Chen, Z.W., et al., C-terminal modification is required for GABARAP-mediated GABA(A) receptor trafficking. J Neurosci, 2007. 27(25): p. 6655-63.

31. Weppler, S.A., et al., Expression of EGFR variant vIII promotes both radiation resistance and hypoxia tolerance. Radiotherapy and oncology : journal of the European Society for Therapeutic Radiology and Oncology, 2007. 83(3): p. 333-9.

32. Chen, L., et al., The gamma-aminobutyric acid type A (GABAA) receptor-associated protein (GABARAP) promotes GABAA receptor clustering and modulates the channel kinetics. Proc Natl Acad Sci U S A, 2000. 97(21): p. 11557-62.

33. Mansuy, V., et al., GEC1, a protein related to GABARAP, interacts with tubulin and GABA(A) receptor. Biochemical and biophysical research communications, 2004. 325(2): p. 639-48.

34. Chen, Y., et al., GEC1-kappa opioid receptor binding involves hydrophobic interactions: GEC1 has chaperone-like effect. The Journal of biological chemistry, 2009. 284(3): p. 1673-85. 


\section{Supplementary figures}
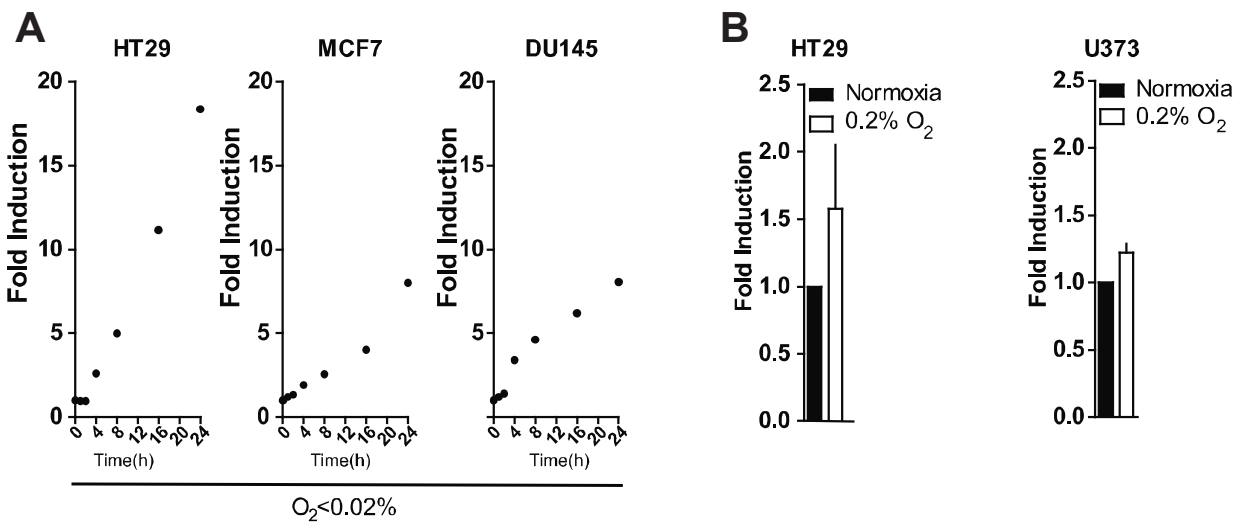

\section{Supplementary Figure 1}

A) Micro array analysis shows an increased GABARAPL1 expression during hypoxia $\left(\mathrm{O}_{2}<0.02 \%\right)$

B) GABARAPL1 mRNA expression during hypoxia $\left(\mathrm{O}_{2} 0.2 \%\right)$ as assessed by qPCR $n=3$ mean $\pm \mathrm{SEM}$ 
A

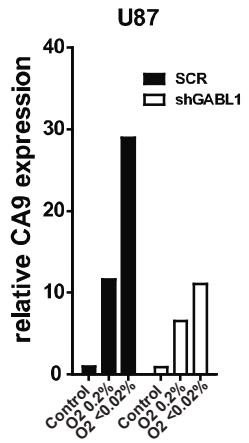

B

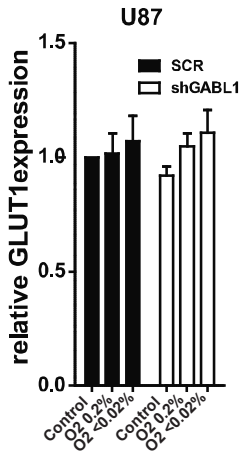

C

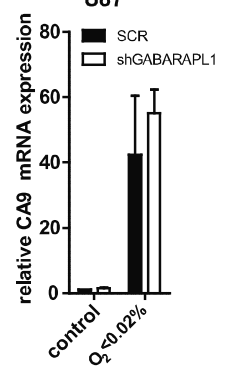

U87

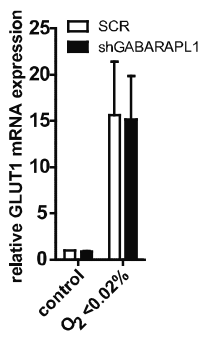

HT29

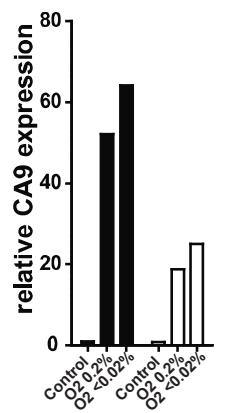

HT29

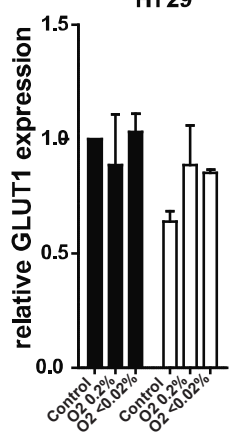

HT 29

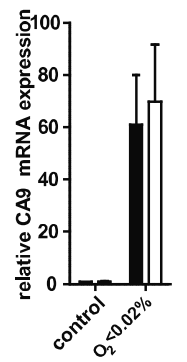

HT 29

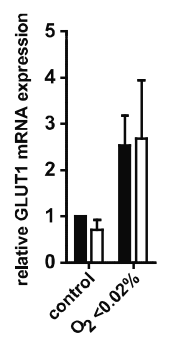

U373

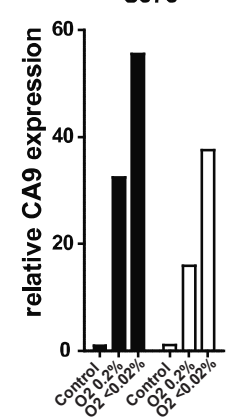

U373

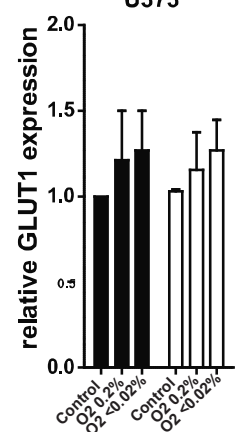

U373

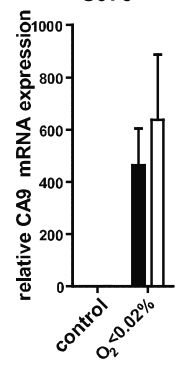

U373

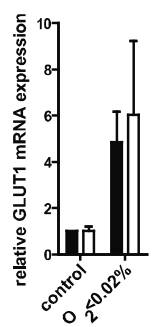

MDA-MB-231
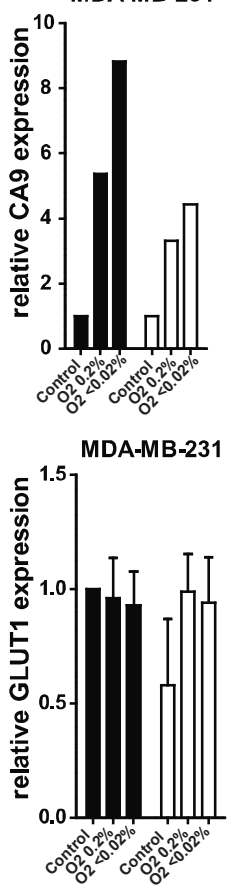

MDA-MB-231

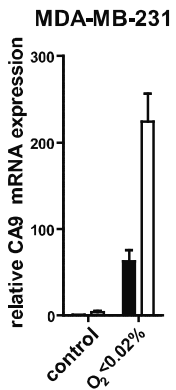

MDA-MB-231

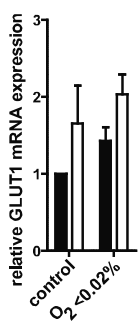

\section{Supplementary figure 2}

(A B) Membrane expression levels of CA9(A) and GLUT1(B) as determined by FACS in GABARAPL1 knockdown cells during hypoxia $\left(0.2 \% \mathrm{O}_{2}\right)$ and anoxia $\left(<0.02 \% \mathrm{O}_{2}\right)$. Shown graph is a representative example $n=3$. B $n=3$ mean \pm SEM (C D) relative mRNA expression of CA9(C) and GLUT1(D) in GABARAPL1 knockdown cells during anoxia( $<0.02 \%$ O2) $n=4$ mean \pm SEM 


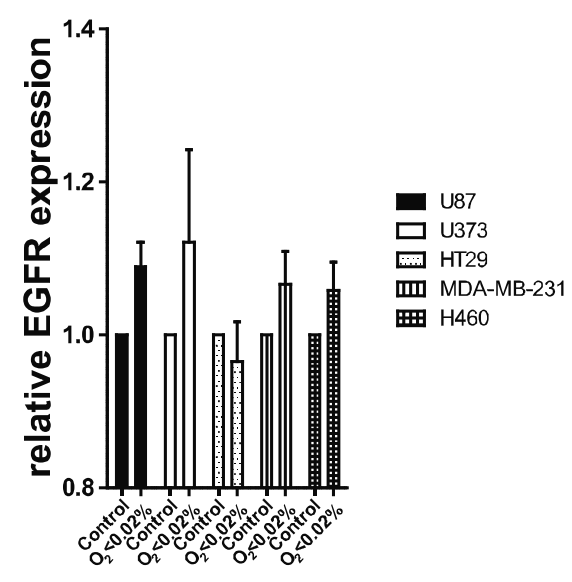

\section{Supplementary figure 3}

Relative EGFR expression in parental cell lines as determined by FACS $n=3$ mean \pm SEM 


\section{Chapter}

\section{EGFRvIII expression triggers a metabolic dependency and therapeutic vulnerability sensitive to autophagy inhibition}

Barry Jutten ${ }^{1}$, Tom GH Keulers ${ }^{1}$, Hanneke JM Peeters ${ }^{1}$, Marco BE Schaaf ${ }^{1}$, Kim GM Savelkouls ${ }^{1}$, Inge Compter ${ }^{1}$, Ruud Clarijs², Olaf EMG Schijns ${ }^{3}$, Linda Ackermans ${ }^{3}$, Onno PM Teernstra ${ }^{3}$, Marijke I Zonneveld ${ }^{1}$, Resi ME Colaris ${ }^{2}$, Ludwig Dubois ${ }^{1}$, Marc A Vooijs ${ }^{1}$, Johan Bussink ${ }^{4}$, Julio Sotelo ${ }^{5}$, Jan Theys ${ }^{1}$, Guido Lammering ${ }^{1,6}$ and Kasper MA Rouschop ${ }^{1}$

${ }^{1}$ Maastricht Radiation Oncology (MaastRO) lab, GROW - School for Oncology and Developmental Biology, Maastricht University, Maastricht, the Netherlands

${ }^{2}$ Department of Clincial Pathology, Zuyderland MC, Sittard-Geleen, The Netherlands ${ }^{3}$ Department of Neurosurgery, Maastricht University Medical Centre ${ }^{4}$ Department of Radiation Oncology, Radboud University Medical Center, Nijmegen, The Netherlands

${ }^{5}$ Neuroimmunology and Neuro-Oncology Unit, National Institute of Neurology and Neurosurgery, Mexico City, Mexico ${ }^{6}$ Heinrich- Heine University Duesseldorf, Germany 


\section{Abstract}

Expression of EGFRvIII is frequently observed in glioblastoma and is associated with increased cellular proliferation, enhanced tolerance to metabolic stresses, accelerated tumour growth, therapy resistance and poor prognosis. We observed that expression of EGFRvIII elevates the activation of macroautophagy/autophagy during starvation and hypoxia and explored the underlying mechanism and consequence. Autophagy was inhibited (genetically or pharmacologically) and its consequence for tolerance to metabolic stress and its therapeutic potential in (EGFRvIII+) glioblastoma was assessed in cellular systems, (patient derived) tumour xenografts and glioblastoma patients. Autophagy inhibition abrogated the enhanced proliferation and survival advantage of EGFRvIII+ cells during stress conditions, decreased tumour hypoxia and delayed tumour growth in EGFRvIII+ tumours. These effects can be attributed to the supporting role of autophagy in meeting the high metabolic demand of EGFRvIII cells. As hypoxic tumour cells greatly contribute to therapy resistance, autophagy inhibition revokes the radioresistant phenotype of EGFRvIII+ tumours in (patient derived) xenograft tumours. In line with these findings, retrospective analysis of glioblastoma patients indicated that chloroquine treatment improves survival of all glioblastoma patients, but patients with EG$\mathrm{FRvIII}^{+}$glioblastoma benefited most. Our findings disclose the unique autophagy dependency of EGFRvIII+ glioblastoma as a therapeutic opportunity. Chloroquine treatment may therefore be considered as an additional treatment strategy for glioblastoma patients and can reverse the worse prognosis of patients with EGFRvIII ${ }^{+}$glioblastoma. 


\section{Introduction}

EGFR (epidermal growth factor receptor) is a transmembrane glycoprotein and 1 of 4 members of the ERBB family of tyrosine kinase receptors. Binding of one of its natural ligands results in homo- or heterodimerization of the receptor, autophosphorylation and subsequent activation of signal transduction pathways. These pathways are involved in regulating cellular proliferation, differentiation, and survival.(1,2) Although present in normal cells, EGFR is overexpressed in a variety of tumours and has been associated with poor prognosis and decreased survival (3). EGFR activation also plays a role in resistance to chemotherapy and radiation treatment in tumour cells $(4,5)$.

In cancer, EGFR is often mutated, leading to enhanced or sustained receptor signaling. One of the most common variants of EGFR is an exon 2-7 deletion mutant, EGFRvIII (also termed $\triangle E G F R$ or de2-7EGFR) (6-9). This deletion results in a truncated receptor that lacks 267 amino acids in the extracellular binding domain. This deletion leads to important functional changes; the receptor is unable to bind its ligands, but becomes constitutively active, resulting in uncontrolled pro-oncogenic effects and thereby promotes neoplastic transformation and tumourigenicity (10-13). EGFRvIII expression is observed in several malignancies, including glioblastoma (GBM), non-small lung cell carcinoma, breast cancer, prostate cancer and head and neck cancer $(6,8,9)$ and is associated with poor prognosis (14). In GBM patients with amplification of the EGFR gene, the overall prevalence of EGFRvIII is 50-60\% (15) and has been reported to contribute to tumour stem cell maintenance (16-18). Expression of EGFRvIII greatly enhances GBM tumourigenicity in vivo $(19,20)$ and stimulates cell invasion in vitro and in vivo $(21,22)$.

The tumour microenvironment is characterized by extreme heterogeneities in nutrient supply and oxygenation that arise primarily due to a poorly developed and/or functioning vascular network. Gradients of oxygenation exist around individual perfused vessels and range from normal values $\left(\sim 5 \% \mathrm{O}_{2}\right)$ near the vessel wall to anoxia in perinecrotic regions. Transient changes in blood flow also lead to strong temporal changes in oxygenation within specific tumour regions (23). The percentage of viable hypoxic tumour cells within individual tumours with otherwise similar clinical features varies tremendously among patients (24) and is clinically important because high levels of tumour hypoxia correlate with poor prognosis and a more aggressive phenotype (2426). The source of the variability in hypoxia among different tumours is likely due to the acquisition of changes that drive increased hypoxia tolerance (27). Strikingly, EGFRvIII expression in vivo is rapidly lost when tumour cells are cultured in vitro under nutrientrich conditions (28). This observation suggests that in the tumour microenvironment, which is characterized by heterogeneities in nutrient supply and oxygenation, EGFRvIIIexpressing tumour cells have a survival advantage, a finding supported by pre-clinical evidence $(12,13)$.

To survive starvation and hypoxia, cells respond by upregulation of autophagy (Greek for "self-eating") (29,30). A number of recent studies have demonstrated that 
human tumour cell lines induce autophagy when exposed in vitro to hypoxia and/or metabolic stress, (31-33) which mediates both selective and bulk degradation of proteins, cytoplasmic content, and organelles, and enables a cell to recycle constituents and provide itself with the necessary nutrients to maintain energy levels, protein synthesis and essential metabolic processes (34).

Based on our observations that EGFRvIll expression results in increased autophagy activation in metabolically challenged cells, we hypothesized that the increased survival and growth of EGFRvIll-expressing cells and tumours during stressful conditions is supported by autophagy. Targeting autophagy could therefore be a potential tool to lower tolerance to metabolic stresses and increase cell killing of EGFRvIll-expressing GBM cells.

\section{Materials and Methods}

\section{In vivo experiments}

Animal facilities and experiments were in accordance with local institutional guidelines and approved by the local animal welfare committee. Experiments were performed as described previously (33). Briefly, tumours were grown in NMRI-nu (nu/nu) female mice (Charles River). Cells in matrigel (BD Biosciences, 354234) were injected subcutaneously $\left(1.5 \times 10^{6}\right.$ cells). For the generation of primary human xenografts, a small piece of biopsy (approx. $1 \mathrm{~mm}^{3}$ ) was implanted for the generation of the first generation tumour (growth until $1 \mathrm{~cm}^{3}$ ). This tumour was cut into multiple pieces, implanted in recipient mice and experiments were subsequently performed on these $2^{\text {nd }}$ generation tumours. Tumour size was assessed by caliper measurement in 3 orthogonal diameters. Mice treated with CQ (Sigma Aldrich, C6628) received $60 \mathrm{mg} / \mathrm{kg}$ CQ for 7 consecutive days. For radiation treatment, tumours were positioned in the irradiation field using a custom-built jig and irradiated with a single dose of $10 \mathrm{~Gy}$ (15 MeV e-) using a linear accelerator (Varian, Palo Alto, CA, USA)(35) Number of animals used are listed in the respective figure legend.

\section{Patient studies}

The retrospective analyses and the establishment of patient derived xenografts were approved by the respective local medical ethics committees. All patients with a primary GBM, treated in Maastricht with radiotherapy between 2007 and 2012 with sufficient histological tissue available and who had completed the planned treatment with temozolomide $\left(75 \mathrm{mg} / \mathrm{m}^{2} /\right.$ day) and radiotherapy (60Gy), were included in the analysis (Fig. $6 B$ and table 1). The effect of $C Q$ on prognosis in EGFRvIll-expressing GBM was evaluated on 43 GBM patients (of which sufficient tumour tissue was still available) that were included in a randomized, placebo controlled phase II trial (Fig. 6C and table 2) (36). 


\section{Reagents and cell lines}

Unless specified otherwise, all reagents were obtained from Sigma Chemical Co. (the Netherlands) and all electrophoresis reagents were from Bio-Rad (the Netherlands). MEM- $\alpha$ medium was obtained from Invitrogen (10370070). Glutamax-I (35050038) and pyruvate (11360039) were obtained from Life Technologies, DMEM (BE12-604F) and fetal bovine serum (FBS) were from GE-Healthcare. Glucose determination kits were purchased from Biosentec (010). Cell lines were obtained from and maintained as described by ATCC (HTB-14 (U87) and HTB-17 (U373)), negative for mycoplasma contamination (Lonza Mycoalerts, LT07-318). The cells' origin was authenticated by STR analysis (Identicell). Cell lines that endogenously express EGFRvIII (DKMG and BS153) were kindly provided by Malte Kriegs and Nina Struve (Hamburg, Germany) and maintained as described previously (37). For doxycycline (Sigma, D9891) inducible knockdown of Lc3b, shRNA [TTTCTCACTCTCATACACCTCT] (Sigma Aldrich, TRCN0000152696) was expressed from Tet-pLKO-puro (addgene, 21915; depositor Dmitri Wiederschain (38)) as described previously (39). ATG7 knockdown was induced using PTRIPZ lentiviral delivery of shATG7 [ATACAGTGTTCCAATAGCTGGG] (Dharmacon). Dominant negative ULK1 ${ }^{\mathrm{K} 461}$ was used as described previously (40). Differences in autophagic flux were confirmed using mCherry-EGFP-LC3 expression from a pBabe-puro vector (addgene, 22418; depositor J. Debnath) (41).

\section{Western blotting}

Cells were lysed and processed as described previously (7) and proteins were separated through SDS-PAGE. After transfer, proteins were probed with antibodies against EGFR, EGFRvIII runs at lower height (Santa Cruz Biotechnology, sc-03), MAP1LC3B (Cell Signaling Technology, 2775S), ACTB/actin (MP Biomedicals, 08961001), p-EGFRvIII (Cell Signaling Technology, 3777), p-MAPK/ERK (Cell Signaling, 4370) or tubulin (Cell Signaling Technology, 2146). Bound antibodies were visualized using HRP-linked anti-rabbit (Cell Signaling Technology, 7074S) or anti-mouse (Cell Signaling Technology, 7076S) antibodies.

\section{Immunofluorescence and immunohistochemistry}

For immunofluorescence, cells were grown on glass coverslips (VWR, 470019-016). After exposure, cells were washed and fixed in $4 \%$ paraformaldehyde (Sigma Aldrich, p6148). After permeabilization, cells were probed with anti-MAP1LC3B (Cell Signaling Technology, 2775S) followed by anti-rabbit Alexa Fluor 488 (Invitrogen, A11006). For EGFRvIII expression determination in tumours, formalin-fixed paraffin-embedded tissue slides were probed with antibodies directed against EGFRvIII (clone L8A4, kindly provided by Dr. Bigner [Duke University Medical Center, Durham, NC]). 
For assessment of microenvironmental changes in the xenograft studies, frozen, acetone-fixed sections were stained by using anti-pimonidazole (Hypoxyprobe Inc, HP4100kit), 9F1 (rat monoclonal antibody to mouse endothelium, Department of Pathology, Radboud University Medical Center) or anti-BrdU (Sigma-Aldrich, B8434; as described previously (33)). For quantitative analysis, the slides were scanned with a computerized digital image processing system by using a high-resolution intensified solid-state camera on a fluorescence microscope (Axioskop; Zeiss, the Netherlands) with a computercontrolled motorized stepping stage (33).

\section{Proliferation assessment}

Cells were seeded in a 24-well culture plate (Greiner Bio One, 662160) and incubated under normal, serum starved or hypoxic conditions $\left(0.2 \% \mathrm{O}_{2}\right)$. CQ was used at a concentration of $5 \mu \mathrm{g} / \mathrm{ml}$ (Sigma Aldrich, C6628). Cellular density was determined using Cristal violet staining.

\section{Clonogenic survival assay}

Eight $h$ after counting and seeding, cells were exposed to serum-free medium or hypoxia $\left(\mathrm{O}_{2}<0.02 \%\right.$, Don Whitley, VA500) as described previously.(42) After exposure the medium was replaced with normal medium and cells were incubated under standard culture conditions until colonies formed (10 days). Colonies were visualized using $0.4 \%$ methylene blue in $70 \%$ ethanol staining. Only colonies consisting of $\geq 50$ cells were counted.

\section{Statistics}

Statistical analyses were performed using graphpad software. When comparing 2 conditions unpaired t-tests were used. For multiple comparisons, one way-ANOVA with Bonferroni corrections were used. p-values $<0.05$ were considered statistically significant.

\section{Results}

EGFRvIII-expressing cells are highly dependent on autophagy during starvation

EGFR is often overexpressed or mutated in diverse cancer types and especially in glioblastoma. One of the most commonly found mutations in EGFR is the constitutive active deletion variant EGFRVIII, which contributes to increased intrinsic radioresistance but also increases tolerance to microenvironmental factors that contribute to therapy resistance (e.g., hypoxia) $(24-26,43,44)$. The tumour microenvironment is characterized by the presence of regions that are low in oxygen (hypoxia) and nutrient supply. Previously we and others have shown that cells deprived of nutrients or oxygen rapidly activate 
autophagy $(31-33,45)$. Considering the high proliferation rate and nutritional demand of EGFRvIII-expressing cells, (46) we explored autophagic activity in EGFRvIII-expressing cells. Immunohistochemical analysis during serum-starved conditions revealed more and larger autophagosomes in the EGFRvIII-expressing cells compared to the control cells (Fig. 1A), suggesting changes in autophagic activity.

The gold standard in autophagy activity determination, is the measurement of autophagic flux. In short, during autophagy LC3B is partially degraded. Blocking lysosomal function prevents this degradation and the accumulation of this protein during these conditions reflects autophagic activity (flux). To achieve lysosomal inhibition, cells were exposed to nutrient deprivation in the absence and presence of the lysosomotropic drug, chloroquine $(\mathrm{CQ}, 5 \mu \mathrm{g} / \mathrm{ml})$. A faster and more pronounced induction of autophagy, as indicated by the accumulation of LC3B-II, was observed in the EGFRvIII-expressing U373 (Fig. 1B) and U-87 MG cells (Fig. 1C). Exposure to afatinib, an irreversible tyrosine kinase inhibitor, decreases autophagic flux in EGFRvIll-expressing cells in a dosedependent manner, indicating that the increased autophagic flux in EGFRvIII-expressing cells during starvation was EGFRvIII-activity dependent (Fig. S1A).

To determine if the higher autophagic flux contributed to growth advantages of EGFRvIll-expressing cells, cellular proliferation during normal and starvation conditions was determined. $\mathrm{CQ}$ addition had no significant effect on proliferation of either control or EGFRvIII-expressing cells during normal culture conditions (Fig. 1D), indicating that the used concentration was non-toxic at this dose. During starvation, EGFRvillexpressing cells proliferated faster compared to control cells (Fig. $1 E, p<0.05$ ). This growth advantage was abrogated by the addition of CQ to the culture medium. To assess whether autophagy inhibition alters survival of EGFRvIll-expressing cells, clonogenic survival after serum deprivation was determined. Serum deprivation did not significantly change clonogenic survival of control or EGFRvIII-expressing cells (Fig. 1F). However, CQ addition resulted in increased cell death of EGFRvIII-expressing cells $(p<0.05$ Control $+\mathrm{CQ}$ versus EGFRvIII $+\mathrm{CQ}$ at $\mathrm{t}=4$ ). Although $\mathrm{CQ}$ has been widely used as an inhibitor of autophagy, its effects are not solely restricted to the inhibition of autophagy. To confirm if the observed effects were dependent on autophagy, we engineered cells expressing a doxycycline-inducible shRNA directed against $L C 3 B$. In agreement with an autophagy dependence, targeting $\angle C 3 B$ sensitized cells to serum deprivation. In line with our pharmacological data, this effect was most pronounced in EGFRvIII-expressing cells (Fig. 1G, p<0.05).

Taken together, these data indicate that EGFRvIII-expressing cells activate autophagy to a higher extent and are highly dependent on autophagy for survival in response to serum deprivation. 


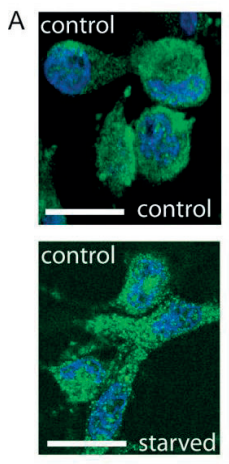

D

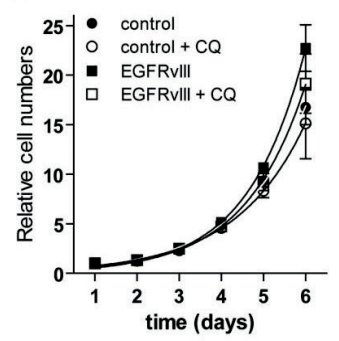

$\mathrm{F}$
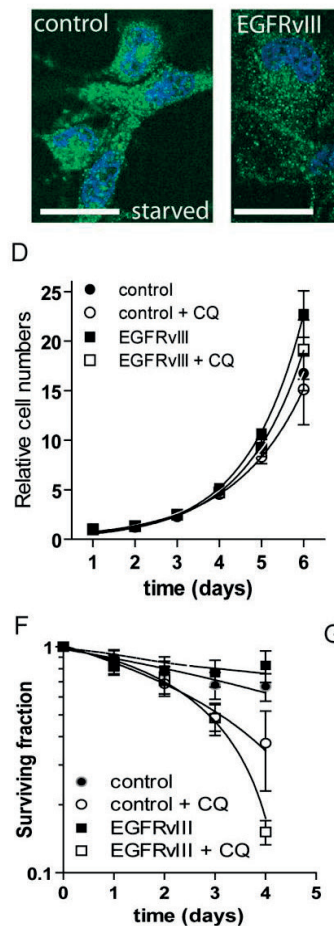
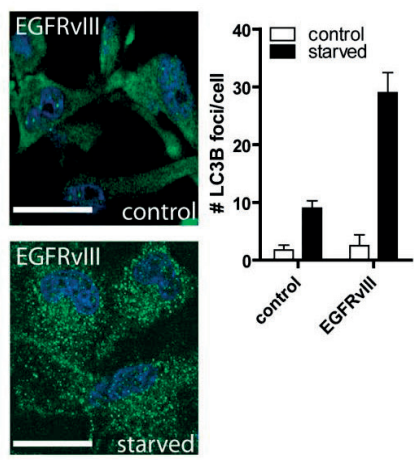

$\mathrm{E}$

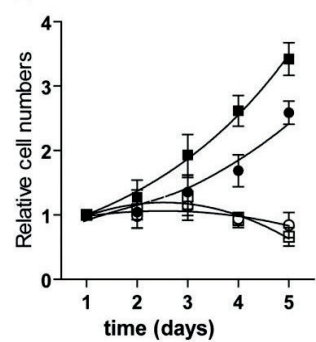

G

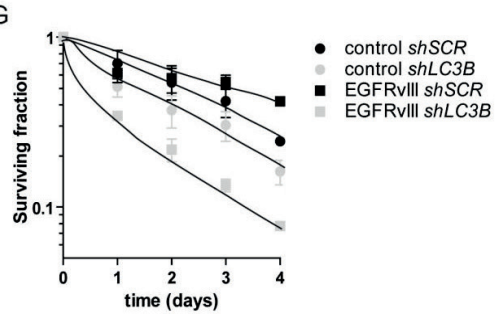

B

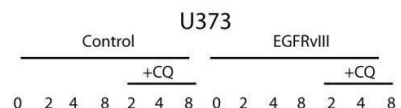

$\begin{array}{lllllllllllllll}0 & 2 & 4 & 8 & 2 & 4 & 8 & 0 & 2 & 4 & 8 & 2 & 4 & 8 & \text { starvation (h) }\end{array}$ EGFRvIII

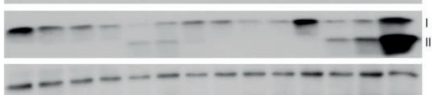
LC3B Actin

C

U87

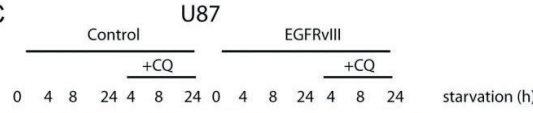

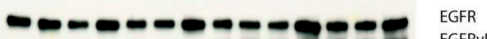
EGFRvIII LC3B-II ACTB

Figure 1. EGFRvIll-expressing cells display increased autophagy activation and dependence during serumstarved conditions.

(A) EGFRvIll-expressing cells display more and larger autophagosomes during starvation as assessed by immunofluorescence (nuclei [DAPI] in blue, LC3B in green). Scale bars: $10 \mu \mathrm{m}$. Immunoblot analysis of control and EGFRvIll-expressing cells reveal elevated autophagic flux during serum-starved conditions in EGFRvIIIexpressing U373 (B) and U87-MG (C) cells. Proliferation curves under normal (D) and serum-starved (E) conditions show that $C Q(5 \mu \mathrm{g} / \mathrm{ml})$ does not influence growth under normal conditions, but can abrogate the growth advantage of EGFRvIll-expressing cells during starvation. $n=3$, mean \pm SEM. Clonogenic survival assessment after serum starvation with or without addition of $C Q(\mathbf{F})$ or expression of a shRNA targeting $L C 3 B$ (G) indicate that the elevated survival advantage of EGFRvIll-expressing cells is autophagy dependent. $\mathrm{n}=3$, mean \pm SEM. SCR, scrambled control.

\section{EGFRvIll-expressing cells depend more on autophagy during hypoxia}

To assess if the enhanced autophagic response was restricted to serum deprivation or also applies to other types of metabolic stress present within the tumour microenvironment, we assessed the autophagic response and dependency on autophagy during hypoxic conditions. Similar to serum deprivation, EGFRvIII+ cells displayed an enhanced 
autophagic flux compared to the control cells as indicated by faster LC3B-II accumulation in the presence of $\mathrm{CQ}(5 \mu \mathrm{g} / \mathrm{ml})$ in U373 (Fig. 2A) and U-87 MG cells (Fig. 2B). This was further confirmed using tandem mCherry-EGFP-LC3B expression in U373 cells. Although no clear differences could be observed during normal culture conditions (not shown), after $16 \mathrm{~h}$ hypoxia exposure, EGFRvIII+ cells displayed mostly red foci whereas EGFRvIII- cells also displayed green/yellow foci (Fig. 2C). These data indicate faster processing of autophagosomes in EGFRvIII+ cells and support the flux analysis results seen by immunoblot.

To assess the dependency on autophagy for the survival of EGFRvIII-expressing cells, clonogenic survival after hypoxia exposure was determined in the presence of CQ (Fig. 2D) or after expressing a short hairpin that targets LC3B (Fig. 2E), or ATG7 (Fig. 2F) or after overexpression of dominant negative ULK1 ${ }^{\mathrm{K} 46 \mathrm{I}}$ (Fig. 2G). As reported previously, (12) EGFRvIll-expressing cells displayed a survival advantage in comparison to control cells, yet EGFRvIll-expressing cells were sensitized to hypoxia $\left(\mathrm{O}_{2}<0.02 \%\right)$ more than control cells in the presence of $\mathrm{CQ}$ or after targeting autophagy genetically (Fig. $2 \mathrm{C}$ and $D$, both $p<0.05$ at $t=3$ and/or -4 ; Fig. $2 F$ and $G, p<0.05$ ).

In addition to analysis of cells that ectopically express EGFRvIII, we explored the autophagy dependence of glioblastoma cells that endogenously express EGFRvIII. EGFRvIll-enriched populations were obtained after cell sorting (37). This way BS153 and DKMG isogenic cell populations have been obtained that lack EGFRvIII expression or are highly enriched for EGFRvIII expression (Fig. 2H). Also endogenous EGFRvIII+ cells displayed enhanced autophagic flux during hypoxia in BS153 (Fig. 2I) and DKMG (Fig. 2J) isogenic cell lines. This was further confirmed using mCherry-EGFP-LC3B expression in these cells. EGFRvIII+ cells displayed mostly mCherry-positive foci whereas EGFRvIII- also displayed yellow/green foci, suggesting a more rapid turnover of autophagosomes in EGFRvIII+ BS153 (Fig. 2K) and DKMG (Fig. 2L) cells. Due to their migratory character both BS153 and DKMG cells failed to form colonies. Therefore, the sensitivity of these cells to $\mathrm{CQ}(5 \mathrm{\mu g} / \mathrm{ml})$ addition during hypoxia was assessed by chrystal violet staining after exposure to hypoxia. During ambient air incubation, this concentration of CQ did not influence cell proliferation (Fig. S1B and C). As observed in ectopically expressing EGFRvIII+ cells, endogenously expressing EGFRvIII+ cells displayed a survival advantage during hypoxia in BS153 (Fig. 2M) and DKMG (Fig. 2N) cells. This survival advantage was fully abrogated in the presence of $\mathrm{CQ}$. These data indicate that cells are highly dependent on autophagy for survival during hypoxia after ectopic, but also endogenous, expression of EGFRvIII. 
A
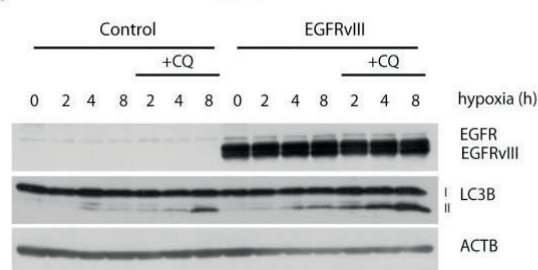

C
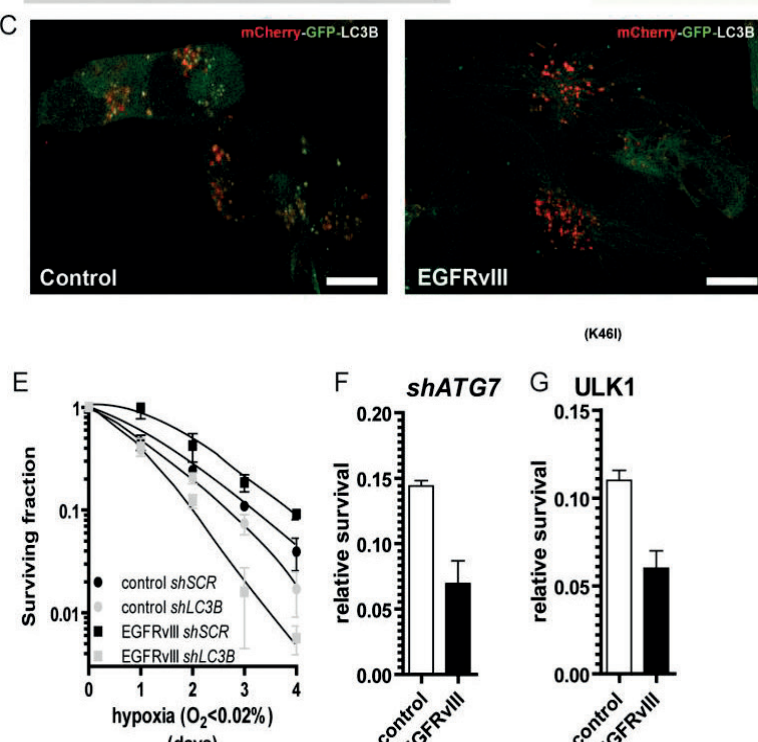

(days)
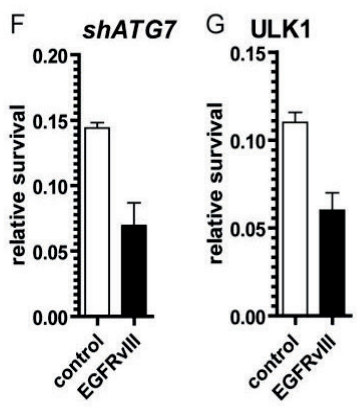

(K46I)

B

U87

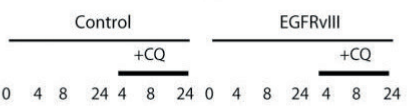

hypoxia (h)
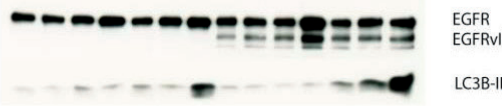

ACTB

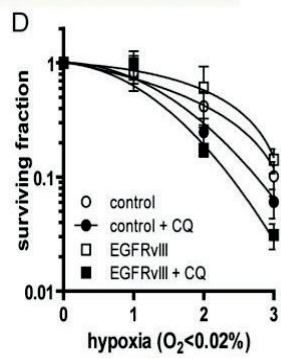

(days)

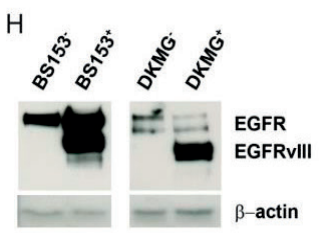

।

$J$ DKMG - DKMG ${ }^{+}$
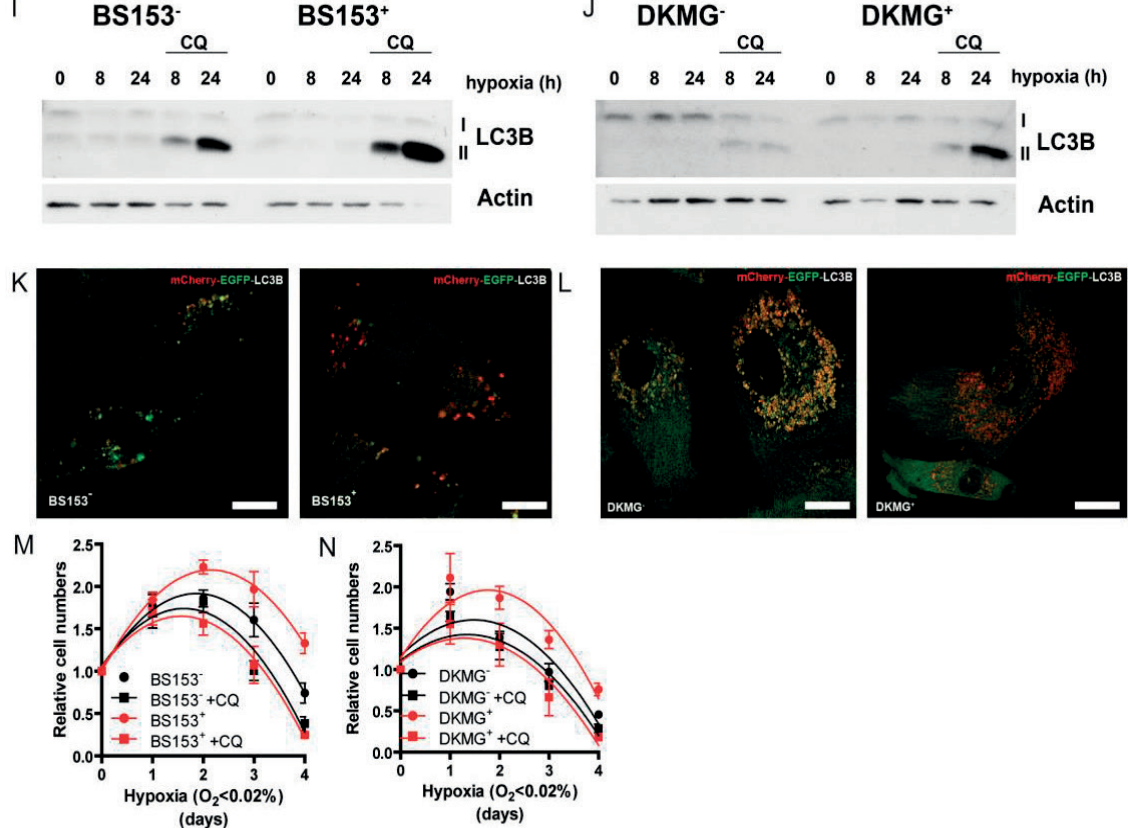
Figure 2. Inhibition of the elevated autophagic response in EGFRvIII-expressing cells abrogates their survival advantage during hypoxia. Immunoblot analysis indicates elevated autophagy activation in EGFRvIIIexpressing U373

(A) and U87 (B) cells during hypoxia. (C) Tandem GFP-mCherry-LC3B expression indicates elevated autophagic flux in EGFRvIII+ cells during hypoxia (24 $\mathrm{h} \mathrm{O}_{2}<0.02 \%$ ). Scale bars: $2 \mu \mathrm{m}$. Autophagy inhibition either through CQ addition $(5 \mu \mathrm{g} / \mathrm{ml})$ (D) or shRNA expression directed against $L C 3 B(\mathbf{E})$ abrogates the survival advantage of EGFRvIll-expressing cells as assessed by clonogenic survival assay. $n=3$, mean \pm SEM (F) Targeting Atg7 through expression of a shRNA (G) or ULK1 through expression of dominant negative ULK1 ${ }^{\mathrm{K} 461}$ results in loss of survival advantage of EGFRvIII ${ }^{+}$cells as determined by clonogenic survival assay after $48 \mathrm{~h}$ hypoxia $\left(\mathrm{O}_{2}<0.02 \%\right)$. $\mathrm{n}=3$, mean \pm SEM $(\mathbf{H})$ EGFR and EGFRvIII expression of BS153 and DKMG cell populations. Autophagic flux determination in (I) BS153 and (J) DKMG cells during hypoxia. Tandem GFP-mCherry-LC3B expression indicates elevated autophagic flux in EGFRvIII+ (K) BS153 and (L) DKMG cells during hypoxia $\left(24 \mathrm{~h} \mathrm{O}_{2}<0.02 \%\right.$ ). Scale bars: $2 \mu \mathrm{m}$. Autophagy inhibition through CQ addition $(5 \mu \mathrm{g} / \mathrm{ml})$ abrogates the survival advantage of EGFRvIII ${ }^{+}$ (M) BS153 and (N) DKMG cells as assessed by crystal violet staining after hypoxia exposure. $n=3$, mean \pm SEM.

\section{EGFRvIII-expressing xenografts depend on autophagy for accelerated regrowth after therapy}

To investigate the higher dependency of EGFRvIll-expressing cells on autophagy during metabolic stresses in vivo, control and EGFRvill-expressing tumour xenografts were grown in nude mice (Fig. $3 \mathrm{~A}$ ) and treated with CQ (60 mg/kg daily). In line with previous results, (12) EGFRvIll tumours displayed accelerated growth compared to control tumours (Fig. 3A). Interestingly, 7-day CQ treatment resulted in decreased growth (Fig. 3B; $20 \%$ increase in doubling time for control tumours and 30\% increase for EGFRvIII expressing tumours, both $\mathrm{p}<0.05)$. EGFRvIll expression was validated by immunohistochemistry (Fig. 3C).

$\mathrm{CQ}$ treatment changes the tumour microenvironment by decreasing the hypoxic fraction through reduced hypoxia tolerance (33) and increased perfusion (47). Our in vitro data suggest that EGFRvIII-expressing cells were more dependent on autophagy for survival during hypoxia (Fig. 2). In line with the observed increased intrinsic tolerance of EGFRvIll-expressing cells to hypoxia (Fig. 2 and ref. (12)), EGFRvIll-expressing tumours displayed a higher hypoxic fraction compared to control tumours as determined by pimonidazole immunohistochemistry (Fig. 3D). In both control tumours and EGFRVIII-expressing tumours CQ treatment resulted in a decrease in tumour hypoxia (Fig. 3D; $p<0.05$ in control, $p<0.01$ for EGFRvlll tumours), although this effect was most pronounced in EGFRvIll-expressing tumours.

As expected, cellular proliferation, as determined by BrdU incorporation, was higher in EGFRvIll-expressing tumours compared to control tumours (Fig. S2A), but was unaffected by $C Q$ treatment, indicating that hypoxic cells contribute very little to proliferation in the tumour. In control tumours, $C Q$ treatment increased vessel density $(p<0.05)$ and may have contributed to the reduction in tumour hypoxia (Fig. S2B). In contrast, EGFRvIll tumours showed a high vessel density when untreated, but there CQ treatment reduced, although to a limited extent $(18 \%)$, the vessel density $(p<0.05)$. These results suggest that the effect of CQ on vessel function and abundance is phenotype depend- 
ent. No differences in perfused vessels in either of the tumours were observed (Fig. S2C). In contrast, the necrotic fraction increased significantly after CQ treatment in EGFRvIll-expressing tumours (2.3 fold, $p=0.01$ ), but not in control tumours (Fig. 3E), suggesting that CQ treatment leads to cell death of EGFRvIII-expressing cells in vivo.

\section{A Tumor growth}

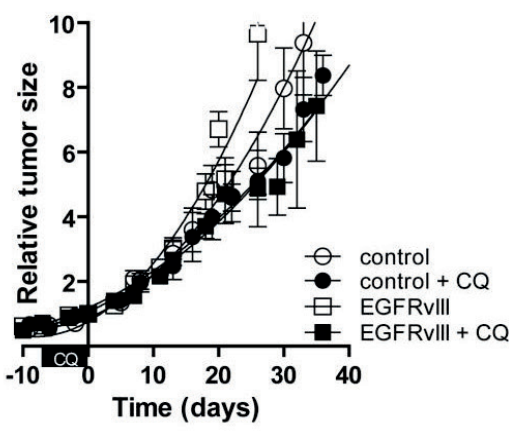

B doubling time
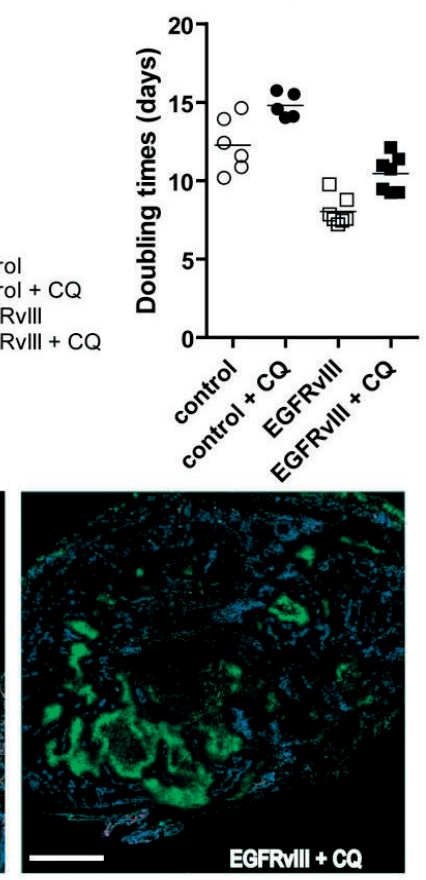

EGFRUII + CQ

$\mathrm{E}$
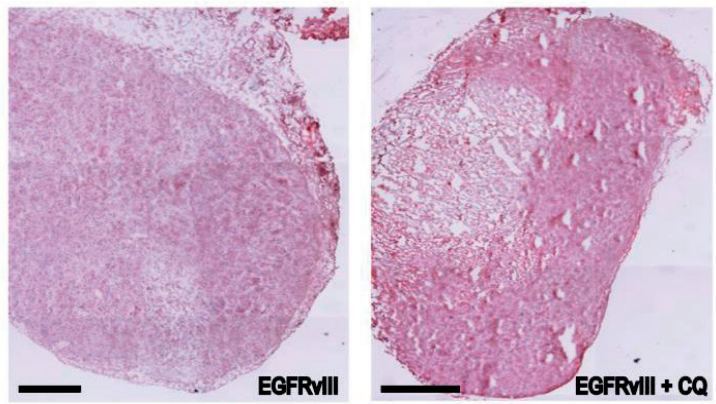

D

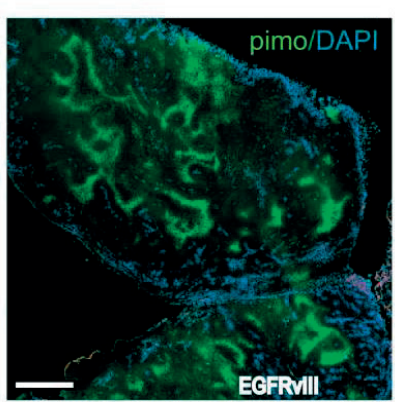

C Control
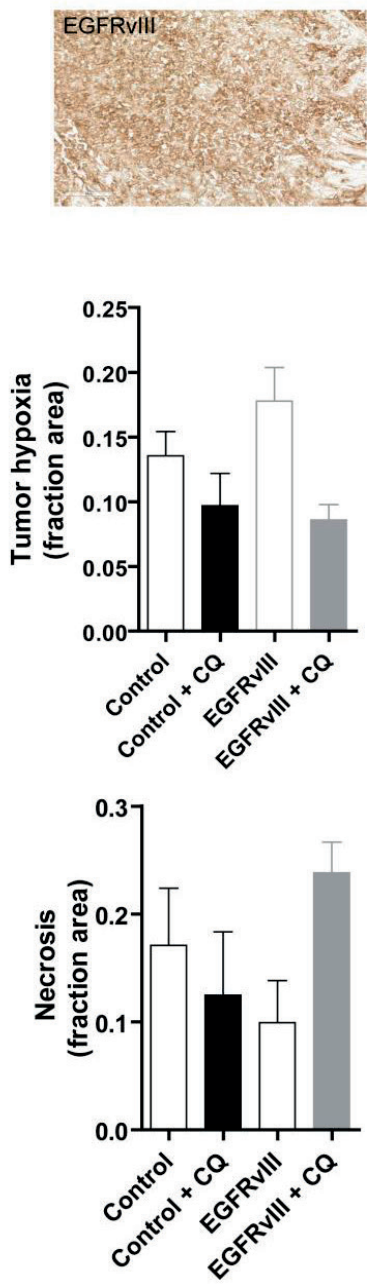

Figure 3. $C Q$ treatment reduces tumour growth and tumour hypoxia and increases tumour necrosis in EGFRvIll-expressing tumours. 
(A) Growth curves of control U373 xenografts (open circles, $n=6$ ) or treated from day -7 to 0 with $\mathrm{CQ}$ (60 $\mathrm{mg} / \mathrm{kg}$ ) (filled circles, $\mathrm{n}=5$ ) and EGFRvlll-expressing U373 xenografts (open squares, $\mathrm{n}=7$ ) treated with CQ (filled squares, $n=7$ ). (B) Doubling times of the corresponding tumours. (C) Immunohistochemical staining for EGFRvIII on control (upper panel) and EGFRvIII-expressing xenografts (lower panel). Scale bars: 2 mm. (D) (Left panel) Micrographs of pimonidazole-DAPI staining prior to and after 7 days CQ treatment on EGFRvIIIexpressing tumours. (Right panel) Quantification of the tumour hypoxic fraction (control $n=6$, control $+\mathrm{CQ}$ $n=6$, EGFRvIII n=5, EGFRvIII +CQ n=5). (E) (Left panel) Micrographs of hematoxylin and eosin staining prior to and after 7 days CQ treatment on EGFRvIII-expressing tumours. (Right panel) Quantification of tumour necrosis (control $n=6$, control + CQ n=6, EGFRvIII n=5, EGFRvIII + CQ n=5).

Hypoxic cells contribute to radiotherapy resistance; we therefore determined whether the decrease in hypoxia after CQ administration could sensitize EGFRvIll-expressing tumours to irradiation. Control and EGFRvIII tumour bearing mice were treated with CQ for 7 days prior to irradiation and tumour regrowth was monitored. As expected, growth of control tumours was significantly delayed after a single, tumour specific, dose of $10 \mathrm{~Gy}$ and could be further delayed by CQ pre-treatment (Fig. 4A). EGFRvIIIexpressing tumours respond faster to irradiation, often an indication of a different type of cell death(48) but also regrow faster. Yet the effect of CQ pre-treatment on EGFRvIIIexpressing tumours postponed regrowth even further (Fig. 4A). To illustrate the differences in radiation sensitivity of the individual tumours, the data are replotted in Fig. 4B as Kaplan-Meier plots. Tumour irradiation extended the median survival of mice with control tumours from 20 to 31 days, and $C Q$ pre-treatment increased median survival further to 40 days. The effect of CQ addition in EGFRvIII-expressing tumours was much larger as irradiation extended survival from 15 to 25 days, but in combination with CQ pre-treatment this survival was extended to 48 days (Fig. 4B). This effect was even more pronounced after a larger irradiation dose (15 Gy), showing an additional increase from 61 to 108 days after CQ treatment prior to irradiation (Fig. S3).

Expression of EGFRvIII on GBM cells is rapidly lost when cultured in vitro, suggesting a survival and/or selection advantage for EGFRvIll-expressing cells in the tumour microenvironment. For this reason most studies on EGFRvIII are performed in established GBM cell lines in which EGFRvIII is ectopically expressed. These cells and xenografts might behave different than the original tumours as these cells are clearly not dependent on EGFRvIII signalling for survival or growth, and expression levels of EGFRvIII may be much higher than endogenous levels. We have therefore generated primary tumour xenografts directly derived from a cancer patient (patient derived xenograft; PDX), with an EGFRvIII-positive GBM. These primary tumour xenografts are thought to better resemble and reflect the original tumour and its response to therapy. After growth as a xenograft, EGFRvIII expression was retained over generations, as determined by EGFRvIll-specific immunohistochemistry (Fig. 4C) and a substantial hypoxic fraction was observed (Fig. 4D, mean \pm Stdev $0.15 \pm 0.04, n=6$ ). Similar to the ectopically EGFRvIIIexpressing tumours, $C Q$ administration reduced tumour growth (18 to 28 days doubling time $p<0.05$ ) in endogenously expressing EGFRvIII-positive PDX (Fig. 4E), which resulted in an increase in median survival from 32 to 40 days (Fig. 4F). Tumour irradiation in- 
creased median survival to 66 days and could be increased by CQ administration to 102 days post treatment. Together, these data indicate that autophagy is a therapeutic vulnerability of EGFRvIII-expressing GBM that can be exploited by CQ administration.
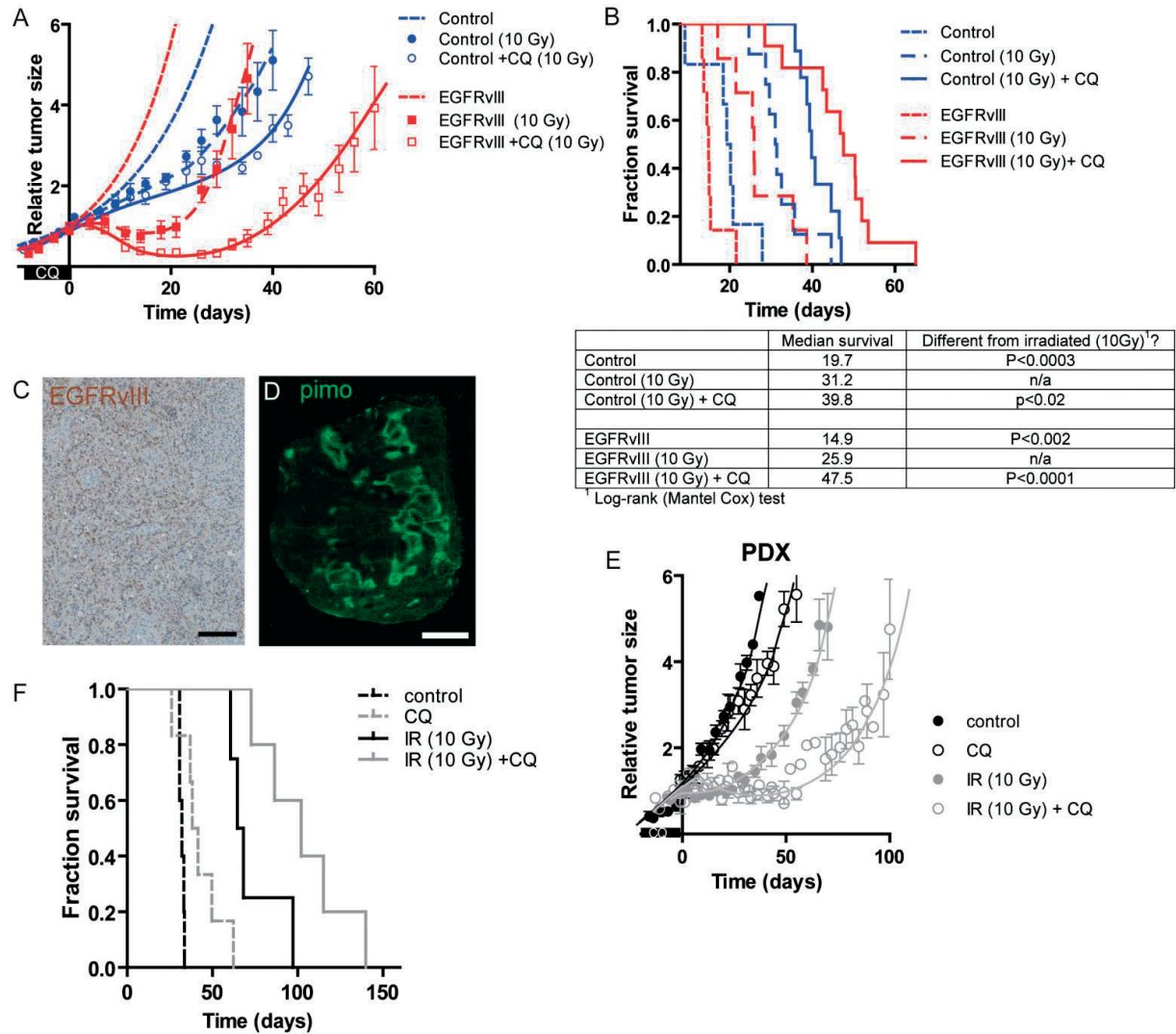

E

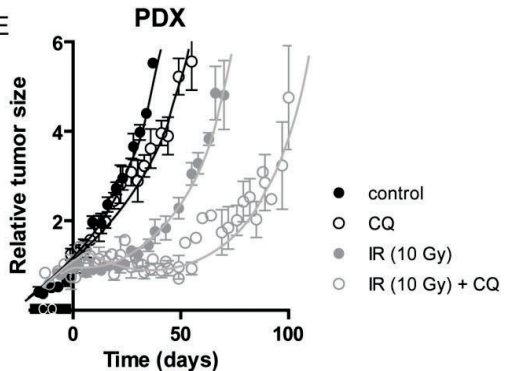

\begin{tabular}{|l|c|l|}
\hline & Median survival & Statistics $^{1}$ \\
\hline Control & 32.1 & \\
\hline $\mathrm{CQ}$ & 39.9 & $\mathrm{p}<0.02$ vs Control \\
\hline $\mathrm{IR}(10 \mathrm{~Gy})$ & 66.4 & $\mathrm{p}<0.005$ vs Control \\
\hline IR $(10 \mathrm{~Gy})+\mathrm{CQ}$ & 102.0 & $\mathrm{p}<0.04$ vs IR (10Gy) \\
\hline \multicolumn{2}{|l}{${ }^{1}$ Log-rank (Mantel Cox) test }
\end{tabular}

Figure 4. $C Q$ treatment sensitizes EGFRvIII-expressing tumours to irradiation.

(A) Growth curves of U373 xenografts: untreated (dashed lines; control [blue, n=6], EGFRvIII[(red, $n=7]$ ), treated with a single, tumour-specific dose of $10 \mathrm{~Gy}$ at $\mathrm{t}=0$ (interrupted line; control [blue, $\mathrm{n}=8$ ], EGFRvIII [red, $\mathrm{n}=7 \mathrm{]})$ or irradiated after 7 days $\mathrm{CQ}(60 \mathrm{mg} / \mathrm{kg})$ pre-treatment from $\mathrm{t}=-7$ to $\mathrm{t}=0$ and irradiated with a single, tumour-specific dose of $10 \mathrm{~Gy}$ at $\mathrm{t}=0$ (solid lines; control [blue, $\mathrm{n=9}$ ], EGFRvIII [red $\mathrm{n=11}$ ]). (B) Kaplan-Meier representations of the time to reach $4 x$ the treated volume (from $t=0)$. Immunohistochemical staining for $(\mathbf{C})$ EGFRvIII (scale bar: $50 \mu \mathrm{m}$ ) and (D) pimonidazole of PDX (scale bar: $2 \mathrm{~mm}$ ), indicating EGFRvIII positivity and a substantial hypoxic fraction. (E) Growth curves of the PDX untreated (black closed circles, $n=5$ ), treated with $\mathrm{CQ}(60 \mathrm{mg} / \mathrm{kg})$ for 7 days from $\mathrm{t}=-7$ to $\mathrm{t}=0$ (open circles, $\mathrm{n}=5$ ), treated with a single, tumour-specific dose, of $10 \mathrm{~Gy}$ at $\mathrm{t}=0$ (gray closed circles, $\mathrm{n}=4)$ and treated with $\mathrm{CQ}(60 \mathrm{mg} / \mathrm{kg})$ for 7 days from $\mathrm{t}=-7$ to $\mathrm{t}=0$ followed by a single, tumour-specific dose, of $10 \mathrm{~Gy}$ at $\mathrm{t}=0$ (gray open circles, $\mathrm{n}=5$ ). ( $\mathbf{F}$ ) Kaplan-Meier representations of the time to reach $4 x$ the treated volume (from $t=0$ ). 
EGFRvIII-expressing cells are dependent on autophagy due to a high metabolic demand

As EGFRvIII-expressing cells appear to be more dependent on autophagy for their survival during metabolic stress, we hypothesized that autophagy supports the high metabolic demand of EGFRvIll-expressing cells.(46) We therefore assessed glucose abundance in the medium of control and EGFRvIll-expressing cells. Although glucose consumption was higher under normal culture conditions in EGFRvIII-expressing cells, the addition of $\mathrm{CQ}(5 \mu \mathrm{g} / \mathrm{ml})$ did not change glucose consumption under ambient oxygen conditions (Fig. 5A, $p<0.05$ at $t=24$ ). As expected, glucose consumption increased during hypoxia (Fig. 5A). Interestingly, CQ addition did not change glucose consumption of control cells, but increased glucose consumption by EGFRvIII-expressing cells (Fig. 5A). Comparable to $C Q$, inhibition of autophagy through targeting $\angle C 3 B$, increased glucose consumption in EGFRvIII-expressing cells (Fig. 5B) during hypoxia, but also increased glucose consumption in control cells. This illustrates that besides autophagy, CQ and LC3B may also be involved or influence other processes.

The increased glucose metabolism after inhibition of autophagy during hypoxia in EGFRvIII cells, suggests that autophagy is used as an alternative mechanism required in support of the high metabolic demand and energy production. We therefore questioned whether the elevated autophagic flux in EGFRvIII cells during hypoxia is required to meet this demand. We therefore assessed autophagic flux after provision of additive external resources (glucose and pyruvate) for metabolic processing. In control cells, no changes in flux were observed after addition of glucose and/or pyruvate (Fig. 5C). In contrast, in EGFRvIII-expressing cells, addition of pyruvate, but not glucose, rescued the elevated autophagic flux. We hypothesized that failure to meet the high metabolic demand, for example through autophagy inhibition, results in cell death of EGFRvIIIexpressing cells. To test this, we exposed control and EGFRvIII-expressing cells to hypoxia for $48 \mathrm{~h}$ in the presence or absence of CQ (Fig. 5D) or short hairpins targeting $L C 3 B$ (Fig. 5E), supplemented the culture medium with additional pyruvate and assessed clonogenic survival. Interestingly, the addition of pyruvate rescued cell death in autophagy-deficient EGFRvIII-expressing cells ( $p<0.01$ in Fig. 5D and E), but not in control cells. This illustrates the therapeutic potential of targeting the high metabolic demand of EGFRvIII-expressing cells and tumours through inhibition of autophagy. 
A
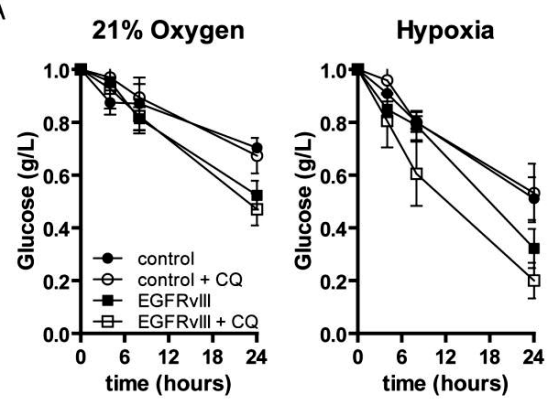

B Hypoxia

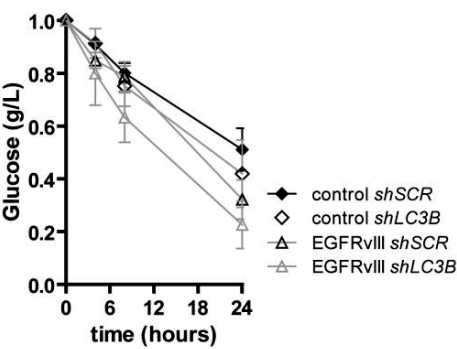

C control EGFRvill

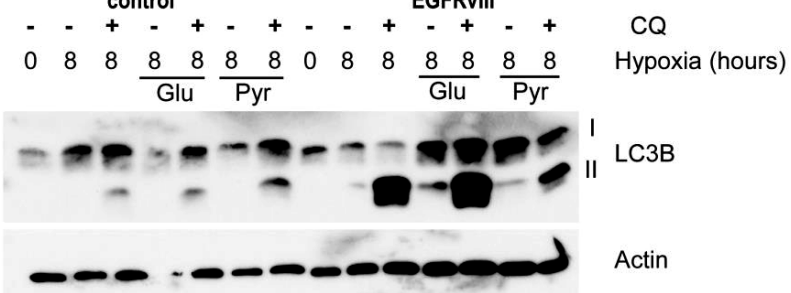

D

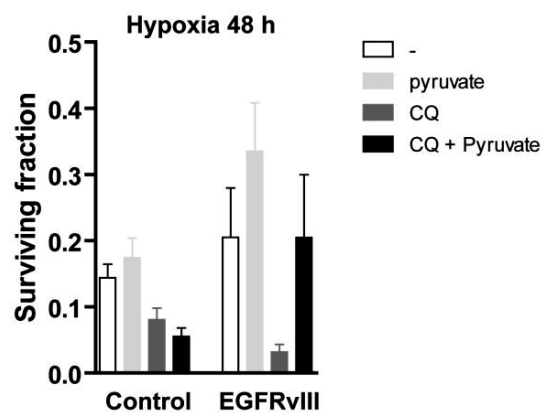

E

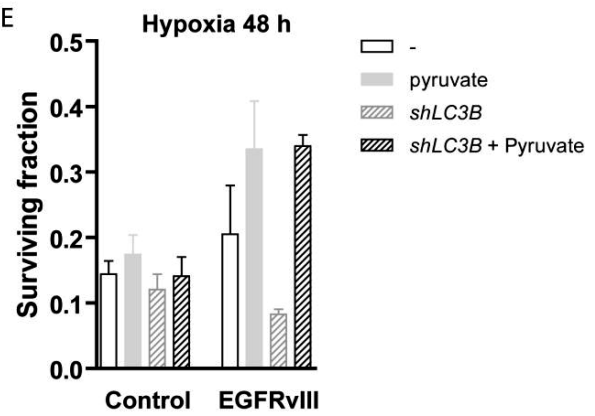

Figure 5. EGFRvIII-expressing cells require autophagy to meet high metabolic demand.

(A) Glucose utilization by control and EGFRvIll-expressing U373 cells under ambient (left) or hypoxic $\left(\mathrm{O}_{2}<0.02 \%\right)$ (right) conditions with or without addition of $\mathrm{CQ}(5 \mathrm{\mu g} / \mathrm{ml})$. $\mathrm{n}=3$, mean $\pm \mathrm{SEM}$. (B) Glucose consumption after expression of a control shRNA or a shRNA targeting $\angle C 3 B$. $n=3$, mean \pm SEM (C) Autophagic flux determination in control and EGFRvIll-expressing cells after 8-h hypoxia exposure with additional glucose or methyl pyruvate supplementation $(5 \mathrm{mM})$. (D) Clonogenic survival after $48 \mathrm{~h}$ hypoxia $\left(\mathrm{O}_{2}<0.02 \%\right)$ exposure in the presence of $\mathrm{CQ}$, methyl pyruvate or $\mathrm{CQ}$ and pyruvate. $\left(\mathrm{O}_{2}<0.02 \%\right) \mathrm{n}=3$, mean $\pm \mathrm{SEM}$. (E) Clonogenic survival after 48 -h hypoxia $\left(\mathrm{O}_{2}<0.02 \%\right)$ exposure in control and LC3B-depleted cells with pyruvate supplemented $\mathrm{n}=3$, mean \pm SEM.

\section{Patients with EGFRvIII-positive GBM benefit from CQ treatment}

Several reports have been published on the prognostic role of EGFRvIII in GBM patients. Although most reports indicate worse survival for EGFRvIII+ GBM patients, others have indicated a different outcome. This difference may depend on the method of EGFRvIII detection either through PCR or immunohistochemistry or on the heterogeneity of the 
patient populations and therapeutic interventions included. We therefore selected 57 patients with a primary GBM, recently treated in our institution. In this cohort, 30 (53\%) of the tumours were identified to express EGFRvIII through immunohistochemical analysis (Fig. 6A). All patients were treated with TMZ (75 mg per square meter of bodysurface area per day) and radiotherapy (total dose $60 \mathrm{~Gy}, 2$ fractions per day). In this very homogeneously treated patient cohort, immunohistochemical detection of EGFRvIII expression confirmed earlier reports $(14,49)$ which indicate significantly worse prognosis for EGFRvIII-expressing GBM patients (Fig. 6B, p<0.05). Patient characteristics are listed in Table 1.

\section{A}
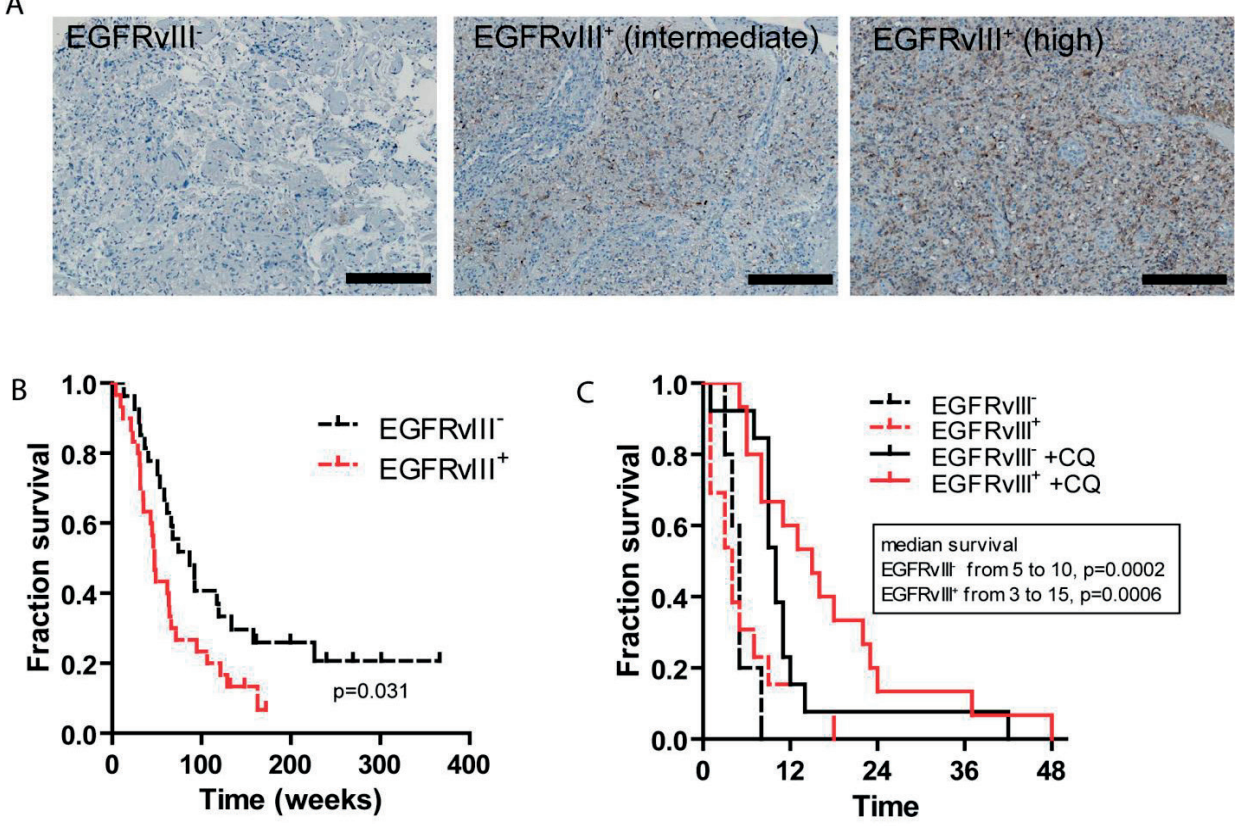

Figure 6. Patients with EGFRvIII-expressing GBM benefit most from concurrent CQ treatment.

(A) Micrographs of immunohistochemically stained biopsy slides of a negative (left) and EGFRvIII-expressing tumours (middle and right). Scale bar: $100 \mu \mathrm{m}$. (B) Kaplan-Meier survival plots of $61 \mathrm{GBM}$ patients after TMZradiotherapy treatment, separated based on EGFRvIII expression. (C) Survival plot of 43 GBM patients categorized for EGFRvIII status and CQ treatment. Patients with an EGFRvIII-positive tumour benefit substantially from CQ treatment.

To assess the clinical relevance of our findings in the treatment of EGFRvIll-expressing GBM, we retrospectively analyzed 43 GBM patients that were included in a randomized, placebo-controlled phase II trial (36). Of the analyzed patients, 28 patients received CQ in combination with carmustine and radiotherapy. EGFRvIII expression was assessed immunohistochemically and observed in 22 of 43 (51\%) patients. Patient characteristics are listed in Table 2. Although the number of analyzed patients is low, these results are 
encouraging as $C Q$ treatment improved median survival from 5 to 10 months in patients with EGFRvIII-negative GBM (Fig. 6C, p=0.0002). Strikingly median survival of patients with EGFRvIII-positive GBM increased from 3 to 15 months ( $p=0.0006)$, confirming our pre-clinical findings and the therapeutic potential of CQ in the treatment of EGFRvIIIexpressing tumours.

Table 1. Characteristics of GBM patients treated with $T M Z$ and radiotherapy (60 Gy)

\begin{tabular}{llc}
\hline EGFRvIII status & EGFRvIII- & EGFRvIII+ $^{+}$ \\
\hline & $27(47 \%)$ & $30(53 \%)$ \\
Male & $15 / 27$ & $19 / 30$ \\
Female & $12 / 27$ & $11 / 30$ \\
Karnofsky performance (pre-surgery) & & \\
$60-70$ & $0(0 \%)$ & $2(7 \%)$ \\
$80-90$ & $8(30 \%)$ & $19(63 \%)$ \\
100 & $19(70 \%)$ & $9(30 \%)$ \\
TMZ (75 mg/m²/day) & $27 / 27(100 \%)$ & $30 / 30(100 \%)$ \\
Radiotherapy (60 Gy) & $27 / 27(100 \%)$ & $30 / 30(100 \%)$ \\
\hline
\end{tabular}

Table 2. Characteristics of GBM patients

\begin{tabular}{|c|c|c|c|c|}
\hline \multirow[b]{2}{*}{ EGFRvIll status } & \multicolumn{2}{|c|}{ no CQ treatment } & \multicolumn{2}{|l|}{$\mathrm{CQ}$ treatment } \\
\hline & vIII- & $\mathrm{vIII} \mathrm{I}^{+}$ & $\mathrm{vIII}^{-}$ & vIII+ \\
\hline Male & $5 / 8$ & $3 / 7$ & $10 / 13(77 \%)$ & $6 / 15(40 \%)$ \\
\hline Female & $3 / 8$ & $4 / 7$ & $3 / 13(23 \%)$ & $9 / 15(60 \%$ \\
\hline \multicolumn{5}{|c|}{ Karnofsky performance (pre-surgery) } \\
\hline$<70$ & $0(0 \%)$ & $1(14 \%)$ & $0(0 \%)$ & $1(7 \%)$ \\
\hline $70-80$ & $7(87 \%)$ & $6(86 \%)$ & $7(54 \%)$ & $7(47 \%)$ \\
\hline $90-100$ & $1(13 \%)$ & $0(0 \%)$ & $6(46 \%)$ & $7(47 \%)$ \\
\hline \multicolumn{5}{|c|}{ Karnofsky performance (post-surgery) } \\
\hline$<70$ & $1(13 \%)$ & $2(29 \%)$ & $0(0 \%)$ & $0(0 \%)$ \\
\hline $70-80$ & $1(13 \%)$ & $1(14 \%)$ & $1(8 \%)$ & $3(20 \%)$ \\
\hline $90-100$ & $6(74 \%)$ & $4(57 \%)$ & $12(92 \%)$ & $12(80 \%)$ \\
\hline 2nd surgery & $4 / 8(50 \%)$ & $2 / 7(29 \%)$ & $2 / 13(15 \%)$ & $2 / 15(13 \%)$ \\
\hline Radiotherapy & $3 / 8(38 \%)$ & $2 / 7(29 \%)$ & $8 / 13(62 \%)$ & $6 / 15(40 \%)$ \\
\hline
\end{tabular}

\section{Discussion}

Our data confirmed that the expression of EGFRvIII indicates a poor prognosis for GBM patients due to increased therapy resistance, increased proliferation and accelerated tumour growth. Furthermore, EGFRvIII-expressing cells are more resistant to metabolic stresses and result in an increased hypoxic fraction of the tumour. As tumour hypoxia is a major limiting factor in successful cancer treatment,(27) targeting hypoxia in EGFRvIII- 
expressing tumours may therefore be essential in acquiring improved tumour control and ultimately tumour cure. Here we reveal that expression of EGFRvIII leads to increased autophagic flux during metabolic stresses such as hypoxia and starvation. In line with previous reports, EGFRvIII-expressing cells proliferate faster and display increased survival during these stresses (10-13). We show that these advantages can be abrogated through inhibition of autophagy either through genetic or pharmacological targeting. In vivo, EGFRvIII tumours display increased tumour growth and faster tumour regrowth after radiotherapy, effects that are likely to contribute to the poor outcome of EGFRvIII+ GBM patients. Importantly, CQ treatment inhibits the accelerated growth and regrowth of EGFRvIII tumours. As observed previously, (33) CQ treatment results in reduced tumour hypoxia in tumour xenografts, yet we observed that the largest reduction occurs in EGFRvIll-expressing tumours. Strikingly, CQ treatment results in a drastic increase in necrosis of EGFRvIII tumours, but not in control tumours. In addition to lowering the intrinsic tolerance to hypoxia, CQ may induce vessel normalization and increase perfusion, resulting in decreased tumour hypoxia (47). In our experiments, vessel density increased in control tumours but not in EGFRvIII-expressing tumours (Fig. S2). Our data indicates that EGFRvIII expression changes the tumour cell dependence on autophagy to tolerate low oxygen levels. In the absence of autophagy, these cells will die leading to increased tumour necrosis. These results are in line with the enhanced cell death after autophagy inhibition in EGFRvIll-expressing cells, in vitro (Fig. 2C and D).

During autophagy, recycled products are transported across the autolysosomal membrane into the cytoplasm. These metabolites can be reused in protein and ATP production (34). In our studies we observed that EGFRvIII+ tumours cells displayed increased activation and dependence on autophagy during hypoxia, effects that can be rescued by adding pyruvate to the culture medium. As EGFRvIII+ cells consume more glucose (Fig. 5), autophagy may be essential in supporting metabolic processes in EG$\mathrm{FRvIII}^{+}$cells. EGFRvIII may induce the increased glucose uptake and consumption through enhanced RRAS signalling. Similar to our observations, oncogenic RRAS promotes glucose uptake and utilization in glycolysis (reviewed in ref. (50)). Interestingly, the effect of autophagy inhibition in EGFRvIII+ cells on survival and glucose consumption during hypoxia could not be rescued by glucose addition, but was rescued after pyruvate supplementation. This suggests that a) the glycolytic pathway is used to its fullest extent, and b) the high metabolic activity is not used to support ATP production, as pyruvate cannot further be degraded in the citric acid cycle due to the absence of oxygen. In addition to being converted to ATP, pyruvate is able to prevent toxic effects of oxygen radicals and peroxides and indirectly prevents the depletion of glutathione during hypoxia, (51) and may therefore decrease the toxic effects after reoxygenation.

From a clinical perspective, the most important finding of this research is the relatively large effect of $\mathrm{CQ}$ on tumours that express EGFRvIII compared to the nonEGFRvIII-expressing tumours. In cell line-derived xenografts, we observed transition of the hypoxic into necrotic areas and a high degree of sensitization to radiotherapy. These 
results were further confirmed in patient-derived xenografts. Furthermore, retrospective analysis of EGFRvIII expression in GBM patients treated with carmustine, radiotherapy and surgery revealed a larger improvement in survival for patients with EGFRvIIIpositive GBM after concurrent CQ treatment compared to patients with EGFRvIIInegative tumours. Although these initial clinical results are promising, larger cohorts of patients are required to fully understand the therapeutic potential of $C Q$ administration in the treatment of GBM. Recently a phase I/II trial in newly diagnosed GBM has been reported where standard of care (temozolomide and radiotherapy) was combined with concurrent hydroxychloroquine (a derivative of CQ) (52). Due to unfavourable toxicity profiles and dose-limiting toxicity before consistent inhibition of autophagy was observed, no changes, compared to historical data (EORTC trial), in overall survival were observed. Although hydroxychloroquine and chloroquine belong to the same class of 4aminoquinolines, their effective doses and toxicity profiles differ. Clinical trials to evaluate toxicity and effectiveness (randomized phase II trial) of $\mathrm{CQ}$ in combination with temozolimide and radiotherapy are ongoing in our institute (clinicaltrials.gov identifier NCT02378532 and NCT02432417). Based on the findings described in this manuscript, in the phase II trial patients will be stratified based on expression of EGFRvIII.

Interestingly, other mutation types in the RAS-RAF-MAPK/ERK pathway have also been associated with increased dependence on autophagy for survival. For example, HRAS $^{G 12 V}$ or KRAS ${ }^{G 12 V}$ transformed cells upregulate their basal autophagy and depend on elevated autophagy for survival during starvation (53). Also the BRAF ${ }^{\mathrm{V} 600 \mathrm{E}}$ mutation, which occurs in over $50 \%$ of childhood central nervous system tumours, results in elevated autophagy and increased sensitivity to pharmacological or genetic autophagy inhibition in terms of cell viability.(54) Remarkable clinical responses have been observed.

These tumours are often also effectively treated using kinase inhibitors, such as vemurafenib. Unfortunately, development of resistance is often observed. Levy et al. reported that autophagy contributes to vemurafenib resistance. Hence, targeting autophagy can overcome this resistance, and patients with resistant tumours display favourable clinical responses when chloroquine is combined with vemurafenib (55). Combined with our data these results suggest that constitutive signalling through the RASRAF-MAPK/ERK pathway creates autophagy dependence in tumour cells. This important information could be used for individualized treatment selection for patients with brain tumours.

In conclusion, our results demonstrate that elevated autophagy activity contributes to the enhanced tolerance to metabolic stresses of EGFRvIII-expressing cells. Targeting this survival mechanism abrogates this advantage and results in enhanced tumour cell killing. Ultimately this downregulation of autophagy activity leads to reduced tumour growth, reduced tumour hypoxia, increased tumour necrosis and sensitization of tumours to radiotherapy. In line with these pre-clinical findings, we observed that patients with EGFRvIII-expressing GBM benefit most from concurrent CQ treatment. Concurrent 
$\mathrm{CQ}$ treatment in combination with conventional therapy seems therefore a promising strategy to improve the outcome for EGFRvIII+ GBM patients.

\section{Acknowledgements}

This work was financially supported by the Dutch Cancer Society (KWF Grants UM 20125506 and 2015-7735 to K.R.), worldwide cancer research award 16-0265 (to K.R.) and STOPhersentumouren.nl/rare diseases fund (to K.R.). 


\section{References}

1. Lemmon MA, Bu Z, Ladbury JE, Zhou M, Pinchasi D, Lax I, et al. Two EGF molecules contribute additively to stabilization of the EGFR dimer. The EMBO journal 1997;16:281-94

2. Linggi B, Carpenter G. ErbB receptors: new insights on mechanisms and biology. Trends in Cell Biology 2006;16:649-56

3. Nicholson RI, Gee JM, Harper ME. EGFR and cancer prognosis. European journal of cancer 2001;37 Suppl 4:S9-15

4. Dittmann K, Mayer C, Fehrenbacher B, Schaller M, Kehlbach R, Rodemann HP. Nuclear EGFR shuttling induced by ionizing radiation is regulated by phosphorylation at residue Thr654. FEBS letters 2010;584:3878-84

5. Schmidt-Ullrich RK, Mikkelsen RB, Dent P, Todd DG, Valerie K, Kavanagh BD, et al. Radiation-induced proliferation of the human A431 squamous carcinoma cells is dependent on EGFR tyrosine phosphorylation. Oncogene 1997;15:1191-7

6. Ge H, Gong X, Tang CK. Evidence of high incidence of EGFRvIII expression and coexpression with EGFR in human invasive breast cancer by laser capture microdissection and immunohistochemical analysis. International journal of cancer Journal international du cancer 2002;98:357-61

7. Jutten B, Dubois L, Li Y, Aerts H, Wouters BG, Lambin P, et al. Binding of cetuximab to the EGFRvIII deletion mutant and its biological consequences in malignant glioma cells. Radiother Oncol 2009;92:3938

8. Okamoto I, Kenyon LC, Emlet DR, Mori T, Sasaki J, Hirosako S, et al. Expression of constitutively activated EGFRvIII in non-small cell lung cancer. Cancer Sci 2003;94:50-6

9. Sok JC, Coppelli FM, Thomas SM, Lango MN, Xi S, Hunt JL, et al. Mutant epidermal growth factor receptor (EGFRvIII) contributes to head and neck cancer growth and resistance to EGFR targeting. Clinical cancer research : an official journal of the American Association for Cancer Research 2006;12:5064-73

10. Golding SE, Morgan RN, Adams BR, Hawkins AJ, Povirk LF, Valerie K. Pro-survival AKT and ERK signaling from EGFR and mutant EGFRvIII enhances DNA double-strand break repair in human glioma cells. Cancer biology \& therapy 2009;8:730-8

11. Learn CA, Hartzell TL, Wikstrand CJ, Archer GE, Rich JN, Friedman AH, et al. Resistance to tyrosine kinase inhibition by mutant epidermal growth factor receptor variant III contributes to the neoplastic phenotype of glioblastoma multiforme. Clinical cancer research : an official journal of the American Association for Cancer Research 2004;10:3216-24

12. Theys J, Jutten B, Dubois L, Rouschop KM, Chiu RK, Li Y, et al. The deletion mutant EGFRvlll significantly contributes to stress resistance typical for the tumour microenvironment. Radiother Oncol 2009;92:399404

13. Weppler SA, Li Y, Dubois L, Lieuwes N, Jutten B, Lambin P, et al. Expression of EGFR variant vlll promotes both radiation resistance and hypoxia tolerance. Radiother Oncol 2007;83:333-9

14. Pelloski CE, Ballman KV, Furth AF, Zhang L, Lin E, Sulman EP, et al. Epidermal growth factor receptor variant III status defines clinically distinct subtypes of glioblastoma. Journal of clinical oncology : official journal of the American Society of Clinical Oncology 2007;25:2288-94

15. Gan HK, Kaye AH, Luwor RB. The EGFRvIII variant in glioblastoma multiforme. Journal of clinical neuroscience : official journal of the Neurosurgical Society of Australasia 2009;16:748-54

16. Del Vecchio CA, Jensen KC, Nitta RT, Shain AH, Giacomini CP, Wong AJ. Epidermal growth factor receptor variant III contributes to cancer stem cell phenotypes in invasive breast carcinoma. Cancer research 2012;72:2657-71

17. Li G, Mitra SS, Monje M, Henrich KN, Bangs CD, Nitta RT, et al. Expression of epidermal growth factor variant III (EGFRvIII) in pediatric diffuse intrinsic pontine gliomas. Journal of neuro-oncology 2012;108:395-402 
18. Liu XJ, Wu WT, Wu WH, Yin F, Ma SH, Qin JZ, et al. A minority subpopulation of CD133(+) /EGFRvIII(+) /EGFR(-) cells acquires stemness and contributes to gefitinib resistance. CNS neuroscience \& therapeutics 2013;19:494-502

19. Nagane M, Coufal F, Lin H, Bogler O, Cavenee WK, Huang HJ. A common mutant epidermal growth factor receptor confers enhanced tumorigenicity on human glioblastoma cells by increasing proliferation and reducing apoptosis. Cancer Res 1996;56:5079-86

20. Nishikawa R, Ji XD, Harmon RC, Lazar CS, Gill GN, Cavenee WK, et al. A mutant epidermal growth factor receptor common in human glioma confers enhanced tumorigenicity. Proc Natl Acad Sci U S A 1994;91:7727-31

21. Feng H, Hu B, Jarzynka MJ, Li Y, Keezer S, Johns TG, et al. Phosphorylation of dedicator of cytokinesis 1 (Dock180) at tyrosine residue Y722 by Src family kinases mediates EGFRvIll-driven glioblastoma tumorigenesis. Proc Natl Acad Sci U S A 2012;109:3018-23

22. Feng H, Hu B, Vuori K, Sarkaria JN, Furnari FB, Cavenee WK, et al. EGFRvIll stimulates glioma growth and invasion through PKA-dependent serine phosphorylation of Dock180. Oncogene 2014;33:2504-12

23. Dewhirst MW, Cao Y, Moeller B. Cycling hypoxia and free radicals regulate angiogenesis and radiotherapy response. Nat Rev Cancer 2008;8:425-37

24. Nordsmark M, Bentzen SM, Rudat V, Brizel D, Lartigau E, Stadler P, et al. Prognostic value of tumor oxygenation in 397 head and neck tumors after primary radiation therapy. An international multi-center study. Radiother Oncol 2005;77:18-24

25. Brizel DM, Sibley GS, Prosnitz LR, Scher RL, Dewhirst MW. Tumor hypoxia adversely affects the prognosis of carcinoma of the head and neck. Int J Radiat Oncol Biol Phys 1997;38:285-9

26. Hockel M, Schlenger K, Aral B, Mitze M, Schaffer U, Vaupel P. Association between tumor hypoxia and malignant progression in advanced cancer of the uterine cervix. Cancer Res 1996;56:4509-15

27. Wouters BG, van den Beucken T, Magagnin MG, Lambin P, Koumenis C. Targeting hypoxia tolerance in cancer. Drug Resist Updat 2004;7:25-40

28. Bigner SH, Humphrey PA, Wong AJ, Vogelstein B, Mark J, Friedman HS, et al. Characterization of the epidermal growth factor receptor in human glioma cell lines and xenografts. Cancer research 1990;50:8017-22

29. Kondo Y, Kanzawa T, Sawaya R, Kondo S. The role of autophagy in cancer development and response to therapy. Nat Rev Cancer 2005;5:726-34

30. Ogier-Denis E, Codogno P. Autophagy: a barrier or an adaptive response to cancer. Bba-Rev Cancer 2003;1603:113-28

31. Tracy K, Dibling BC, Spike BT, Knabb JR, Schumacker P, Macleod KF. BNIP3 is an RB/E2F target gene required for hypoxia-induced autophagy. Mol Cell Biol 2007;27:6229-42

32. Papandreou I, Lim AL, Laderoute K, Denko NC. Hypoxia signals autophagy in tumor cells via AMPK activity, independent of HIF-1, BNIP3, and BNIP3L. Cell Death Differ 2008

33. Rouschop KM, van den Beucken T, Dubois L, Niessen H, Bussink J, Savelkouls K, et al. The unfolded protein response protects human tumor cells during hypoxia through regulation of the autophagy genes MAP1LC3B and ATG5. J Clin Invest 2010;120:127-41

34. Rabinowitz JD, White E. Autophagy and metabolism. Science 2010;330:1344-8

35. Rouschop KM, Dubois L, Schaaf MB, van den Beucken T, Lieuwes N, Keulers TG, et al. Deregulation of cap-dependent mRNA translation increases tumour radiosensitivity through reduction of the hypoxic fraction. Radiother Oncol 2011;99:385-91

36. Sotelo J, Briceno E, Lopez-Gonzalez MA. Adding chloroquine to conventional treatment for glioblastoma multiforme: a randomized, double-blind, placebo-controlled trial. Ann Intern Med 2006;144:337-43

37. Struve N, Riedel M, Schulte A, Rieckmann T, Grob TJ, Gal A, et al. EGFRvIII does not affect radiosensitivity with or without gefitinib treatment in glioblastoma cells. Oncotarget 2015;6:33867-77

38. Wiederschain D, Wee S, Chen L, Loo A, Yang GZ, Huang A, et al. Single-vector inducible lentiviral RNAi system for oncology target validation. Cell Cycle 2009;8:498-504

39. Schaaf MB, Jutten B, Keulers TG, Savelkouls KG, Peeters HJ, van den Beucken T, et al. Canonical autophagy does not contribute to cellular radioresistance. Radiother Oncol 2015;114:406-12 
40. Schaaf MB, Cojocari D, Keulers TG, Jutten B, Starmans MH, de Jong MC, et al. The autophagy associated gene, ULK1, promotes tolerance to chronic and acute hypoxia. Radiotherapy and oncology: journal of the European Society for Therapeutic Radiology and Oncology 2013;108:529-34

41. N'Diaye EN, Kajihara KK, Hsieh I, Morisaki H, Debnath J, Brown EJ. PLIC proteins or ubiquilins regulate autophagy-dependent cell survival during nutrient starvation. EMBO Rep 2009;10:173-9

42. Rouschop KM, Dubois LJ, Keulers TG, van den Beucken T, Lambin P, Bussink J, et al. PERK/elF2alpha signaling protects therapy resistant hypoxic cells through induction of glutathione synthesis and protection against ROS. Proceedings of the National Academy of Sciences of the United States of America 2013;110:4622-7

43. Young SD, Marshall RS, Hill RP. Hypoxia induces DNA overreplication and enhances metastatic potential of murine tumor cells. Proc Natl Acad Sci U S A 1988;85:9533-7

44. Harris AL. Hypoxia--a key regulatory factor in tumour growth. Nat Rev Cancer 2002;2:38-47

45. Jin S, White E. Tumor suppression by autophagy through the management of metabolic stress. Autophagy 2008;4:563-6

46. Guo D, Hildebrandt IJ, Prins RM, Soto H, Mazzotta MM, Dang J, et al. The AMPK agonist AICAR inhibits the growth of EGFRvIII-expressing glioblastomas by inhibiting lipogenesis. Proceedings of the National Academy of Sciences of the United States of America 2009;106:12932-7

47. Maes H, Kuchnio A, Peric A, Moens S, Nys K, De Bock K, et al. Tumor Vessel Normalization by Chloroquine Independent of Autophagy. Cancer Cell 2014;26:190-206

48. Brown JM, Wouters BG. Apoptosis, p53, and tumor cell sensitivity to anticancer agents. Cancer research 1999;59:1391-9

49. Shinojima N, Tada K, Shiraishi S, Kamiryo T, Kochi M, Nakamura H, et al. Prognostic value of epidermal growth factor receptor in patients with glioblastoma multiforme. Cancer research 2003;63:6962-70

50. White E. Exploiting the bad eating habits of Ras-driven cancers. Genes Dev 2013;27:2065-71

51. Roudier E, Perrin A. Considering the role of pyruvate in tumor cells during hypoxia. Biochimica et biophysica acta 2009;1796:55-62

52. Rosenfeld MR, Ye X, Supko JG, Desideri S, Grossman SA, Brem S, et al. A phase I/II trial of hydroxychloroquine in conjunction with radiation therapy and concurrent and adjuvant temozolomide in patients with newly diagnosed glioblastoma multiforme. Autophagy 2014;10:1359-68

53. Guo JY, Chen HY, Mathew R, Fan J, Strohecker AM, Karsli-Uzunbas G, et al. Activated Ras requires autophagy to maintain oxidative metabolism and tumorigenesis. Genes Dev 2011;25:460-70

54. Levy JM, Thompson JC, Griesinger AM, Amani V, Donson AM, Birks DK, et al. Autophagy inhibition improves chemosensitivity in BRAF(V600E) brain tumors. Cancer Discov 2014;4:773-80

55. Mulcahy Levy JM, Zahedi S, Griesinger AM, Morin A, Davies KD, Aisner DL, et al. Autophagy inhibition overcomes multiple mechanisms of resistance to BRAF inhibition in brain tumors. Elife 2017;6 


\section{Supplementary figures}

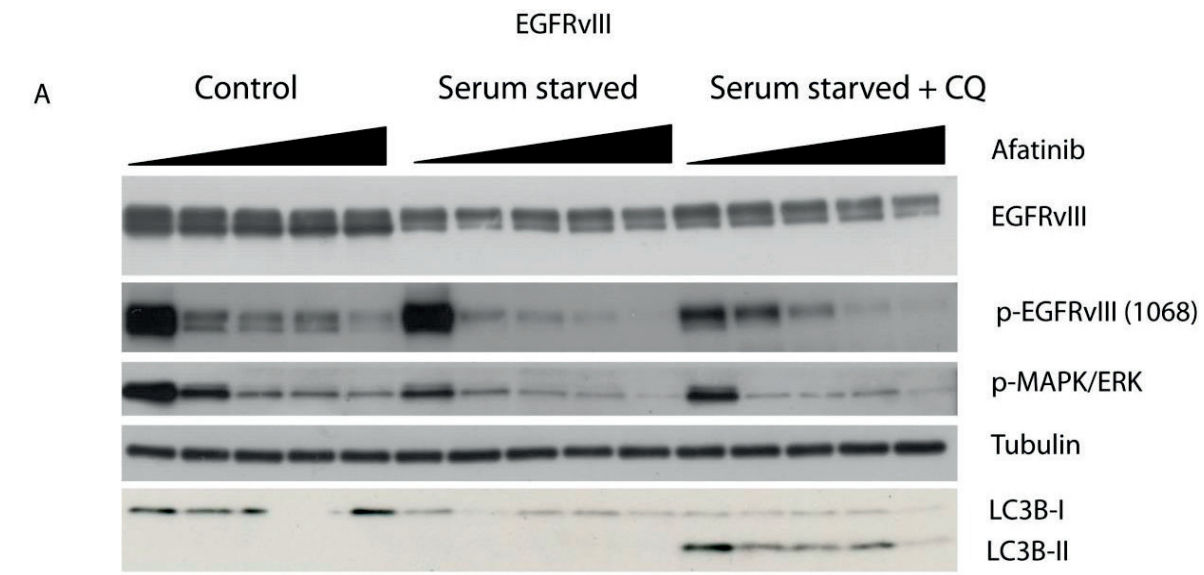

B

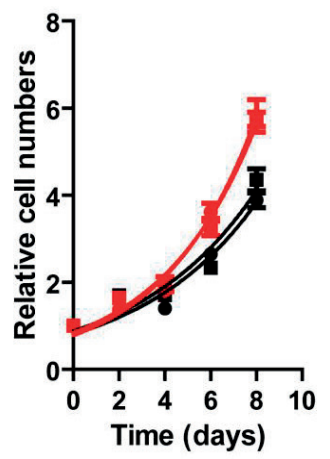

Supplementary figure 1
C

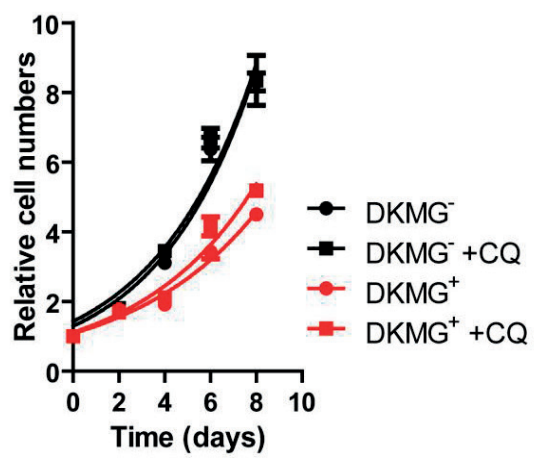


A

BrdU labeling index

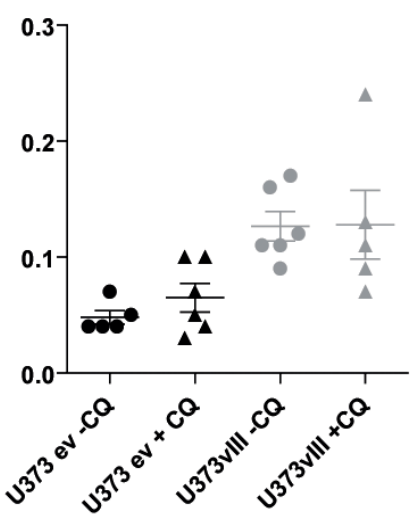

C

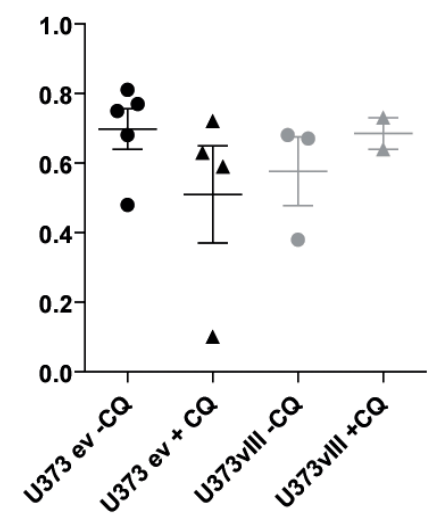

B Vessel density

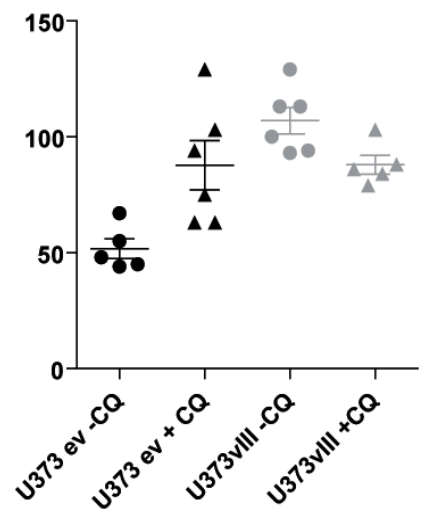

Supplementary figure 2

tumor growth delay

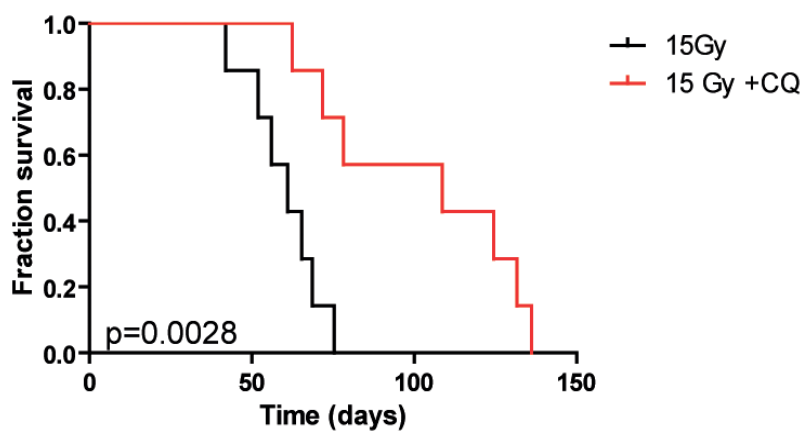

Supplementary figure 3 


\section{Chapter}

\section{ATG12 expression predicts tumour hypoxia and tumour control in HNSCC}

${ }^{1}$ Tom G. Keulers, ${ }^{2}$ Alexander Koch, ${ }^{1}$ Hanneke Peeters, ${ }^{1}$ Marike Van Gisbergen, ${ }^{1}$ Kim G.M. Savelkouls, ${ }^{3}$ Nicole Bitsch, ${ }^{4}$ Roel G. Wanders, ${ }^{5}$ Dennis Roop, ${ }^{6} J o h a n$ Bussink, ${ }^{2}$ Veerle Melotte, ${ }^{1}$ Kasper M.A. Rouschop

${ }^{1}$ Department of Radiotherapy, GROW - School for Oncology and Developmental Biology, Maastricht University Medical Centre+, Maastricht, The Netherlands 2Department of Pathology, GROW - School for Oncology and Developmental Biology, Maastricht University Medical Center, Maastricht, The Netherlands ${ }^{3}$ Cardiovascular Research Institute Maastricht, Universiteitssingel 50, 6229 ER, Maastricht, The Netherlands.

${ }^{4}$ MAASTRO Clinic, Maastricht, The Netherlands.

${ }^{5}$ Charles C. Gates Center for Regenerative Medicine, University of Colorado Denver, Aurora, CO, USA.

${ }^{6}$ Department of Radiation Oncology, Radboud University Medical Center, Nijmegen, The Netherlands. 


\section{Abstract}

Tumour hypoxia is a common feature of solid tumours and is associated with increased carcinogenesis, angiogenesis, resistance to therapy and metastasis. In agreement with these characteristics, clinical studies have shown prognostic value of tumour hypoxia, where high tumour hypoxia is associated with high mortality. Hence tumour hypoxia has been shown to be an independent prognostic factor for poor outcome, independent of treatment modality. To survive hypoxia, we previously showed that cells require activation of a lysosomal degradation pathway, autophagy. Here we show that expression of the essential autophagy protein autophagy related-12 (ATG12) is lost in 25\%-40\% of tumours of 2 independent head and neck cancer cohorts. Interestingly, patients with ATG12 low tumours display a decreased hypoxic fraction and thereby show improved local- and locoregional control and improved survival. Taken together we demonstrate that ATG12 expression is lost in a fraction of head and neck cancer tumours. Because ATG12 expression seems to predict tumour hypoxia and thereby tumour control, it is an interesting target to pursue as a predictive biomarker. 


\section{Introduction}

Autophagy is a cellular recycling mechanism that targets aged and cytotoxic content, such as aggregated proteins and damaged organelles, for degradation. Hence, during normal physiological and pathological conditions, autophagy is required to maintain cellular homeostasis. The process of autophagy commences with an extending doublemembrane structure, the phagophore, which (non-)selectively encapsulates the cargo. after closure, the vesicle (the autophagosomes) fuses with protease and hydrolase containing lysosomes, to expose its content to the degrading enzymes. The degradation products; amino acids, sugars and fatty acids are effluxed back to the cytoplasm and are used for biogenesis or metabolic purposes (1). Autophagy is orchestrated by a set of AuTophaGy-related (ATG) proteins, of which most are well-conserved from yeast to higher eukaryotes. Momentarily more than 38 ATG proteins have been identified and all exert unique functions (2). Due to its partial integration within the autophagosome and consequent turnover during autophagy, the yeast Atg8 orthologue, MAP1LC3B (LC3B), is frequently used as autophagy marker (3). Functionally, LC3B is essential in execution of autophagy as it allows membrane expansion and the fusion of extending membranes of the phagophore to fuse into a vesicle, the autophagosome (2).

Interaction of LC3B with the membrane requires conjugation of LC3B to phosphatidylethanolamine (PE), a process facilitated by the ATG8 and ATG12 ubiquitin-like conjugation systems (4). First, pro-LC3B is cleaved by the cysteine protease ATG4 to expose its $\mathrm{C}$-terminal glycine, followed by LC3B transfer to the E1 activating enzyme ATG7. LC3B is then further transferred from the LC3B-ATG7 complex to the conjugating enzyme ATG3 (E2). The ATG12-ATG5-ATG16L complex, which serves as the E3-like enzyme recruits the LC3B-ATG7 to the site of expansion. This ATG12-ATG5-ATG16L complex is formed by a second ubiquitin-like conjugation system, where ATG7 and ATG10 act as activating (E1) and conjugation enzyme (E2) respectively (5). The ATG12-ATG5-ATG16L complex is essential in elongation of the extending membrane (autophagophore) and deletion of either ATG5, ATG16L or ATG12 completely abolishes autophagosome formation (5-8).

In addition to its role in general autophagy execution, ATG12 mediates the selective degradation of mitochondria (mitophagy) by forming a covalent complex with ATG3 (9). Also non-autophagy mechanisms have been attributed to ATG12 function. For instance, ATG12 has pro-apoptotic properties by interacting with anti-apoptotic BCL2 family proteins via its BH3 only domain (Rubinstein, Eisenstein et al. 2011), independent of its conjugation to ATG5. Additionally, the ATG12-ATG3 complex promotes late endosome distribution and exosome biogenesis through interaction with the ESCRT protein ALIX (10).

The process of autophagy has a tumour suppressive role since it prevents aggregation and accumulation of cytotoxic agents, production of reactive oxygen species (ROS), inhibits inflammation and prevents genome instability (Mathew, Kongara et al. 2007) 
which potentially contribute to oncogenic transformation. In agreement, autophagydefective tumour cells display elevated genomic damage with stress, potentially caused by accumulation of protein-aggregates, damaged mitochondria and increased ROS (1113). Furthermore, defects in autophagy due to mono-allelic loss of beclin1 or oncogenic activation of the autophagy-suppressing PI-3kinase/mTOR pathway are common in human tumours (reviewed in (14)). For example, 40-75\% of human prostate, breast and ovarian cancers display mono-allelic loss of beclin1 (15-18). Also in mice, targeted deletion of a single beclin1 allele predisposes to spontaneous tumour development $(19,20)$, indicating that autophagy is a tumour suppressive mechanism. Also other defects in autophagy are associated with tumorigenesis, ATG5 and ATG7 deficient mice are shown to spontaneously develop hepatic neoplasms (21).

In the tumour microenvironment (TME), tumour cells can be exposed to a multitude of stressors, including nutrient deprivation and hypoxia. Hypoxia is mainly caused by the (temporary) occlusion of blood vessels (acute hypoxia) or by limited diffusion due to insufficient vascularisation (chronic hypoxia) to meet the metabolic requirements of the tumour. From a clinical point of view, means of reducing the hypoxic fraction of tumours is highly desired since low oxygenation of tumours is associated with poor outcome in multiple cancer types (22), independent of treatment modality (23). In HNSCC, where a meta-analysis indicated that the degree of hypoxia is the most significant factor explaining variability in survival (24), the evidence is strongest. The observed effect on local control is most likely caused by the resistance of hypoxic cells to both chemo- and radiotherapy. Additionally there appears to be an association between hypoxia and the occurrence of metastasis (25) and it has been proposed that tumour hypoxia contributes to malignancy e.g. through activation of epithelial to mesenchymal transition(2629). Importantly, a meta-analysis in HNSCC demonstrated therapeutic benefit of hypoxia modification (30). Autophagy mainly localizes in hypoxic areas and contributes to the survival of hypoxic cancer cells and thereby contributes to regrowth of the tumour and drives tumour cells into a more resistant, invasive and metastatic phenotype. Pharmacological and genetic inhibition of autophagy results in decreased tumour hypoxia and contributes to improved tumour control and treatment efficacy (31).

In this study we show that $40 \%$ of head and neck cancer patients have a single copy deletion of the ATG12 gene and approximately 25\% of the tumours fail to express ATG12. Interestingly, patients with ATG12 deficient tumours display increased locoregional control and improved survival after therapy. 


\section{Materials and methods}

\section{Cell culture}

HT29 (colorectal adenocarcinoma) and UTSCC5 (established from tumours from the tongue by Dr. Reidar Grenman, University of Turku, Finland (32). cells were cultured in DMEM (LONZA) and DMEM supplemented with 0.1mM non-essential amino acids (Sigma Aldrich) respectively supplemented with 10\% FCS. Hypoxia exposure (16-20 hours over night) was done using a modular atmosphere controlled system (Don Whitley Scientific). Doxycycline inducible knockdown of ATG12 was achieved through lentiviral transduction and expression of shRNA from pTRIPZ backbones TGATGAAGTCAATGAGTCC (\#1) and AAACAACTGTTCTGAGGCC (\#2). Knockdown was induced 6 days prior to experiment. MEFs were isolated as described elsewhere (33).

\section{Clonogenic survival assays}

Knockdown was induced with doxycycline 7 days before experiments. Cells were seeded and exposed to hypoxia $\left(0.2 \% \mathrm{O}_{2}\right)$ for $0,24,48,72$ or 96 hours using a modular atmosphere controlled system (Don Whitley Scientific). After exposure, medium was changed without doxycycline and incubated for $+/-10$ days to allow colony formation. Colonies were color-fixed ( $0.4 \%$ methylene blue in $70 \% \mathrm{EtOH}$ ) (Sigma Aldrich, M4159) and counted manually.

\section{Mitochondrial respiration experiments}

Oxygen consumption of UTSCC5 (80.000 cells/well) was measured using the XFe 24 extracellular flux analyzer (Agilent Technologies, Santa Clara, California). Cells were seeded in DMEM containing L-glutamine (Gibco, Paisley, Scotland, UK), supplemented with $0.9 \mathrm{~g} / \mathrm{L}$ galactose (Sigma-Aldrich, St. Louis, Missouri), MEM Non-essential amine acids solution (Gibco, Scotland, UK), and 10\% FCS (Sigma-Aldrich, St. Louis, Missouri) and incubated overnight in an incubator containing $5 \% \mathrm{CO}_{2}$ at $37^{\circ} \mathrm{C}$. Basal oxygen consumption rate was measured 1 hour after replacing the medium to Seahorse XF Base Medium (Agilent Technologies) containing $0.9 \mathrm{~g} / \mathrm{L}$ galactose (Sigma-Aldrich, Saint Louis, Missouri) and L-glutamine (Gibco, Paisley, Scotland, UK) and the cells were equilibrated in an $\mathrm{CO}_{2}$ free incubator at $37^{\circ} \mathrm{C}$. Data were corrected for confluency of the wells.

\section{Quantitative $P C R$}

RNA extraction was done using Nucleospin RNA kit (Macherey-Nagel) and cDNA was prepared using I-script (Bio-Rad). Patient RNA was extracted using TRI reagent (Sigma Aldrich) according to the manufacturers manual Real-time PCR was done using ABI7500 real-time PCR (Applied Biosystems). mRNA abundance was measured using SYBR green (Thermos Fisher). Abundance of each gene was normalized to $18 \mathrm{~s}$. 


\begin{tabular}{lll}
\hline Target & Forward & reverse \\
\hline ATG12 set 1 & AGGTCTGTAGTCGCGGAGAA & CGGGAACACCAAGTTTCACT \\
ATG12 set 2 & CCCAGACCAAGAAGTTGGAA & TGATGCTTGTGGCAAGAGAC \\
ATG12 set 3 & CTTACGGATGTCTCCCCAGA & AGTGAGGACTTTGGGTGTGG \\
LC3B & AACGGGCTGTGTGAGAAAC & GACTCCGGCGGAAGCAT \\
VEGF & TCCGGGCTCGGTGATTTA & GCAAGCCAGACAGGAAAAG \\
ATG5 & GACCTTCAGTGGTCCGGTAA & GAT CCG AGG GCC TCA CTA AAC \\
\hline
\end{tabular}

\section{Immunoblotting.}

Cells were lysed in RIPA buffer (50mM Hepes-KOH, pH 7.5, 150mM KCL, 1mM EDTA, $2 \mathrm{mM}$ mercaptoethanol, $0.2 \%$ Tween-29 with protease inhibitor (Roche) and sonicated 3 $x 10$ seconds at $10 \mathrm{MHz}$. Samples were separated by SDS-PAGE and transferred to PVDF membranes. ATG5 (1:1000, D5F5U, cell signaling) ATG12 (1:1000, D88H11, cell signaling) LC3B (1:1000, PM036, MBL) Actin (1:200.000, MD bioscience) antibodies were used as primary antibodies. Antibodies were visualized using HRP linked secondary antibodies (anti-Rabbit, anti-mouse, 1:2000, Cell signaling). Super Signal west pico chemiluminescent substrate (pierce biotechnology, Thermo Scientific) was used for detection.

\section{Tumour models}

To study the role of ATG12 on tumour growth and its impact on radiotherapy, doxycycline inducible shATG12-UTSCC5 cells were injected subcutaneously in the flanks of NMRInu/nu mice (1.0E6 cells/50ul matrigel (Corning)). Doxycycline was administered via the drinking water ( $2 \mathrm{~g} / \mathrm{L}, 5 \%$ sucrose). Tumour size was assessed by caliper measurements (34). Animals were injected with BrdU (30 mg/ kg, Sigma Aldrich) and pimonidazole (60 mg/ kg, Hypoxyprobe) intraperitoneally 1 hour prior to killing. For irradiation experiments; after reaching $150 \mathrm{~mm}^{3}$ in tumour size, animals were anesthetized (ketamine/xylazine) and tumours were irradiated with a single, tumour-specific, dose of 10Gy. All animal experiments were conducted in accordance with national guidelines and approved by the animal ethics committee Maastricht University.

\section{Patient cohorts}

The head and neck cancer cohort was obtained from The Cancer Genome Atlas (https://xenabrowser.net/heatmap) TCGA Head and neck cancer HNSC, n=479). HPV positive tumours were excluded from the analysis. Data was sorted on ATG12 expression and split in quartiles ( $1^{\text {st }}$ (low ATG12) and $2^{\text {nd }}-4^{\text {th }}$ quartile (high ATG12 expression). Outcome was evaluated by overall survival. Curve comparison was done using the log-rank test (Graphpad prism software). Copy number was also extracted from the database.

RADPLAT (35), $n=86$ ) and ARCON (36)) (n=17) cohorts were combined. ATG12 expression was determined by qPCR (ARCON) and normalized to $18 \mathrm{~S}$ expression. Data was 
sorted and split in quartiles; $1^{\text {st }}$ (low ATG12) and $2^{\text {nd }}-4^{\text {th }}$ quartile (high ATG12 expression). Local control and loco-regional control was used to evaluate outcome.

\section{Immunohistochemistry and image processing}

Frozen acetone fixed cross sections were stained using ATG12 (D88H11, cell signaling) Pimonidazole (Chemicon), 9F1 Rat mAb to mouse endothelium, (Radboud University Nijmegen, Dept. of Pathology) as described previously (34). Slides were quantified by computerized digital image processing system using fluorescent microscope with a computer-controlled motorized stepping stage, equipped with a high-res intensified solid-state camera (Axioskop; Zeis). Necrosis was examined morphologically by H\&E stainings. Hypoxic fraction, necrosis and (perfused) vascular densities are indicated fraction of the total tumour volume.

\section{Generation of conditional ATG12 knockout mice}

To Allow conditional deletion of Atg12 exon2 (ENSMUSE00000249693), Atg12 recombined embryonic cell clones (Atg12 HEPD0670_6_A10, HEPD0670_6_D12 and HEPD0670_6_G09, EUCOMM, Germany) were injected into C57BL/6J blastocysts and re-implanted into OF1 pseudo pregnant females and allowed to develop to term (Genoway, France). Chimeras are cross bred with ubiquitous Flp-recombinase expressing deleter mice to mediate deletion of FRT-flanked regions in the Atg12 recombined locus containing lacZ reporter cassette and neomycin positive selection cassette. Recombination of FRT sites al is used to select for heterozygous conditional knockout mice as determined by PCR and southern blot. Homozygous Atg12 conditional knockout mice are bred with mice expressing a Cre-recombinase and human truncated progesterone receptor fusion protein (Cre*PR1) under control of a bovine Keratin5 promotor to allow transgene expression in the epidermis and head and neck epithelia (37). To induce translocation of Cre*PR 1 to the nucleus and recombination of LoxP sites to knockout the Atg12 exon 2, the progesterone antagonist RU486 is applied in to oral cavity $(2 \mu \mathrm{g} / \mu \mathrm{l}$ in sesame oil). Mice with floxed SMAD4 genes were purchased from the Jackson Laboratories (Smad4tm2.1Cxd/J, Jackson laboratories),

\section{Results}

\section{ATG12 expression is lost in a subpopulation of HNSCC}

Defects in autophagy can lead to neoplastic growth and contribute to tumorigenesis and tumour progression (reviewed in (14)). Surveying RNA expression levels of autophagyassociated genes by PCR in a panel of 17 HNSCC patients we observed lack of expression of ATG12 mRNA in 7 of 17 patients (fig 1A). Subsequently, ATG12 RNA abundance was 
measured by qPCR, including the autophagy-related genes ATG5 and LC3B and the vascular endothelial growth factor VEGF. Although not as binary as in conventional PCR analysis, ATG12 mRNA abundance is lower in 5 out of 17 patients (29\%). ATG5, LC3B and VEGF expression did not follow a comparable pattern in these patients (fig 1B).

To determine if lower ATG12 mRNA expression is due to absence of gene expression or decreased ability of the primers to recognize ATG12 mRNA due to gene truncation or mutations, 3 independent primer pairs targeting the 3'UTR, coding sequence and 5' UTR were used. The identical pattern of the 3 independent primer pairs, indicate loss of full transcript expression (suppl fig 1). Reduced gene expression can be the result of several mechanisms such as deletion of the gene region, gene silencing by promoter methylation or mutations. To determine the cause of reduced ATG12 gene expression, we analysed the HPV-negative HNSCC cohort of The Cancer Genome Atlas (TCGA, cancergenome.nih.gov, $n=479$ ). Of 429 HNSCC (epi-) genomic information is available. Of these 429 tumours, $2(0.4 \%)$ tumours displayed homozygous deletion of the ATG12 gene, 174 (40\%) displayed single copy deletion (fig 1C), suggesting that deletion events contribute to loss of ATG12 expression in HNSCC (fig 1D). Compared to healthy controls, no differences in ATG12 gene-methylation were observed (data not shown).

\section{ATG12 loss is associated with decreased tumour hypoxia in HNSCC}

Autophagy is required to maintain a viable hypoxic fraction and sustain survival during stress in the tumour microenvironment. We showed before that targeting autophagy decreases the hypoxic fraction of the tumour (31). Since ATG12 is required for the general execution of autophagy we characterized the hypoxic fraction and other TME associated parameters (vasculature) of a panel of 51 patients who received the hypoxia marker pimonidazole (ARCON study Nijmegen (36)). ATG12 expression was assessed by immunohistochemical staining, scored manually, and classified as ATG12 negative and positive tumours (fig 1E. Tumour biopsies without or only few $(<1 \%)$ ATG12 positive cells were grouped as ATG12 low. Strikingly, ATG12 $2^{\text {low }}(n=14)$ expressing tumours display no or strongly decreased hypoxic fraction, compared to ATG12 expressing tumours ( $n=37$ ) (fig 1F). Increased delivery of oxygen could be the underlying mechanism, but tumour vasculature (number and size of vessels) of ATG12 low and ATG12 ${ }^{\text {high }}$ expressing tumours were comparable (fig $1 \mathrm{G}$ and $\mathrm{H}$ ). These data suggest that ATG12 negative tumours consume less oxygen, cannot sustain a viable hypoxic fraction or ATG12 is a hypoxia regulated gene. To test if the lack if ATG12 expression is causally related to the absence of oxygen, ATG12 expression of cells exposed to hypoxia was assessed by qPCR. ATG12 mRNA abundance is not regulated by hypoxia in 5 out of 7 cell lines tested (fig 1 l and J). These data suggest that the lack of hypoxia is due to loss of ATG12 and not vice versa. This is further supported by the fact that in patient tumours, ATG12 is expressed throughout the tumour and is not restricted to hypoxic areas only (fig $1 \mathrm{E}$ ). 
A

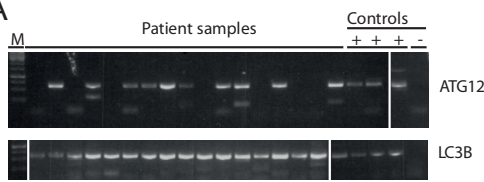

C

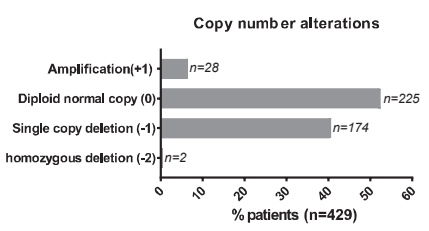

E
B

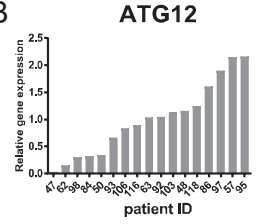

VEGF

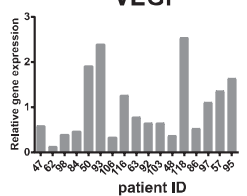

ATG5

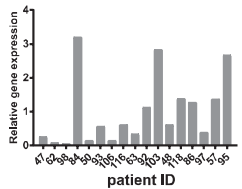

MAP1LC3B

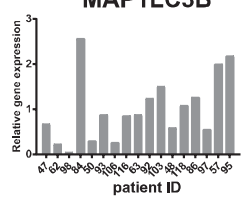

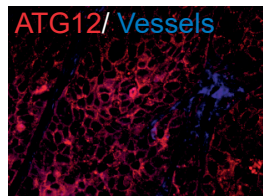
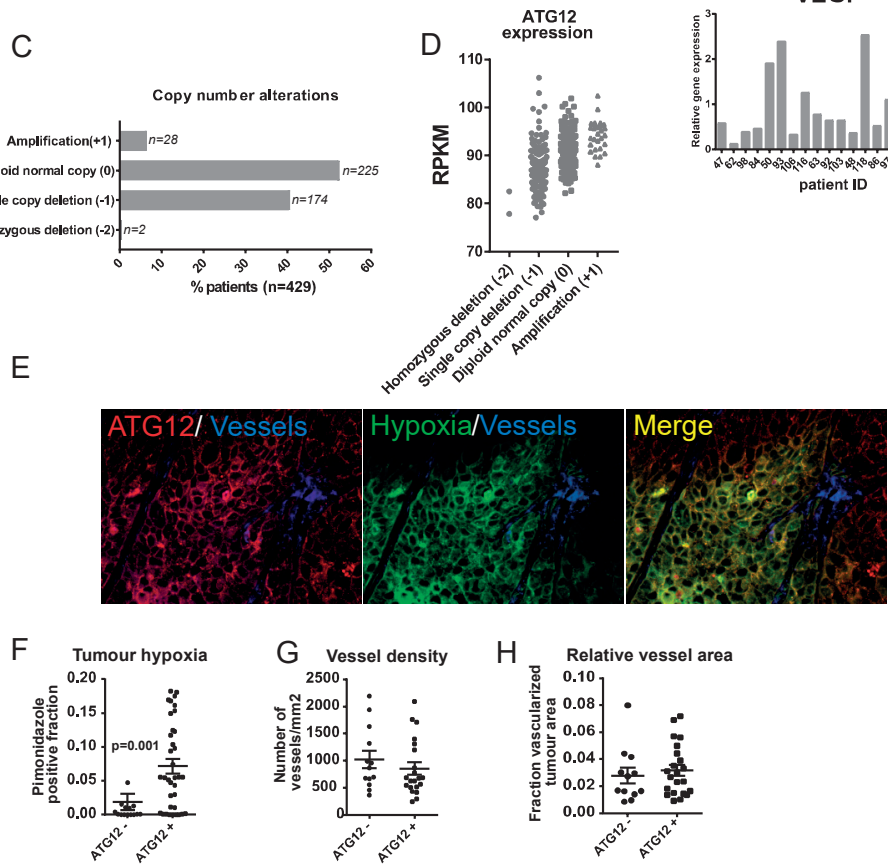

I

ATG12
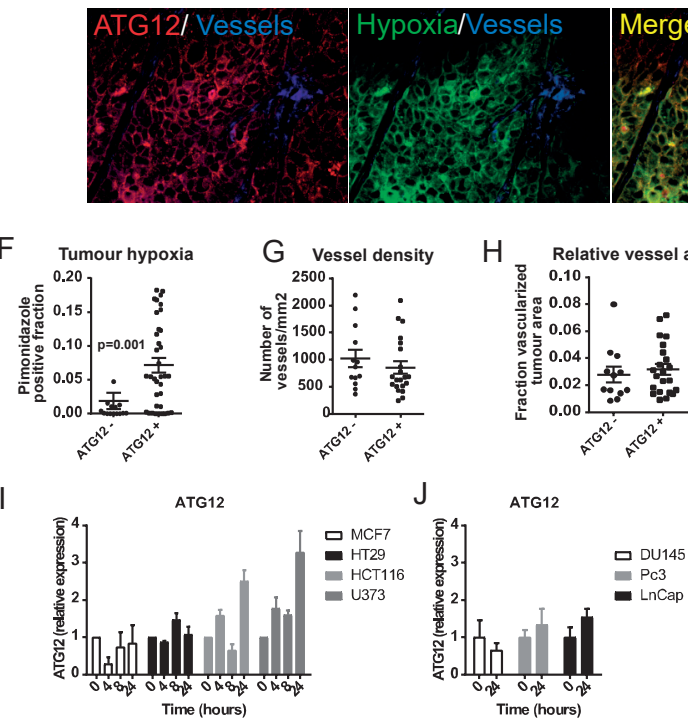

Figure 1: ATG12 expression is lost in a subpopulation of HNSCC

(A) Conventional PCR shows lack of ATG12 mRNA expression in 7 of 17 patients. (B) Quantitative real-time PCR confirms decreased ATG12 mRNA abundance in 5 of 17 patients. ATG5, LC3B and VEGF do not follow this pattern. (C) $40 \%$ of tumours form the TCGA HNSCC cohort show a single copy deletion of the ATG12 gene. (D) ATG12 gene expression of tumours from the TCGA HNSCC cohort as determined by RNA-seq. RPKM = Reads per kilo base per million mapped reads. (E) Immunohistochemical staining of ATG12 (Red) and hypoxia (pimonidazole, green). (F) Loss of ATG12 expression is associated with decreased tumour hypoxia in HNSCC biopsies. (G) ATG12 negative (ATG12-) and ATG12 expressing tumours (ATG12+) have similar vessel densities and $\mathbf{( H )}$ relative vessel areas (vessel size). (I and $\mathbf{J})$ Real-time quantitative PCR analysis of ATG12 mRNA expression in 7 cancer cell lines exposed to severe hypoxia $\left(0.02 \% \mathrm{O}_{2}\right)$. 
Loss of ATG12 expression correlates with improved prognosis in HNSCC patients

Tumour hypoxia is a negative prognostic factor associated with poor treatment outcome (24) and modifying tumour hypoxia results in improved outcome after therapy (39). Previously we showed that genetic and pharmacological inhibition of autophagy reduces tumour hypoxia and increases efficacy of several treatment modalities including radiotherapy $(31,40)$. Since ATG12-negative tumours display no or strongly decreased hypoxic fraction, we evaluated the effect of ATG12 deficiency on local and locoregional control, all treated in combination with radiotherapy. In a cohort of 103 stage 3 and $4 \mathrm{H} \& \mathrm{~N}$ cancer patients, ATG12 mRNA expression was determined by qPCR and divided into quartiles (table1). Patients with ATG12 low $\left(1^{\text {st }}\right.$ quartile) tumours display increased local and loco-regional control (fig 2A) compared to patients with ATG12 ${ }^{\text {high }}$ $\left(2^{\text {nd }}-4^{\text {th }}\right.$ quartile) tumours.

\section{A}
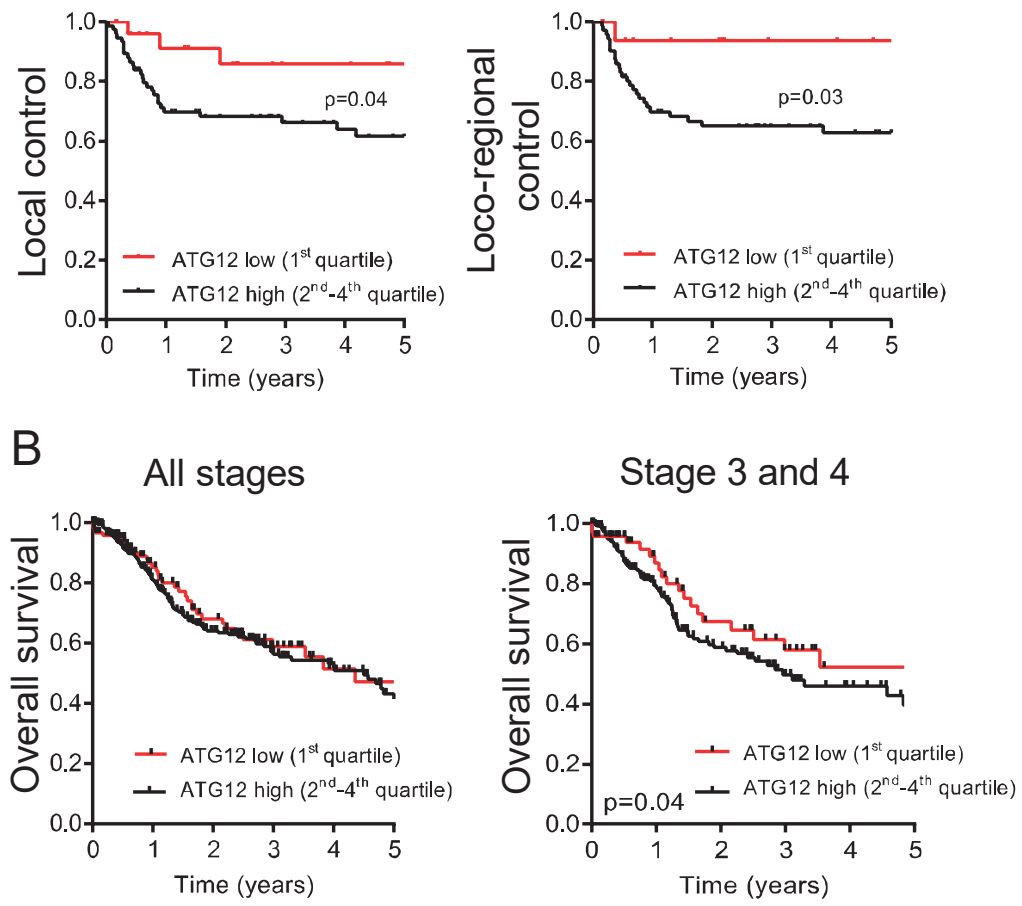

Figure 2: Loss of ATG12 expression correlates with improved prognosis in HNSCC patients

(A) Patients with ATG12 $2^{\text {low }}$ tumours (red line) show increased local and loco-regional control compared to patients with ATG12 ${ }^{\text {high }}$ tumours (Black line). ATG12 expression was determined by RT-qPCR and divided in quartiles (Curve comparison was done using the log-rank test, $p=0.04$ and 0.03 respectively). (B) Patients with ATG12 low tumours show improved overall survival in the TCGA HNSCC cohort as compared to patients with ATG12 ${ }^{\text {high }}$ tumours. (HPV negative, $n=479$. Curve comparison was done using the log-rank test, $p=0.04$ ) 
These findings were validated in the HNSCC patient cohort (HPV-negative, $n=479$ ) (table 2) of the The Cancer Genome Atlas (TCGA, cancergenome.nih.gov). ATG12 mRNA expression data and overall survival was extracted from the database and the ATG12 low group ( $1^{\text {st }}$ quartile) was compared to ATG $12^{\text {high }}\left(2^{\text {nd }}-4^{\text {th }}\right.$ quartile). Interestingly, ATG12 expression did not have any prognostic value on overall survival of all patients (stage 1 to 4) (fig 2B). However, similar as in fig $2 \mathrm{~A}$, in with more progressed tumours (stage 3 and 4) ATG12 low provides improved overall survival (fig 2B). Taken together these data indicate that low ATG12 mRNA expression is associated with increased local and locoregional control and thereby contributes to increased overall survival in H\&N cancer patients.

\section{Loss of ATG12 expression contributes to improved tumour control.}

ATG12 expression is decreased in a subpopulation of $29 \%$ of $H \& N$ cancer patients. Interestingly, patients with low ATG12 expressing tumours display improved treatment outcome and increased overall survival rates. ATG12 is located on chromosome 5.q, a chromosomal region frequently deleted in head and neck cancer (41). To determine if the observed effects in patients are due to the loss of ATG12 expression and not to the loss of other genes located in the same chromosomal region, we engineered the HNSCC cell line, UTSCC5 with ATG12 inducible (doxycycline) knockdown. Loss iof ATG12-ATG5 complex formation and the lack of LC3B-conversion, indicate functional loss of ATG12 in these cells after doxycycline exposure (fig 3A). To monitor in vivo effects, these cells were subcutaneously implanted in the flanks of NMRI ${ }^{\text {nu/nu }}$ mice. No differences in growth rates were observed (fig $3 B$, and C). Surprisingly, as determined by pimonidazole immunohistochemistry, ATG12 loss does not change the hypoxic fraction in UTSCC5 tumours (fig 3D). However, further analysis indicated that ATG12 deficient tumours display an increased necrotic phenotype of the total tumour area compared to control tumours (fig 3E), suggesting that ATG12 deficient cells have a survival disadvantage when exposed to stresses in the tumour microenvironment. In addition, no differences in vessel density of ATG12 knockdown tumours was observed (fig 3F). However, the number of perfused vessels is significantly decreased in ATG12 knockdown tumours (fig 3G).

In patients, loss in ATG12 expression contributed to improved outcome after therapy (fig 2). To test if this loss is the causal factor of this observation, UTSCC5 tumours were grown with wild-type characteristics until they reached $\pm 150 \mathrm{~mm}^{3}$. Once the tumours reached $150 \mathrm{~mm}^{3}$, doxycycline was administered through the drinking water to induce knockdown of ATG12, followed by tumour specific irradiation (10Gy single dose). As expected, tumour irradiation of control tumours resulted in growth delay (fig $3 \mathrm{H}$ ). Regrowth, as expressed in times to reach $4 x$ the irradiated volume, is significantly increased in ATG12 knockdown xenografts (fig 3I). 7 of the 10 ATG12-deficient tumours completely regressed and were still not visible at the time where all control tumours 
had reached their endpoint (fig 3I). These data indicate that, in line with patient data, lost ATG12 expression indeed contributes to improved treatment outcome.
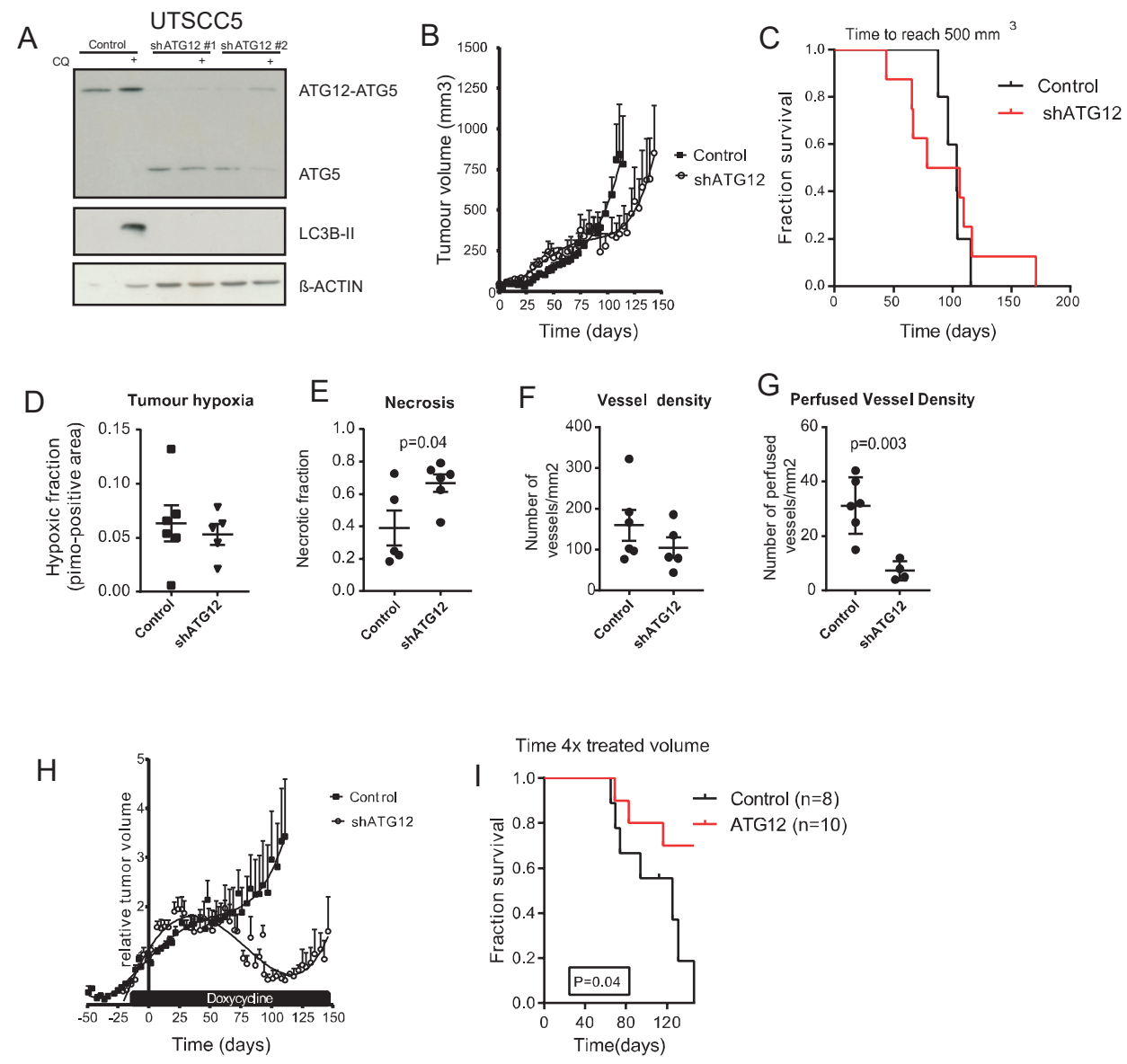

Figure 3: Loss of ATG12 expression contributes to improved tumour control.

(A) Immunoblot of UTSCC5 ATG12 knockdown cells expressing 2 independent ATG12 targeting hairpins. (B) Growth curves of UTSCC5 control ( $n=5$, open circles) and inducible ATG12 knockdown ( $n=8$, filled squares) xenografts. (C) Kaplan-meier curve of tumours reaching $500 \mathrm{~mm}^{3}$. (D) Hypoxic fraction of UTSCC5 xenografts was determined using pimonidazole immunohistochemistry (error bars \pm SEM). (E) Tumour necrosis of UTSCC5 xenografts was examined morphologically by H\&E staining (error bars \pm SEM, $p=0.04$, students' t-test). (F) Vessel density as determined by 9F1 immunohistochemistry (error bars \pm SEM). (G) Perfused vessel density is decreased in ATG12 knockdown xenografts (error bars \pm SEM, $p=0.003$, Students's t-test). (H) Established tumours were irradiated (10Gy) when reached $150 \mathrm{~mm}^{3}$. Doxycycline was administered through the drinking water 7 days prior to the treatment to induce expressing of the hairpin. ATG12 knockdown xenografts (open circles, $n=10$, error bars \pm SEM) show a tumour regrowth delay compared to controls (filled squares, $n=10$, error bars \pm SEM). (I) Kaplan-meier curve of xenografts reaching $4 x$ the irradiated volume 


\section{ATG12 is not required for survival during hypoxia}

Previously we showed that genetic and pharmacological inhibition of autophagy sensitizes cells to hypoxia $(31,42,43)$. The absence of tumour hypoxia in ATG12 low tumours in patients would suggest that ATG12 deficient cells are less resistant to hypoxia than ATG12 proficient cells. Therefore we tested if ATG12 is required for survival during hypoxia. For survival studies we cultured UTSCC5 and HT29 ATG12 knockdown cells and ATG12\% MEF (fig 4A) and exposed them to severe hypoxia $\left(<0.02 \% \mathrm{O}_{2}, 16 \mathrm{~h}\right.$ ) followed by colony formation assessment. Interestingly, these studies indicate that ATG12 is not required for survival during severe hypoxia (fig 4B). Unexpectedly, ATG12 knockdown led to increased survival in UTSCC5 and MEF cells. These findings are in line with previous findings that show that silencing ATG12 improves cell survival after stimulation with apoptosis inducers (44). Taken together, these data suggest that the role of ATG12 in pro-death mechanisms is stronger than pro-survival mechanisms like autophagy.

Survival studies with UTSCC5 cells show that ATG12 is not required for cell survival during hypoxia, which implies that intrinsically ATG12 deficient cells are in fact able to sustain a viable hypoxic fraction. Tumour cells typically have an altered metabolism associated with increased oxygen consumption that contributes to hypoxia. To determine if the decreased hypoxic fraction observed in patients can be explained by decreased oxygen consumption, we determined the oxygen consumption by measuring the oxygen consumption rate (OCR) of ATG12 knockdown cells. Interestingly, ATG12 deficient UTSCC5 cells display no differences in OCR (fig 4C).

\section{Deletion of Atg12 contributes to tumorigenesis}

The high rate of ATG12 deletions in head and neck cancer patients prompted us to investigate if the loss of ATG12 expression plays a causal role in tumorigenesis. To test this, we engineered a conditional Atg12 knockout tumour model specific for the head and neck region. In this model, Cre-recombinase is fused to a truncated progesterone receptor (*PR1) which can be activated by oral administration of the progesterone antagonist RU486 (Fig 5A). The Cre*PR1 fusion protein is driven by the keratin5 (K5) promoter, allowing specific gene expression in the epidermis and head and neck epithelia (including the tongue, oesophagus, forestomach and oral cavity) (45). Upon RU486 administration in the oral cavity, Cre*PR1 is translocated to the nucleus, were it specifically recombines lox sites where after $A \operatorname{tg} 12^{\mathrm{fl} / \mathrm{fl}}$ is permanently deleted (Fig 5B). Smad $4^{f / / f l}$ is shown to be a driver for HNSCC formation and is taken as a positive control (46). Weekly inspection of the oral cavity and at the point of termination of the experiment (week 104) revealed an increase in suspected macroscopic aberrations in Atg12-/(54\%), Smad4\% (41\%) and Atg12\%/Smad4\% (45\%) compared to the control group (15\%) (Fig 5C). Suspected lesions are characterized by abnormal appearance of the thymus, salivary glands or spots on the tongue and buccal mucosa. However, pathological histological confirmation is required. 
A

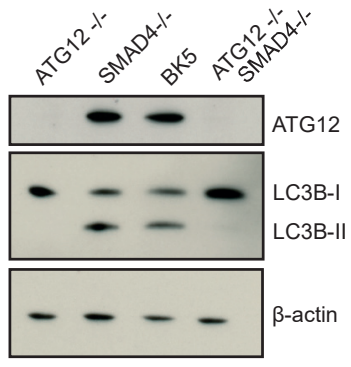

HT29

Control shATG12\#1shATG12 \#2

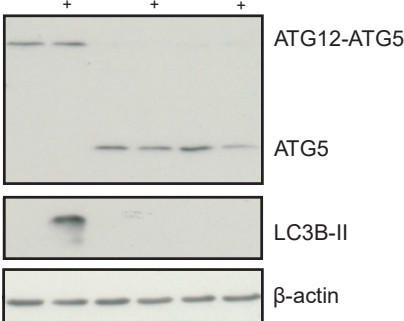

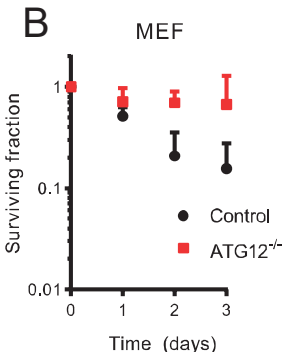

UTSCC5

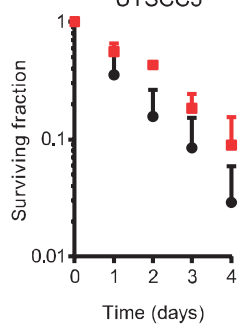

HT29

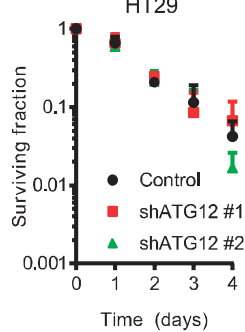

OCR

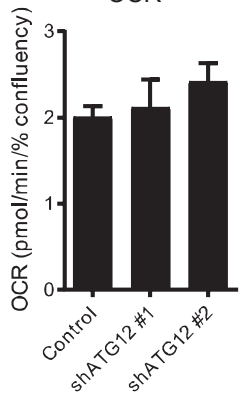

Figure 4: ATG12 is not required for survival during hypoxia

(A) Immunoblots of MEFs isolated from ATG12 $2^{\mathrm{fl} / \mathrm{fl}}, \mathrm{SMAD} 4^{\mathrm{fl} / \mathrm{fl}}$ and ATG12/SMAD4 $4^{\mathrm{fl} / f 1}$ mice show functional knockout of ATG12. BK5= Bovine keratin 5 promoter and used as control. HT29 expressing shATG12 show functional knockdown of ATG12. (B) Clonogenic survival of ATG12\% MEF and UTSCC5 and HT29 cells expressing 2 independent ATG12 targeting hairpins ( $n=3$, error bars \pm SEM). (C) Oxygen consumption rate of UTSCC5 control and shATG12 cells

A

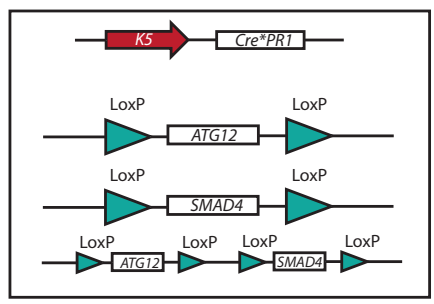

B

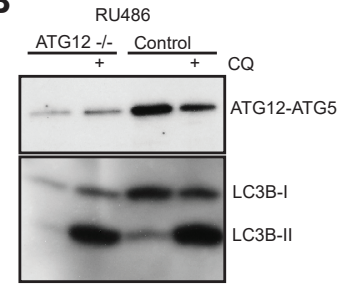

C spontaneous tumours

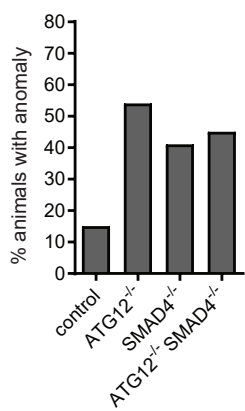

Figure 5 Deletion of Atg1 2 contributes to tumorigenesis

(A) Strategy to knock out ATG1 2 na SMAD4 in the head and neck rgion. $\mathrm{K} 5=$ keratin 5 promoter, $\mathrm{Cre}=\mathrm{cre}-$ recombinase, ${ }^{*} P R 1$ = truncated prgesterone receptor. LoxP= Lox recombination site. (B) Immunoblots of ATG1 $2^{\text {fl }}$ MEFs. RU4 86 was administered for Cre-mediated recombinatio6). Number of mice with anomalies in the head and neck region. (control $n=26, \operatorname{ATG1} n z 26, \operatorname{SMAD} 4 \mathrm{~h}=2$ 6ATG1 $2 / \mathrm{SMAD}^{-1} \mathrm{n}=26$ ) 


\section{Discussion}

In this study we show that expression of the essential autophagy gene ATG12 is lost in a subpopulation of HPV-negative HNSCC. Interestingly, patients with ATG12 deficient and autophagy-defective tumours display increased local and loco-regional control and improved overall survival in stage 3 and 4 of the disease. In solid tumours, tumour hypoxia is an important component of treatment efficacy as it contributes to resistance to several treatment modalities such as radiotherapy. Analysis of the TME revealed that ATG12 deficient tumours lack the presence of hypoxic regions, suggesting that these tumours cannot maintain a viable hypoxic fraction due to defective autophagy. The lack of a hypoxic fraction seems to be the causal factor of improved therapy efficacy in these patients. Several studies and ongoing clinical trials are based on the findings that autophagy inhibition contributes to improved therapy outcome. In this respect it is rather logically that autophagy defective tumours respond better to therapies. However, controlled in vivo studies with ATG12 knockdown xenografts only seem to partially repeat those findings. In line with the observed effects in patients, UTSCC5 ATG12 knockdown xenografts do respond better to radiotherapy than their controls. However, this effect seems independent of the levels of hypoxia in these tumours as hypoxic fractions do not differ between control and knockdown groups. However, due to the necrotic phenotype of ATG12 knockdown tumours (median is 72\% necrotic fraction in ATG12 knockdown tumours), the absolute amount of viable hypoxic cells, which are able to repopulate the tumour after therapy, are less than their control counterparts in size-matched tumours. The necrotic phenotype of ATG12 knockdown xenografts indicates that, in line with patient data, these tumours are less resistant to stresses in the TME. However, in vitro survival studies show that ATG12 is not required for survival during hypoxia. Typically, autophagy inhibition sensitizes cells to hypoxia resulting in decreased cell survival (31). That inhibition of autophagy by silencing of ATG12 does not so, possibly lies in proapoptotic properties of ATG12 by interacting with anti-apoptotic BCL2 family members to promote apoptosis by cytochrome c release (Rubinstein, Eisenstein et al. 2011). This suggests that at this conversion point between autophagy and apoptosis, the role of ATG12 is probably more pro-apoptotic. Prolonged exposure to stress in the TME will eventually lead to necrotic cell death. Unexpectedly, survival studies show that silencing ATG12 does not sensitize cells to hypoxia. However, in contrast to survival experiments in vitro, hypoxic cells in the TME are not only oxygen deprived, but also exposed to nutrient starvation, growth factor deprivation and cycling hypoxia; combinations of stressors not examined in this study, but which could be lethal to ATG12 deficient cells. Previous studies show that inhibition of autophagy (ATG7, LC3B, CQ, 3-MA) does not change the cell intrinsic resistance to radiotherapy (47) However, recently, it was shown that inhibition of ATG12 sensitizes colorectal cancer cells to radiotherapy what can contribute to the improved therapy efficacy in our study (48). 
As a tumour suppressor, autophagy prevents cells from oncogenic transformation by suppressing accumulation of cyto- and genotoxic components. In various murine models it is shown that defective autophagy can lead to oncogenic transformation leading to lung and liver carcinomas or at least, contribute to the formation of pre-malignant lesions $(49,50)$. The high frequency of ATG12 deletion in head and neck cancers stimulated us to investigate if the loss of ATG12 contributed to malignant transformation and tumour development in the head and neck region. To prove that the loss of ATG12 plays a causal role in tumour development, we engineered conditional head and neck-specific ATG12\% mice based on a previous described model(51). In this model, we can specifically knock out ATG12 in the head and neck region and asses tumour formation based on the loss of ATG12 expression. In previous studies, SMAD4 ${ }^{-/}$was shown to lead to tumour formation in $74 \%$ of mice in 80 weeks and was therefore taken as a positive control (46). In our study, weekly inspection of the oral cavity did not show excessive tumour growth in experimental or control groups. However, dissection of the mice at the time of termination of the experiment (week 104) revealed lesions predominantly at the tongue, the thymus and salivary gland. Although these lesions were increased in ATG12\% compared to controls, pathological examination needs to confirm the observations. Although these findings need to be confirmed, they are in line with previous studies showing that defects in autophagy can contribute to the malignant transformation of normal cells to cancer cells.

The mortality rate in head and neck cancer is high due to uncontrolled loco-regional disease. A number of markers, such as tumour hypoxia and tumour cell proliferation, have been described to predict tumour control. It is well known that tumour hypoxia negatively affects treatment outcome. A meta-analysis has shown that the degree of tumour hypoxia is the most significant factor explaining variability in survival in head and neck cancers (52). Therefore there is a clinical need for endogenous hypoxia markers. So far, several endogenous markers such as HIF1 $\alpha$, CAIX, GLUTland others, have been identified to determine the hypoxic status of the tumour. However, expression of endogenous markers does not always reflect the tempero-spatial fluctuations of hypoxia due to its heterogeneous and dynamic characteristics. In contrast to endogenous markers of hypoxia, which are dependent on tumour hypoxia, ATG12 expression seems to predict if a tumour is hypoxic, and therefore has to potential to serve as a predictive marker for local and loco/regional control in HNSCC.

\section{Acknowledgments}

The authors would like to thank Hans Peters and Kathleen Daenen for their excellent technical support. This work was financially supported by the Dutch Cancer Society (KWF Grants UM 2012-5506 and 2015-7735 to K.R.), worldwide cancer research award 16-0265 (to K.R.) 


\section{References}

1. Rabinowitz JD, White E. Autophagy and metabolism. Science 2010;330:1344-8

2. Schaaf MB, Keulers TG, Vooijs MA, Rouschop KM. LC3/GABARAP family proteins: autophagy-(un)related functions. Faseb J 2016;30:3961-78

3. Klionsky DJ, Abeliovich H, Agostinis P, Agrawal DK, Aliev G, Askew DS, et al. Guidelines for the use and interpretation of assays for monitoring autophagy in higher eukaryotes. Autophagy 2008;4:151-75

4. Ichimura Y, Kirisako T, Takao T, Satomi Y, Shimonishi Y, Ishihara N, et al. A ubiquitin-like system mediates protein lipidation. Nature 2000;408:488-92

5. Tanida I, Ueno T, Kominami E. LC3 conjugation system in mammalian autophagy. Int J Biochem Cell Biol 2004;36:2503-18

6. Kuma A, Hatano M, Matsui M, Yamamoto A, Nakaya H, Yoshimori T, et al. The role of autophagy during the early neonatal starvation period. Nature 2004;432:1032-6

7. Rubinstein AD, Eisenstein M, Ber Y, Bialik S, Kimchi A. The autophagy protein atg12 associates with antiapoptotic bcl-2 family members to promote mitochondrial apoptosis. Mol Cell 2011;44:698-709

8. Saitoh T, Fujita N, Jang MH, Uematsu S, Yang BG, Satoh T, et al. Loss of the autophagy protein Atg16L1 enhances endotoxin-induced IL-1beta production. Nature 2008;456:264-8

9. Radoshevich L, Murrow L, Chen N, Fernandez E, Roy S, Fung C, et al. ATG12 conjugation to ATG3 regulates mitochondrial homeostasis and cell death. Cell 2010;142:590-600

10. Murrow L, Malhotra R, Debnath J. ATG12-ATG3 interacts with Alix to promote basal autophagic flux and late endosome function. Nature cell biology 2015;17:300-10

11. Karantza-Wadsworth V, Patel S, Kravchuk O, Chen G, Mathew R, Jin S, et al. Autophagy mitigates metabolic stress and genome damage in mammary tumorigenesis. Genes Dev 2007;21:1621-35

12. Mathew R, Kongara S, Beaudoin B, Karp CM, Bray K, Degenhardt K, et al. Autophagy suppresses tumor progression by limiting chromosomal instability. Genes Dev 2007;21:1367-81

13. Rouschop KM, Ramaekers CH, Schaaf MB, Keulers TG, Savelkouls KG, Lambin P, et al. Autophagy is required during cycling hypoxia to lower production of reactive oxygen species. Radiother Oncol 2009;92:411-6

14. Levine B, Kroemer G. Autophagy in the pathogenesis of disease. Cell 2008;132:27-42

15. Cliby W, Ritland S, Hartmann L, Dodson M, Halling KC, Keeney G, et al. Human epithelial ovarian cancer allelotype. Cancer Res 1993;53:2393-8

16. Eccles DM, Russell SE, Haites NE, Atkinson R, Bell DW, Gruber L, et al. Early loss of heterozygosity on 17q in ovarian cancer. The Abe Ovarian Cancer Genetics Group. Oncogene 1992; 7:2069-72

17. Russell SE, Hickey GI, Lowry WS, White P, Atkinson RJ. Allele loss from chromosome 17 in ovarian cancer. Oncogene 1990;5:1581-3

18. Tangir J, Muto MG, Berkowitz RS, Welch WR, Bell DA, Mok SC. A 400 kb novel deletion unit centromeric to the BRCA1 gene in sporadic epithelial ovarian cancer. Oncogene 1996;12:735-40

19. Qu X, Yu J, Bhagat G, Furuya N, Hibshoosh H, Troxel A, et al. Promotion of tumorigenesis by heterozygous disruption of the beclin 1 autophagy gene. J Clin Invest 2003;112:1809-20

20. Yue Z, Jin S, Yang C, Levine AJ, Heintz N. Beclin 1, an autophagy gene essential for early embryonic development, is a haploinsufficient tumor suppressor. Proc Natl Acad Sci U S A 2003;100:15077-82

21. Takamura A, Komatsu M, Hara T, Sakamoto A, Kishi C, Waguri S, et al. Autophagy-deficient mice develop multiple liver tumors. Genes Dev 2011;25:795-800

22. Wouters BG, van den Beucken $T$, Magagnin MG, Lambin P, Koumenis C. Targeting hypoxia tolerance in cancer. Drug Resist Updat 2004;7:25-40

23. Hockel M, Schlenger K, Aral B, Mitze M, Schaffer U, Vaupel P. Association between tumor hypoxia and malignant progression in advanced cancer of the uterine cervix. Cancer Res 1996;56:4509-15

24. Nordsmark M, Bentzen SM, Rudat V, Brizel D, Lartigau E, Stadler P, et al. Prognostic value of tumor oxygenation in 397 head and neck tumors after primary radiation therapy. An international multi-center study. Radiother Oncol 2005;77:18-24 
25. Brizel DM, Scully SP, Harrelson JM, Layfield LJ, Bean JM, Prosnitz LR, et al. Tumor oxygenation predicts for the likelihood of distant metastases in human soft tissue sarcoma. Cancer Res 1996;56:941-3

26. Gort EH, Groot AJ, van der Wall E, van Diest PJ, Vooijs MA. Hypoxic regulation of metastasis via hypoxiainducible factors. Curr Mol Med 2008;8:60-7

27. Gort EH, van Haaften G, Verlaan I, Groot AJ, Plasterk RH, Shvarts A, et al. The TWIST1 oncogene is a direct target of hypoxia-inducible factor-2alpha. Oncogene 2008;27:1501-10

28. Theys J, Jutten B, Habets R, Paesmans K, Groot AJ, Lambin P, et al. E-Cadherin loss associated with EMT promotes radioresistance in human tumor cells. Radiother Oncol 2011;99:392-7

29. Yang MH, Wu KJ. TWIST activation by hypoxia inducible factor-1 (HIF-1): implications in metastasis and development. Cell Cycle 2008;7:2090-6

30. Overgaard J. Hypoxic modification of radiotherapy in squamous cell carcinoma of the head and neck - A systematic review and meta-analysis. Radiother Oncol 2011

31. Rouschop KM, van den Beucken T, Dubois L, Niessen H, Bussink J, Savelkouls K, et al. The unfolded protein response protects human tumor cells during hypoxia through regulation of the autophagy genes MAP1LC3B and ATG5. J Clin Invest 2010;120:127-41

32. Minn H, Clavo AC, Grenman R, Wahl RL. In vitro comparison of cell proliferation kinetics and uptake of tritiated fluorodeoxyglucose and L-methionine in squamous-cell carcinoma of the head and neck. J Nucl Med 1995;36:252-8

33. Jozefczuk J, Drews K, Adjaye J. Preparation of mouse embryonic fibroblast cells suitable for culturing human embryonic and induced pluripotent stem cells. J Vis Exp 2012

34. Rouschop KM, Dubois LJ, Keulers TG, van den Beucken T, Lambin P, Bussink J, et al. PERK/elF2alpha signaling protects therapy resistant hypoxic cells through induction of glutathione synthesis and protection against ROS. Proc Natl Acad Sci U S A 2013;110:4622-7

35. Pramana J, Van den Brekel MW, van Velthuysen ML, Wessels LF, Nuyten DS, Hofland I, et al. Gene expression profiling to predict outcome after chemoradiation in head and neck cancer. Int J Radiat Oncol Biol Phys 2007;69:1544-52

36. Kaanders JH, Pop LA, Marres HA, Liefers J, van den Hoogen FJ, van Daal WA, et al. Accelerated radiotherapy with carbogen and nicotinamide (ARCON) for laryngeal cancer. Radiother Oncol 1998;48:115-22

37. Zhou Z, Wang D, Wang XJ, Roop DR. In utero activation of K5.CrePR1 induces gene deletion. Genesis 2002;32:191-2

38. Janssen HL, Ljungkvist AS, Rijken PF, Sprong D, Bussink J, van der Kogel AJ, et al. Thymidine analogues to assess microperfusion in human tumors. Int J Radiat Oncol Biol Phys 2005;62:1169-75

39. Horsman MR, Overgaard J. The impact of hypoxia and its modification of the outcome of radiotherapy. J Radiat Res 2016;57 Suppl 1:i90-i8

40. Jutten B, Keulers TG, Peeters HJM, Schaaf MBE, Savelkouls KGM, Compter I, et al. EGFRvIII expression triggers a metabolic dependency and therapeutic vulnerability sensitive to autophagy inhibition. Autophagy 2018;14:283-95

41. Patmore HS, Ashman JN, Stafford ND, Berrieman HK, MacDonald A, Greenman J, et al. Genetic analysis of head and neck squamous cell carcinoma using comparative genomic hybridisation identifies specific aberrations associated with laryngeal origin. Cancer Lett 2007;258:55-62

42. Schaaf MB, Cojocari D, Keulers TG, Jutten B, Starmans MH, de Jong MC, et al. The autophagy associated gene, ULK1, promotes tolerance to chronic and acute hypoxia. Radiother Oncol 2013;108:529-34

43. Jutten B, Keulers TG, Peeters HJM, Schaaf MBE, Savelkouls KGM, Compter I, et al. EGFRvIII expression triggers a metabolic dependency and therapeutic vulnerability sensitive to autophagy inhibition. Autophagy 2018:1-13

44. Rubinstein $A D$, Eisenstein M, Ber $Y$, Bialik S, Kimchi A. The autophagy protein Atg12 associates with antiapoptotic Bcl-2 family members to promote mitochondrial apoptosis. Mol Cell 2011;44:698-709

45. Lu SL, Reh D, Li AG, Woods J, Corless CL, Kulesz-Martin M, et al. Overexpression of transforming growth factor beta1 in head and neck epithelia results in inflammation, angiogenesis, and epithelial hyperproliferation. Cancer Res 2004;64:4405-10 
46. Bornstein S, White R, Malkoski S, Oka M, Han G, Cleaver T, et al. Smad4 loss in mice causes spontaneous head and neck cancer with increased genomic instability and inflammation. J Clin Invest 2009;119:340819

47. Schaaf MB, Jutten B, Keulers TG, Savelkouls KG, Peeters HJ, van den Beucken T, et al. Canonical autophagy does not contribute to cellular radioresistance. Radiother Oncol 2015;114:406-12

48. Hu JL, He GY, Lan XL, Zeng ZC, Guan J, Ding Y, et al. Inhibition of ATG12-mediated autophagy by miR-214 enhances radiosensitivity in colorectal cancer. Oncogenesis 2018;7:16

49. Qu X, Yu J, Bhagat G, Furuya N, Hibshoosh H, Troxel A, et al. Promotion of tumorigenesis by heterozygous disruption of the beclin 1 autophagy gene. J Clin Invest 2003;112:1809-20

50. Mortensen M, Soilleux EJ, Djordjevic G, Tripp R, Lutteropp M, Sadighi-Akha E, et al. The autophagy protein Atg7 is essential for hematopoietic stem cell maintenance. J Exp Med 2011;208:455-67

51. Lu SL, Herrington H, Reh D, Weber S, Bornstein S, Wang D, et al. Loss of transforming growth factor-beta type II receptor promotes metastatic head-and-neck squamous cell carcinoma. Genes Dev 2006;20:1331-42

52. Nordsmark M, Bentzen SM, Rudat V, Brizel D, Lartigau E, Stadler P, et al. Prognostic value of tumor oxygenation in 397 head and neck tumors after primary radiation therapy. An international multi-center study. Radiother Oncol 2005;77:18-24 


\section{Supplemental figure}

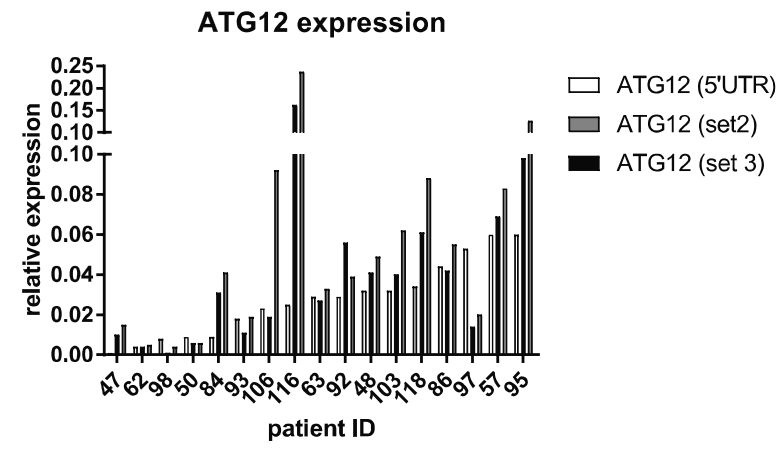

\section{Supplementary figure 1}

The identical pattern of the 3 independent primer pairs, indicate loss of the full ATG12 transcript expression 


\section{Chapter}

\section{Autophagy-dependent secretion: contribution to tumour progression}

Tom G.H. Keulers ${ }^{1 *}$, Marco B.E. Schaaf ${ }^{2 *}$, Kasper M.A. Rouschop ${ }^{1}$

${ }^{1}$ Maastricht Radiation Oncology (MaastRO) lab, GROW - School for Oncology and Developmental Biology, Maastricht University, Maastricht, the Netherlands

${ }^{2}$ Cell Death Research and Therapy (CDRT) Laboratory, Department Cellular and Molecular Medicine, KU Leuven, University of Leuven, Leuven, Belgium *authors contributed equally

Published in Frontiers in Oncology, 2016 Nov 25;6:251. 


\section{Abstract}

Autophagy is best known as a lysosomal degradation and recycling pathway to maintain cellular homeostasis. During autophagy, cytoplasmic content is recognized and packed in autophagic vacuoles, or autophagosomes, and targeted for degradation. However, during the last years, it has become evident that the role of autophagy is not restricted to degradation alone but also mediates unconventional forms of secretion. Furthermore, cells with defects in autophagy apparently are able to reroute their cargo, like mitochondria, to the extracellular environment; effects that contribute to an array of pathologies. In this review we discuss the current knowledge of the physiological roles of autophagy-dependent secretion, i.e. the effect on inflammation and insulin/ hormone secretion. Finally, we focus on the effects of autophagy-dependent secretion on the tumour microenvironment and tumour progression. The autophagy mediated secreted factors may stimulate cellular proliferation via auto- and paracrine signaling. The autophagy mediated release of immune modulating proteins change the immunosuppressive tumour microenvironment and may promote an invasive phenotype. These effects may be either direct or indirect through facilitating formation of the mobilized vesicle, aid in anterograde trafficking or alterations in homeostasis and/or autonomous cell signaling. 


\section{Autophagy}

Autophagy is a catabolic process required to maintain cellular homeostasis by lysosomal degradation of aged/damaged organelles (e.g. mitochondria), protein aggregates and pathogens. Autophagy commences with the formation of an initial cup-shaped membrane (phagophore) that elongates while (non-) selectively capturing cytoplasmic constituents into a double-membrane vesicle (autophagosome). Ultimately, the autophagosome fuses with a hydrolase and protease containing lysosome for degradation of the content. The end-products are recycled into the cytosol and are reused in processes including protein synthesis and ATP production.

During the last decade, extensive research revealed that at least 38 Autophagy related proteins (ATGs) comprise the core autophagy machinery that mediate initiation, elongation, cargo recruitment and fusion with lysosomes (1). Furthermore, the yeast atg8 orthologues of the LC3/GABARAP protein family fulfil specialized roles in the execution of autophagy (2). This family consists of 7 active members (LC3A (two splicing variants; LC3A-a and LC3A-b), LC3B, LC3C, GABARAP, GABARAPL1, and GABARAPL2) which share a high mutual homology, including a conserved C-terminal glycine residue for phosphatidylethanolamine (PE) conjugation to allow membrane association. Conjugation of the LC3/GABARAP protein family members to $P E$ is required for expansion and closure of the phagophore. This process is controlled by 2 ubiquitin like conjugation systems, a process closely orchestrated the ATG4, -7, and -3 and the ATG12-ATG5ATG16L1 complex (9).

Despite the high grade of homology, the protein family members harbour individual roles in autophagy but are associated with autophagy unrelated functions as well like receptor trafficking and (2).

Autophagy (and related defects) is associated with several pathologies, including neuropathologies, Crohns disease and cancer. In cancer, autophagy is considered a double edged sword; i.e. in healthy tissue, autophagy plays a cytoprotective role by maintaining homeostasis through degradation of cytotoxic constituents, which otherwise may trigger tumorigenic events. Nevertheless, once a tumour is formed, autophagy contributes to survival of cancer cells in areas deprived of nutrients of oxygen (hypoxia) $(3,4)$, a common feature of solid tumours that contributes to tumour progression, therapy resistance and metastases formation(5).

Yet, accumulating research shows that the homeostatic role of autophagy and its related proteins is more elaborate than the degradation of cytoplasmic content alone. Autophagy not only contributes to intracellular homeostasis, but also seems to contribute to tissue homeostasis by mediating intercellular communication. Peptides, proteins and hormones that fail to enter the conventional secretory system due to the lack of a leader/secretion signal sequence can be secreted in an autophagy-dependent manner. 
In this review we list the current knowledge on the role of the autophagy machinery in autophagy-dependent secretion and specifically focus on factors that may influence tumour progression.

\section{(Un) conventional protein secretion}

In eukaryotes, a classical route for proteins to be secreted is the endoplasmic reticulum (ER)-to-Golgi route. Herein, proteins are directed towards the ER by their amino terminal signal peptide (leader peptide) and progress through vesicular flow to the Golgi. In brief, after ER translocation, proteins are oligomerized and packed into carrier vesicles that exit the ER at specialized regions. These vesicles assemble into vesiculo-tubular structure intermediates known as the ER-to-Golgi intermediate compartments (ERGIC) that, by lateral communication sorts proteins for further anterograde flow to the Golgi complex. In the Golgi, proteins are glycosylated to ensure proper protein structure, increase stability and to allow interactions with target proteins (6). In the trans-Golgi network, secretory proteins are sorted into secretory vesicles that deliver their content to the plasma membrane to result in secretion (7). An increasing number of secreted proteins that lack the leader peptide have been identified. These proteins require alternative pathways to be secreted in a regulated fashion. This implies differences in vesicle formation, sorting and transportation. Multiple alternate, non-classical pathways exist and are commonly referred to as unconventional protein secretion and include both non-vesicular and vesicular mechanisms (8). For example, cytosol-residing proteins, including $A B C$ transporter mediated yeast a-factor (9) or mammalian fibroblast growth factor 2 (FGF-2) are directly transported across the plasma membrane. For FGF2 secretion this is presumed to be mediated through $\mathrm{PI}(4,5) \mathrm{P}_{2}$-induced oligomerization followed by membrane insertion and translocation (10). Other proteins are sorted into vesicular intermediates that fuse with the plasma membrane to release their content into the extracellular space ((interleukin $1 \beta(l-1 \beta)$ and $\mid$ 18 (11)), in part dependent on proteins required for autophagy execution $(12,13)$. This suggests that autophagyproteins are required to produce secretory vesicles or that autophagosomes gain exocytic features. These processes in autophagy-mediated secretion are distinct from its degradative compartment (as reviewed in more detail in (14)). In this context, the trafficking, tethering, docking and plasma membrane fusion events would differ from canonical autophagy and resemble exocytic mechanisms. Herein, secretory vesicles are diverted from the endocytic system to be released from multivesicular bodies (MVB). These secretory vesicles are then transported to the plasma membrane for content release. For example, the interaction between autophagosomes and the endocytic/MVB pathway (reviewed in (15)) are required for acetyl-CoA-binding protein(Acb1)-secretion and requires the fusion of Acb1-containing autophagosomes with endosomes or MVBs before plasma membrane fusion (16). 
The involvement of autophagy-related proteins, in protein secretion indicates that cells utilize these effectors in a surprising mechanism of unconventional secretion. Here, we will discuss the data that support a relation between autophagy and autophagy-related proteins with secretory pathways (table 1). First, we will elaborate on the physiological roles of autophagy in secretory events. Second, cancer cells utilize secretory mechanisms to communicate with stroma and surrounding cancer cells, thereby inducing alterations that influence tumour progression including immunoevasion, immunosuppression, auto- and paracrine signalling and angiogenesis.

Table 1: Effects on the tumour microenvironment

\begin{tabular}{|c|c|c|c|}
\hline Protein(s) & mechanism & Effect & ref \\
\hline \multicolumn{4}{|c|}{ 1. Influencing immunogenic cell death to evade immune surveillance } \\
\hline ATP & $\begin{array}{l}\text { ATG5 } \\
\text { knockdown }\end{array}$ & $\begin{array}{l}\text { After radiotherapy and MTX exposure "eat me signal" for } \\
\text { immune cells. stimulus for DC recruitment, IFNy-producing CD4 } \\
\text { and CD8 T cells }\end{array}$ & $\begin{array}{l}(65)(63) \\
(64)\end{array}$ \\
\hline HMGB1 & ATG $5^{f l / f l}$ & $\begin{array}{l}\text { Promote processing and presentation of tumour antigens by DCs, } \\
\text { enhanced immuno surveillance. }\end{array}$ & $5,(11)$ \\
\hline \multicolumn{4}{|c|}{ II. Cytokine release and influence on the tumour microenvironment } \\
\hline IL1-B, IL-6, IL2 & $\begin{array}{l}\text { GABARAP } \\
\text { Knockout mice }\end{array}$ & Increased secretion by macrophages & $(81)$ \\
\hline IFN $\gamma$ & $\begin{array}{l}\text { GABARAP } \\
\text { Knockout mice }\end{array}$ & Increased secretion by lymphocytes & $(81)$ \\
\hline $\begin{array}{l}\text { CXCL9, CXCL10 } \\
\text { and CXCL11 }\end{array}$ & $\begin{array}{l}\text { FIP200 } \\
\text { conditional } \\
\text { knockout }\end{array}$ & Enhanced secretion, leading to improved immuno surveillance & (49) \\
\hline \multicolumn{4}{|c|}{ III.a. Pro-metastatic: Driving an invasive behavior of cancer cells } \\
\hline \multirow[t]{2}{*}{$\begin{array}{l}\text { LIF, FAMC3, } \\
\text { DKK3, IL-8 }\end{array}$} & $\begin{array}{l}\text { ATG7 } \\
\text { knockdown }\end{array}$ & $\begin{array}{l}\text { These factor promote metastasis via MMP2 upregulation (IL-8), } \\
\text { epithelial to mesenchymal transition (FAM3C, DKK3, LIF) and } \\
\text { promotion of angiogenesis (IL-8 and DKK3) }\end{array}$ & $(83)$ \\
\hline & $\begin{array}{l}\text { ATG7, } \\
\text { ATG12,ATG3 } \\
\text { knockdown } \\
\text { chloroquine or } \\
\text { bafilomycin A1 }\end{array}$ & \multicolumn{2}{|l|}{$\begin{array}{l}\text { Autophagy deficient HRAS }{ }^{112} \text { transformed breast cancer cell lines (82) } \\
\text { display reduced invasive protrusions. Conditioned medium of } \\
\text { autophagy-proficient cells rescued the invasive phenotype }\end{array}$} \\
\hline $\begin{array}{l}\text { IL6, CCL-2, CCL- } \\
\text { 20, VEGFA, } \\
\text { MMP2 }\end{array}$ & $\begin{array}{l}\text { 3-MA, ATG5 } \\
\text { and ATG7 } \\
\text { knockdown }\end{array}$ & $\begin{array}{l}\text { TLR3 and TLR4 activation leads to autophagy - dependent } \\
\text { secretion of these factors associated with a migratory and } \\
\text { invasive phenotype of lung cancer cells. }\end{array}$ & $(85)$ \\
\hline IL6 & $\begin{array}{l}\text { ATG7 or beclin } \\
\text { knockdown }\end{array}$ & $\begin{array}{l}\text { Autophagy deficiency lead to an in- or decrease in low or high } \\
\text { autophagic breast cancer cells respectively. Autophagy- } \\
\text { dependent secretion of IL- } 6 \text { are able to promote mammosphere } \\
\text { formation and may be important in CSC maintenance }\end{array}$ & (87) (86) \\
\hline
\end{tabular}

III.b. Pro-metastatic: Proper Weibel-Palade body formation in vascular endothelium to facilitate metastasis

WPB proteins

IV. Chemoresistantance

HMGB1 Autophagy is important to sustain secretion of WPBs containing
proteins that influence tumour progression

HMGB1 causes doxorubicin resistance in neighboring breast 


\section{Physiological roles of autophagy mediated secretion}

The first evidence for the involvement of autophagy-related proteins in protein secretion was obtained in yeast. Acyl coenzyme A-binding protein (Acbp) is secreted through an unconventional secretory pathway that depends on components of the autophagic process (Atg5, Atg7, Atg8, Atg12) (16). Importantly, this study also demonstrated that autophagy connects with compartments (multivesicular bodies) of endosomal trafficking and SNARE-dependent membrane fusion events are both required for the Acbp secretion. Since the discovery of autophagy-dependent secretion in yeast, also several mammalian factors have been identified to be dependent on autophagy-related proteins for their secretion.

Phosphoinositides are membrane lipids with specific recognition domains for the recruitment of cytosolic proteins and are involved in the regulation of membrane transport. So far, seven phosphoinositides have been described. They can be converted into each other by phosphoinositide kinases and phosphatases (17). PIKfyve is one of those converting kinases and has been implicated in membrane transport events. The substrate of PIKfyve, PI(3)P, plays a critical role in the initiation of autophagy and autophagosome maturation (18). Inhibition or depletion of PIKfyve by apilimod or siRNA resulted in decreased autophagic flux, probably caused by an impaired autophagosome/lysosome fusion. Interestingly, prostate cancer cells incubated with the PIKfyve inhibitor apilimod, secrete more autophagy associated proteins (NBR1, P62, OPTN and CACO2 and membrane-bound $\mathrm{LC} 3 \mathrm{~B}$ ), however proteins involved in earlier stages of autophagy were not observed (ATG2A, ATG5, ATG16L). Analysis of density gradients reveal that these autophagy associated proteins are expressed on vesicles and appear as autophagosome subpopulations, suggesting induced secretory autophagy (19).

\section{Cytokines and inflammation}

Autophagy is able to suppress inflammasome activation through maintaining mitochondrial homeostasis (20). In line, Zhang et al observed increased inflammasome activation in macrophages after sepsis in Gamma $(\gamma)$-aminobutyric acid type A receptor (GABAAR)associated protein (GABARAP) knockout mice (20). Secretion of cytokines is central in inflammasome activity. Well-documented cytokines that are associated with unconventional secretion in an autophagy-dependent manner are interleukin-1 beta (IL-1B) and IL-18. Dupont et al demonstrated in bone-marrow derived macrophages during conditions of induced autophagy (starvation or Torkinib treatment) that secretion of IL-1B increased, which was reduced in the absence of ATG5. Consistent with data obtained from yeast Acbp, similar additional factors were required (as mammalian equivalents of yeast Golgi-associated protein (GRASP55) and small GTPase Rab8a), indicating a conserved system or cooperation (11). 
Interestingly, three dimensional STochastic Optical Reconstruction Microscopy (STORM) demonstrated that IL-1B was not only present in the inner vesicle of the autophagosome, but was already recruited to the emerging phagophore. In this, IL-1B is actively translocated across the membrane and accumulated in the intermembrane space of the mature autophagosome (21). This, in contrast to the sequestration of degradable autophagosomal content which is located in the inner vesicle as a result of cytoplasmic engulfment and closure of the phagophore, this novel mechanism indicates differences in cargo recruitment and indicates formation of different vesicles by the autophagy-related proteins that may guide the distinct fate of the produced vesicle.

\section{Role of autophagy-dependent secretion in establishing a barrier for infection}

Paneth cells are specialized epithelial cells present at the base of the crypts of Lieberkuhn. These cells control the intestinal microbiota through secretion of intracellular granules that contain antimicrobial peptides and lysozyme. Autophagy-proteins are essential for Paneth cell function. Knockdown of crucial autophagy genes (ATG16L1, ATG5 or ATG7 $(22,23)$ ) results in alterations in Paneth cell granules, as illustrated by fewer and aberrant granules with diffuse lysozyme patterns and decreased presence of Iysozyme in mucus. Due to its relevance in establishing a microbial barrier, the ATG16L1 gene was annotated as a Crohns disease (CD) risk allele (24) and the phenotype is similar to the abnormalities found in Paneth cells of CD patients.

In contrast to CD where the ileum and colon are often affected, ulcerative colitis is restricted to the colon only. Conditional knockdown of ATG7 in the colon (GICNAc6ST-2$\mathrm{Cre}$ ) increased bacterial colonization in crypts. This phenotype was associated with a decreased release of mucin from goblet cells and reduced expression of antimicrobial and antiparasitic peptides (angiogenin 4 , interlectin 1 and relm $\beta$ ). Hence, colonic ATG7 knockdown results in increased susceptibility to the development of UC-like colitis (25).

In line with autophagy-dependent secretion by intestinal tissue, ATG5 and ATG16L1 deficiency results in reduced secretion of mucins (MUC5AC) by human tracheal epithelial cells (26).

Together, autophagy-dependent secretion is required to maintain effective antibacterial barriers by epithelial cells. In line, ATG16L1 conditional knockout mice (VillinCre) are more susceptible to Salmonella typhimurium infection (27). Although, beyond the contribution of establishing a barrier function, autophagy is required for limiting bacterial proliferation through ATG16L1 and LC3C dependent degradation of cytosolresiding Salmonella (xenophagy) (28). Evidently, autophagy is a prerequisite for different cell types to maintain their function (e.g. mucus secretion) thereby and protection against inflammatory disorders. 


\section{Insulin secretion}

Pancreatic $\beta$-cells are specialized in secretion of insulin in response to high levels of blood glucose concentrations. Release of insulin is mediated by the fusion of insulincontaining vesicles with the plasma membrane. These insulin-containing vesicles are transported towards the plasma membrane. Gamma $(\gamma)$-aminobutyric acid type A receptor (GABAAR)-associated protein (GABARAP), one of seven mammalian variants of yeast Atg8, which is already known to be implicated in multiple cellular functions including fusion events in autophagy (29) and receptor trafficking (30) has recently been demonstrated to mediate insulin secretion. Herein, GABARAP binds insulin-containing vesicles in a PE conjugation-dependent mechanism (31)). Together with its microtubules-binding feature (residue 10-22 (30)), GABARAP allows vesicle presentation to the motor protein KIF5B (located at microtubules) and facilitates vesicle mobility and insulin release (32).

\section{NPY secretion}

In rat adrenal medullar pheochromocytoma derived cells, the neurites display ATG16L at dense-core vesicles. Although these vesicles do not co-localize with LC3, release of peptide hormone neuropeptide $Y$ (NPY) is dependent on ATG16L1 functioning as a Rab33a (which regulates membrane trafficking events) effector. Interestingly, NPY secretion was not altered in cells that were deficient in autophagy by overexpressing ATG4B ${ }^{\text {C74A }}$ or knockdown of ATG13 or ULK1 and indicates a highly specialized role for ATG16L1 in hormone secretion that is independent of other canonical autophagy associated proteins (33).

\section{Autophagy-mediated secretion of Weibel-Palade bodies in vascular endothelial cells}

Vascular injury stimulates endothelial cells to secrete factors to promote repair. Endothelial secretory granules (Weibel-Palade bodies (WPBs)) contain active molecules including von Willebrand factor (VWF), P-selectin, interleukin-8, angiopoietin-2 and endothelin-1. The intracellular WPBs are characterized by striations parallel to its longitudinal axis and are delineated by a membrane. When secreted, the multimeric hemostatic VWF is tethered to the connective tissue to mediate platelet adhesion at sites of vascular injury. The processing of VWF to a mature form is important in the WPB formation. Treatment with chloroquine or bafilomycin $\mathrm{A} 1$, that both raise lysosomal $\mathrm{pH}$, and knockdown of ATG7 or ATG5 reduce the number of endothelial WPBs due to incorrect processing of VWF. In line, endothelial specific deletion of Atg7 in mice led to reduced epinephrine-induced plasma vWF levels and increased bleeding time. Thus, autophagy in endothelial cells aids in hemostasis by proper maturation of WPBs (34). 


\section{Osteoclastic bone resorption}

Bone remodeling is a lifelong process of bone degradation and formation important for bone healing after injury, bone restructuring and sustaining bone homeostasis. Osteoclasts cooperate with osteoblasts in bone remodeling in which osteoclasts are responsible for bone degradation and reabsorption of mineralized bone matrix. The osteoclasts are large multinucleated cells that are characterized by their ruffled border by which contact area with the bone is increased. Fusion of secretory lysosomes with the ruffled border causes release of matrix-degrading factors including cathepsin $\mathrm{K}$ which aids bone matrix degradation. ATG5 or ATG7 deficient osteoclasts lack a normal ruffled border, impaired localization of secretory factors including cathepsin $K$ and eventually have impaired bone resorption. Importantly, development of osteoclasts was not aberrant indicating that the autophagy deficiency led to functional impairment independent of sustaining cellular homeostasis. Moreover, secretory lysosome formation was unaffected, whereas Rab7 (key factor for lysosome fusion events) localization to ruffled border was ATG5 dependent. Together, these data indicated that autophagy-related proteins aid in secretory events at the osteoclast ruffled border by directing fusion of the secretory lysosome with the plasma membrane (35).

In conclusion, autophagy is important for secretory functions of various cell types. Important to note is that autophagy can have either direct or indirect contribution to protein secretion. For example, autophagy related proteins directly facilitate protein secretion by mediating cargo sequestration (as IL-1B) or vesicle trafficking (as insulin), but autophagy also maintains cellular homeostasis that is important to preserve the specialized function (IL-1B and VWF) and primes the plasma membrane for proper release of autophagy-independent secretory vesicle (as in osteoclasts). Furthermore, for sustaining a microbial barrier, autophagy's contribution is dual as both a canonical form of autophagy (xenophagy) and the regulation of important secretory factors as mucin contribute to prevent pathogen invasion.

\section{Secretory autophagy: waste disposal?}

Damaged and aggregated proteins and aged organelles are typically degraded by autophagy. Substrates for autophagy are ubiquitylated and recognized by autophagy receptors and degraded. Recent work indicates that defective or saturated autophagy, i.e. by defective autophagosome/ lysosome fusion results in cargo secretion into the extracellular environment.

Lysosomal dysfunction is associated with the secretion of aggregation prone proteins that are associated with neurodegenerative diseases as Parkinson's and Alzheimer's disease. $\alpha$-Synuclein is a presynaptic neuronal protein that is genetically and neuropathologically linked to Parkinson's disease. Wild type $\alpha$-synuclein is typically 
degraded by the autophagy and the proteasome (36). Interestingly, tubulin polymerization-promoting protein/p2 5 a, expressed in the CNS, sorts-synuclein into autophagsomes but simultaneously prevents its degradation through inhibition of autophagosome/lysosome fusion. Instead p2 5 a controls-ynuclein clearance by its release in the extracellular environment in an autophagy-dependent manner (37).

Alzheimers' disease is characterized by the accumulation of intracellular Amyloid beta $(A B)$ peptide and tau aggregates and extracellular $A B$ plaques. In normal conditions, intracellular proteins are cleared by autophagy and autophagosomes are resolved in the process. However, during Alzheimer disease, autophagosomes accumulate, indicative of impaired autophagy. Autophagy-deficiency (ATG7 knockout) in excitatory neurons results in intracellular $A B$ accumulation, confirming its role in clearance of $A B$ aggregates by autophagy. Although $A B$ increased intracellularly, extracellular $A B$ plaque formation was drastically reduced. Reconstitution of ATG7 expression by lentiviral transduction, rescued the secretory phenotype. In parallel, pharmacologic modulation by either induction or inhibition of autophagy, by rapamycin or spautin-1, increased and reduced extracellular $A B$ release respectively. Thus, autophagy influences intracellular transport and secretion of $A B(38)$.

Mitochondria, the energy producing centres of the cell, generate ROS as a byproduct of oxidative phosphorylation. In many cancers, ROS production is increased due to mutations in mitochondrial DNA, hypoxia and a disturbed metabolism, leading to cancer progression(39). The homeostasis of mitochondrial ROS plays an important role in the regulation of autophagy. Depolarized and ROS leaking mitochondria are typically degraded by a selective form of autophagy, mitophagy. In depolarized mitochondria, PINK recruits Parkin to mediate selective removal of the organelle in a degrative autophagy-dependent manner. However, a recent report shows that there is an alternative way to maintain mitochondrial homeostasis in the cell. Mesenchymal stem cells (MSC) pack depolarized mitochondria in microvesicles and release them in the extracellular environment to outsource mitophagy where they are recognized by and transferred to macrophages. These released microvesicles are highly enriched in LC3 and ATG12 compared to whole-cell extracts. This mitochondrial transfer probably serves to increase MSC survival. (40). Similarly, LPS stimulated rat hepatocytes secrete mitochondrial proteins CPS1 and COXIV, a component of the mitochondrial respiratory chain and associated with the inner mitochondrial membrane, and mitophagy related proteins PARK2 and PINK1 and LC3B-II. These effects are inhibited by the autophagy inhibitor 3Metyladenine or after Atg5 knockout. Suggesting a role for autophagy in the secretion of mitochondria after LPS stimulation (41).

Also endothelial cells are able to release vesicles with autophagosome characteristics. During apoptosis, endothelial cells release in addition to apoptotic bodies, vesicles in an unconventional manner(42). Ultrastructural analysis by electron microscopy showed single membrane vesicles up to 10 um which contained structures of mitochondria, multivesicular bodies and autophagosomes. Further proteomic analysis re- 
vealed the release of autophagy associated proteins ATG16L1, LAMP2 and LC3B. The biological function is of this phenomenon remains to be elucidated (43).

The previous section lists the evidence that cells are able to release autophagic vesicles into the extracellular environment. Vesicle release during defects in the autophagic process, specifically during autophagosome/ lysosome fusion, suggests alternative mechanisms in waste removal.

\section{Effects on the tumour microenvironment}

Regardless of the clinical advances in the past decades that have improved cancer patient outcome, cancer is still one of the leading causes of death in the world. Importantly, the efficacy of treatment strategies is heavily influenced by cancer cell autonomous features but also by the tumour microenvironment. Solid tumours consist of a variety of cell types, including the cancer cells, endothelial cells, immune cells, fibroblasts and contain well- and poorly perfused areas that results in inefficient nutrient and oxygen supply $(44,45)$. Normal (non-transformed) cells in the tumour microenvironment (TME) are reprogrammed by the cancer cells to their benefit. This is exemplified by growth supporting angiogenesis and the suppression of anti tumour immunity. Importantly, an existing connection between autophagy and tumorigenesis has already been established. For example, deletion of a single BECN1 allele (Beclin1 protein important regulator of autophagy (46)) predisposes mice to spontaneous tumour development $(47,48)$. Further, depletion of FIP200 (important for autophagy initiation) in mammary cancer cells inhibits tumour initiation and progression including metastases (49). Autophagy in cancer cells supports their survival (by aiding the high energy demand) and abets resistance to metabolic and oxidative stresses (e.g. hypoxia) (50-55). Although this role is well established, the contribution of autophagy related intercellular communication that influences tumour progression through evasion of immunosurveillance, immunogenic cell death, angiogenesis and an invasive phenotype is an emerging field with great interest. For example, in cancer the RAS genes HRAS and KRAS are frequently mutated. Although the exact role of autophagy in tumour progression of RAS mutated tumours is still under debate, autophagy seems to be dispensable for the growth and survival of KRAS mutated cancer cell lines derived from human tumours (56). However when nonRAS mutated cells are transformed with oncogenic RAS, these cells are highly dependent on autophagy for tumorigenic events (57). In addition, the invasive phenotype of HRASV12-transformed breast cancer cells is reduced in ATG7 knockdown cells. This invasive phenotype could be rescued by incubating these autophagy deficient cells with conditioned medium of autophagy proficient cells. This supports an autophagydependent secretory system that supports tumour progression (58).

In the next section, the current knowledge on proteins secreted through autophagymediated processes that influence tumour progression is discussed (table 2). 


\section{Influencing immunogenic cell death to evade immunosurveillance}

Under normal circumstances, immune cells (including dendritic cells (DCs), natural killer (NK) cells and T cells) recognize and eliminate newly formed neoplastic cells due to their high immunogenic nature as a result of their mutational burden (immunosurveillance). Cancer cells that have obtained an immune evasive phenotype are able to circumvent recognition and subsequent elimination by the cooperative immune cells. These cancer cells are then selected for characteristics that circumvent local immunosurveillance and contribute to the growth of the lesion. With recent advances, the immune evasive feature is a topic of interest for the development of therapeutic strategies. Ideally, the elicited cancer therapy-induced cell death should provoke an immunogenic chain reaction that includes boosting the immune system to tilt the balance towards recognition rather than evasion, called immunogenic cell death (ICD). ICD invokes the release of immunomodulatory proteins (damage-associated molecular patterns (DAMPs) that incite anti tumour immunity (59). ICD can be induced by selective chemotherapeutics including mitoxantrone (MTX) and oxaliplatin (OXA) and radiotherapy (reviewed in (60)). Cancer cells undergoing ICD stimulate and activate the innate immune cells. Subsequently this can result in the cross-priming of the adaptive immune system for the antigens of dying cancer cells, thereby leading to an effective activation of anti tumour immunity. This can elicit a long-term therapeutic effect (even after therapy has stopped) and is fundamental to observed abscopal effects. In line, clinical studies have demonstrated that lymphopenia negatively affects chemotherapeutic response of solid tumours (61) and that ICDassociated DAMPs can be used as predictive and prognostic biomarkers (62).

Important immunogenic DAMPs that are displayed by cells undergoing ICD are secretion of ATP, surface exposure of calreticulin (CRT), release of heat shock proteins and high mobility group box 1 (HMGB1). Interestingly, ATG5 knockdown in colon cancer cells reduces ATP release after radiotherapy (63) and MTX exposure (64), which was associated with a decreased effect on tumour growth inhibition. Interestingly, in autophagy deficient cells no differences in CRT surface exposure or HMGB1 release were observed (64). The relevance of autophagy-dependent radiotherapy-induced ATP release was further supported by the observation that treatment with an inhibitor of ecto-ATPase only increased radiosensitivity in immunocompetent but not immunodeficient mice. Here, a partial rescue of lymphocyte infiltration indicates that the autophagy-dependent radiotherapy-induced ATP release enhances anti-tumour immunity (63). Similarly, for MTX treatment of osteosarcoma cells it was also shown that ATG5 is required for ATP secretion. Interestingly, on a more mechanistic level, Martins et al demonstrated that ATP (stored in lysosomes) is released upon MTX and OXA treatment and is associated with LAMP1 (lysosomal marker) translocation to the plasma membrane. Nonetheless, a role for autophagy seems to be maintaining an intracellular ATP pool (may even be cargo sequestration) required for (LAMP1+) lysosome-dependent ATP release. Accordingly, the replenishment of ATP to lysosomes was reduced when 
autophagy genes were knocked down (65). ATP release conveys an important "eat me" signal for immune cells. Once it is released, ATP may attract innate effector cells of the immune system into the tumour bed. Consistently, it was demonstrated that autophagy-dependent ATP release from MTX treated colon cancers was a stimulus for DC recruitment, IFNY-producing CD4 and CD8 T cells that had favourable effects on MTX sensitivity (64). Correspondingly, ATG7 in a genetically-induced melanoma mouse model was required for MTX-dependent growth inhibition that was reliant on functional CD4 and CD8 T cells (66). Further, caloric restriction or treatment with caloric restriction mimetics, that increase autophagy activity, enhance autophagy-dependent ATP release and improve MTX-induced tumour growth delay in a T cell dependent fashion (67).

Oppositely, a different study using a different ICD inducer (photo-oxidative ER stress inducer hypericin) has demonstrated autophagy-independent ATP release, but observed enhanced surface CRT exposure when autophagy was attenuated. The enhanced DC maturation and IL-6 secretion further promote IFNY producing T lymphocytes (68). Interestingly, surface CRT exposure after MTX or hypericin treatment could be ablated in cells lacking lysosome associated LAMP2A, an essential gene for a chaperonemediated autophagy (69). Moreover, regarding ATP it was demonstrated that extracellular residing ATP does not relay an immunogenic response per se (70) and further illustrates the context dependence of effects resulting in immunogenicity.

The involvement of autophagy-related proteins in the release of the DAMP HMGB1 has been demonstrated using ATG ${ }^{f l / f l} \mathrm{Cre}^{+}$bone marrow-derived macrophages (11) and dying glioma cancer cells (in which HMGB1 was found in a subset of autophagosomes before release (71). The released HMGB1 by dying cancer cells can bind Toll-like receptor (TLR-) 4 and promotes the processing and presentation of tumour antigens by DCs. This leads to cross-priming of T-cells and enhances immunosurveillance (72). Furthermore, endothelial cell exposure to HMGB1 triggers pro-angiogenic effects (73) including endothelial cell migration, sprouting and induction of an autocrine signalling cascade that results in elevated expression of leukocyte adhesion molecules ICAM-1, VCAM-1 and E-selectin. Moreover, HMGB1 induced expression of VEGF-A, VEGFR1, VEGFR2 and neuropilin-1 (74) and stimulation of angiogenesis (75).

In conclusion, ICD is an important pillar of therapy-induced anti-tumour immunity as it relays important signalling to the immune system, including DCs. DC stimulation may be important to induce tumour cure as demonstrated by ICD-based DC-vaccines in high grade glioma bearing mice (76). Nevertheless, the influence of autophagy on the display of DAMPs may be ICD-inducer dependent and requires further understanding for effective use.

\section{Cytokine release and influence on the tumour microenvironment}

ATP can bind the P2RX7 receptor and activate the NOD-like receptor family, pyrin domain containing 3 (NLRP3) inflammasome in DCs and macrophages (77). This inflam- 
masome activation can be suppressed by autophagy (20), which would be favorable as inflammasome activation and subsequent IL-1B release by e.g. macrophages have protumorigenic effects. However, the P2RX7 receptor impairs autophagy by blocking lysosomal function and stimulates release of vesicles with autophagolysosome characteristics (78). This suggests that activation of the P2RX7 receptor leads to a secretory phenotype, via inhibition of autophagy. Depletion of IL-1B arrests growth in melanoma (79) and macrophage-derived IL-1B induced IL-1 7 expression from $\gamma \delta T$ cells resulted in expansion of tumour associated neutrophils that suppress cytotoxic $T$ cells in breast cancer, resulting in increased number of pulmonary and lymph node metastases (80). Alternatively, in a murine colon carcinoma model autophagy-dependent ATP release after MTX treatment promoted recruitment of IFNy-producing CD8+ T cells into the tumour in an IL-1B-mediated fashion (64).

Lipopolysaccharide (LPS)/LPS+ATP stimulated GABARAP Knockout macrophages to secreted more IL-1B and IL-6. In addition, GABARAP knockout lymphocytes produced more IL-2 and interferon- $\gamma$. In this model, GABARAP KO was associated with reduced tumour incidence. These effects were validated on tumour control in a melanoma tumour cell inoculated model (81) and indicate that GABARAP in non-cancerous cells is sufficient to sustain pro-tumorigenic effects potentially due to control of cytokine secretion.

A study using MMTV-PYMT mouse model of breast cancer-bearing a conditional deletion of autophagy gene FIP200 shows that these tumour cells have a different chemokine secretion profile than FIP200 proficient cells. The TME polarizes towards an improved immunosurveillance as enhanced secretion of chemokines including CXCL9, CXCL10 and CXCL11 lead to increased infiltration of IFN $\gamma$-producing CD8+ and CD4+ T cells (49).

In conclusion, autophagy related secretion is important in controlling the cytokine profile of different cell types.

\section{III.a. Pro-metastatic: Driving an invasive behavior of cancer cells}

Oncogenic mutations in RAS are highly prevalent in cancers and drive different protumourigenic features including proliferation, survival and invasion. Autophagy deficient HRAS $^{\mathrm{V} 12}$ transformed breast cancer cell lines display reduced invasive protrusions in genetic knockdown models (including ATG7, ATG12 and ATG3) and after pharmacological inhibition (chloroquine or bafilomycin A1). Addition of conditioned medium of autophagy-proficient cells rescues the invasive phenotype, indicating a role for autophagydependent secretion in triggering cellular migration. Correspondingly, the pulmonary metastatic potential of HRAS ${ }^{\mathrm{V} 12}$ tumours is reduced in autophagy deficient cells, effects dependent on autophagy-related secretion (58). Despite these results in this model, the role of IL-6 in tumour progression is ambiguous in literature as both pro-tumorigenic 
(metastasis, angiogenesis, immune suppression) and anti-tumorigenic (CD8+ T cell trafficking to lymph nodes and tumours) effects on the TME are described (82).

Kraya et al observed a cytokine profile that differed between melanoma cells with high and low autophagy activation that could be mimicked by introducing ATG7 knockdown in an autophagy high cell line. The main secretory factors the authors focused on that were dependent on autophagy(-protein) are, leukemia inhibitory factor (LIF), family with sequence similarity 3 member C (FAM3C), dickkopf WNT signalling pathway inhibitor 3 (DKK3) and IL-8. These factors are able to promote metastasis via mechanisms that include MMP2 upregulation (IL-8), epithelial to mesenchymal transition (FAM3C, DKK3, LIF) and promotion of angiogenesis (IL-8 and DKK3) (83).

TLR3 and TLR4, that are expressed on immune cells including macrophages and DCS but also a variety of cancer cell, can activate the release of an array of cytokines (84). Recently, it has been demonstrated that TLR3 and TLR4 activation (in an LPS or poly(I:C) induced model) in lung cancer cell lines (A549 and H460) causes 1) Lys63 linked ubiquitynation of TNF receptor associated factor 6 (TRAF6) and 2)induced autophagy. Herein, autophagy was required for TRAF6 ubiquitination that leads to downstream activation of NFkB and MAPK signalling and subsequent cytokine production. As a result, autophagy deficiency in these lung cancer cells reduced release of IL-6, C-C motif chemokine ligand (CCL)-2, and CCL-20. CCL-2 secretion is associated with cell migration and CCL-20 with a metastatic phenotype. Accordingly, autophagy deficiency impaired migratory capacity. IL-6 can induce VEGFA and MMP release that are associated with an invasive phenotype. Indeed, the invasive phenotype was dependent on autophagy and IL-6 and associated with VEGFA and MMP2 release (85).

In breast cancer autophagy inhibition through ATG7 or Beclin1 knockdown altered IL-6 secretion. Interestingly, autophagy deficiency increased IL-6 secretion by MCF7 (low autophagy-dependent survival) and decreased IL-6 secretion by MDA-MB-468 (high autophagy-dependent survival) cells. IL-6 secretion is important for cancer stem cell (CSC) maintenance and is sufficient to induce CD44+:CD24low/- phenotype in breast cancer cells (86). In line, autophagy deficiency decreased mammosphere formation capacity of MDA-MB-468 cells. Rescue experiments illustrated that mammosphere formation was improved by IL-6 treatment and conditioned media from autophagy proficient MDA-MB-468 cells. Autophagy-dependent secretion of IL-6, but also other factors, are able to promote mammosphere formation and may be important in CSC maintenance (87).

\section{III.b. Pro-metastatic: Weibel-Palade body formation in vascular endothelium to facilitate metastasis}

Aberrant signaling in tumour-associated endothelial cells contributes to excessive neovascularization that is a feature of solid tumours (reviewed in (75)). As discussed above, autophagy is important to sustain the secretion of protein-containing WPB by proper 
VWF maturation. In tumour endothelial cells (TECS) these WPBs contain important secretory factors that can influence tumour progression. In line, P-selectin is sorted into WPBs as a result of its ability to interact with VWF and is translocated to the cell membrane upon stimulation (88). Once localized at the luminal side of the endothelial cell, it facilitates metastasis formation by promoting adhesion of circulating tumour cells (89). Impairment of autophagy may therefore reduce development of metastases. In addition to VWF and P-selectin, WPBs can contain other secretory proteins as angiopoietin 2 which is positively associated with tumour progression (due to its angiogenic potential) and interleukin 8 which is important in tumour progression and metastasis (due to its angiogenic and immune response modulating potential). Collectively, autophagydependent WPB formation may facilitate tumour progression, although these aspects require further investigation.

\section{Therapy resistance}

Autophagy has been implicated in promoting chemo- and radioresistance. Although often presumed to be caused by its degradative feature, we demonstrated in irradiated cancer cells that knockdown of ATG7 or LC3B, but not treatment with lysosomal inhibitor chloroquine, sensitizes cancer cells to radiation (90). This further supports a role of autophagy-related proteins to promote radioresistance through an alternative process such as secretion. For example, HMGB1 is secreted through autophagy-dependent mechanisms during ICD $(11,71)$. Although this factor is an important DAMP that can increase immunogenic responses, HMGB1 increases Doxorubicin resistance in neighboring breast cancer cells (91). In line, the interaction of HMGB1 with the receptor for advanced glycation end products (RAGE) that is expressed on various cell lines in the tumour increases chemo resistance by inducing pro-survival autophagy (92).

In addition to increasing angiogenesis and the pro-metastatic potential by DKK3 DKK3 expression is associated with docetaxel chemo sensitivity in lung cancer cells through decreasing expression of the drug efflux pump P-glycoprotein (93). Furthermore, DKK3 overexpression in an esophageal adenocarcinoma cell line was associated with increased 5-FU and cisplatin resistance, invasion and activation of the TGF-B signaling (94).

In short, autophagy-dependent secretion is involved in anti-tumour effects through enhancing immunosurveillance, but is also important in tumour progression through stimulation of angiogenesis, changing drug resistance, triggering EMT and increasing metastases development. Manipulation of the secreted arsenal of proteins, and tilting the balance more towards an anti-tumour strategy may be an attractive novel approach in cancer treatment. 
Table 2 autophagy dependent secreted factors

\begin{tabular}{|c|c|c|}
\hline Protein & Mechanism & REF \\
\hline Acbp & Atg5, Atg7, Atg8, Atg12 & $(16)$ \\
\hline IL-1B & ATG5 & $(11)$ \\
\hline NBR1, P62, OPTN and CACO2 LC3B-II & Inhibition or depletion of PIKfyve by apilimod or siRNA & (19) \\
\hline angiogenin 4 , interlectin 1 and relm $\beta$ & ATG16L1 ATG5 or ATG7 & $(25)$ \\
\hline MUC5AC & ATG5 and ATG16L1 & $(26)$ \\
\hline Insulin & GABARAP & \\
\hline NPY & ATG16L1 as RAB33a effector & (33) \\
\hline $\begin{array}{l}\text { von Willebrand factor ( } \mathrm{vWF} \text { ), P-selectin, } \\
\text { interleukin-8, angiopoietin-2 and endothelin-1 }\end{array}$ & ATG7,ATG5, chloroquine or bafilomycin A1 & (34) \\
\hline matrix-degrading factors including cathepsin $\mathrm{K}$ & ATG5 or ATG7 & (35) \\
\hline
\end{tabular}

\section{Autophagy-dependent receptor trafficking in tumour progression}

Autophagy execution requires cargo recognition, packaging, vesicle transport, vesicle fusion and degradation. In addition to the catabolic function of autophagy, the autophagy machinery is utilized for more purposes including intracellular trafficking and endocytic signaling. In addition to these roles in secretion, autophagy mediates the retroand anterograde trafficking of membrane bound receptors that may influence tumour progression.

For example, the GABARAP protein family mediates membrane of cell- surface expression of receptors like the GABA (A) receptor (GABAAR (95), the human kappa opioid receptor (hKOPR) (96), transient receptor potential cation channel subfamily $\vee$ member 1 (TRPV1) (97), the angiotensin II receptors (AGTR) $(98,99)$ and the epidermal growth factor receptor (EGFR) (100).

EGFR controls cell proliferation, migration and differentiation and is frequently over activated in several cancer types due to amplification or mutation (101). EGFR expressing tumours depend on autophagy for their survival and proliferation. Inhibition of autophagy by the administration of chloroquine abrogated the radioresistant phenotype of these tumours (55)(reviewed in (102)). Interestingly, during hypoxia translocation of EGFR to the plasma membrane is controlled by GABARAPL1 (100). Upon hypoxia exposure, GABARAPL1 colocalizes with EGFR at the cytoplasmic site of the plasma membrane. Moreover, knockdown of GABARAPL1 resulted in a decrease in EGFR membrane expression, but not in overall EGFR expression, suggesting a role for GABARAPL1 in anterograde transport of EGFR.

The KOR and GABAAR are involved in neurological processes and play a role in a variety of processes like pain sensation, consciousness, and mood.

The GABA(A) receptor (GABAAR) is well known for its inhibitory role on active neurons and is expressed on the postsynaptic throughout the whole body, although mainly expressed in the mammalian brain. Surprisingly, overexpression of the GABAAR leads to 
several types of cancer including breast, liver, lung and pancreatic cancers and contributes to migration of breast cancer cells through activation of extracellular-regulated kinase 1/2 (ERK1/2) (103-108). GABARAP and GABARAPL1 are both involved in GABAAR trafficking towards the plasma membrane. In this role, GABARAP probably serves as a cargo-receptor which mediates GABAAR incorporation in the transport vesicle by a direct interaction with the $\gamma 2$ subunit of the receptor. ABARAP knockdown mice show no defects in GABAAR expression, suggesting that GABARAP is redundant and other molecules, like its homolog GABARAPL1, can take over its function (109).

In contrast to the pro-tumorigenic effects of EGFR and GABAAR signalling, membrane expression mediated through autophagy-related proteins also results in the expression of receptors that may inhibit tumour progression, for example through KOR signalling. The KOR is well characterized for its analgesic role. However, the KOR also acts as a negative regulator of cell proliferation in breast, lung and prostate cancers $(110,111)$. Opioid receptors belong to the GPCR family, and activation of the receptors modulates the MAPK pathway and inhibit pro survival PI3K/AKT signalling molecules and may antagonize EGFR signalling (110). Both GABARAP and GABARAPL1 are required for anterograde transport of the KOR receptor. Interestingly, because of its stronger interaction GABARAPL1 does not need C-terminal modification in contrast to GABARAP, which requires membrane association to transport the KOR to the plasma membrane (96).

Taken together, the GABARAP family proteins mediates trafficking and surface expression of receptors with both tumour promoting (EGFR, GABAAR) and tumour inhibitory characteristics (KOR). This suggests that the GABARAP family contributes to cancer progression in a context-dependent manner, being in a tumour promoting or inhibitory role.

\section{Concluding remarks}

Autophagy has been considered as an important tumour suppressive process for cellular homeostasis by effectuating lysosomal degradation of the cells' toxic constituents. Importantly autophagy mediates an additional cellular feature, the trafficking and release of specific proteins. These effects are important during physiological conditions (e.g. maintaining a barrier for infection by mucus and lysozyme secretion and waste secretion), but also mediate important effects in tumour progression (figure 1). The autophagy mediated secreted factors may stimulate cellular proliferation via auto- and paracrine signaling and establish a communicative tool between cells that can either stimulate or limit tumour progression. The autophagy mediated release of DAMPs seem to be ICD inducer-dependent and polarize the tumour microenvironment towards a less immunosuppressive phenotype. Alternatively, tumours are characterized by promoting an immunosuppressed tumour microenvironment by cytokine signaling. Furthermore, autophagy mediated secretory signaling promotes an invasive phenotype. 


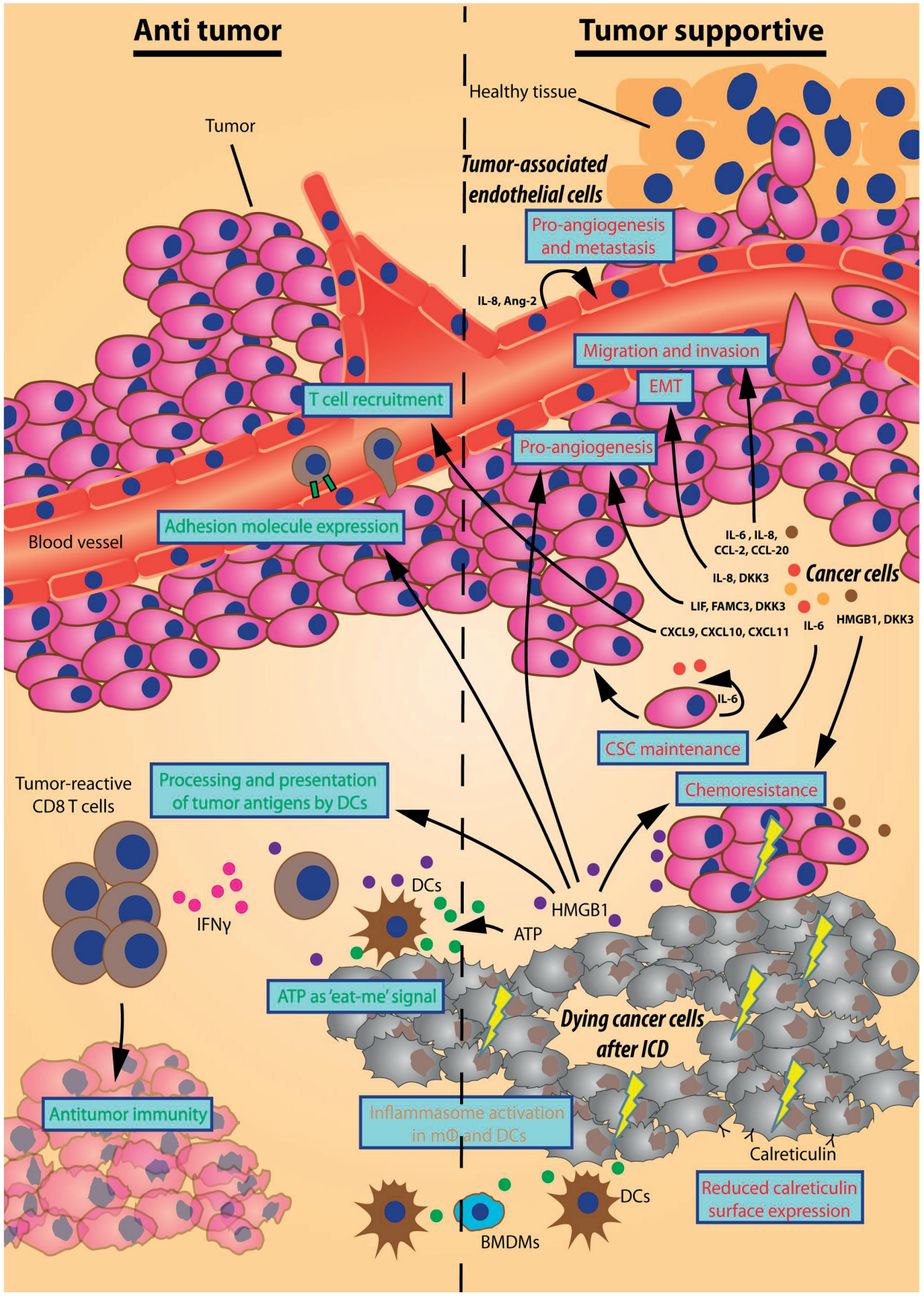

Figure 1. Effects of autophagy dependent secretion on tumour progression.

Schematic representation of autophagy mediated secretory events that either inhibit or support tumour progression displayed here on the left ('Anti tumour') and right side ('Tumour supportive') respectively. The different sources of autophagy dependent secretory factors (bold italic) establish multiple effects on the 
tumour microenvironment (in blue boxes) by the designated factors. As such, factors that promote angiogenesis, invasion, a migratory phenotype, cancer stem cell (CSC) maintenance or chemoresistance support tumour progression. Also, a reduced surface expression of calreticulin by cancer cells undergoing immunogenic cell death (ICD) hinders an immunogenic response. Oppositely, some factors have counteractive effects on tumour progression by improving immune cell adhesion or recruitment. Moreover, the 'eat-me' signal ATP together with HMGB1/TLR4 mediated improved processing and presentation of tumour antigens by dendritic cells (DCS) promote interferon gamma (IFNY) producing T cells to aid antitumor immunity. The ATP/P2RX7mediated activation of the inflammasome in macrophages $(m \phi)$ and DCs can have an array of effects of which the final inhibition/support of tumour progression may be context-dependent.

An important note is that autophagy may convey direct or indirect effects on secretory events through formation of the mobilized vesicle, facilitation of anterograde trafficking or alterations in homeostasis and/or autonomous cell signaling.

In conclusion, autophagy (or autophagy-related proteins) is an important cellular process that is more elaborate than solemnly a degradative pathway. It facilitates multiple secretory events that can promote tumour progression by limiting immunosurveillance and stimulating invasiveness and angiogenesis.

\section{Acknowledgements}

This work was financially supported by the Dutch Cancer Society (KWF Grants UM 20125506 and 2015-7735 to K.R.), worldwide cancer research award 16-0265 (to K.R.) and the FWO Postdoctoral Fellowship program (to M.S.) 


\section{References}

1. Rubinsztein DC, Shpilka T, Elazar Z. Mechanisms of autophagosome biogenesis. Curr Biol 2012;22:R29-34

2. Schaaf MB, Keulers TG, Vooijs MA, Rouschop KM. LC3/GABARAP family proteins: autophagy-(un)related functions. Faseb J 2016

3. Rouschop KM, van den Beucken T, Dubois L, Niessen H, Bussink J, Savelkouls K, et al. The unfolded protein response protects human tumor cells during hypoxia through regulation of the autophagy genes MAP1LC3B and ATG5. J Clin Invest 2010;120:127-41

4. Mathew R, Karantza-Wadsworth V, White E. Role of autophagy in cancer. Nat Rev Cancer 2007;7:961-7

5. Harris AL. Hypoxia--a key regulatory factor in tumour growth. Nat Rev Cancer 2002;2:38-47

6. Lee MC, Miller EA, Goldberg J, Orci L, Schekman R. Bi-directional protein transport between the ER and Golgi. Annual review of cell and developmental biology 2004;20:87-123

7. Ponnambalam S, Baldwin SA. Constitutive protein secretion from the trans-Golgi network to the plasma membrane. Molecular membrane biology 2003;20:129-39

8. Rabouille C, Malhotra V, Nickel W. Diversity in unconventional protein secretion. Journal of cell science 2012;125:5251-5

9. McGrath JP, Varshavsky A. The yeast STE6 gene encodes a homologue of the mammalian multidrug resistance P-glycoprotein. Nature 1989;340:400-4

10. Steringer JP, Bleicken S, Andreas H, Zacherl S, Laussmann M, Temmerman K, et al. Phosphatidylinositol 4,5-bisphosphate ( $\mathrm{PI}(4,5) \mathrm{P} 2)$-dependent oligomerization of fibroblast growth factor 2 (FGF2) triggers the formation of a lipidic membrane pore implicated in unconventional secretion. The Journal of biological chemistry 2012;287:27659-69

11. Dupont N, Jiang S, Pilli M, Ornatowski W, Bhattacharya D, Deretic V. Autophagy-based unconventional secretory pathway for extracellular delivery of IL-1beta. EMBO J 2011;30:4701-11

12. Pallet N, Sirois I, Bell C, Hanafi LA, Hamelin K, Dieude M, et al. A comprehensive characterization of membrane vesicles released by autophagic human endothelial cells. Proteomics 2013;13:1108-20

13. Sirois I, Groleau J, Pallet N, Brassard N, Hamelin K, Londono I, et al. Caspase activation regulates the extracellular export of autophagic vacuoles. Autophagy 2012;8:927-37

14. Ponpuak M, Mandell MA, Kimura T, Chauhan S, Cleyrat C, Deretic V. Secretory autophagy. Current opinion in cell biology 2015;35:106-16

15. Fader CM, Colombo MI. Autophagy and multivesicular bodies: two closely related partners. Cell death and differentiation 2009;16:70-8

16. Duran JM, Anjard C, Stefan C, Loomis WF, Malhotra V. Unconventional secretion of Acb1 is mediated by autophagosomes. J Cell Biol 2010;188:527-36

17. Jang DJ, Lee JA. The roles of phosphoinositides in mammalian autophagy. Arch Pharm Res 2016;39:1129-36

18. Burman C, Ktistakis NT. Regulation of autophagy by phosphatidylinositol 3-phosphate. FEBS Lett 2010;584:1302-12

19. Hessvik NP, Øverbye A, Brech A, Torgersen ML, Jakobsen IS, Sandvig K, et al. PIKfyve inhibition increases exosome release and induces secretory autophagy. Cellular and molecular life sciences : CMLS 2016

20. Zhang Z, Xu X, Ma J, Wu J, Wang Y, Zhou R, et al. Gene deletion of Gabarap enhances NIrp3 inflammasome-dependent inflammatory responses. J Immunol 2013;190:3517-24

21. Zhang M, Kenny SJ, Ge L, Xu K, Schekman R. Translocation of interleukin-1beta into a vesicle intermediate in autophagy-mediated secretion. eLife 2015;4

22. Cadwell K, Liu JY, Brown SL, Miyoshi H, Loh J, Lennerz JK, et al. A key role for autophagy and the autophagy gene Atg16l1 in mouse and human intestinal Paneth cells. Nature 2008;456:259-63

23. Cadwell K, Patel KK, Komatsu M, Virgin HWt, Stappenbeck TS. A common role for Atg16L1, Atg5 and Atg7 in small intestinal Paneth cells and Crohn disease. Autophagy 2009;5:250-2

24. Hampe J, Franke A, Rosenstiel P, Till A, Teuber M, Huse K, et al. A genome-wide association scan of nonsynonymous SNPs identifies a susceptibility variant for Crohn disease in ATG16L1. Nature genetics 2007;39:207-11 
25. Tsuboi K, Nishitani M, Takakura A, Imai Y, Komatsu M, Kawashima H. Autophagy Protects against Colitis by the Maintenance of Normal Gut Microflora and Secretion of Mucus. The Journal of biological chemistry 2015;290:20511-26

26. Dickinson JD, Alevy Y, Malvin NP, Patel KK, Gunsten SP, Holtzman MJ, et al. IL13 activates autophagy to regulate secretion in airway epithelial cells. Autophagy 2016;12:397-409

27. Conway KL, Kuballa P, Song JH, Patel KK, Castoreno AB, Yilmaz OH, et al. Atg16l1 is required for autophagy in intestinal epithelial cells and protection of mice from Salmonella infection. Gastroenterology 2013;145:1347-57

28. von Muhlinen N, Akutsu M, Ravenhill BJ, Foeglein A, Bloor S, Rutherford TJ, et al. LC3C, bound selectively by a noncanonical LIR motif in NDP52, is required for antibacterial autophagy. Molecular cell 2012;48:329-42

29. Weidberg H, Shvets E, Shpilka T, Shimron F, Shinder V, Elazar Z. LC3 and GATE-16/GABARAP subfamilies are both essential yet act differently in autophagosome biogenesis. The EMBO journal 2010;29:1792-802

30. Coyle JE, Qamar S, Rajashankar KR, Nikolov DB. Structure of GABARAP in two conformations: implications for GABA(A) receptor localization and tubulin binding. Neuron 2002;33:63-74

31. Nath S, Dancourt J, Shteyn V, Puente G, Fong WM, Nag S, et al. Lipidation of the LC3/GABARAP family of autophagy proteins relies on a membrane-curvature-sensing domain in Atg3. Nature cell biology 2014;16:415-24

32. Asano S, Nemoto T, Kitayama T, Harada K, Zhang J, Harada K, et al. Phospholipase C-related catalytically inactive protein (PRIP) controls KIF5B-mediated insulin secretion. Biology open 2014;3:463-74

33. Ishibashi K, Uemura T, Waguri S, Fukuda M. Atg16L1, an essential factor for canonical autophagy, participates in hormone secretion from PC12 cells independently of autophagic activity. Molecular biology of the cell 2012;23:3193-202

34. Torisu T, Torisu K, Lee IH, Liu J, Malide D, Combs CA, et al. Autophagy regulates endothelial cell processing, maturation and secretion of von Willebrand factor. Nature medicine 2013;19:1281-7

35. DeSelm CJ, Miller BC, Zou W, Beatty WL, van Meel E, Takahata Y, et al. Autophagy proteins regulate the secretory component of osteoclastic bone resorption. Developmental cell 2011;21:966-74

36. Vogiatzi T, Xilouri M, Vekrellis K, Stefanis L. Wild type alpha-synuclein is degraded by chaperonemediated autophagy and macroautophagy in neuronal cells. The Journal of biological chemistry 2008;283:23542-56

37. Ejlerskov P, Rasmussen I, Nielsen TT, Bergström A-LL, Tohyama Y, Jensen PH, et al. Tubulin polymerization-promoting protein (TPPP/p2 $5 \alpha)$ promotes unconventional secretion of-synuclein through exophagy by impairing autophagosome-lysosome fusion. The Journal of biological chemistry 2013;288:17313-35

38. Nilsson $\mathrm{P}$, Loganathan $\mathrm{K}$, Sekiguchi $\mathrm{M}$, Matsuba $\mathrm{Y}$, Hui $\mathrm{K}$, Tsubuki $\mathrm{S}$, et al. A $\beta$ secretion and plaque formation depend on autophagy. Cell Rep 2013;5:61-9

39. Sullivan LB, Chandel NS. Mitochondrial reactive oxygen species and cancer. Cancer \& metabolism $2014 ; 2: 17$

40. Phinney DG, Di Giuseppe M, Njah J, Sala E, Shiva S, St Croix CM, et al. Mesenchymal stem cells use extracellular vesicles to outsource mitophagy and shuttle microRNAs. Nat Commun 2015;6:8472

41. Unuma K, Aki T, Funakoshi T, Hashimoto K, Uemura K. Extrusion of mitochondrial contents from lipopolysaccharide-stimulated cells: Involvement of autophagy. Autophagy 2015;11:1520-36

42. Sirois I, Groleau J, Pallet N, Brassard N, Hamelin K, Londono I, et al. Caspase activation regulates the extracellular export of autophagic vacuoles. Autophagy 2012;8:877-6

43. Pallet N, Sirois I, Bell C, Hanafi L-AA, Hamelin K, Dieudé M, et al. A comprehensive characterization of membrane vesicles released by autophagic human endothelial cells. Proteomics 2013;13:1108-20

44. Dewhirst MW, Cao Y, Moeller B. Cycling hypoxia and free radicals regulate angiogenesis and radiotherapy response. Nat Rev Cancer 2008;8:425-37

45. Nordsmark M, Bentzen SM, Rudat V, Brizel D, Lartigau E, Stadler P, et al. Prognostic value of tumor oxygenation in 397 head and neck tumors after primary radiation therapy. An international multi-center study. Radiother Oncol 2005;77:18-24 
46. Cao Y, Klionsky DJ. Physiological functions of Atg6/Beclin 1: a unique autophagy-related protein. Cell research 2007;17:839-49

47. Qu X, Yu J, Bhagat G, Furuya N, Hibshoosh H, Troxel A, et al. Promotion of tumorigenesis by heterozygous disruption of the beclin 1 autophagy gene. J Clin Invest 2003;112:1809-20

48. Yue Z, Jin S, Yang C, Levine AJ, Heintz N. Beclin 1, an autophagy gene essential for early embryonic development, is a haploinsufficient tumor suppressor. Proc Natl Acad Sci U S A 2003;100:15077-82

49. Wei H, Wei S, Gan B, Peng X, Zou W, Guan JL. Suppression of autophagy by FIP200 deletion inhibits mammary tumorigenesis. Genes \& development 2011;25:1510-27

50. Rouschop KM, Ramaekers CH, Schaaf MB, Keulers TG, Savelkouls KG, Lambin P, et al. Autophagy is required during cycling hypoxia to lower production of reactive oxygen species. Radiother Oncol 2009;92:411-6

51. Rouschop KM, van den Beucken T, Dubois L, Niessen H, Bussink J, Savelkouls K, et al. The unfolded protein response protects human tumor cells during hypoxia through regulation of the autophagy genes MAP1LC3B and ATG5. J Clin Invest 2010;120:127-41

52. Schaaf MB, Cojocari D, Keulers TG, Jutten B, Starmans MH, de Jong MC, et al. The autophagy associated gene, ULK1, promotes tolerance to chronic and acute hypoxia. Radiotherapy and oncology : journal of the European Society for Therapeutic Radiology and Oncology 2013;108:529-34

53. Pike LR, Singleton DC, Buffa F, Abramczyk O, Phadwal K, Li JL, et al. Transcriptional up-regulation of ULK1 by ATF4 contributes to cancer cell survival. The Biochemical journal 2013;449:389-400

54. Keulers TG, Schaaf MB, Peeters HJ, Savelkouls KG, Vooijs MA, Bussink J, et al. GABARAPL1 is required for increased EGFR membrane expression during hypoxia. Radiother Oncol 2015;116:417-22

55. Jutten B, Keulers TG, Schaaf MB, Savelkouls K, Theys J, Span PN, et al. EGFR overexpressing cells and tumors are dependent on autophagy for growth and survival. Radiotherapy and oncology : journal of the European Society for Therapeutic Radiology and Oncology 2013;108:479-83

56. Eng CH, Wang Z, Tkach D, Toral-Barza L, Ugwonali S, Liu S, et al. Macroautophagy is dispensable for growth of KRAS mutant tumors and chloroquine efficacy. Proc Natl Acad Sci U S A 2016;113:182-7

57. Guo JY, Chen HY, Mathew R, Fan J, Strohecker AM, Karsli-Uzunbas G, et al. Activated Ras requires autophagy to maintain oxidative metabolism and tumorigenesis. Genes \& development 2011;25:460-70

58. Lock R, Kenific CM, Leidal AM, Salas E, Debnath J. Autophagy-dependent production of secreted factors facilitates oncogenic RAS-driven invasion. Cancer discovery 2014;4:466-79

59. Garg AD, Galluzzi L, Apetoh L, Baert T, Birge RB, Bravo-San Pedro JM, et al. Molecular and Translational Classifications of DAMPs in Immunogenic Cell Death. Frontiers in immunology 2015;6:588

60. Garg AD, Nowis D, Golab J, Vandenabeele P, Krysko DV, Agostinis P. Immunogenic cell death, DAMPs and anticancer therapeutics: an emerging amalgamation. Biochimica et biophysica acta 2010;1805:53-71

61. Ray-Coquard I, Cropet C, Van Glabbeke M, Sebban C, Le Cesne A, Judson I, et al. Lymphopenia as a prognostic factor for overall survival in advanced carcinomas, sarcomas, and lymphomas. Cancer research 2009;69:5383-91

62. Garg AD, De Ruysscher D, Agostinis P. Immunological metagene signatures derived from immunogenic cancer cell death associate with improved survival of patients with lung, breast or ovarian malignancies: A large-scale meta-analysis. Oncoimmunology 2016;5:e1069938

63. Ko A, Kanehisa A, Martins I, Senovilla L, Chargari C, Dugue D, et al. Autophagy inhibition radiosensitizes in vitro, yet reduces radioresponses in vivo due to deficient immunogenic signalling. Cell death and differentiation 2014;21:92-9

64. Michaud M, Martins I, Sukkurwala AQ, Adjemian S, Ma Y, Pellegatti P, et al. Autophagy-dependent anticancer immune responses induced by chemotherapeutic agents in mice. Science 2011;334:1573-7

65. Martins I, Wang Y, Michaud M, Ma Y, Sukkurwala AQ, Shen S, et al. Molecular mechanisms of ATP secretion during immunogenic cell death. Cell death and differentiation 2014;21:79-91

66. Michaud M, Xie X, Bravo-San Pedro JM, Zitvogel L, White E, Kroemer G. An autophagy-dependent anticancer immune response determines the efficacy of melanoma chemotherapy. Oncoimmunology 2014;3:e944047 
67. Pietrocola F, Pol J, Vacchelli E, Rao S, Enot DP, Baracco EE, et al. Caloric Restriction Mimetics Enhance Anticancer Immunosurveillance. Cancer cell 2016;30:147-60

68. Garg AD, Dudek AM, Ferreira GB, Verfaillie T, Vandenabeele P, Krysko DV, et al. ROS-induced autophagy in cancer cells assists in evasion from determinants of immunogenic cell death. Autophagy 2013;9:1292-307

69. Garg AD, Dudek AM, Agostinis P. Calreticulin surface exposure is abrogated in cells lacking, chaperonemediated autophagy-essential gene, LAMP2A. Cell death \& disease 2013;4:e826

70. Garg AD, Krysko DV, Vandenabeele P, Agostinis P. Extracellular ATP and P(2)X(7) receptor exert contextspecific immunogenic effects after immunogenic cancer cell death. Cell death \& disease 2016;7:e2097

71. Thorburn J, Horita H, Redzic J, Hansen K, Frankel AE, Thorburn A. Autophagy regulates selective HMGB1 release in tumor cells that are destined to die. Cell death and differentiation 2009;16:175-83

72. Apetoh L, Ghiringhelli F, Tesniere A, Criollo A, Ortiz C, Lidereau R, et al. The interaction between HMGB1 and TLR4 dictates the outcome of anticancer chemotherapy and radiotherapy. Immunological reviews 2007;220:47-59

73. Sachdev U, Cui X, Hong G, Namkoong S, Karlsson JM, Baty CJ, et al. High mobility group box 1 promotes endothelial cell angiogenic behavior in vitro and improves muscle perfusion in vivo in response to ischemic injury. Journal of vascular surgery 2012;55:180-91

74. van Beijnum JR, Nowak-Sliwinska P, van den Boezem E, Hautvast P, Buurman WA, Griffioen AW. Tumor angiogenesis is enforced by autocrine regulation of high-mobility group box 1 . Oncogene 2013;32:363-74

75. Maes H, Olmeda D, Soengas MS, Agostinis P. Vesicular trafficking mechanisms in endothelial cells as modulators of the tumor vasculature and targets of antiangiogenic therapies. The FEBS journal 2016;283:25-38

76. Garg AD, Vandenberk L, Koks C, Verschuere T, Boon L, Van Gool SW, et al. Dendritic cell vaccines based on immunogenic cell death elicit danger signals and T cell-driven rejection of high-grade glioma. Science translational medicine 2016;8:328ra27

77. Ghiringhelli F, Apetoh L, Tesniere A, Aymeric L, Ma Y, Ortiz C, et al. Activation of the NLRP3 inflammasome in dendritic cells induces IL-1beta-dependent adaptive immunity against tumors. Nature medicine 2009;15:1170-8

78. Takenouchi T, Nakai M, Iwamaru Y, Sugama S, Tsukimoto M, Fujita M, et al. The activation of P2X7 receptor impairs lysosomal functions and stimulates the release of autophagolysosomes in microglial cells. Journal of immunology (Baltimore, Md : 1950) 2009;182:2051-62

79. Qin Y, Ekmekcioglu S, Liu P, Duncan LM, Lizee G, Poindexter N, et al. Constitutive aberrant endogenous interleukin-1 facilitates inflammation and growth in human melanoma. Molecular cancer research : MCR 2011;9:1537-50

80. Coffelt SB, Kersten K, Doornebal CW, Weiden J, Vrijland K, Hau CS, et al. IL-17-producing gammadelta T cells and neutrophils conspire to promote breast cancer metastasis. Nature 2015;522:345-8

81. Salah FS, Ebbinghaus M, Muley VY, Zhou Z, Al-Saadi KR, Pacyna-Gengelbach M, et al. Tumor suppression in mice lacking GABARAP, an Atg8/LC3 family member implicated in autophagy, is associated with alterations in cytokine secretion and cell death. Cell death \& disease 2016; 7:e2205

82. Fisher DT, Appenheimer MM, Evans SS. The two faces of IL- 6 in the tumor microenvironment. Seminars in immunology 2014;26:38-47

83. Kraya AA, Piao S, Xu X, Zhang G, Herlyn M, Gimotty P, et al. Identification of secreted proteins that reflect autophagy dynamics within tumor cells. Autophagy 2015;11:60-74

84. Kawai T, Akira S. The role of pattern-recognition receptors in innate immunity: update on Toll-like receptors. Nature immunology 2010;11:373-84

85. Zhan Z, Xie X, Cao H, Zhou X, Zhang XD, Fan H, et al. Autophagy facilitates TLR4- and TLR3-triggered migration and invasion of lung cancer cells through the promotion of TRAF6 ubiquitination. Autophagy 2014;10:257-68

86. Iliopoulos D, Hirsch HA, Wang G, Struhl K. Inducible formation of breast cancer stem cells and their dynamic equilibrium with non-stem cancer cells via IL6 secretion. Proc Natl Acad Sci U S A 2011;108:1397-402 
87. Maycotte P, Jones KL, Goodall ML, Thorburn J, Thorburn A. Autophagy Supports Breast Cancer Stem Cell Maintenance by Regulating IL6 Secretion. Molecular cancer research : MCR 2015;13:651-8

88. Hop C, Guilliatt A, Daly M, de Leeuw HP, Brinkman HJ, Peake IR, et al. Assembly of multimeric von Willebrand factor directs sorting of P-selectin. Arteriosclerosis, thrombosis, and vascular biology 2000;20:1763-8

89. Laubli H, Borsig L. Selectins promote tumor metastasis. Seminars in cancer biology 2010;20:169-77

90. Schaaf MB, Jutten B, Keulers TG, Savelkouls KG, Peeters HJ, van den Beucken T, et al. Canonical autophagy does not contribute to cellular radioresistance. Radiother Oncol 2015;114:406-12

91. Amornsupak K, Insawang T, Thuwajit P, P OC, Eccles SA, Thuwajit C. Cancer-associated fibroblasts induce high mobility group box 1 and contribute to resistance to doxorubicin in breast cancer cells. BMC cancer 2014;14:955

92. Kang R, Tang D, Schapiro NE, Livesey KM, Farkas A, Loughran P, et al. The receptor for advanced glycation end products (RAGE) sustains autophagy and limits apoptosis, promoting pancreatic tumor cell survival. Cell death and differentiation 2010;17:666-76

93. Tao L, Huang G, Chen Y, Chen L. DNA methylation of DKK3 modulates docetaxel chemoresistance in human nonsmall cell lung cancer cell. Cancer biotherapy \& radiopharmaceuticals 2015;30:100-6

94. Wang Z, Lin L, Thomas DG, Nadal E, Chang AC, Beer DG, et al. The role of Dickkopf-3 overexpression in esophageal adenocarcinoma. The Journal of thoracic and cardiovascular surgery 2015;150:377-85 e2

95. Jacob TC, Moss SJ, Jurd R. GABA(A) receptor trafficking and its role in the dynamic modulation of neuronal inhibition. Nat Rev Neurosci 2008;9:331-43

96. Chen C, Wang Y, Huang P, Liu-Chen LY. Effects of C-terminal modifications of GEC1 protein and gammaaminobutyric acid type $A(G A B A(A))$ receptor-associated protein (GABARAP), two microtubule-associated proteins, on kappa opioid receptor expression. The Journal of biological chemistry 2011;286:15106-15

97. Lainez S, Valente P, Ontoria-Oviedo I, Estevez-Herrera J, Camprubi-Robles M, Ferrer-Montiel A, et al. GABAA receptor associated protein (GABARAP) modulates TRPV1 expression and channel function and desensitization. Faseb J 2010;24:1958-70

98. Cook JL, Re RN, deHaro DL, Abadie JM, Peters M, Alam J. The trafficking protein GABARAP binds to and enhances plasma membrane expression and function of the angiotensin II type 1 receptor. Circ Res 2008;102:1539-47

99. Alam J, Deharo D, Redding KM, Re RN, Cook JL. C-terminal processing of GABARAP is not required for trafficking of the angiotensin II type 1A receptor. Regul Pept 2010;159:78-86

100. Keulers TG, Schaaf MB, Peeters HJ, Savelkouls KG, Vooijs MA, Bussink J, et al. GABARAPL1 is required for increased EGFR membrane expression during hypoxia. Radiotherapy and oncology : journal of the European Society for Therapeutic Radiology and Oncology 2015;116:417-22

101. Theys J, Jutten B, Dubois L, Rouschop KM, Chiu RK, Li Y, et al. The deletion mutant EGFRvIII significantly contributes to stress resistance typical for the tumour microenvironment. Radiotherapy and oncology : journal of the European Society for Therapeutic Radiology and Oncology 2009;92:399-404

102. Jutten B, Rouschop KM. EGFR signaling and autophagy dependence for growth, survival, and therapy resistance. Cell Cycle 2014;13:42-51

103. Sizemore GM, Sizemore ST, Seachrist DD, Keri RA. GABA(A) receptor pi (GABRP) stimulates basal-like breast cancer cell migration through activation of extracellular-regulated kinase 1/2 (ERK1/2). The Journal of biological chemistry 2014;289:24102-13

104. Li YH, Liu Y, Li YD, Liu YH, Li F, Ju Q, et al. GABA stimulates human hepatocellular carcinoma growth through overexpressed GABAA receptor theta subunit. World J Gastroenterol 2012;18:2704-11

105. Liu Y, Li YH, Guo FJ, Wang JJ, Sun RL, Hu JY, et al. Gamma-aminobutyric acid promotes human hepatocellular carcinoma growth through overexpressed gamma-aminobutyric acid A receptor alpha 3 subunit. World J Gastroenterol 2008;14:7175-82

106. Schuller HM, Al-Wadei HA. Beta-adrenergic signaling in the development and progression of pulmonary and pancreatic adenocarcinoma. Curr Cancer Ther Rev 2012;8:116-27 
107. Takehara A, Hosokawa M, Eguchi H, Ohigashi H, Ishikawa O, Nakamura Y, et al. Gamma-aminobutyric acid (GABA) stimulates pancreatic cancer growth through overexpressing GABAA receptor pi subunit. Cancer research 2007;67:9704-12

108. Wu W, Yang Q, Fung KM, Humphreys MR, Brame LS, Cao A, et al. Linking gamma-aminobutyric acid A receptor to epidermal growth factor receptor pathways activation in human prostate cancer. Mol Cell Endocrinol 2014;383:69-79

109. O'Sullivan GA, Kneussel M, Elazar Z, Betz H. GABARAP is not essential for GABA receptor targeting to the synapse. Eur J Neurosci 2005;22:2644-8

110. Kharmate G, Rajput PS, Lin YC, Kumar U. Inhibition of tumor promoting signals by activation of SSTR2 and opioid receptors in human breast cancer cells. Cancer Cell Int 2013;13:93

111. Panagiotou S, Bakogeorgou E, Papakonstanti E, Hatzoglou A, Wallet F, Dussert C, et al. Opioid agonists modify breast cancer cell proliferation by blocking cells to the $\mathrm{G} 2 / \mathrm{M}$ phase of the cycle: involvement of cytoskeletal elements. J Cell Biochem 1999;73:204-11 


\section{Chapter}

\section{GABARAPL1 is required for the secretion of pro-angiogenic extracellular vesicles during hypoxia}

Tom G. Keulers ${ }^{1}$, Sten F. Libregts ${ }^{2}$, Marco B.E. Schaaf ${ }^{1}$, Hanneke J.M Peeters ${ }^{1}$, Kim G. Savelkouls ${ }^{1}$, Johan Bussink ${ }^{3}$, Hans Duimel ${ }^{4}$, Marijke I. Zonneveld ${ }^{1}$, Karel Bezstarosti ${ }^{5}$, Jeroen A. Demmers ${ }^{5}$, Marc Vooijs ${ }^{1}$, Marca Wauben², Kasper M.A. Rouschop ${ }^{1}$

${ }^{1}$ Maastricht Radiation Oncology (MaastRO) lab, GROW - School for Oncology and Developmental Biology, Maastricht ${ }^{2}$ University, Maastricht, the Netherlands ${ }^{2}$ Department of Biochemistry and Cell Biology, Faculty of Veterinary Medicine, ${ }^{3}$ Utrecht University, Utrecht, Netherlands. ${ }^{3}$ Department of Radiation Oncology, Radboud University Medical Center, ${ }^{4}$ Nijmegen, the Netherlands. ${ }^{4}$ Maastricht Multimodal Molecular Imaging Institute, Division of Nanoscopy, University of ${ }^{5}$ Maastricht, the Netherlands. ${ }^{5}$ Proteomics Center, Erasmus University Medical Center, Rotterdam, the Netherlands. 


\section{Abstract}

Hypoxia is a hallmark of solid tumours and is associated with tumour progression and therapy resistance. In response to hypoxia, tumour cells secrete pro-angiogenic factors to induce blood vessel formation and restore oxygen supply to the hypoxic region. Extracellular vesicles (EVs) are emerging as mediators of intercellular communication in the tumour microenvironment. Recently, we identified the LC3/GABARAP protein family member GABARAPL1 as a hypoxia-induced protein. Here, we demonstrate that GABARAPL1 is required for secretion of pro-angiogenic EVs during hypoxia. Interesting, GABARAPL1 is expressed on the EV surface and can be targeted. This results in blockade of pro-angiogenic responses in vitro. Silencing GABARAPL1 with inducible knockdown models perturbs EV secretion and results in decreased tumour growth due to decreased vascularisation and enhanced necrosis. Interestingly, targeting GABARAPL1 directly after tumour irradiation resulted in enhanced tumour regrowth delay. Implantation of control tumours at the contralateral flank of animals carrying GABARAPL1-deficient tumours restored vascularisation and tumour necrosis. This illustrates the potential systemic effects of GABARAPL1 ${ }^{+}$EVs in tumour progression. In line with the systemic effects, we demonstrate that GABARAPL1 ${ }^{+}$EVs are detectable and increased in blood of cancer patients.

In short, we reveal that hypoxic tumour cells secrete a unique EV subset, marked by GABARAPL1 expression. These EVs control tumour progression, are targetable and are therefore interesting to pursue as biomarker and therapeutic target. 


\section{Introduction}

The tumour microenvironment is characterized by extreme heterogeneity in oxygenation that primarily arises due to a poorly developed vascular network. Gradients of oxygenation in the tumour vary from normal (5\%) values around the vessel wall to complete anoxia in perinecrotic regions. Inter individual differences in tumour hypoxia largely influence outcome after therapy as high levels of tumour hypoxia correlate with therapy resistance and a more aggressive phenotype (1-4). Large differences in tumour hypoxia are observed between patients with otherwise similar clinical characteristics and are likely to be the result of the cellular ability to increase hypoxia tolerance and to resolve the hypoxic status. To this extent, cells modulate metabolism, angiogenesis, the unfolded protein response (UPR) and autophagy (5-7). The secretion of angiogenic factors (e.g. VEGF, PDGF and angiogenin) into local tissues and stroma is essential in triggering angiogenesis. These factors stimulate the adjacent quiescent vasculature to grow new vessels into the tumour and resolve hypoxia (8).

Previously, we showed that the expression of GABARAPL1, a family member of the LC3/GABARAP protein family, is induced by hypoxia and starvation due to activation of the PERK branch of the UPR (9). Members of the LC3/GABARAP protein family are associated with the lysosomal degradation pathway autophagy and intracellular receptor trafficking, they share structural characteristics, but are unique in biological functions (10).

In the recent years, exosomes and microvesicles (collectively referred to as extracelIular vesicles (EVs)) have emerged as mediators of intercellular communication. EVs are small (70-1000nm) membraned vesicles that are secreted by cells to shuttle complex biological information such as lipids, mRNA's, miRNA's, soluble and transmembrane proteins. EVs are highly enriched in tetraspanin expression (CD81, CD63 and CD9) that are widely used as markers for EV identification (11-13). It has increasingly been recognized that the total pool of secreted EVs consists of subpopulations with different marker compositions and derived from other cellular compartments (14) (15). Where these EVs are generated and what differential marker expression means in terms of biological effect, remains to be elucidated. Recent data indicates that tumour derived EVs are essential in enhancing tumour growth through stimulation of proliferation, supporting angiogenesis and influencing the immune system by triggering immunosuppressive responses (16-18). Interestingly, EVs derived from hypoxic glioblastoma cells reflect the hypoxic status of these cells and are potent inducers of angiogenesis in vitro and in vivo (19).

In this report we describe, for the first time, the discovery of a unique subset of EVs that is specifically secreted by hypoxic cells and is dependent on hypoxia-induced GABARAPL1 expression. Second, we observed that GABARAPL1 is expressed on the surface of these EVs. Exploiting this potential therapeutic entry point by using blocking antibodies against GABARAPL1, resulted in inhibition of EV function in vitro. Further- 
more we show that tumour growth and regrowth after irradiation is delayed after silencing GABARAPL1 in U87 and HT29 tumour xenografts as a result of reduced vascularization and enhanced tumour necrosis. In conclusion, we show a unique role for GABARAPL1 in tumour progression through secretion of EVs by hypoxic cells.

\section{Material \& methods}

\section{Cell culture}

HT29 (colorectal adenocarcinoma), U87 (glioblastoma) and MCF7 (breast cancer) and immortalized human umbilical cord endothelial cell lines (EC-RF-24, Applied Biological Materials, Canada) were maintained in 10\% FCS (Sigma) supplemented DMEM and RPMI (Lonza) respectively. For EV isolations, 30\% FCS was depleted from EVs by ultracentrifugation overnight (100.000 RCF). The cells' origin was authenticated by STR analysis (Identicell, Denmark). Hypoxia exposure experiments were performed using a modular atmosphere controlled system (Don Whitley Scientific). Cells were exposed to hypoxia for 16-20 hours over night. Doxycycline-inducible $(1 \mu \mathrm{g} / \mathrm{ml})$ shRNA targeting the 3'UTR of GABARAPL1 [TTACCTTACTTCATACTTGCCC] or scrambled control [CGAGGGCGACTTAACCTTAGG] was achieved through lentiviral pTRIPZ (Thermo Scientific) expression. Independent doxycycline inducible hairpins targeting the GABARAPL1 coding sequence were expressed using pLKO based lentiviral expression (ACAGTGATGAGAGTGTCTATG). GABARAPL1 knockdown was induced 3 days prior to experiments. Reintroduction of the wild type GABARAPL1 coding sequence or mutant GABARAPL1 G116A was achieved through expression in pQCXIH backbones using lentiviral transduction

\section{Mass-spectrometry}

A comprehensive description of Mass-spectrometry Sample processing, quantitative mass-spectrometry and bioinformatic analysis is provided in SI materials and methods

\section{EV purification and labeling}

EVs were isolated and purified based on the protocol described previously (20). In short, Cells were cultured in culture medium that was depleted from FCS-derived EVs by overnight ultracentrifugation at $100.000 \mathrm{~g}$. After exposure of $10 \mathrm{E}^{6} \mathrm{HT} 29$ or U87 cells to hypoxia or normoxia, EVs were collected from the culture supernatant by differential ultracentrifugation. This technique allows to isolated EVs based on their density rather than size. Samples were depleted from cellular debris and apoptotic bodies by centrifuging for $10 \mathrm{~min} 200 \mathrm{~g}$ at RT, followed by $20 \mathrm{~min} 2000 \mathrm{~g}$ and $30 \mathrm{~min}$ at $16.000 \mathrm{~g}$ and $4^{\circ} \mathrm{C}$. Finally, EVs were pelleted (60 min 100.000g) labeled with PKH67 (Sigma Aldrich) as 
described previously (21) and subsequently floated to their buoyant density into a linear sucrose gradient (0.4-2.0 M sucrose) by ultracentrifugation for 16 hours at 200.000. 12 sucrose fractions of $1 \mathrm{~mL}$ were then collected from bottom to top and either diluted with PBS for subsequent high-resolution flow cytometric analysis or immunoblotting. For plasma EV isolation, $500 \mu$ l plasma was used. Plasma was diluted with PBS and EVs were isolated by differential ultracentrifugation as described above. EVs were labeled with anti-GABARAPL1 (1:50, protein tech group) and alexa594 (1:50, Goat anti-rabbit IgG, Invitrogen) and PKH67 for general EV staining. Labeled EVs were floated into a linear sucrose gradient as described above. For ultracentrifugation SW32 and SW41 rotors (Beckman Coulter) were used. For quantification of plasma EVs, confocal microscopy analysis was performed: Fractions 6-12 were pooled and resuspended in PBS. Resuspended EVs were immobilized in 1\% agar and EV foci were counted manually. PKH67 positive foci were considered EVs. Foci lower than $3.5 \times$ backgrounds were excluded.

\section{High-Resolution Flow cytometry}

Flow cytometric analysis of individual EVs was performed on a BD Influx flow cytometer (Becton Dickinson, Brussels, Belgium) that was adapted and optimized for the analysis of single submicron-sized-particles, (21). For detection of EVs, the system was triggered on the fluorescence derived from PKH67-labeled EVs passing the first laser. The fluorescence threshold level was determined by measuring 0.22 -fiithered PBS, allowing an event rate of $\sim 10$ events/s. To allow comparisons between experiments, yellow-green fluorescent 100-nm and 200-nm polystyrene beads (FluoSpheres; Invitrogen) were used as reference. Samples were measured at event rates lower than 10.000 events/s to prevent coincident and swarm detection (22)

\section{Immunoblotting}

Cells were lysed, separated by SDS-PAGE and transferred to PVDF membranes. Sucrose gradient fractions were pooled as indicated, diluted with PBS and centrifuged 60 min and 100.000xg using SW41 rotors (Beckman Coulter). Pellets were resolved in 1x nonreducing loading buffer. Transferred proteins were probed using antibodies directed against GABARAPL1 1:1000 (11010-1-1P, Protein tech group), Actin 1:200.000 (Clone 4, MP Biomedicals), CD81 (clone JS81), CD63 (clone H5C6) and CD9 (clone ML13) 1:1500 (BD Biosciences) and MAP1LC3B 1:1500 (polyclonal, Cell Signaling), visualized using respective HRP linked secondary antibodies 1:5000 (Cell Signaling) and detected using SuperSignal West Pico Chemiluminescent Substrate (Pierce Biotechnology, Thermo Fisher Scientific). 


\section{Angiogenesis array}

Cells were exposed to normoxic and anoxic conditions. After 24 hours, culture medium was collected and centrifuged for $10 \mathrm{~min} 300 \times \mathrm{xg}$ to deplete cell debris. Angiogenesis array was performed according to the manufacturer's manual (Proteome Profiler Human Angiogenesis Array Kit, R\&D Systems).

\section{Tube formation}

Immortalized HUVEC cells were plated in 96-well plates coated with $50 \mu$ matrigel (Corning) and stimulated with EVs for 16 hours. EVs were harvested by the previously described differential ultracentrifugation from the cell culture supernatant of HT29, U87 and MCF7 control, knockdown and control cell-lines that were exposed for 16 hours to anoxia. Blocking antibody (20ng/ml. GABARAPL1 1:1000 \#11010-1-1P, Protein tech group) and EVs were incubated 30 minutes before stimulation. EGM2 medium (Lonza) was used as positive control.

\section{High resolution confocal microscopy}

After fixation (3.8\% paraformaldehyde) cells were permeabilized with $0.05 \%$ Tween in PBS and blocked using 5\% normal goat serum. Cells were probed with anti-GABARAPL1 (protein tech group, 11010-1-1P, 1:100), MAP1LC3B (polyclonal, cell signaling, 1:100), CD81 (clone JS81), CD63 (clone H5C6), CD9 (clone ML13, BD Bioscience(1:100)) and hoechst33342 (Sigma Aldrich). Goat anti-rabbit Alexa-488 (Invitrogen) and Goat antimouse A647 were used for visualization. Bound antibodies were analyzed using Leica TCS SP8 CARS confocal microscope. Images were deconvoluted using Huygens software.

\section{Tumour models}

HT29 and U87 cells were transplanted in the flanks of nu/nu mice (1E6/ in 50ul matrigel). Doxycycline was administered through the drinking water $(2 \mathrm{~g} / \mathrm{L}$ doxycycline, $5 \%$ sucrose). Tumour growth was assessed by caliper measurement. Animals were injected with pimonidazole (60 mg/kg i.p. hypoxyprobe-1; Chemicon) and BrdU (30 mg/kg i.p.; Sigma-Aldrich) 60 minutes prior to killing. All animal experiments were conducted in accordance with national guidelines and approved by the animal ethics committees of Maastricht University.

\section{Immunohistochemistry and Image Processing.}

Frozen, acetone-fixed sections were stained by using anti-pimonidazole (Chemicon), 9F1 (rat monoclonal antibody to mouse endothelium; Department of Pathology, Radboud University Nijmegen Medical Center) as described previously (23). The slides were scanned by a computerized digital image processing system by using a high-resolution 
intensified solid-state camera on a fluorescence microscope (Axioskop; Zeiss) with a computer-controlled motorized stepping stage. Tumour necrosis was evaluated, relative to the total tumour area, morphologically by using H\&E staining. Tumour hypoxic fraction and vascular density (structures per square millimeter) were determined relative to the viable tumour tissue (necrosis excluded).

\section{Click-it chemistry}

After 16 hours exposure to anoxia or normoxia, L-AHA $(50 \mu \mathrm{M})$ in methionine free medium was added and incubated for 60 minutes. Medium was changed and collected after 6-8 hours. Proteins were precipitated with chloroform/ methanol and resuspended in $0.5 \mathrm{M}$ tris- $\mathrm{HCl} 1 \% \mathrm{SDS}$. Click-it reaction was done according to the manufacturer's manual (Click-It ${ }^{\circledR}$ Invitrogen). Proteins were loaded on SDS-page gel and transferred to PVDF membrane, blocked with $2 \%$ casein. Newly synthesized proteins were visualized using streptavidin-HRP overnight at $4{ }^{\circ} \mathrm{C}$ in blocking buffer

\section{Cryo-Electron microscopy}

U87 derived hypoxic vesicles were isolated by differential ultracentrifugation as described previously and labeled with anti-GABARAPL1 (1:50). For visualization GABARAPL1 was labeled with anti-Rabbit IgG Gold antibody (anti-GABARAPL1, 11010-1$1 P$, protein tech group) (20nM). A thin aqueous film of the sample was formed on a Quantifoil R2/2 grid (Quantifoil GMbH, Jena BRD) by applying $2.5 \mu \mathrm{l}$ of sample and blotting away excess liquid. The grid was held by tweezers in the environmental chamber $\left(20^{\circ} \mathrm{C}\right.$ and more than $95 \%$ relative humidity) of the Vitrobot mark IV (a vitrification robot from FEI) (24). After blotting, the grid with the thin aqueous film was rapidly vitrified by plunging into ethane cooled to its melting point $\left(-180^{\circ} \mathrm{C}\right)$ by liquid nitrogen. The vitrified specimen was transferred to the cold stage (Gatan cryo-holder) of the transmission electron microscope (FEI-T12 Biotwin). The pictures were taken with a FEI-Eagle-4K-CCD camera.

\section{Statistics}

Data were analyzed with GraphPad Prism software. Multiple testing was done using a repeated-measure ANOVA with a Bonferroni post-hoc test. Student's $t$ test was used for single comparisons Statistical test used are described in each figure legend. All animal data are presented as mean \pm SEM. Data were considered statistically significant when $P$ was less than 0.05 . 


\section{Results}

Expression of fluorescently tagged GABARAPL1 (mCherry-GABARAPL1), followed by live cell imaging experiments revealed trafficking of vesicle-like structures towards the cells periphery, suggesting involvement in secretory mechanisms (supplementary video 1). To determine if GABARAPL1 was involved in secretion, we compared total protein secreted from control and GABARAPL1 knockdown cells exposed to normoxia $\left(20 \% \mathrm{O}_{2}\right.$ and hypoxia $\left(<0.02 \% \mathrm{O}_{2}\right)$. Click-it chemistry allowed us to specifically label de novo synthesized and secreted protein using L-azidohomoalaine (L-AHA) incorporation. L-AHA incorporated and secreted proteins were isolated from the culture medium, biotinylated and visualized by immunoblot analysis. We observed that during hypoxia total protein secretion is increased in HT29 and MCF7 cells. However, this effect is abolished in GABARAPL1 knockdown cells (fig 1A).

To identify which proteins are dependent on GABARAPL1 for secretion, we analysed secreted proteins through SILAC-based mass spectrometry (table 1). Proteins from control and knockdown cells were concentrated (10kDa cut-off) from the culture medium, labelled with light and heavy label, and analysed. Functional enrichment analysis of reduced secreted proteins ( $>10 \%$ difference) in GABARAPL1 deficient cells revealed enrichment of proteins involved in cell communication and cell proliferation and/or maintenance (fig 1B and table 1). Importantly, we observed a reduction of proteins enriched in EVs, including the tetraspanin, and EV-marker, CD81. Further analysis of these differentially secreted factors revealed that almost all proteins have been observed in, or have been associated with EVs (according to exocarta.org, table 1), suggesting a role in EV secretion.

\section{Table 1}

\begin{tabular}{|c|c|c|c|c|}
\hline \multicolumn{2}{|c|}{ Gene SymbolDescription } & \multirow{2}{*}{$\begin{array}{l}\text { Entrez } \\
\text { GenelD } \\
\\
60\end{array}$} & \multirow{2}{*}{$\begin{array}{l}\text { CONTROL/ } \\
\text { ShGABARAPL1 } \\
\\
-27 \%\end{array}$} & \multirow{2}{*}{$\begin{array}{l}\text { EV } \\
\text { associated } \\
\text { (exocarta. } \\
\text { org) } \\
\text { Yes }\end{array}$} \\
\hline ACTB & actin, beta & & & \\
\hline AMIGO2 & adhesion molecule with Ig-like domain 2 & 347902 & $-35 \%$ & Yes \\
\hline APLP2 & amyloid beta (A4) precursor-like protein 2 & 334 & $-49 \%$ & Yes \\
\hline ATP6AP1 & ATPase, $\mathrm{H}+$ transporting, lysosomal accessory protein 1 & 537 & $-29 \%$ & Yes \\
\hline CD81 & CD81 molecule & 975 & $-20 \%$ & Yes \\
\hline CLU & clusterin & 1191 & $-23 \%$ & Yes \\
\hline COL1A1 & collagen, type I, alpha 1 & 1277 & $-43 \%$ & Yes \\
\hline CTSB & cathepsin B & 1508 & $-11 \%$ & Yes \\
\hline CTSC & cathepsin C & 1075 & $-25 \%$ & Yes \\
\hline EPCAM & epithelial cell adhesion molecule & 4072 & $-15 \%$ & Yes \\
\hline FAT1 & FAT atypical cadherin 1 & 2195 & $-29 \%$ & Yes \\
\hline FRAS1 & Fraser extracellular matrix complex subunit 1 & 80144 & $-24 \%$ & - \\
\hline
\end{tabular}




\begin{tabular}{|c|c|c|c|c|}
\hline \multicolumn{2}{|c|}{ Gene SymbolDescription } & \multirow{2}{*}{$\begin{array}{l}\text { Entrez } \\
\text { GeneID } \\
\\
9245\end{array}$} & \multirow{2}{*}{$\begin{array}{l}\text { CONTROL/ } \\
\text { ShGABARAPL1 } \\
-23 \%\end{array}$} & \multirow{2}{*}{$\begin{array}{l}\text { EV } \\
\text { associated } \\
\text { (exocarta. } \\
\text { org) } \\
\text { Yes }\end{array}$} \\
\hline GCNT3 & glucosaminyl ( $\mathrm{N}$-acetyl) transferase 3 , mucin type & & & \\
\hline GDF15 & growth differentiation factor 15 & 9518 & $-37 \%$ & Yes \\
\hline GLA & galactosidase, alpha & 2717 & $-12 \%$ & Yes \\
\hline GOLM1 & golgi membrane protein 1 & 51280 & $-10 \%$ & Yes \\
\hline GPC1 & glypican 1 & 2817 & $-42 \%$ & Yes \\
\hline GSN & gelsolin & 2934 & $-34 \%$ & Yes \\
\hline HEXA & hexosaminidase A (alpha polypeptide) & 3073 & $-10 \%$ & Yes \\
\hline $\mathrm{HIST} 2 \mathrm{H} 2 \mathrm{BF}$ & histone cluster 2, H2bf & 440689 & $-47 \%$ & Yes \\
\hline HSP90AB1 & $\begin{array}{l}\text { heat shock protein 90kDa alpha (cytosolic), class B } \\
\text { member } 1\end{array}$ & 3326 & $-19 \%$ & Yes \\
\hline IGFBP4 & insulin-like growth factor binding protein 4 & 3487 & $-20 \%$ & Yes \\
\hline IGFBP6 & insulin-like growth factor binding protein 6 & 3489 & $-18 \%$ & Yes \\
\hline JAG1 & jagged 1 & 182 & $-17 \%$ & Yes \\
\hline LAMA5 & laminin, alpha 5 & 3911 & $-29 \%$ & Yes \\
\hline LAMC1 & laminin, gamma 1 (formerly LAMB2) & 3915 & $-26 \%$ & Yes \\
\hline LGMN & legumain & 5641 & $-13 \%$ & - \\
\hline LOXL4 & lysyl oxidase-like 4 & 84171 & $-13 \%$ & Yes \\
\hline MET & MET proto-oncogene, receptor tyrosine kinase & 4233 & $-24 \%$ & Yes \\
\hline NEO1 & neogenin 1 & 4756 & $-20 \%$ & Yes \\
\hline NUCB1 & nucleobindin 1 & 4924 & $-11 \%$ & Yes \\
\hline PLOD2 & procollagen-lysine, 2-oxoglutarate 5-dioxygenase 2 & 5352 & $-13 \%$ & Yes \\
\hline PTPRF & protein tyrosine phosphatase, receptor type, F & 5792 & $-51 \%$ & Yes \\
\hline S100A11 & S100 calcium binding protein A11 & 6282 & $-14 \%$ & Yes \\
\hline SDF4 & stromal cell derived factor 4 & 51150 & $-29 \%$ & Yes \\
\hline SEMA7A & $\begin{array}{l}\text { semaphorin 7A, GPI membrane anchor (John Milton } \\
\text { Hagen blood group) }\end{array}$ & 8482 & $-38 \%$ & - \\
\hline SERPINE2 & $\begin{array}{l}\text { serpin peptidase inhibitor, clade } \mathrm{E} \text { (nexin, plasminogen } \\
\text { activator inhibitor type 1), member } 2\end{array}$ & 5270 & $-53 \%$ & Yes \\
\hline SPINK1 & serine peptidase inhibitor, Kazal type 1 & 6690 & $-20 \%$ & Yes \\
\hline TFF1 & trefoil factor 1 & 7031 & $-22 \%$ & - \\
\hline THBS1 & thrombospondin 1 & 7057 & $-29 \%$ & Yes \\
\hline TINAGL1 & tubulointerstitial nephritis antigen-like 1 & 64129 & $-16 \%$ & Yes \\
\hline TSPAN1 & tetraspanin 1 & 10103 & $-28 \%$ & Yes \\
\hline TUBB4B & tubulin, beta 4B class IVb & 10383 & $-36 \%$ & Yes \\
\hline TWSG1 & twisted gastrulation BMP signaling modulator 1 & 57045 & $-33 \%$ & - \\
\hline
\end{tabular}


A

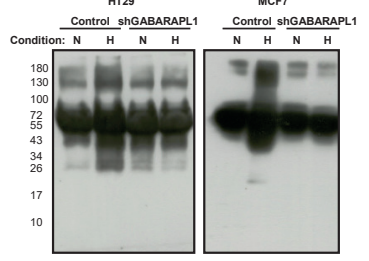

B

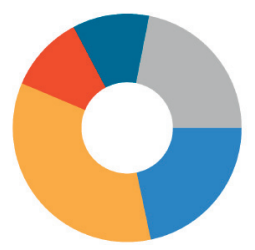

Cell growth and/ or maintenance $(P<0.001)$

Cell communication $(P<0.01)$

Protein metabolism ( $\mathrm{P}=0.21)$

Energy pathways $(P=0.35)$

Others
C
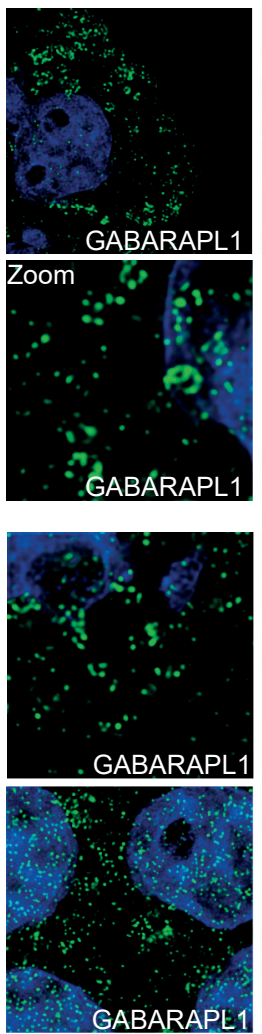
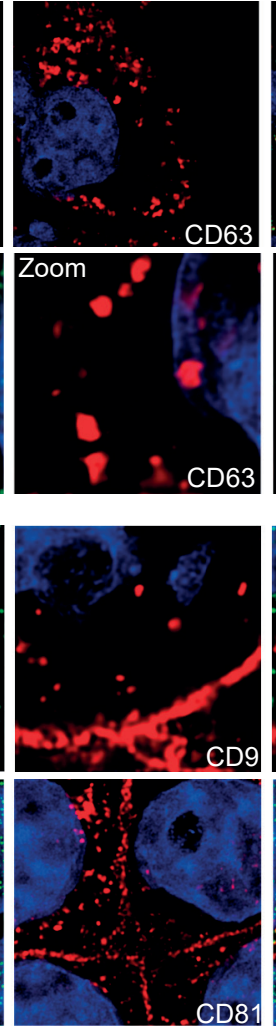
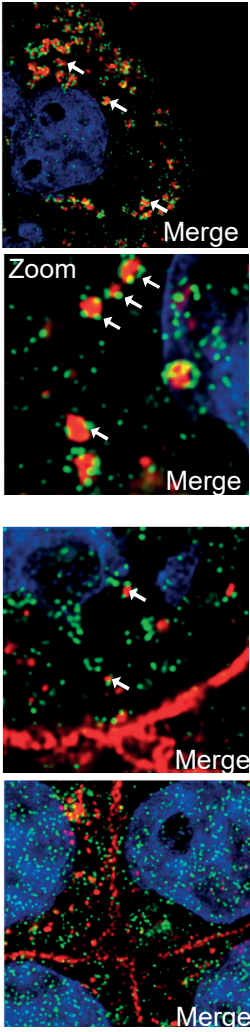

D



E

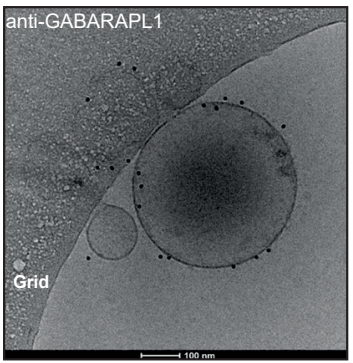

Figure 1 GABARAPL1 is associated with extracellular vesicles.

(A) Analysis of newly synthesized and secreted proteins probed by methionine pulse labeling followed by Click-it chemistry and strep-HRP visualization by immunoblot analysis indicates decreased protein secretion in HT29 and MCF7 cells during hypoxia. (B) Functional enrichment analysis (Fun Rich software) of GABARAPL1 dependent secreted proteins as determined by mass-spectrometry (table 1). (C) Confocal analysis of Immunofluorescent labeled GABARAPL1 (Green) with EV markers CD63 (red), CD81 (red) or CD9 (red) and Hoechst (blue) indicates intracellular colocalization. (D) Confocal microscopy of GABARAPL1 (green) and CD81 (red) indicate colocalization on vesicular extracellular structures. The plasma membrane is indicated by a white line.

(E) Cryo-electron micrograph of EVs after immuno gold-labeling with anti-GABARAPL1 indicates that GABARAPL1 expression is retained on the EV membrane after secretion. Size-bar is $100 \mu \mathrm{m}$. 
To further study the possible association between GABARAPL1 and EVs, GABARAPL1 and the EV markers CD81, CD9 and CD63 were fluorescently labeled after hypoxia exposure and cellular distribution was assessed by deconvolution confocal microscopy. In both HT29 (fig 1C) and U87 cells (suppl fig 1) intracellular endogenous GABARAPL1 seems to be co-expressed on the same structure with the tetraspanin CD63, and to a lesser extend CD9 and CD81. Typically located on the plasma membrane, CD81 and CD9 also show a cytoplasmic distribution in a vesicle-like pattern where they frequently are accompanied by GABARAPL1 positive structures (fig 1C).

Furthermore, immunostainings reveal both GABARAPL1 and CD81 positive EVs in the extracellular environment (fig 1D) but not with CD63 or CD9 (not shown), suggesting secretion of GABARAPL1. Immunogold-labeling of intact, unfixed EVs followed by cryo-EM visualization revealed that GABARAPL1 is, at least, present at the surface of secreted EVs (fig 1E). Intriguingly, although GABARAPL1 expression can be detected on small and larger vesicles, not all EVs express GABARAPL1 (data not shown), suggesting that GABARAPL1 marks a subset of EVs.

\section{GABARAPL1 is required for EV secretion.}

Although highly enriched on EVs after secretion, intracellular CD63 indicates the presence of multivesicular bodies/late endosomes. As we observed that, during hypoxia, GABARAPL1 intracellularly colocalizes with CD63 and is present on EVs after secretion, we questioned if GABARAPL1 has a functional role in EV secretion. To test this, EVs were isolated from HT29 and U87 cell culture supernatant by differential ultracentrifugation and further purified by density gradient flotation. This technique allows to isolated EVs based on their density rather than size (20). Gradients were divided in 12 fractions and EV composition was characterized by analyzing CD81, CD9 and CD63 expression by western blot. GABARAPL1 knockdown resulted in changes in EV marker secretion in both HT29 and U87 cells (fig 2A). Secretion of CD9 and CD81 expressing EVs is strongly reduced, whereas secretion of CD63 is less dependent on GABARAPL1 expression. Interestingly, in HT29 cells CD63 secretion is also reduced, suggesting a role for GABARAPL1 in overall EV secretion. In U87, GABARAPL1 knockdown resulted in increased CD63 secretion. These results were validated using independent shRNA's targeting GABARAPL1 (Suppl fig 2A). In addition, CD81 and CD63, but not CD9 (suppl. fig 2B) secretion is decreased after GABARAPL1 knockdown in the breast cancer cell line, MCF7 (suppl fig 2B). These data suggest that every cell line has its own repertoire in the total EV populations with sub-populations that are produced and secreted by independent mechanisms. The role of GABARAPL1 and which of the independent mechanisms depends on GABARAPL1-expression remains to be elucidated. Alternatively, given the biological importance of intercellular communication through EVs, compensatory mechanisms may have been lost or gained in the process of tumorigenesis and may explain the cell-dependent effects on EV-marker secretion. 
A
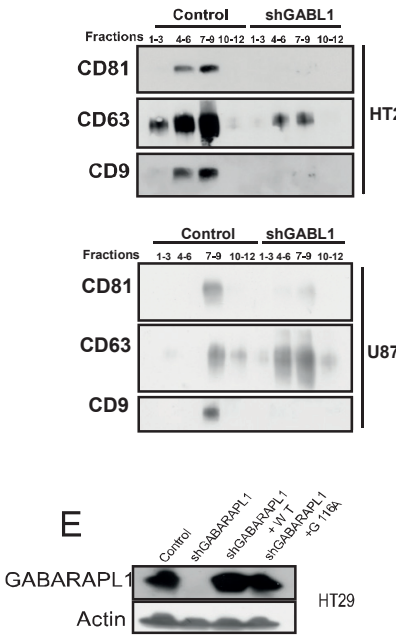

$\mathrm{F}$

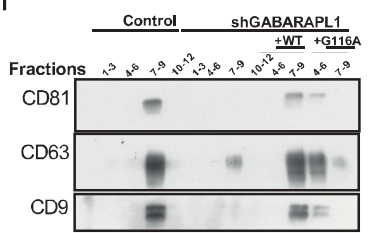

B

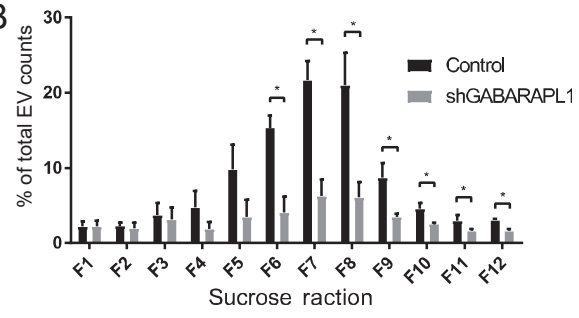

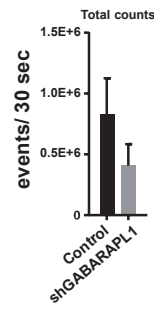

C

$\mathrm{D}$
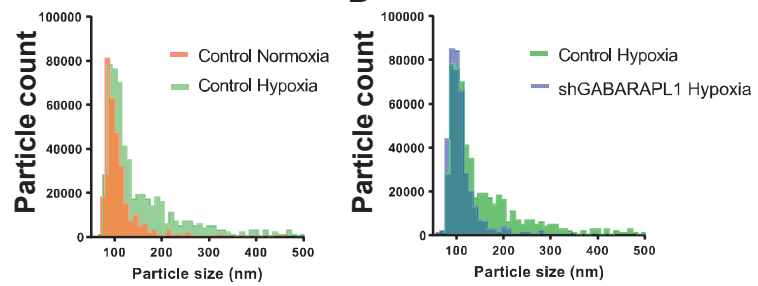

G

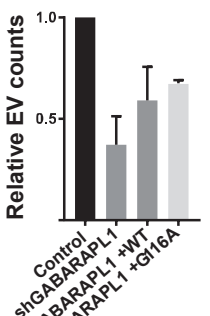

$\mathrm{H}$

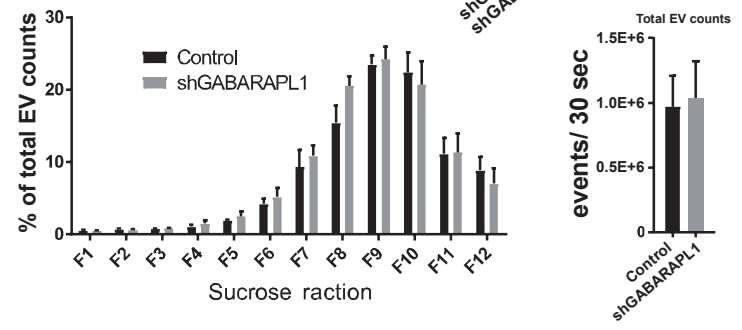

Figure 2 GABARAPL1 is required for EV secretion.

(A) Secreted EV marker evaluation after EV isolation by differential ultracentrifugation followed by immunoblot analysis reveals reduced marker abundance in secreted EVs of HT29 and U87 cells. Sucrose density fractions are pooled per 3. (B) High resolution flow cytometry of sucrose fractions after centrifugation indicates reduced EV secretion in GABARAPL1 deficient cells. (C) Size distribution analysis of secreted EVs by resistive pulse sensing shows that a larger subpopulation is secreted during hypoxia (green) which is (D) GABARAPL1 dependent (red). (E) Immunoblot analysis of GABARAPL1 protein expression of HT29 rescue model. (F) Reexpression of wild-type (WT) or mutant (G116A) GABARAPL1 restores the marker composition of secreted EVs as assessed by immunoblot analysis or (G) high resolution flow cytometry after sucrose density purification. Plotted are total counts of fraction 4-12. (H) Distribution of EV counts as assessed by high-resolution flow cytometry of U87 cells. 
To determine whether only the composition of EVs has changed, or also the number of secreted EVs, EV secretion was quantified using high-resolution flow cytometry (21). EVs were isolated from the culture medium of hypoxic cells using differential ultracentrifugation, fluorescently labeled with PKH67 and floated to their buoyant density using density gradient floatation. We observed that the number of secreted EVs is decreased in HT29 after GABARAPL1 knockdown (fig 2B). To validate these results and to determine the accurate size of the secreted EVs we used Resistive Pulse Sensing. We observed that during hypoxia HT29 cells secrete a subpopulation of an additional larger size (>150nm) during hypoxia (fig 2C) which is dependent on GABARAPL1 expression (fig 2D). Rescue of GABARAPL1 expression with a non-targetable coding sequence (fig 2E) resulted in normalization of EV-marker secretion in HT29 cells (fig 2F) and increased number of EV secretion as determined by high resolution flow cytometry (fig 2G).

All LC3/GABARAP family proteins can be post translationally modified through Cterminal lipidation with phosphatidylethanolamine (PE) to allow membrane association $(25,26)$. Interestingly, rescuing GABARAPL1 expression using a PE-conjugation defective GABARAPL1 mutant (GABARAPL1 G116A) also partially rescued EV secretion (fig 2E and $F$ ), indicating that the association of GABARAPL1 with secretory vesicles is not PE dependent and suggests another feature to interact with lipid bilayers.

Interestingly, although GABARAPL1 knockdown results in decreased CD81 and CD9 expression on EVs secreted by U87 cells, we did not observe a decrease in the number of secreted EVs in both independent shRNA's (fig $2 \mathrm{H}$ and suppl fig 2D). The difference between the cell lines, the effect on EV marker composition and counts of secreted EVs suggest that compensatory mechanisms may be utilized or that different cell lines use different secretory mechanisms for secretion of EVs (14).

\section{GABARAPL1 is required for hypoxic cells to stimulate angiogenesis}

To alleviate tumour hypoxia, cells respond to low oxygenation by releasing growth factors that stimulate angiogenesis (4). Mass spectrometry analysis already revealed several proand anti-angiogenic growth factors dependent on GABARAPL1 expression for secretion (table 1). EVs derived from hypoxic cells have previously been demonstrated to stimulate endothelial tube formation in vitro $(19,27)$. To test whether the changes in EV secretion and angiogenic factor secretion after GABARAPL1 depletion altered the angiogenic potential of hypoxic cells, endothelial cells were exposed to EVs derived from hypoxic control and GABARAPL1 knockdown cells and tube formation was assessed. EVs derived from GABARAPL1 knockdown cells display reduced angiogenic potential (fig 3A).

To determine which factors are involved in GABARAPL1 mediated angiogenesis, the expression levels of 55 angiogenesis associated proteins were determined in cell culture supernatant using antibody arrays (fig 3B). Quantification of factors above detection limit reveals that secreted levels of several factors are altered in GABARAPL1 deficient 
U87 (fig 3B), HT29 (fig 3C) and MCF7 cells (suppl fig 3A). These effects are most pronounced during hypoxia.

GABARAPL1 expression at the surface of secreted EVs (fig 1E) allows targeting of the vesicles. To evaluate if EV membrane-expressed GABARAPL1 is required for EV-mediated effects, GABARAPL1 was targeted with antibodies. HUVEC stimulation with EVs in the presence of GABARAPL1 targeting antibodies results in abrogation of endothelial tube formation (fig 3D). Endothelial tube formation by direct addition of soluble growth factors (VEGF, FGF2, IGF, EGF) could not be prevented by GABARAPL1-targeting antibodies (fig $3 E$ ), indicating an EV mediated effect. Whether this effect is mediated by steric hindrance or prevention of GABARAPL1-target interaction remains to be elucidated.

A
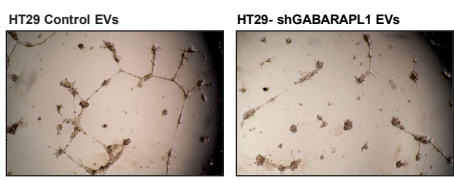

$\mathrm{B}$

U87 normoxia

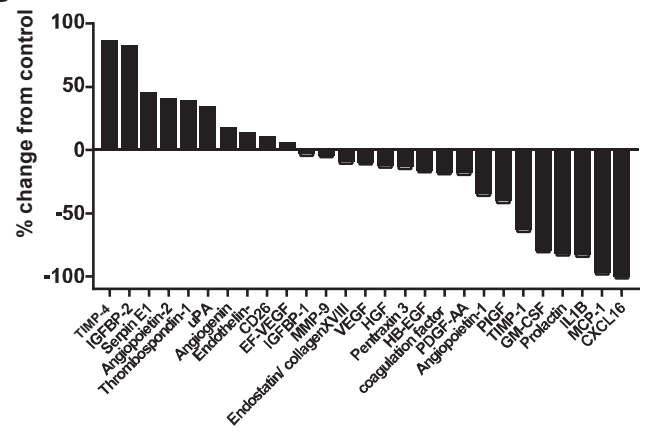

U87 hypoxia
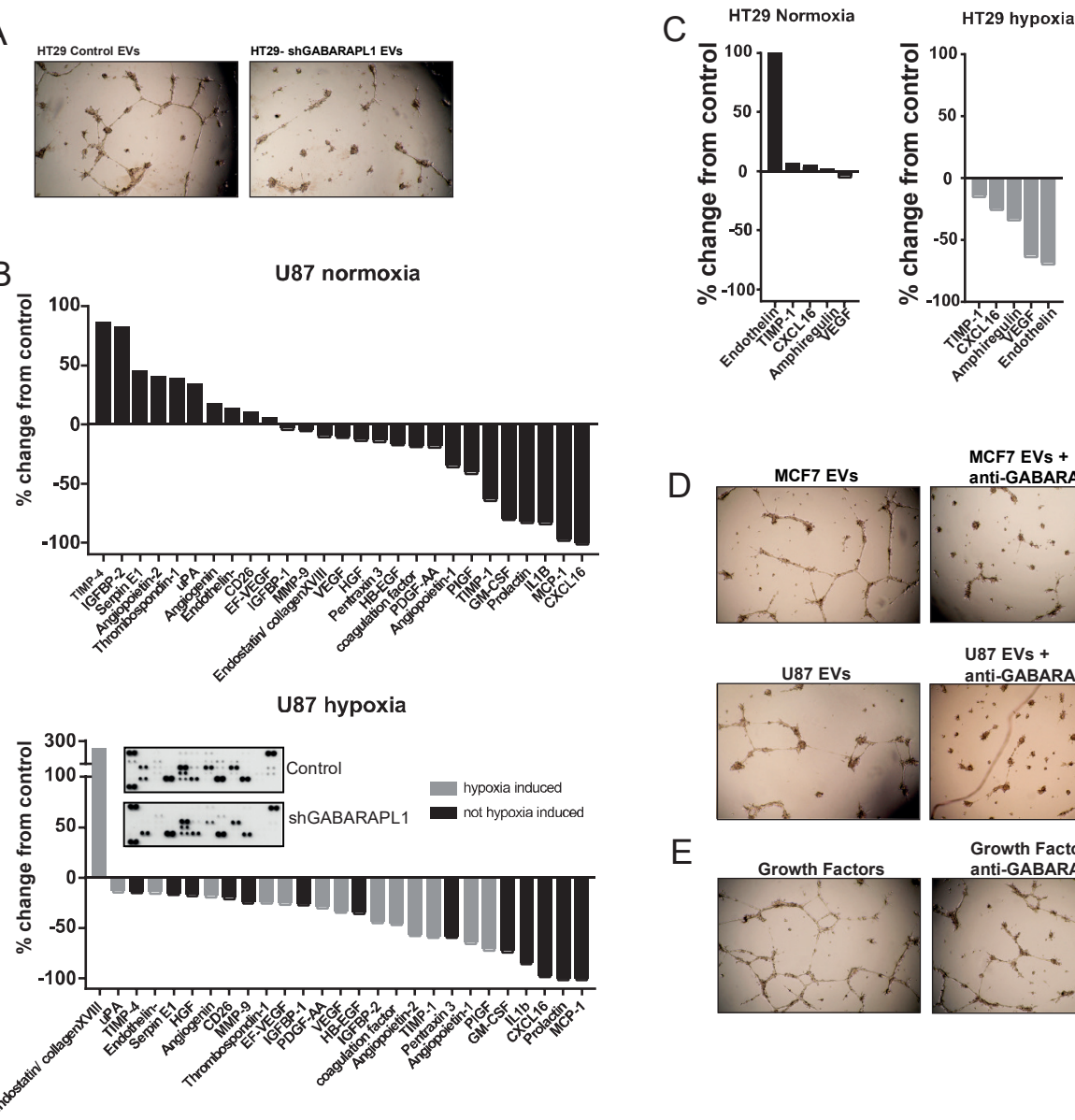

$\mathrm{D}$
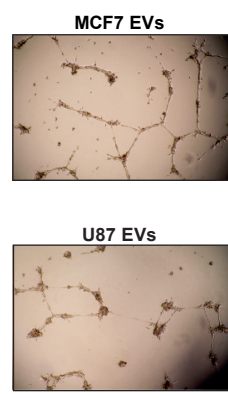

$E$

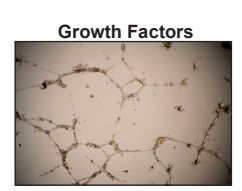

MCF7 EVs +

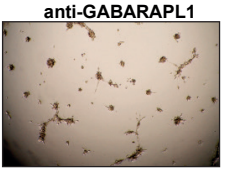

U87 EVs +

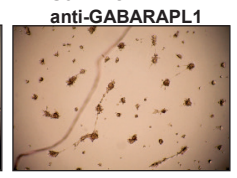

Growth Factors + anti-GABARAPL1

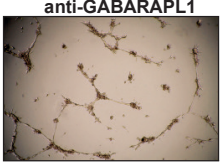

Figure 3 GABARAPL1 deficient cells display decreased angiogenic potential.

(A) Hypoxic HT29 derived EVs induce tube formation in HUVEC cells after stimulation with EVs isolated from control but not GABARAPL1 deficient cells. (B) Quantification of antibody-arrays of U87 conditioned medium of control and GABARAPL1 knockdown cells. Plotted is the fold change from control. Insert is a representative example of antibody array membranes. (C) Quantification of antibody-array of HT29 control and 
shGABARAPL1 cells. (D) Tube formation is blocked by using GABARAPL1 antibodies. HUVEC were stimulated with hypoxic EVs from U87 and MCF7 wild-type cells. Tube-formation is blocked when EVs are incubated with GABARAPL1 antibodies. (E) Antibodies with soluble growth factors (VEGF, FGF2, IGF, EGF) cannot prevent tube-formation in HUVEC cells.

\section{GABARAPL1 supports tumour progression through enhancement of angiogenesis}

Our in vitro experiments indicate that GABARAPL1 is required for the hypoxia-induced secretion of EVs that are required to elicit pro-angiogenic effects. To test this in a tumour setting, U87 cells were subcutaneously grown in the flanks of female nu/nu NMRI mice. Cells were engineered to express shRNA under a doxycycline-inducible promoter. Doxycycline was administered through the drinking water. GABARAPL1 deficiency resulted in delayed tumour growth (fig $4 \mathrm{~A}$ and $\mathrm{B}$ ) and was associated with reduced vessel formation (31\% difference, $\mathrm{p}=0.005$ (fig 4C), as assessed by $9 \mathrm{F1}$ immunohistochemistry. Despite lower vessel density no differences in tumour hypoxia, as assessed by pimonidazole immunohistochemistry, or differences in vessel perfusion were observed (suppl fig 4A).

Comparable results were obtained with HT29 xenografts. We observed that GABARAPL1 knockdown reduced tumour growth (fig 4D) as illustrated by an increase in tumour doubling times from 11 to 15 days (36\%) (fig 4E). Pimonidazole and 9F1 immunohistochemical analysis (fig 4F) disclosed that also in this model the vessel density was reduced after GABARAPL1 knockdown (fig $4 \mathrm{H}$ ). The levels of hypoxia of the viable tissue did not differ from controls (fig 4I). Interestingly, morphology assessment using H\&E staining indicated that GABARAPL1 deficient tumours display increased tumour necrosis (fig $4 \mathrm{G}, \mathrm{J}$ ). Vessel perfusion remained unchanged (suppl fig 4B).

Next we questioned if GABARAPL1 targeting could be exploited therapeutically. The doxycycline inducible promoter allows establishment of tumours with intact GABARAPL1 expression, which can then be transiently blocked by addition of doxycycline to the drinking water. No significant difference in growth rates between, as wildtype established, control and GABARAPL1 knockdown tumours was observed (fig 4K, L, dotted lines) indicating that already existing vessel networks are not affected. To determine if GABARAPL1 targeting could potentially be used as an adjuvant therapy we allowed xenografts to reach a volume of $150 \mathrm{~mm}^{3}$, irradiated the tumours with a single, tumour specific dose of 10Gy followed by GABARAPL1 knockdown through doxycycline administration. We observed that targeting GABARAPL1 after irradiation significantly reduced regrowth of the tumours (fig $4 \mathrm{~K}, \mathrm{~L}$ ) which was again associated with reduced vessel density (fig $5 \mathrm{M}$ ). This finding is potentially interesting for incurable tumours or tumours treated with palliative intent. Although GABARAPL1 knockdown was induced after irradiation, irradiation-induced cell death may take several days. To exclude effects on intrinsic radiosensitivity after GABARAPL1 depletion, clonogenic survival was determined after irradiation. No effect of GABARAPL1 knockdown was observed on intrinsic radiosentivity of cells (suppl fig $4 \mathrm{C}$ ), suggesting that the observed growth delay after irradiation is caused by reduced tumour-supportive angiogenesis. 
A

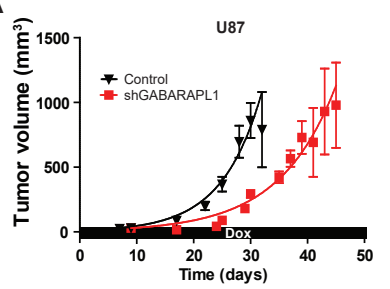

D

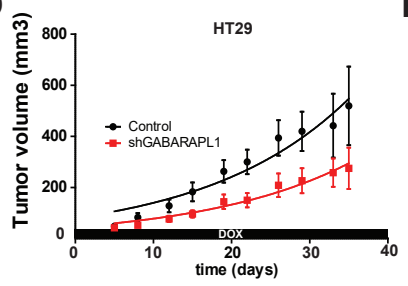

H

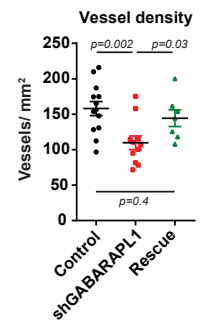

K

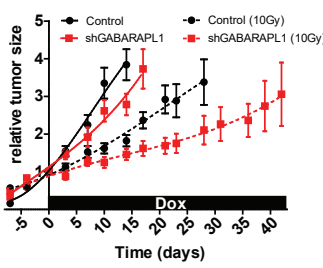

0
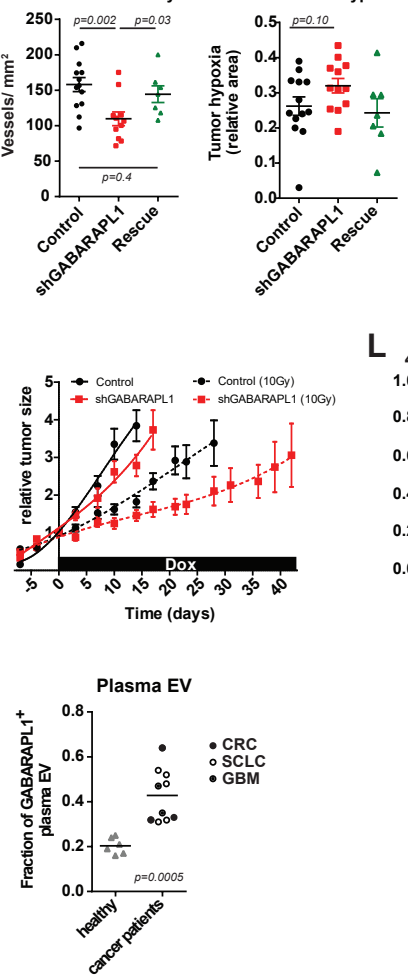

L
B

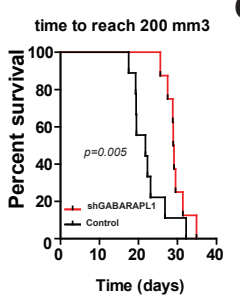

E

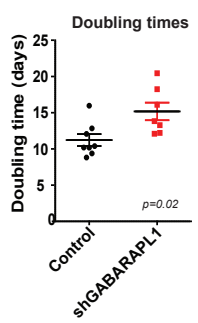

F
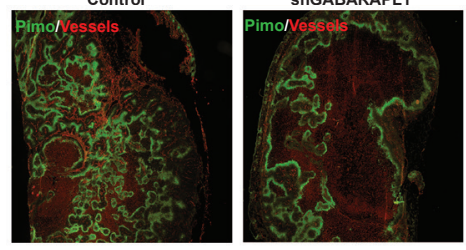

G
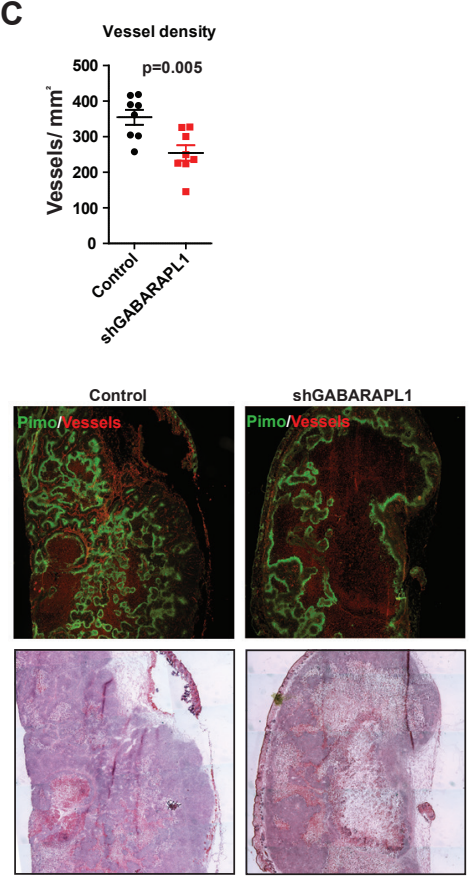
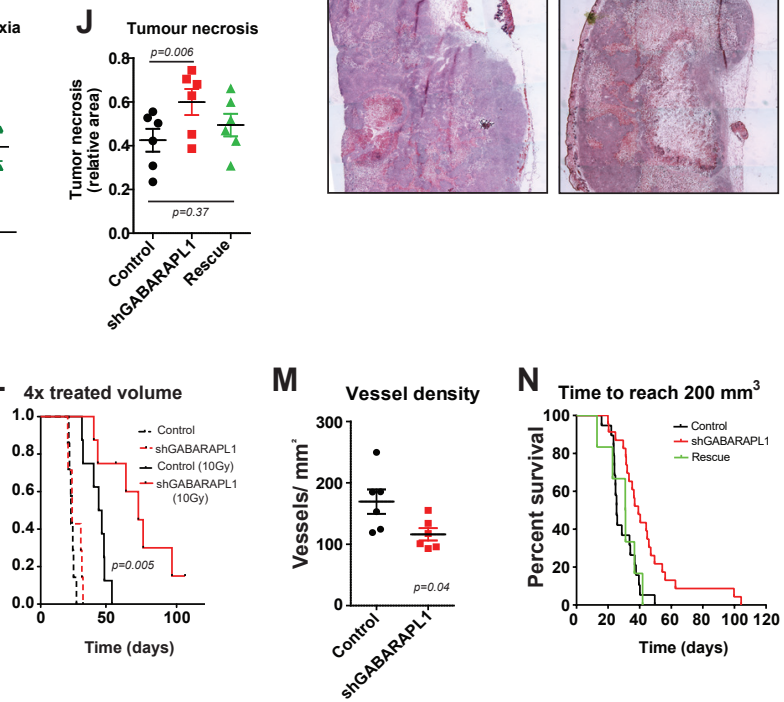

Figure 4 GABARAPL1 contributes to tumour progression by enhancing angiogenesis.

$(\mathbf{A}, \mathbf{B})$ Doxycycline (dox)-inducible U87 GABARAPL1 knockdown $(n=8)$ and control cell lines $(n=9)$ were injected subcutaneously in the flanks of nMRI nu/nu mice. Dox was given through the drinking water to induce knockdown. GABARAPL1 knockdown results in delayed tumour growth of subcutaneous U87 xenografts. (Mean +/SEM, survival curves were analyzed using Wilcoxon log rank, $p=0.05$ ). (C) GABARAPL1 knockdown is associated 
with decreased vessel density ( $p=0.005$, Student's t-test unpaired, 2-tailed, mean +/- SEM). (D,E) GABARAPL1 knockdown results in decreased tumour growth of subcutaneous HT29 xenografts and increased doubling times $(n=8)$ ( $p=0.02$ Student's t-test unpaired, 2-tailed, mean +/- SEM). (F) cross-section of control and knockdown tumours with matching (G) H\&E stainings, assessed for hypoxia (pimonidazole, green) and vessels (9F1, red). (H) GABARAPL1 knockdown results in decreased vessel density (control $n=6$, shGABARAPL1 $n=4$, $p=0.18$ Student's t-test unpaired, 2-tailed, mean +/- SEM). (I) Quantification of hypoxic areas of the viable tissue from control (black) and GABARAPL1 deficient tumours (red) ( $p=n . s, n=6$. Student's t-test unpaired, 2tailed, mean +/- SEM). (J) Quantification of necrotic fraction from control (black) and GABARAPL1 deficient tumours (red) ( $p=0.006, n=6$. Student's t-test unpaired, 2-tailed, mean +/- SEM) and GABARAPL1 deficient tumours after contralateral implantation of control tumour (green) ( $p=n . s, n=6$. Student's t-test unpaired, 2tailed, mean +/- SEM). (K) Knockdown of GABARAPL1 does not affect tumour growth of established HT29 tumours. A single dose of 10 Gy delays tumour regrowth of GABARAPL1 deficient tumours compared to control tumours ( $n=8$ in all groups). (L) Survival-curve of tumours reaching $4 x$ treated volume. Curve comparison between Control 10Gy $(-)$ ) and shGABARAPL1 10Gy $(-)$ is done by Gehan-Breslow-Willcoxon test, $p=0.005$. (M) GABARAPL1 knockdown is associated with decreased vessel density after irradiation (control $n=6$, shGABARAPL1 $n=6, p=0.04$ Student's t-test unpaired, 2-tailed, mean +/- SEM). (N) Survival curves of tumours reaching $200 \mathrm{~mm}^{3}$. Growth of Rescue shGABARAPL1 $(-, n=6)$ is partially rescued. GABARAPL1 knockdown tumours $(-, n=15)$, Control tumours $(-n=11)$. Curve comparison was done by GehanBreslow-Willcoxon test. Control VS Rescue $p=0.4$, shGABARAPL1 VS Rescue $p=0.16$ ). (0) Vessel density is restored in Rescue tumours. Control VS Rescue $p=0.4$. shGABARAPL1 vs Rescue $p=0.04$, Student's t-test unpaired, 2-tailed, mean +/- SEM)

Finally, we and others have demonstrated that GABARAPL1 is involved in anterograde trafficking of several receptors, including EGFR, which might influence tumour cell proliferation (9). To determine to what extent the observed effects of GABARAPL1 deficiency on tumour growth and the tumour microenvironment are EV-mediated or are a consequence of cell intrinsic changes, animals were implanted with 2 control (control), 2 GABARAPL1 knockdown (shGABARAPL1) tumours, or 1 GABARAPL1 knockdown combined with 1 control tumour on the contralateral flank (rescue). Again, GABARAPL1 deficiency results in delayed tumour growth of GABARAPL1-deficient tumours compared to control tumours (fig $4 \mathrm{~N}$ ). Importantly, the delayed tumour growth of shGABARAPL1 tumours was partially rescued if the same animal was implanted with a control tumour (fig $4 \mathrm{~N}$ ). In addition, the reduced vessel density (fig $4 \mathrm{H}$ ) and increased tumour necrosis (fig 4J) of GABARAPL1-deficient tumours was also resolved. These results support our observation that GABARAPL1 is important for the development and secretion of hypoxia-induced EVs that are required to induce angiogenesis.

These results indicate systemic delivery of GABARAPL1 $1^{+} \mathrm{EV}$ that mediate distant communication. To evaluate if we were able to detect GABARAPL1 ${ }^{+}$EVs in blood, we analysed the EV content of 10 cancer patients (2 glioblastoma multiforme (GBM), 5 small cell lung cancer (SCLC), 2 colorectal cancer (CRC)) and 6 healthy controls. In healthy controls, $20 \%$ of circulating EVs appear to be GABARAPL1 positive in contrast to $43 \%$ in cancer patients (fig $4 \mathrm{O}$ and suppl fig 4D). These data support our in vivo experiments were contralateral growing tumours are influenced by circulating GABARAPL1+ EVs. 


\section{Discussion}

\section{GABARAPL1 is not essential for autophagy}

The work described here shows a tumour-promoting role for GABARAPL1. As a member of the LC3/GABARAP protein family, it is generally assumed that GABARAPL1 is implicated in the general execution of autophagy. Similar to LC3B, we and others observed that GABARAPL1 is present in the cell in a vesicle like pattern (fig $1 \mathrm{C}$ and fig 5) (28). We unexpectedly observed that GABARAPL1 is not required for the general execution of autophagy during hypoxia in various tumour cell types, but is required for the secretion of EVs and growth factors during hypoxia and contributes to tumour growth.

It is suggested that GABARAPL1 plays a role in the later stages of autophagosome formation (29) and acts as autophagy receptor for selective autophagy (10). Despite several studies that show promising links between GABARAPL1 and autophagy associated processes, its exact functional role has, by the best of our knowledge, not been established so far. Co-immunostaining revealed that GABARAPL1 only partially colocalizes with LC3B in cells, suggesting different functions or involvement in differential aspects of autophagy execution (fig 5A and B). The golden standard used to determine the level of autophagy is by determining the level of LC3B turnover with and without the addition of an autophagy inhibitor (chloroquine) (the autophagic flux) (30). We observed that silencing GABARAPL1 does not inhibit autophagic flux, and in some cases even seems to induce autophagy in 2 independent hairpins in HT29 and U87 cells (fig 5D). Moreover, GABARAPL1 knockdown did not affect survival of cells exposed to hypoxia, in contrast to autophagy inhibition by pharmacological inhibitors or knockdown models (fig 5E) $(6,31)$. That GABARAPL1 knockdown increases autophagic flux conflicts with a previous report (32) that shows a reduced autophagic flux in MDA-MB-436 cells and activating procancer responses like increased proliferation and invasion upon GABARAPL1 silencing. Typically, autophagy inhibition decreases pro-cancer responses $(33,34)$, suggesting additional roles for GABARAPL1 rather than autophagy. These studies show that the role of GABARAPL1 in autophagy is still unclear and observed effects are likely to be cell type and stimulus dependent.

The autophagy machinery is devoted to form intracellular vesicles, or autophagosomes, for degradation, but also facilitates forms of unconventional secretion (35). Potentially, the GABARAPL1+ ${ }^{+}$vesicles described in this manuscript reflect the vesicles that are required for unconventional secretion. 
A
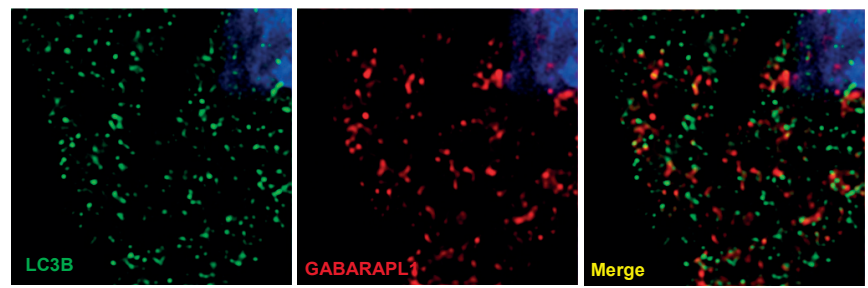

B
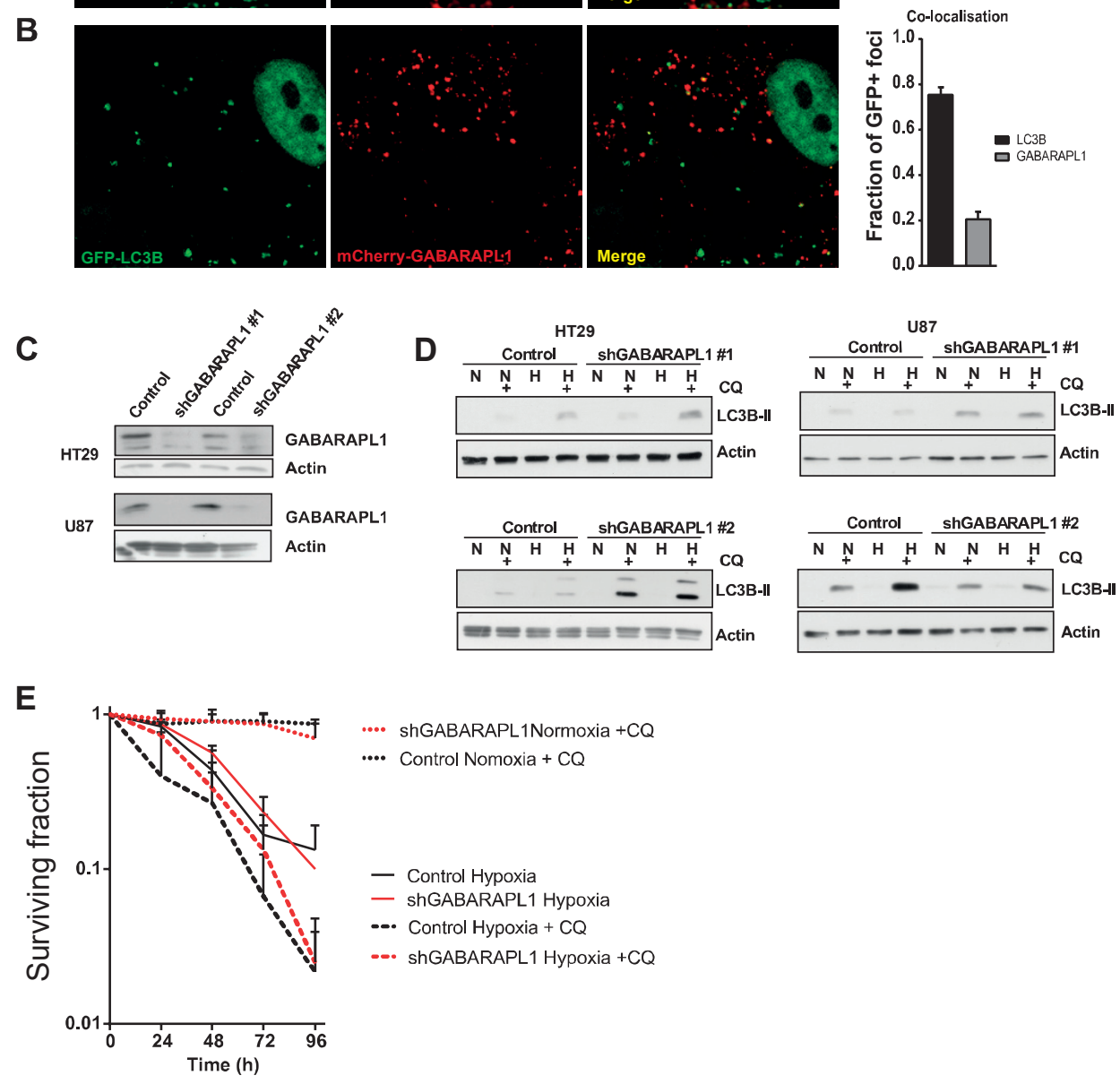

Figure 5 GABARAPL1 is not required for autophagy.

(A) Expression of fluorescently labeled endogenous GABARAPL1 (red) and LC3B (green) show partial colocalization in HT29 cells. Nucleus is depicted in blue. (B) Quantification of co-localization of GFP-LC3B and mCherry-GABARAPL1. Quantitative analysis is performed on the fraction GFP-LC3B positive foci co-localizing with mCherry positive foci. GFP-LC3B/mCherry-LC3B colocalization was used as control (black bar). (C) Immunoblot analysis of HT29 and U87 with control or inducible shRNA targeting GABARAPL1. (D) Immunoblot analysis indicates increased autophagic flux after GABARAPL1 knockdown in HT29 and U87 cells in 2 independent hairpins. $\mathrm{N}=$ Normoxia, $\mathrm{H}=$ Hypoxia $\left(<0.02 \% \mathrm{O}_{2}\right) \mathrm{CQ}=$ Chloroquine (Representative example of 6 experiments) (E) GABARAPL1 is not required for survival and is still sensitive to chloroquine during hypoxia $\left(<0.02 \% \mathrm{O}_{2}\right)$, as assessed in HT29 cells by clonogenic survival assays. $n=3+/-$ SEM. 


\section{GABARAPL1 is required for EV secretion}

In this manuscript we show that GABARAPL1 is required for secretion of EVs during hypoxia. Interestingly, silencing GABARAPL1 in U87 cells reveals decreased release of several EV markers by immunoblot, but not on total secreted EV numbers. Since not all markers (CD63) are affected by silencing GABARAPL1 this suggest that GABARAPL1 might be responsible for the secretion of a subset of EVs, or that other compensatory mechanisms are activated. It is generally accepted that the total EV pool released by a cell consists of subpopulations with different marker compositions. For example, Bobrie et al showed that silencing Rab27a in HELACIITA cells results in decreased secretion of classical EV markers (CD63, TSG101 and Alix) but did not affect others like CD9 (14). In addition, Groot Kormelink et al demonstrated that mast cell derived EV contain $\mathrm{CD}^{2} 3^{+}$a EVs subset with a smaller size and different phospholipid composition when compared to CD9 positive EVs (15). Collectively, these findings suggest that CD9 positive EVs origin from other cellular compartments and that GABARAPL1 is required for EV biogenesis rather than general EV secretion. That GABARAPL1 is required for secretion of a hypoxic subpopulation is supported by resistive pulse sensing (qNANO) measurements. We observed that a GABARAPL1-dependent larger subset (>150 nm) is secreted during hypoxic conditions. In line, Ramteke et al.(36) (37) also showed changes in EV size during hypoxia in PC3 cells, although they do not elaborate on hypoxic subsets.

Functional assays show that hypoxia-induced EVs enhance tube formation in HUVEC cells, an effect that can be abrogated by silencing or targeting GABARAPL1. In line with these results, we found that in two xenografted tumour types GABARAPL1 deficient tumours display decreased vessel density. Surprisingly, in contrast to HT29 xenografts, GABARAPL1 deficient U87 tumours did not grow slower compared to control tumours, despite a reduced vessel density. These effects may be related to the already very high vascular density of glioblastoma tumours.

Furthermore, as we showed previously, GABARAPL1 is also required for hypoxia induced membrane expression of EGFR and other hypoxia associated factors (9). Thus, inhibition of GABARAPL1 could potentially alter tumour growth by affecting intrinsic factors e.g. downstream signalling of the EGFR, leading to a decreased cell proliferation resulting in decreased tumour growth. Although in vitro experiments suggest that the observed effects on tumour development are GABARAPL1 ${ }^{+}$EV related, it is challenging to discriminate if the observed effects in tumour growth in vivo are solely due to GABARAPL1 ${ }^{+}$EV or also caused by cell intrinsic changes. To test this, GABARAPL1 knockdown tumours should be treated with control EVs. However, administration of EVs in vivo has several disadvantages; not in the least that sample collection and handling might change EVs behaviour of EVs. To overcome this issue, control tumours were implanted at the contralateral flank in the same animal. Zomer et al. showed that distant tumours release and exchange functional biomolecules, mediated by EVs (38). Indeed, we found that the decreased growth of GABARAPL1 knockdown tumours could be res- 
cued partially by circulating factors secreted by control tumours in a GABARAPL1 dependent manner.

GABARAPL1 ${ }^{+}$EVs are detectable and increased in the blood of cancer patients as compared to healthy controls, as we showed by confocal microscopy analysis. However, confocal microscopy is a suboptimal method for the analysis and quantification of EVs, but by our knowledge there is yet no other method available for the quantification of labelled EVs. The analysis of immobilized EVs by confocal microscopy might introduce a bias towards larger EVs and will therefore not be representative for the whole EV population. Nevertheless, GABARAPL1+ EVs are detectable in blood, which opens a window of opportunity for the development of biomarkers or therapeutics. Hypoxic cell-derived GABARAPL1+ EVs contribute to tumour growth. As GABARAPL1 was found to be present on the outer membrane of secreted EVs, this might be an interesting therapeutic target. As a proof of principle, we showed that the angiogenic function of GABARAPL1 ${ }^{+}$EVs could be blocked in vitro by the use of antibodies directed against GABARAPL1. Extrapolated to cancer patients this could mean that GABARAPL1 blocking antibodies could inhibit angiogenesis or neovascularisation and thereby inhibit tumour growth and increase susceptibility to therapeutic interventions. This was supported by our observation that inhibition of GABARAPL1 following irradiation contributed to delayed tumour regrowth.

In conclusion, we show for the first time that GABARAPL1 is important for tumour development, through secretion of a specific subset of pro-angiogenic EVs, marked by GABARAPL1 expression. Our data show that these EVs are detectable, targetable and are interesting to pursue as therapeutic target.

\section{Author contributions}

T.G.K. developed the hypothesis, designed the experimental approach, analyzed and interpreted the data, performed experiments, wrote the manuscript. S.F.L. Performed High-Resolution Flow cytometry experiments. M.B.E.S did co-localization experiments, processed samples. H.J.M.P and K.G.S performed western blots, DNA-cloning, processed samples, analyzed data and performed fluorescent stainings. J.B performed immunohistological staining's. H.D performed cryo-EM experiments. M.I.Z contributed to writing the manuscript. K.B. and J.A.D performed and analyzed SILAC mass-spectrometry experiments. M.V Contributed to writing the manuscript. M.W. contributed to hypothesis generation and interpretation of the data. K.M.A.R coordinated the project, developed the hypothesis, interpretation of the data, and contributed to writing the manuscript. 


\section{References}

1. Nordsmark M, Bentzen SM, Rudat V, Brizel D, Lartigau E, Stadler P, et al. Prognostic value of tumor oxygenation in 397 head and neck tumors after primary radiation therapy. An international multi-center study. Radiother Oncol 2005;77:18-24

2. Brizel DM, Sibley GS, Prosnitz LR, Scher RL, Dewhirst MW. Tumor hypoxia adversely affects the prognosis of carcinoma of the head and neck. Int J Radiat Oncol Biol Phys 1997;38:285-9

3. Hockel M, Schlenger K, Aral B, Mitze M, Schaffer U, Vaupel P. Association between tumor hypoxia and malignant progression in advanced cancer of the uterine cervix. Cancer Res 1996;56:4509-15

4. Harris AL. Hypoxia--a key regulatory factor in tumour growth. Nat Rev Cancer 2002;2:38-47

5. Wouters BG, Koritzinsky M. Hypoxia signalling through mTOR and the unfolded protein response in cancer. Nat Rev Cancer 2008;8:851-64

6. Rouschop KM, van den Beucken T, Dubois L, Niessen H, Bussink J, Savelkouls K, et al. The unfolded protein response protects human tumor cells during hypoxia through regulation of the autophagy genes MAP1LC3B and ATG5. J Clin Invest 2010;120:127-41

7. Dewhirst MW, Cao Y, Moeller B. Cycling hypoxia and free radicals regulate angiogenesis and radiotherapy response. Nat Rev Cancer 2008;8:425-37

8. Hanahan D, Folkman J. Patterns and emerging mechanisms of the angiogenic switch during tumorigenesis. Cell 1996;86:353-64

9. Keulers TG, Schaaf MB, Peeters HJ, Savelkouls KG, Vooijs MA, Bussink J, et al. GABARAPL1 is required for increased EGFR membrane expression during hypoxia. Radiother Oncol 2015;116:417-22

10. Schaaf MB, Keulers TG, Vooijs MA, Rouschop KM. LC3/GABARAP family proteins: autophagy-(un)related functions. Faseb J 2016;30:3961-78

11. Andreu Z, Yanez-Mo M. Tetraspanins in extracellular vesicle formation and function. Front Immunol 2014;5:442

12. Lotvall J, Hill AF, Hochberg F, Buzas El, Di Vizio D, Gardiner C, et al. Minimal experimental requirements for definition of extracellular vesicles and their functions: a position statement from the International Society for Extracellular Vesicles. J Extracell Vesicles 2014;3:26913

13. Van Deun J, Mestdagh P, Agostinis P, Akay O, Anand S, Anckaert J, et al. EV-TRACK: transparent reporting and centralizing knowledge in extracellular vesicle research. Nat Methods 2017;14:228-32

14. Bobrie A, Colombo M, Krumeich S, Raposo G, Thery C. Diverse subpopulations of vesicles secreted by different intracellular mechanisms are present in exosome preparations obtained by differential ultracentrifugation. J Extracell Vesicles 2012;1

15. Groot Kormelink T, Arkesteijn GJ, van de Lest CH, Geerts WJ, Goerdayal SS, Altelaar MA, et al. Mast Cell Degranulation Is Accompanied by the Release of a Selective Subset of Extracellular Vesicles That Contain Mast Cell-Specific Proteases. J Immunol 2016;197:3382-92

16. Ekstrom EJ, Bergenfelz C, von Bulow V, Serifler F, Carlemalm E, Jonsson G, et al. WNT5A induces release of exosomes containing pro-angiogenic and immunosuppressive factors from malignant melanoma cells. Mol Cancer 2014;13:88

17. Mincheva-Nilsson L, Baranov V. Cancer exosomes and NKG2D receptor-ligand interactions: impairing NKG2D-mediated cytotoxicity and anti-tumour immune surveillance. Semin Cancer Biol 2014;28:24-30

18. Belting $\mathrm{M}$, Christianson HC. Role of exosomes and microvesicles in hypoxia-associated tumour development and cardiovascular disease. J Intern Med 2015;278:251-63

19. Kucharzewska P, Christianson HC, Welch JE, Svensson KJ, Fredlund E, Ringner M, et al. Exosomes reflect the hypoxic status of glioma cells and mediate hypoxia-dependent activation of vascular cells during tumor development. Proceedings of the National Academy of Sciences of the United States of America 2013;110:7312-7

20. Thery C, Amigorena S, Raposo G, Clayton A. Isolation and characterization of exosomes from cell culture supernatants and biological fluids. Curr Protoc Cell Biol 2006; Chapter 3:Unit 322 
21. van der Vlist EJ, Nolte-'t Hoen EN, Stoorvogel W, Arkesteijn GJ, Wauben MH. Fluorescent labeling of nano-sized vesicles released by cells and subsequent quantitative and qualitative analysis by highresolution flow cytometry. Nat Protoc 2012;7:1311-26

22. Groot Kormelink T, Arkesteijn GJ, Nauwelaers FA, van den Engh G, Nolte-'t Hoen EN, Wauben MH. Prerequisites for the analysis and sorting of extracellular vesicle subpopulations by high-resolution flow cytometry. Cytometry A 2016;89:135-47

23. Rouschop KM, Dubois LJ, Keulers TG, van den Beucken T, Lambin P, Bussink J, et al. PERK/elF2alpha signaling protects therapy resistant hypoxic cells through induction of glutathione synthesis and protection against ROS. Proc Natl Acad Sci U S A 2013;110:4622-7

24. Iancu CV, Tivol WF, Schooler JB, Dias DP, Henderson GP, Murphy GE, et al. Electron cryotomography sample preparation using the Vitrobot. Nat Protoc 2006;1:2813-9

25. Nath S, Dancourt J, Shteyn V, Puente G, Fong WM, Nag S, et al. Lipidation of the LC3/GABARAP family of autophagy proteins relies on a membrane-curvature-sensing domain in Atg3. Nat Cell Biol 2014;16:41524

26. Sou YS, Tanida I, Komatsu M, Ueno T, Kominami E. Phosphatidylserine in addition to phosphatidylethanolamine is an in vitro target of the mammalian Atg8 modifiers, LC3, GABARAP, and GATE-16. J Biol Chem 2006;281:3017-24

27. Tadokoro H, Umezu T, Ohyashiki K, Hirano T, Ohyashiki JH. Exosomes derived from hypoxic leukemia cells enhance tube formation in endothelial cells. J Biol Chem 2013;288:34343-51

28. Le Grand JN, Bon K, Fraichard A, Zhang J, Jouvenot M, Risold PY, et al. Specific distribution of the autophagic protein GABARAPL1/GEC1 in the developing and adult mouse brain and identification of neuronal populations expressing GABARAPL1/GEC1. PLoS One 2013;8:e63133

29. Weidberg H, Shvets E, Shpilka T, Shimron F, Shinder V, Elazar Z. LC3 and GATE-16/GABARAP subfamilies are both essential yet act differently in autophagosome biogenesis. Embo J 2010;29:1792-802

30. Klionsky DJ, Abdelmohsen K, Abe A, Abedin MJ, Abeliovich H, Acevedo Arozena A, et al. Guidelines for the use and interpretation of assays for monitoring autophagy (3rd edition). Autophagy 2016;12:1-222

31. Schaaf MB, Cojocari D, Keulers TG, Jutten B, Starmans MH, de Jong MC, et al. The autophagy associated gene, ULK1, promotes tolerance to chronic and acute hypoxia. Radiother Oncol 2013;108:529-34

32. Boyer-Guittaut M, Poillet L, Liang Q, Bole-Richard E, Ouyang X, Benavides GA, et al. The role of GABARAPL1/GEC1 in autophagic flux and mitochondrial quality control in MDA-MB-436 breast cancer cells. Autophagy 2014;10:986-1003

33. McAfee $Q$, Zhang Z, Samanta A, Levi SM, Ma XH, Piao S, et al. Autophagy inhibitor Lys05 has single-agent antitumor activity and reproduces the phenotype of a genetic autophagy deficiency. Proc Natl Acad Sci U S A 2012;109:8253-8

34. Yang A, Rajeshkumar NV, Wang X, Yabuuchi S, Alexander BM, Chu GC, et al. Autophagy is critical for pancreatic tumor growth and progression in tumors with p53 alterations. Cancer Discov 2014;4:905-13

35. Keulers TG, Schaaf MB, Rouschop KM. Autophagy-Dependent Secretion: Contribution to Tumor Progression. Front Oncol 2016;6:251

36. Umezu T, Tadokoro H, Azuma K, Yoshizawa S, Ohyashiki K, Ohyashiki JH. Exosomal miR-135b shed from hypoxic multiple myeloma cells enhances angiogenesis by targeting factor-inhibiting HIF-1. Blood 2014;124:3748-57

37. Ramteke A, Ting H, Agarwal C, Mateen S, Somasagara R, Hussain A, et al. Exosomes secreted under hypoxia enhance invasiveness and stemness of prostate cancer cells by targeting adherens junction molecules. Mol Carcinog 2015;54:554-65

38. Zomer A, Maynard C, Verweij FJ, Kamermans A, Schafer R, Beerling E, et al. In Vivo imaging reveals extracellular vesicle-mediated phenocopying of metastatic behavior. Cell 2015;161:1046-57 


\section{Supplementary figures}

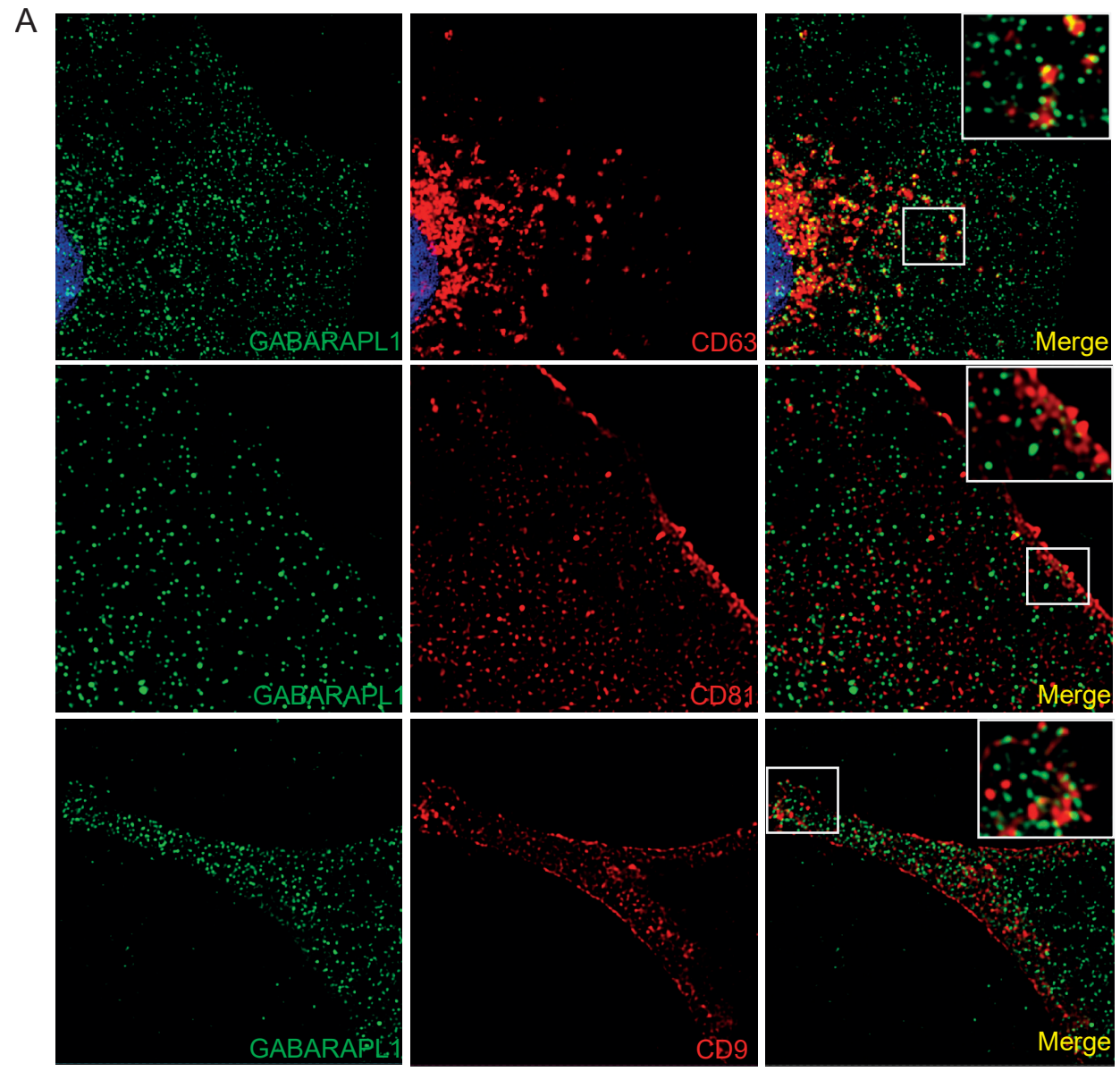

Suppl fig 1

(A) GABARAPL1 (Green) is associated with EV markers CD63 (red), CD81 (red) and CD9 (red) in U87 cells. Nucleus is depicted in blue. 
A
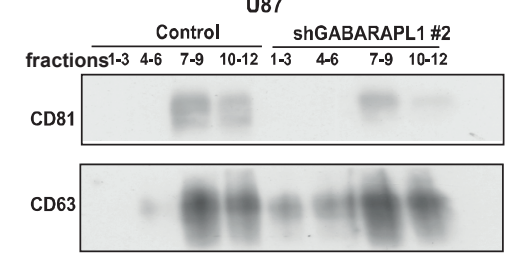

$\operatorname{CDg}$

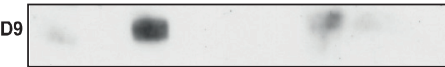

B

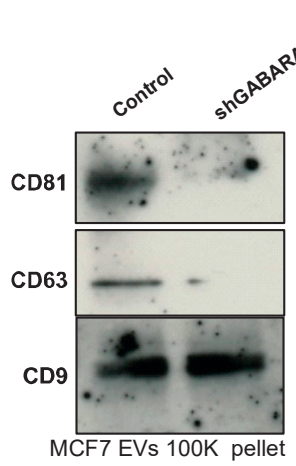

HT29

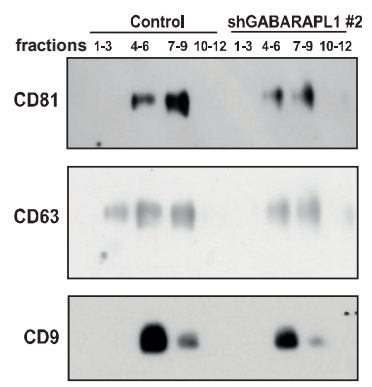

D

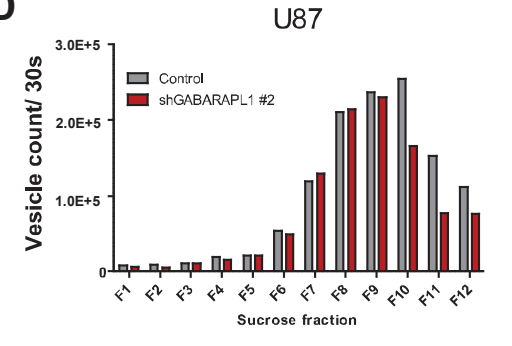

U87

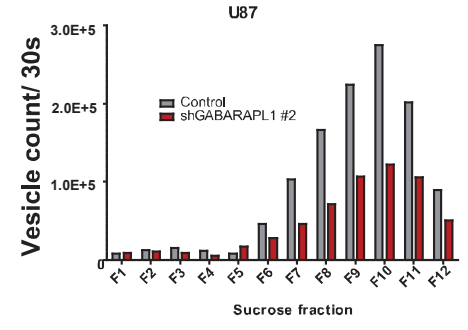

C

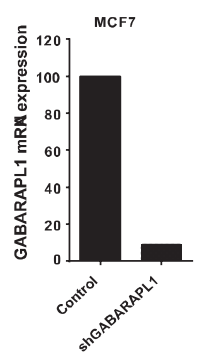

U87
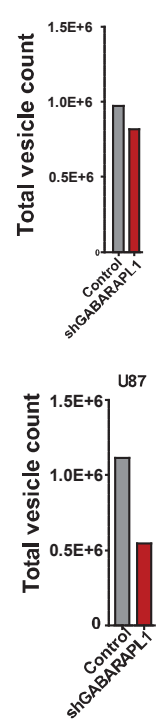

\section{Suppl fig 2}

(A) Western blots of isolated EVs from U87 and HT29 control and GABARAPL1 knockdown expressing an independent hairpin. Fractions are pooled per 3. (B) Western blot analysis of the 100k pellet of GABARAPL1 deficient MCF7 cells show reduced marker expression. (C) GABARAPL1 mRNA expression of MCF7 GABARAPL1 knockdown cells. (D) Quantification of EVs secreted by U87 cells with an independent hairpin. Shown are 2 biological repeats. 
A

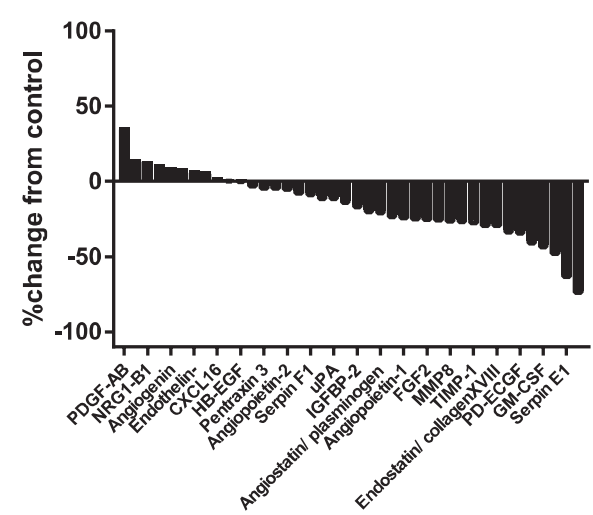

MCF7 hypoxia

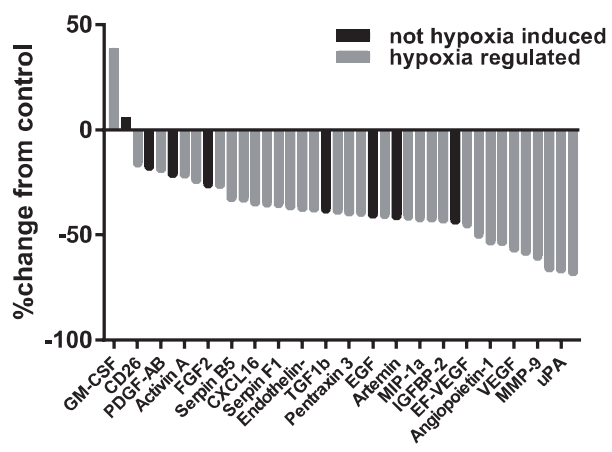

Suppl fig 3

(A) Quantification of antibody arrays of MCF7 control and GABARAPL1 knockdown cells.

A

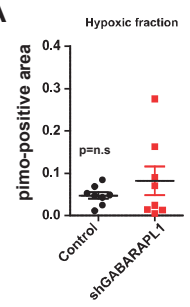

B

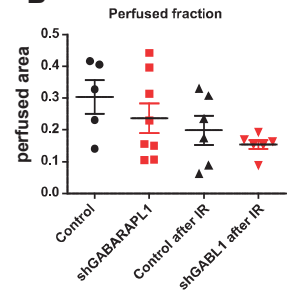

C

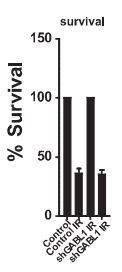

D
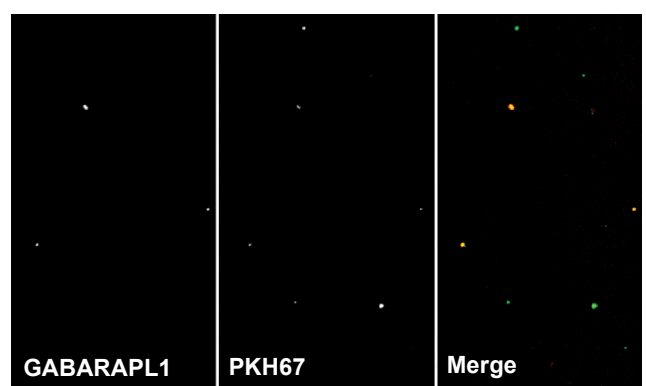

Suppl fig 4

(A) GABARAPL1 knockdown does not change the hypoxic fraction or (B) perfusion in U87 xenografts. (C) Clonogenic survival assays show that GABARAPL1 is not required for cell survival after irradiation $(n=3)$. 


\section{Supplementary information Materials and methods}

\section{Sample processing for Quantitative Mass-spectrometry}

After 24 hours normoxia or hypoxia exposure, conditioned culture medium (serum free) was collected and spun down for 10 min 300xg. Subsequently, secreted proteins were concentrated using centrifugal filters (Amicon Ultra, 3K). Protein concentration was determined using Bradford assay (Bio Rad) Secreted proteins were lysed by sonication in $12 \mathrm{mM}$ Sodiumdeoxycholate (SDC),12 mM sodium laurylsarcosinate (SLS) in $100 \mathrm{mM}$ Tris/HCl (pH 9.0). (Method modified from Masuda et al Mol Cell Proteomics, 2009,8(12), 2770-2777). Disulfide bonds were reduced in $5 \mathrm{mM}$ dithiothreitol at $50^{\circ} \mathrm{C}$ for 30 minutes and free sulfhydryl groups were then alkylated in $10 \mathrm{mM}$ iodoacetamide for $30 \mathrm{~min}$ at room temperature in the dark. The protein mixture was diluted 5 times with $50 \mathrm{mM}$ ammonium bicarbonate and digested with trypsin at $37^{\circ} \mathrm{C}$ overnight. Digestions were acidified with trifluoroacetic acid(TFA) to a concentration of $0.5 \%$ to precipitate the SDC and SLS. SDC and SLS were removed by centrifugation.

Desalting and reductive dimethylation of free amino groups was performed on C18 stagetips (Method modified from Wilson-Grady et al Volume 61, Issue 3, 15 june 2013, Pages 277-286). Briefly Tryptic peptides from secreted proteins were separately loaded onto stagetips and washed with $200 \mu \mathrm{l} 0.1 \%$ TFA and subsequently with $200 \mu \mathrm{l} 0.25 \mathrm{M}$ MES (2-(N-morpholino) ethanesulfonic acid) pH 5.5. Samples 1L: MCF7 SCR N, 3L: MCF7 GABL1 A, 4L: HT29 SCR A and 6L:HT29 GABL1 A were labelled by loading $200 \mu$ light labeling solution (0.4\% formaldehyde (Sigma) and $60 \mathrm{mM}$ sodium cyanoborohydride (Sigma) in 0.25 M MES (pH 5.5) onto the stagetip. Samples 2H: MCF7 Control hypoxia and $5 \mathrm{H}$ : HT29 control hypoxia were labelled by loading $200 \mu$ l heavy labelling solution.(0.4\% ${ }^{13} \mathrm{C}$-D2 formaldehyde (Sigma Isotec) and $60 \mathrm{mM}$ (D3)-sodium cyanoborodeuteride (Sigma Isotec) in 0.25 M MES (pH 5.5). The stagetips were washed with $200 \mu \mathrm{l} 0.1$ $\%$ Formic acid and labelled peptides were eluted with $100 \mu \mathrm{l} 50 \%$ acetonitrile with 0.1 $\%$ Formic acid. Next eluates from samples $1 \mathrm{~L}+2 \mathrm{H}, 2 \mathrm{H}+3 \mathrm{~L}, 4 \mathrm{~L}+5 \mathrm{H}$ and $5 \mathrm{H}+6 \mathrm{~L}$ were mixed and dried by vacuum centrifugation.

\section{Mass spectrometry analysis}

All LC-MS/MS analyses were performed on an Q Exactive mass spectrometer (Thermo Scientific, San Jose, CA). Dried mixed light and heavy labeled samples were resuspended in $0.5 \%$ Formic Acid in 3\% Acetonitrile and loaded onto an Easy spray column (Pepmap RSLC, C18, 2 um, 75 um x 25 cm Thermo Fisher Scientific). Peptides were separated using the Thermo Fisher Scientific Easy Nano LC1000 (buffer A=0.1\% formic acid and buffer $\mathrm{B}=0.1 \%$ formic acid in acetonitrile) using a gradient of $0-28 \%$ buffer $\mathrm{B}$ for 90 min, with a flow rate of $300 \mathrm{nl} / \mathrm{min}$. Each Data collection cycle in the Q Exactive consisted of 1 full MS scan (300-1750 m/z) followed by 15 data dependent MS/MS scans. 


\section{Bioinformatic analysis}

Raw Data files were processed using MaxQuant software suite (version 1.5.2.8, Tyanova, S.; Temu, T.; Cox, J. The MaxQuant computational platform for mass spectrometry based shotgun proteomics. Nature Protoc. 2016, 11 (12), 2301-2319) for identification and relative quantification of proteins. A false discovery rate (FDR) of 0.01 for proteins and peptides and a minimum peptide length of six amino acids were required. The Andromeda search engine was used to search the MS/MS spectra against the Human Uniprot database. A maximum of two missed cleavages were allowed. Q Exactive spectra were analyzed using MaxQuant's default settings for Orbitrap spectra, including a main search peptide and MS/MS match tolerance of 4.5 and 20 ppm, respectively. The maximum precursor ion charge state used for searching was 7, and the enzyme specificity was set to trypsin. Further modifications were cysteine carbamidomethylation (fixed) as well as protein methionine oxidation. For demethylation labeled samples 28.031 Da on lysine and the peptide N-terminus (light) and 36.076 Da on lysine and the peptide Nterminus (heavy) were added to the search parameters. The minimum number of razor and unique peptides was set to 1 . Heavy-to-light $(\mathrm{H} / \mathrm{L})$ ratios were calculated using MaxQuant's default settings. 


\section{Chapter}

\section{GABARAPL1 is essential for metastasis formation in mice}

Tom G. Keulers ${ }^{1}$, Imke Demers ${ }^{1}$, Sten Libregts ${ }^{2}$, Kim Savelkouls ${ }^{1}$, Joris J. Roelofs ${ }^{3}$, Johan Bussink$^{4}$, Marijke I. Zonneveld ${ }^{1}$ Marca Wauben², Kasper M. Rouschop ${ }^{1}$

${ }^{1}$ Department of Radiotherapy, GROW - School for Oncology and Developmental Biology,

Maastricht University Medical Centre+, Maastricht, The Netherlands

${ }^{2}$ Department of Biochemistry \& Cell Biology, Faculty of Veterinary Medicine, Utrecht University, Utrecht, The Netherlands.

${ }^{3}$ Department of Pathology, Academic Medical Center, University of Amsterdam, Amsterdam, The Netherlands.

${ }^{4}$ Department of Radiation Oncology, Radboud University Medical Center, Nijmegen, The Netherlands. 


\section{Abstract}

Metastasis formation contributes to most cancer related deaths and is a major clinical challenge. Tumour hypoxia contributes to metastasis formation by eliciting a metastatic phenotype mediated by hypoxia responsive pathways such as the HIF pathway and the unfolded protein response (UPR). Previously we showed that during hypoxia, GABARAPL1 is required for the secretion of a subset of extracellular vesicles with proangiogenic and tumour growth promoting capacities. This subset is marked by GABARAPL1 expression on the EV surface (GABARAPL1+EV). Here we explored to role of GABARAPL1 in metastasis formation, and observed that silencing GABARAPL1 drastically reduced lung metastasis development. Our data suggests that GABARAPL1 ${ }^{+}$EVs are required for the formation of pre-metastatic niches. Silencing GABARAPL1 led to changes in EV composition characterized by increased EV marker expression and increased total protein, suggesting that GABARAPL1 is involved in EV-cargo sorting required for metastasis development. Although this study describes preliminary data, we show that GABARAPL1 is essential for metastasis formation, which might be mediated through GABARAPL1 dependent EV secretion. However, further research is needed to confirm these observations. 


\section{Introduction}

Most cancer related deaths are attributed to metastatic diseases and is therefore a major clinical challenge in cancer therapy. Metastasis is a complex cascade that requires tumour cells that have obtained specific cellular characteristics. During the metastatic process, tumour cells leave the primary tumour through the lymphatic and blood circulation (intravasation) to disseminate throughout the body. Via the circulatory system these circulating tumour cells (CTC) invade the distant organs. Regions within specific organs with favourable microenvironment, allow docking and growth of distant tumours, the pre-metastatic niche $[1,2]$. In contrast to metastatic niches, which are formed by CTC after extravasation of the circulation, pre-metastatic niches are characterized by a tumour growth favouring microenvironment before the arrival of CTC [1, 2]. EVs are phospholipid bilayer enclosed vesicles (50-500 $\mathrm{nm}$ ) that contain bioactive molecules such as nucleic acids (DNA, RNAs, miRNAs), lipids and proteins (receptors, enzymes, transcription factors and extracellular matrix proteins) but are devoid of cellular organelles [3]. Most cells secrete extracellular vesicles (EVs) as a vehicle of intercellular communication. EVs highly enriched in tetraspanin expression (CD81, CD63 and CD9), which are widely used as markers for EV identification. Extracellular vesicles (EVs) are shown to contribute to cancer progression and metastasis formation [4] and contribute to the formation of the pre-metastatic niche by transferring cargo that changes the microenvironment of acceptor cells in a metastasis favouring way. For instance cancer cells promote extravasation by destroying the endothelial barrier (and thereby induce vascular leakiness) through the EV mediated delivery of miR-105 which regulates the tight junction protein ZO-1 [5]. Other parts of the multi-step metastatic process are, at least in part mediated by EVs, such as intravasation (through induction of EMT)[6], angiogenesis [7] and matrix remodelling [8].

Tumour hypoxia, or low oxygen tension, is a unique feature of solid tumours which promotes metastasis and is associated with increased carcinogenesis and therapy resistance [9]. Cells respond to hypoxia by activating hypoxia responsive pathways such as the HIF pathway and the unfolded protein responds (UPR) that regulate expression of genes that promote the metastatic phenotype. HIF dependent signalling is shown to contribute to metastasis through epithelial mesenchymal transition (EMT) $[10,11]$ inducing migration and invasion and development of the pre-metastatic niche (reviewed in [12] [13] [14, 15].

Recently, we discovered a specific sub-type of pro- angiogenic EVs generated during hypoxia. These GABARAPL1+EVs display pro-tumoural properties and contribute to tumour progression by inducing angiogenesis (chapter 6 ). It was shown before that EVs derived from hypoxic cells elicit a pro-metastatic phenotype by stimulating HIF dependent pathways [16], have immune modulating capacities by impairing NK cell function [17] and contribute to ECM remodelling [18].

In this study we explore the role of GABARAPL1 ${ }^{+} E V$ in the metastatic cascade. 


\section{Materials and methods}

\section{Cell culture}

MDA-MB-231 breast cancer cells were cultured in DMEM (LONZA) supplemented with 10\% FCS. Hypoxia exposure (16-20 hours over night) was done using a modular atmosphere controlled system (Don Whitley Scientific, United Kingdom). GABARAPL1 knockdown was achieved by lentiviral transduction of shGABARAPL1 expression (TRCN0000419975, ACAGTGATGAGAGTGTCTATG) in pLKO-tet-on backbone. pLKO-teton-stuffer was taken as a control. For EV isolation experiments, cells were cultured in EV depleted medium (100.000 RCF overnight)

\section{$R T-q P C R$}

RNA extraction was done using Nucleospin RNA kit (Macherey-Nagel) and cDNA was prepared using I-script (Bio-Rad). Real-time PCR was done using ABI-7500 real-time PCR (Applied Biosystems). Gene abundance was measured using SYBR green (Thermos Fisher). Abundance of each gene was normalized to $18 \mathrm{~s}$.

\section{Tumour models}

Inducible shGABARAPL1 and Empty vector control MDA-MB-231 cells were injected in the mammary fat pad of female NMRI ${ }^{\text {nu/nu }}$ mice $\left(1.0 \times 10^{6}\right.$ cells/50ul matrigel (Corning)). Doxycycline was administered via the drinking water ( $2 \mathrm{~g} / \mathrm{L}$, $5 \%$ sucrose). Tumour size was assessed by calliper measurements in 3 dimensions. Animals were injected with BrdU (30 mg/ kg, Sigma Aldrich) and pimonidazole (60 mg/ kg, Hypoxyprobe) intraperitoneally 1 hour prior to sacrifice. Hoechst was injected intravenous $3 \mathrm{mg} / \mathrm{kg} 1$ minute before killing. When tumours reached $1000 \mathrm{~mm}^{3}$, animals were sacrificed and tumours and lungs were dissected and paraffin embedded. Metastases were visualized using human cytokeratin18/HRP (Dako) staining and scored on 3 different levels (2 $\left.\begin{array}{llll}2 & 0 & \mu \mathrm{m}\end{array}\right)$ per lung and counted manually.

GFP expressing control cells and RFP expressing shGABARAPL1 cells were orthotopi-

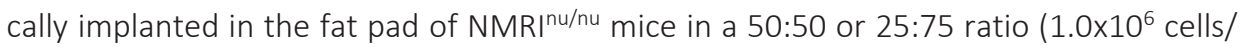
$50 u l$ matrigel (Corning). When tumours reached $1000 \mathrm{~mm} 3$ mice were killed and lungs were removed and snap-frozen in liquid nitrogen. Metastases were visualized using fluorescent microscopy.

All animal experiments were conducted in accordance with national guidelines and approved by the animal ethics committee Maastricht University.

\section{Immunohistochemistry and image processing}

Frozen acetone fixed cross sections were stained using Pimonidazole (Chemicon), 9F1 Rat MAb to mouse endothelium, (Radboud University Nijmegen, Dept of Pathology) as 
described previously [19]. Slides were quantified by computerized digital image processing system using fluorescent microscope with a computer-controlled motorized stepping stage, equipped with a high-res intensified solid-state camera (Axioskop; Zeis). Necrosis was examined morphologically by H\&E staining. Tumour hypoxia and (perfused) vascular densities are expressed as ratio to the total tumour area.

\section{EV isolation}

Equal amounts of control and shGABARAPL1 expressing cells were seeded ( $5 \times 10^{6}$ cells/ plate) in complete medium. After 24 hours, medium was replaced with EV depleted medium (100.000 RCF, overnight) containing 6\% FCS. Cells were exposed to hypoxia overnight. Conditioned medium was collected and EVs were isolated by differential ultracentrifugation: 200 RCF (10 min) 2000 RCF (20 min) 16.000 RCF (30 min) and 100.000 RCF (65 min). The 100.000 RCF pellet was resuspended in EV depleted $0.2 \%$ BSA in PBS. The EV pellet was PKH67 labelled (Sigma Aldrich) and loaded on the bottom of a linear sucrose gradient (2.0M-0.4M) for subsequent floatation. Gradients were made using a Gradient master system (Biocomp). Gradients were centrifuged at 200.000 RCF (16 hours). Gradients were separated in 12 fractions (bottom-up) and analysed by High-resolution flow-cytometry or immunoblot. For functional assays size exclusion chromatography was used: columns were prepared as described elsewhere [20]. Sepharose CL-2B (GE healthcare, Uppsala Sweden) and TELOS columns (10 ml) were used. For EV isolation, medium was collected and centrifuged at 200 RCF (10 min) 2000 RCF (20 min) 16.000 RCF (30 min). Supernatant was concentrated using Amicon Ultra Spin Concentrators (100 kDa cut off). Sample ( $\pm 500 u l)$ was loaded and collected in 12 fractions of $0.5 \mathrm{ml}$. EV RNA was isolated using miRNeasy micro kit (Qiagen) and analysed using the Bioanalzyer (Agilent)

\section{Immunoblotting}

Cells were lysed in RIPA buffer (50mM Hepes-KOH, pH 7.5, 150mM KCL, $1 \mathrm{mM}$ EDTA, $2 \mathrm{mM}$ mercaptoethanol, $0.2 \%$ Tween-29 with protease inhibitor (Roche) and sonicated 3 x 10 seconds at $10 \mathrm{MHz}$. Samples were separated by SDS-PAGE and transferred to PVDF membranes. Tetraspanins were lysed in non-reducing conditions.CD63 (1:1000, BD Biosciences) CD81 (1:1000, BD biosciences) LC3B (1:1000, PM036, MBL) Actin (1:200.000, MD bioscience) (GABARAPL1 110101- AP Protein tech group) antibodies were used as primary antibodies. Antibodies were visualized using HRP linked secondary antibodies (anti-Rabbit, anti-mouse, 1:2000, Cell signalling). Super Signal west pico chemiluminescent substrate (pierce biotechnology, Thermo Scientific) was used for detection. 


\section{microBCA}

Sucrose gradient fractions were pooled and pelleted at 100.000xRCF and pellets were resuspended in $\mathrm{PBS} / 2 \% \mathrm{SDS} / 1 \%$ triton. Protein concentration was determined according to the manufacturers manual. (Micro BCA, Greiner Bio-One)

\section{Fluorescence microscopy}

Images were taken from live-cell experiments. CD63-EGFP was expressed in C1 backbone vectors and visualized during culture conditions $\left(37^{\circ} \mathrm{C} 5 \% \mathrm{CO} 2\right)$ and using LEICA SP8 modular confocal microscope with resonant scanner.

\section{Migration assay}

20.000 Cells were seeded in a 96 well Image lock plate (Essen Bioscience). 700-800 micron wide scratches were made with the IncuCyte Wound maker (Essen Bioscience). Cell migration was monitored using the IncuCyte (Essen Bioscience). Images were taken with 2 hour intervals for 24 hours. Images were analysed for wound with, relative wound density and confluence using the IncuCyte software. Cells stimulated with EVs were incubated $\mathrm{O} / \mathrm{N}$ with $100 \mathrm{ul}$ size exclusion chromatography purified EVs derived from $3 \times 10^{6}$ MDA-MB 231 cells. Medium flow through was used as a control.

\section{Results}

GABARAPL1 contributes to metastasis formation in mice

Previously we showed that during hypoxia, a subpopulation of EVs is dependent on GABARAPL1 for secretion. Mass spectrometry analysis of the GABARAPL1 dependent secretome revealed that 10 of top 20 GABARAPL1-dependent secreted factors are associated with metastasis formation and EVs (table 1). These findings stimulated us to investigate if GABARAPL1+EVs are required for metastasis formation. To determine if GABARAPL1 is involved in metastasis formation in vivo, we engineered a metastatic breast cancer cell line (MDA-MB-231) with doxycycline inducible GABARAPL1 knockdown (fig $1 \mathrm{~A}$ ) and implanted them orthotopically in the fat-pad of female $\mathrm{NMRi} \mathrm{i}^{\mathrm{Nu} / \mathrm{Nu}}$ mice. Knockdown was induced by adding doxycycline to the drinking water $(2 \mathrm{~g} / \mathrm{L})$.

When tumours reached their maximum size $\left(1000 \mathrm{~mm}^{3}\right)$ lungs were removed. Cross sections were stained for human cytokeratin18 (fig 1B) to detect metastasis on 3 levels of the lungs ( $200 \quad 0 \mu m$ distance). Interestingly, the number of lung metastasis in mice bearing GABARAPL1 knockdown tumours decreased by $84 \%$ (fig 1C). 
A

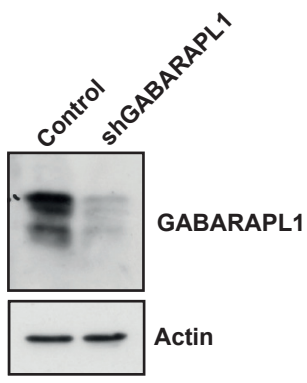

D

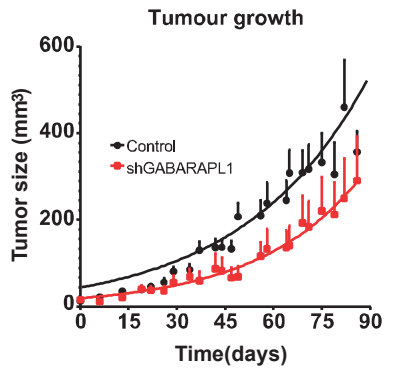

B

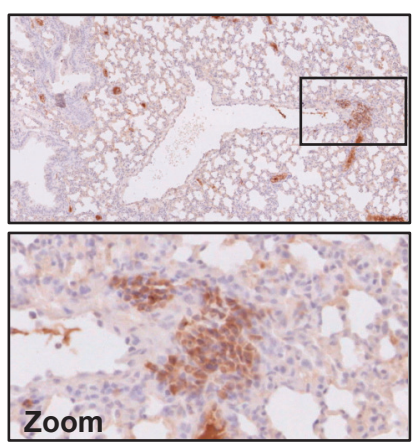

C

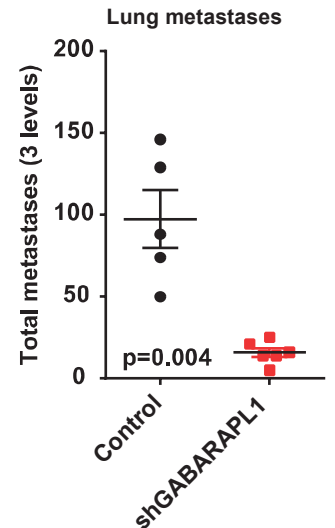

E

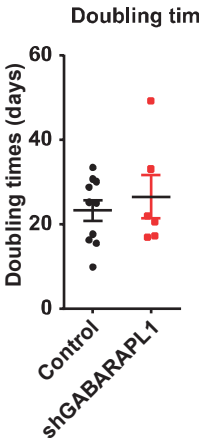

$\mathbf{F}$ Palpable tumour $\left(>100 \mathrm{~mm}^{3}\right)$

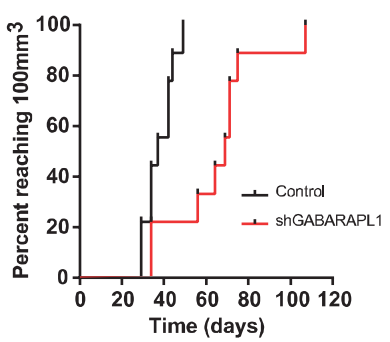

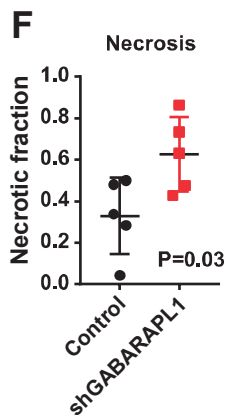
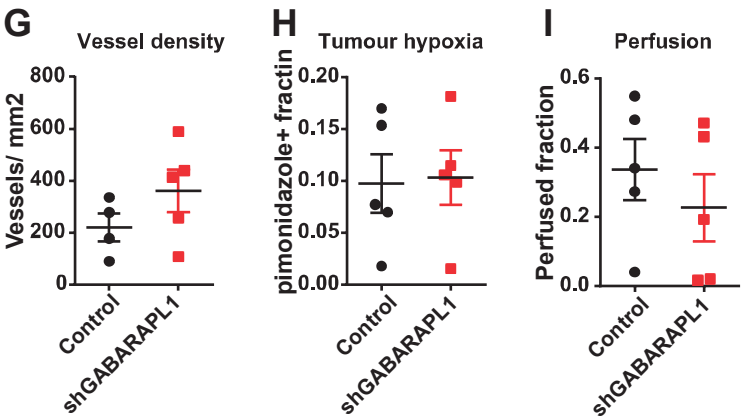

Figure 1: GABARAPL1 contributes to metastasis formation in mice.

(A) Immunoblots of MDA-MD-231 Control and GABARAPL1 knockdown cells after doxycycline exposure. (B) Lung metastasis visualized by cytokeratin18 staining. (C) Quantification of lung metastases of mice with control and shGABARAPL1 xenografts. Plotted is total metastasis detected in 3 levels per mouse. Mean \pm SEM. $p=0.004$, student's t-test, unpaired). (D) Growth curve of control and shGABARAPL1 xenografts. (E) Doubling times of individual control and GABARAPL1 knockdown xenografts. (F) Kaplan-meier plot of control and shGABARAPL1 tumours reaching $100 \mathrm{~mm}^{3}$. (F) Tumour necrosis of MDA-MB-231 xenografts was examined morphologically by H\&E staining (error bars \pm SEM, $p=0.03$, students' $t$-test). (G) Vessel density as determined by $9 F 1$ immunohistochemistry (error bars \pm SEM). Hypoxic fraction xenografts was determined using pimoni- 
dazole immunohistochemistry (error bars \pm SEM). I) Vessel perfusion of control and GABARAPL1 knockdown xenografts, determined by Hoechst staining (error bars \pm SEM).

Table 1. 10 of the top 20 less secreted proteins during hypoxia after GABARAPL1 knockdown associated with EV (www.exocarta.org) and metastasis.

\begin{tabular}{llll}
\hline Protein name & EV associated & Detected in EV derived/isolated from & $\begin{array}{l}\text { Metastasis } \\
\text { association }\end{array}$ \\
\hline Heat shock protein HSP 90-beta & Yes & Mast cells & {$[21,22]$} \\
CD81 antigen & Yes & a general EV marker & {$[23]$} \\
Stanniocalcin-2 & Yes & Mesenchymal stem cell & {$[24-26]$} \\
Sulfhydryl oxidase 1 & Yes & Colorectal cancer, detected in urine & {$[27]$} \\
Protein S100-A11 & Yes & Colorectal cancer & {$[28,29]$} \\
Keratin, type I cytoskeletal 18 & Yes & Oligodendrocytes & {$[30]$} \\
Laminin subunit alpha-5 & Yes & Colorectal cancer & {$[31]$} \\
Heat shock protein 27 beta-1 & Yes & Bladder- breast cancer, saliva, Urine & {$[32-42]$} \\
Nucleophosmin & Yes & Detected in urine & {$[43,44]$} \\
Stress-induced-phosphoprotein 1 & Yes & Bladder cancer & {$[45,46]$} \\
\hline
\end{tabular}

In line with previous results (chapter 6), GABARAPL1 knockdown tumours display decreased tumour growth (fig $1 \mathrm{D}-\mathrm{F}$ ) and increased tumour necrosis (fig 1G). Other microenvironmental factors (hypoxic fraction vasculature, and perfusion (fig 1 G-I)) do not differ between controls and GABARAPL1 knockdown xenografts.

\section{GABARAPL1 modulates EV composition in MDA-MB-231 cells}

We previously showed that GABARAPL1 is required for EV secretion and EV composition in colorectal cancer (HT29) and glioblastoma cells (U87) (chapter 6). To characterize the secreted GABARAPL1 dependent EV population of MDA-MB-231 cells, we quantified single secreted EVs by high-resolution flow cytometry of control and GABARAPL1 knockdown cells exposed to normoxia, moderate $\left(0.2 \% \mathrm{O}_{2}\right)$ hypoxia or severe hypoxia $\left(<0.02 \% \mathrm{O}_{2}\right)$ (fig $\left.2 \mathrm{~A}\right)$. After exposure of $16 \mathrm{~h}$ in previously EV-depleted medium, EVs were isolated by sequential ultracentrifugation and further purified by sucrose density flotation. Gradients were divided in 12 fractions and assessed individually. Quantification of total secreted EV revealed no difference in EVs secreted by control and GABARAPL1 deficient cells (fig 2B). In parallel, total protein of secreted EVs was determined by Micro-cinchoninic acid assay (micro-BCA). Interestingly, although total secreted EV counts do not differ between control and GABARAPL1 knockdown cells, EVs secreted by GABARAPL1 deficient cells display decreased total protein content (fig 2C). In addition, immunoblot analysis revealed increased in general EV markers (CD63 and CD81) of EVs released by GABARAPL1 knockdown cells (fig 2D). These data indicate that lack of GABARAPL1 changes the overall EV phenotype without direct effects on total EV secretion in MDA-MB-231 cells. These data suggest that GABARAPL1 is involved in loading and cargo sorting of EVs rather than the secretory processes or transport. To determine 
if the sorting effect is restricted to protein or also on RNA inclusion, total EV RNA was extracted and RNA yield and size distribution was analysed using through Bioanalyzer determination. Interestingly, EVs released by GABARAPL1 deficient cells contain different RNA fragments than control EVs (area under the peak), suggesting more larger RNA fragments in GABARAPL1 knockdown cells (fig 2E).
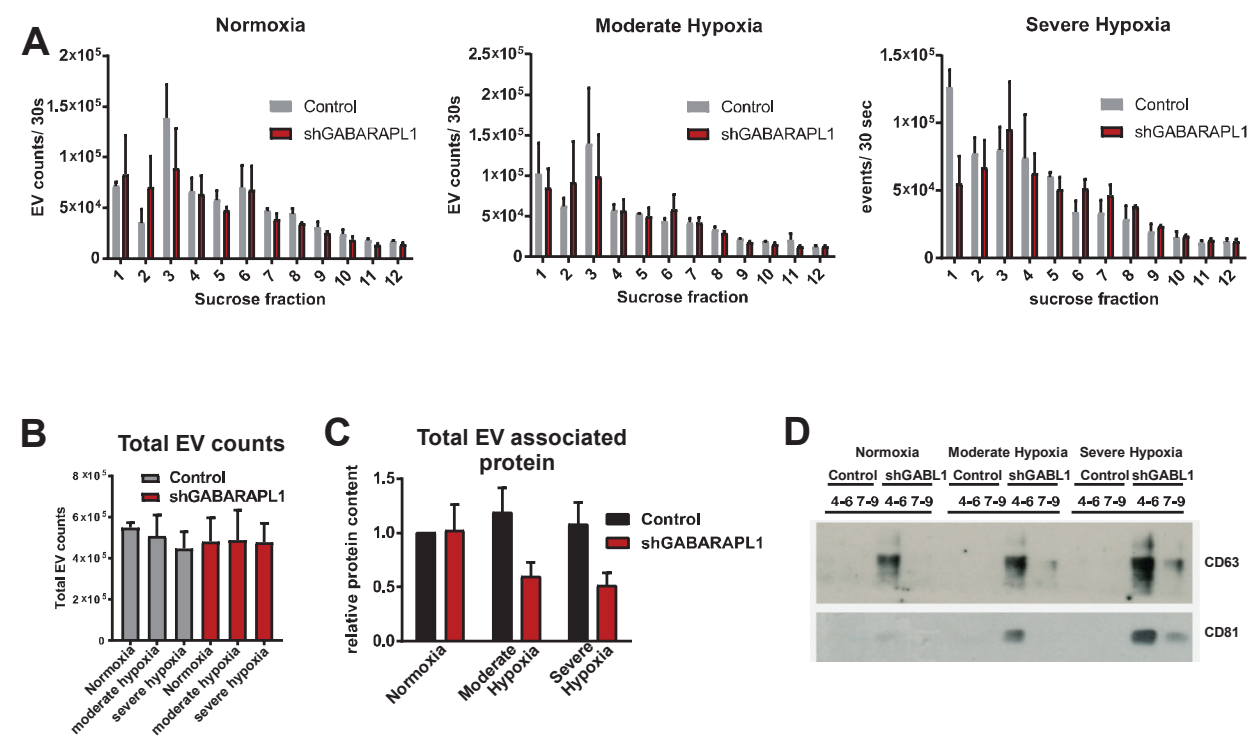

E

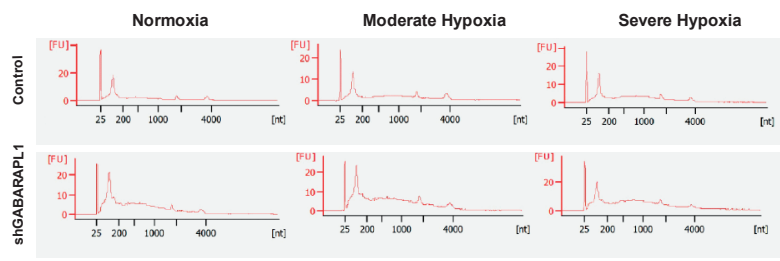

$\mathbf{F}$
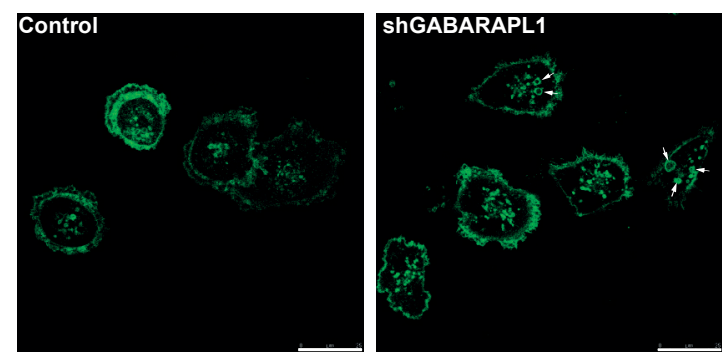

Figure 2: GABARAPL1 modulates EV composition in MDA-MB-231 cells.

(A) High-resolution flow cytometry analysis of sucrose fractions (12 fractions per sample) of MDA-MB-231 control and shGABARAPL1 knockdown cells exposed to normoxia $\left(20 \% \mathrm{O}_{2}\right.$, moderate hypoxia $\left(0.2 \% \mathrm{O}_{2}\right)$ and severe hypoxia $\left(0.02 \% \mathrm{O}_{2}\right)$. (B) Total counts of high-resolution flow cytometry. (C) Total protein content of isolated EVs from control and shGABARAPL1 cells exposed to normoxia $\left(20 \% \mathrm{O}_{2}\right)$, moderate hypoxia $\left(0.2 \% \mathrm{O}_{2}\right)$ and severe hypoxia $\left(0.02 \% \mathrm{O}_{2}\right)$. (D) Corresponding immunoblots of EV markers (CD63 and CD81) of sucrose 
fractions of MDA-MB-231 control and shGABARAPL1 knockdown cells exposed to normoxia $\left(20 \% \mathrm{O}_{2}\right)$, moderate hypoxia $\left(0.2 \% \mathrm{O}_{2}\right)$ and severe hypoxia $\left(0.02 \% \mathrm{O}_{2}\right)$. shGABL1 = shGABARAPL1 (E) Bioanalyzer RNA profiles of EVs isolated from of MDA-MB-231 control and shGABARAPL1 knockdown cells exposed to normoxia $\left(20 \% \mathrm{O}_{2}\right)$ moderate hypoxia $\left(0.2 \% \mathrm{O}_{2}\right)$ and severe hypoxia $\left(0.02 \% \mathrm{O}_{2}\right)$. (F) MDA-MB-231 control and GABARAPL1 knockdown cells expressing CD63-eGFP.

Sorting of cargo is mediated by ECSRT proteins during endosome maturation. Late endosomes/ Multivesicular bodies, typically marked by CD63 expression, are fated for degradation or can fuse with the plasma membrane to release their intraluminal vesicles (referred to as exosomes). Enlargement of CD63-eGFP positive intracellular compartments (MVB/ late endosomes) in GABARAPL1 deficient cells suggests defects in endosomal pathways (fig 2F).

\section{GABARAPL1 mediates cell migration}

Metastasis formation is the result of a sequential series of events including invasion, intravasation into the vasculature, extravasation into the target tissue and colonization. To test if GABARAPL1 is involved in cell migration, migration studies using scratch assay were performed. These studies show that MDA-MB-231 GABARAPL1 deficient cells display decreased migratory capacity (fig $3 \mathrm{~A}$ ). To test if this effect can be transferred by EVs, migration studies were performed with wild-type MDA-MB-231 cells stimulated with EVs isolated from MDA-MB-231 control and GABARAPL1 knockdown which were exposed to normoxia, moderate hypoxia and severe hypoxia (fig 3B-C). Although EVs derived from severe hypoxic GABARAPL1 knockdown cells do induce a statistical significant decreased migratory capacity after 22 hours, the biological significant effect of EVs on wild-type cell migration remains to be elucidated.

GABARAPL1 deficient cells have a decreased capacity to metastasize to the lungs (fig 1A). To determine whether these effects are due to cell intrinsic changes (i.e. the inability to invade, migrate or intravasate) or related to secretory-associated mechanism such as the pre-metastatic niche formation, we implanted xenografts with mixed populations of MDA-MB-231 control and GABARAPL1 knockdown cells expressing GFP and RFP respectively. When tumours reached the maximum size $\left(1000 \mathrm{~m}^{3}\right)$, lungs were removed and cross sections were examined for RFP and/or GFP expressing metastasis (figure 4. Interestingly, both control and ShGABARAPL1 metastases were detected in the lungs, however shGABARAPL1 RFP expressing metastases were always accompanied by GFP expressing control cells. Although these data need to be confirmed by additional experiments, they support the hypothesis that GABARAPL1 is required for (pre-)metastatic niche formation. 
A
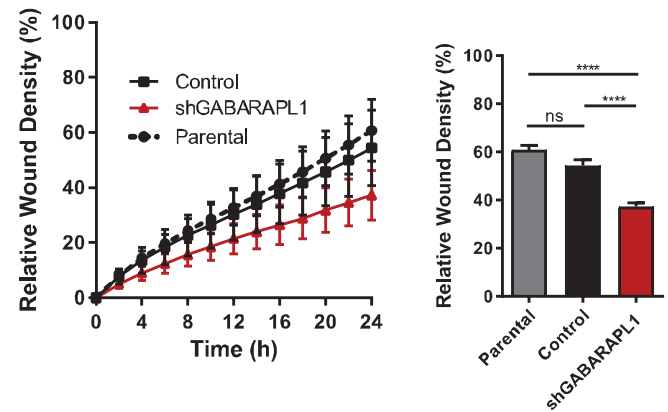

B

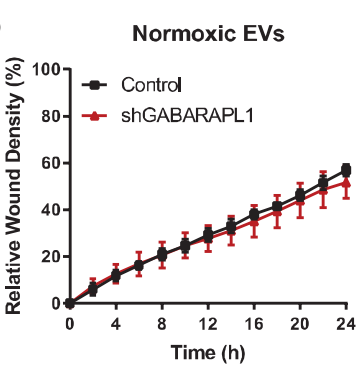

C
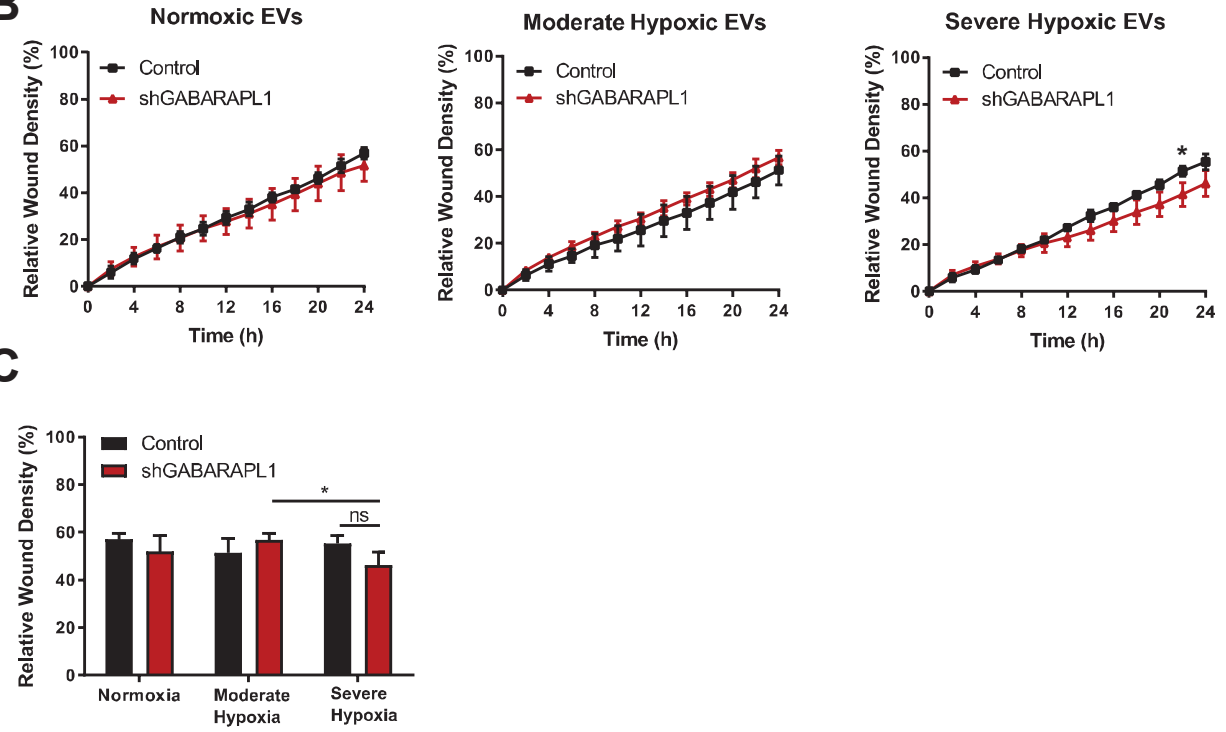

Figure 3: GABARAPL1 mediates cell migration.

(A) Migration rate of MDA-MB-231 parental, control and GABARAPL1 knockdown cells. (B) Migration rates of parental MDA-MB-231 cells stimulated with EVs derived from control and GABARAPL1 knockdown cells exposed to normoxia $\left(20 \% \mathrm{O}_{2}\right)$ moderate hypoxia $\left(0.2 \% \mathrm{O}_{2}\right)$ and severe hypoxia $\left(0.02 \% \mathrm{O}_{2}\right)$. (C) Relative wound density after 24 hours cell migration. 
A

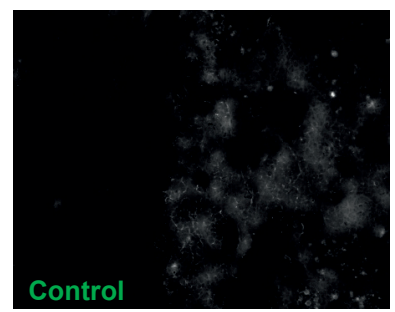

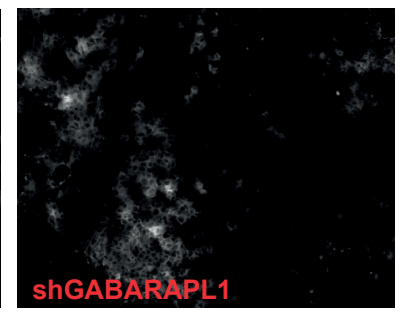

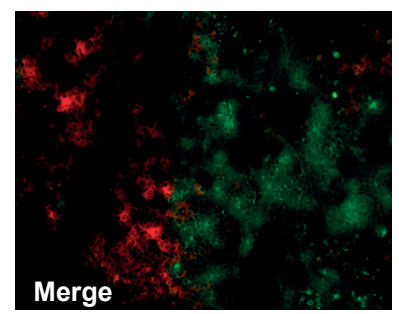

B
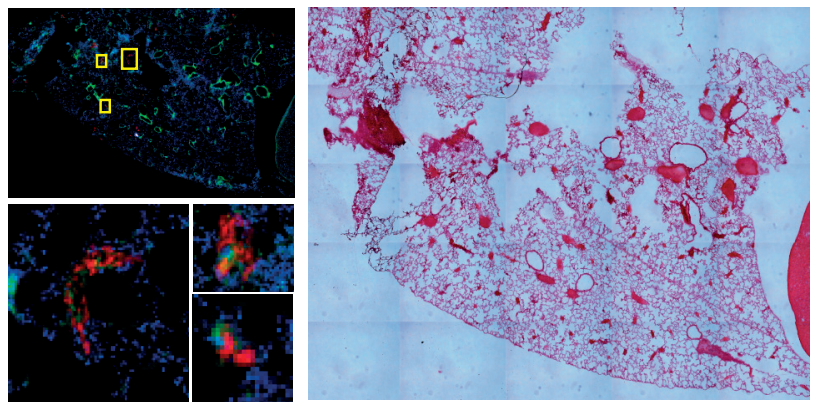

Figure 4: GABARAPL1 and pre-metastatic niche formation.

(A) Cross section of mixed population xenograft containing GFP expressing control cells (Green) and RFP expressing GABARAPL1 knockdown cells (Red). Nuclei are depicted in blue. (B) Cross section of the lung depicting control (green) and shGABARAPL1 (red) metastases with matching H\&E staining.

\section{Discussion}

Previously we showed that GABARAPL1+EV contribute to tumour progression by inducing angiogenesis. In this study we show that GABARAPL1 contributes to metastasis formation, presumably through EV secretion and development of the pre-metastatic niche. In vitro experiments revealed that, in line with previous studies (chapter 7), GABARAPL1 is involved in cargo sorting and EV loading. MDA-MB-231 GABARAPL1 deficient cells are in fact able to secrete EVs, but the composition is changed in a GABARAPL1 dependent manner. Changes in EV composition are characterized by a significant increase in EV markers CD63 and CD81 although the total protein content is strongly decreased. If these proteins are structural proteins or sorted cargo remains to be determined. Furthermore, GABARAPL1 ${ }^{+} E V$ s contain more larger RNA fragments, presumably mRNAs compared to control EVs, which contain more small RNA, presumably miRNA's, fragments.

The formation of metastasis is a sequence of events. After cells detached from the primary tumour, they survive and extravasate the circulation and invade and colonize the target tissue. In this study we investigated the role of GABARAPL1 in the formation of metastasis, with a focus on GABARAPL1 dependent EV secretion. That GABARAPL1 is required for the development of metastasis we showed in an orthotopic model were we 
found that GABARAPL1 deficient cells have a decreased metastatic capacity. This decreased capacity resulted in a decrease (-80\%) of lung metastasis. In agreement with previous studies in HT29 and U87 xenografts, MDA-MB-231 GABARAPL1 deficient tumours show an increase in tumour necrosis, probably due to a decreased stress tolerance in the tumour microenvironment. This could in part explain the decrease in metastasis due to a decrease in viable and potentially migratory cells. However the increase in necrosis is not in proportion to the decrease in metastasis found in the lungs. Cell migration is crucial in the metastasis development. In MDA-MB-231 cells, GABARAPL1 seems to play a role in cell migration. This effect seems to be partially transferred by EVs in a GABARAPL1 dependent manner, although the effect is minimal.

Accumulating evidence shows that formation of the pre-metastatic niche contributes to metastasis development. The pre-metastatic niche provides a pro-tumorigenic microenvironment for CTC to invade, survive and grow in distant tissue. During premetastatic niche formation the interaction between BMDC, struma and tumour cells is critical. It was previously shown that during pre-metastatic niche formation BMDC are recruited to the pre-metastatic niche through secretion of tumour derived secreted factors. Recent studies indicated that EVs play an important role in the communication between tumour cells and BMDC required for pre-metastatic niche formation [47]. Other tumour derived secreted factors such as LOX family protein's LOX, LOXL2 and LOXL4, SDF4, VEGF, MMPs[48] [49] and the MET oncoprotein [47] are also shown to contribute directly or indirectly to pre-metastatic niche formation. Previous studies done by us (chapter 7) showed that secretion of some of these factors (LOXL4, SDF4, VEGF and MET) are GABARAPL1 dependent. To test if GABARAPL1 contributes to premetastatic niche formation we grew tumours orthotopically with mixed populations of GFP-expressing control and RFP/shGABARAPL1 expressing MDA-MB-231 cells. The rationale of this experiment is that control cells are still able to initiate pre-metastatic niches. If the previously found difference in lung metastasis formation is a result of cell intrinsic changes, only GFP (control) expressing metastasis will be found, since GABARAPL1 deficient cells are not able to metastasize. Concordantly, if the effect is due to defects in secretion of tumour derived secreted factors of GABARAPL1 knockdown cells, RFP and GFP expressing metastasis will be observed. In this study we traced RFP and GFP expressing metastasis back in the lungs, however RFP expressing GABARAPL1 knockdown cells were always accompanied by GFP expressing control cells. These studies support the hypothesis that GABARAPL1 deficient cells indeed cannot form a premetastatic niche, probably due to defects in secretion of tumour derived secreted factors. However, these data are based on preliminary results and need to be validated by additional experiments. 


\section{Acknowledgements}

The authors would like to thank Hans Peters his excellent technical support. This work was financially supported by the Dutch Cancer Society (KWF Grants UM 2012-5506 and 2015-7735 to K.R.), worldwide cancer research award 16-0265 (to K.R.) 


\section{References}

1. Psaila, B. and D. Lyden, The metastatic niche: adapting the foreign soil. Nature reviews. Cancer, 2009. 9(4): p. 285-93.

2. Kaplan, R.N., et al., VEGFR1-positive haematopoietic bone marrow progenitors initiate the pre-metastatic niche. Nature, 2005. 438(7069): p. 820-7.

3. Raposo, G. and W. Stoorvogel, Extracellular vesicles: exosomes, microvesicles, and friends. The Journal of cell biology, 2013. 200(4): p. 373-83.

4. Becker, A., et al., Extracellular Vesicles in Cancer: Cell-to-Cell Mediators of Metastasis. Cancer cell, 2016. 30(6): p. 836-848.

5. Zhou, W., et al., Cancer-secreted miR-105 destroys vascular endothelial barriers to promote metastasis. Cancer cell, 2014. 25(4): p. 501-15.

6. Holzel, M., A. Bovier, and T. Tuting, Plasticity of tumour and immune cells: a source of heterogeneity and a cause for therapy resistance? Nature reviews. Cancer, 2013. 13(5): p. 365-76.

7. Grange, C., et al., Microvesicles released from human renal cancer stem cells stimulate angiogenesis and formation of lung premetastatic niche. Cancer research, 2011. 71(15): p. 5346-56.

8. Shimoda, M. and R. Khokha, Metalloproteinases in extracellular vesicles. Biochimica et biophysica acta, 2017. 1864(11 Pt A): p. 1989-2000.

9. Vaupel, P. and A. Mayer, Hypoxia in cancer: significance and impact on clinical outcome. Cancer metastasis reviews, 2007. 26(2): p. 225-39.

10. Yang, M.H., et al., Direct regulation of TWIST by HIF-1alpha promotes metastasis. Nature cell biology, 2008. 10(3): p. 295-305.

11. Kim, W.Y., et al., HIF2alpha cooperates with RAS to promote lung tumorigenesis in mice. The Journal of clinical investigation, 2009. 119(8): p. 2160-70.

12. Rankin, E.B. and A.J. Giaccia, Hypoxic control of metastasis. Science, 2016. 352(6282): p. 175-80.

13. Erler, J.T., et al., Hypoxia-induced lysyl oxidase is a critical mediator of bone marrow cell recruitment to form the premetastatic niche. Cancer cell, 2009. 15(1): p. 35-44.

14. Erler, J.T. and A.J. Giaccia, Lysyl oxidase mediates hypoxic control of metastasis. Cancer research, 2006. 66(21): p. 10238-41.

15. Erler, J.T., et al., Lysyl oxidase is essential for hypoxia-induced metastasis. Nature, 2006. 440(7088): p. 1222-6.

16. Li, L., et al., Exosomes Derived from Hypoxic Oral Squamous Cell Carcinoma Cells Deliver miR-21 to Normoxic Cells to Elicit a Prometastatic Phenotype. Cancer research, 2016. 76(7): p. 1770-80.

17. Berchem, G., et al., Hypoxic tumor-derived microvesicles negatively regulate NK cell function by a mechanism involving TGF-beta and miR23a transfer. Oncoimmunology, 2016. 5(4): p. e1062968.

18. de Jong, O.G., et al., Exosomes from hypoxic endothelial cells have increased collagen crosslinking activity through up-regulation of lysyl oxidase-like 2. Journal of cellular and molecular medicine, 2016. 20(2): p. 342-50.

19. Rouschop, K.M., et al., PERK/elF2alpha signaling protects therapy resistant hypoxic cells through induction of glutathione synthesis and protection against ROS. Proceedings of the National Academy of Sciences of the United States of America, 2013. 110(12): p. 4622-7.

20. Boing, A.N., et al., Single-step isolation of extracellular vesicles by size-exclusion chromatography. Journal of extracellular vesicles, 2014. 3.

21. Biaoxue, R., et al., Upregulation of Hsp90-beta and annexin A1 correlates with poor survival and lymphatic metastasis in lung cancer patients. Journal of experimental \& clinical cancer research : CR, 2012. 31: p. 70.

22. Wang, J., et al., High expression of heat shock protein 90 is associated with tumor aggressiveness and poor prognosis in patients with advanced gastric cancer. PloS one, 2013. 8(4): p. e62876.

23. Mazzocca, A., F. Liotta, and V. Carloni, Tetraspanin CD81-regulated cell motility plays a critical role in intrahepatic metastasis of hepatocellular carcinoma. Gastroenterology, 2008. 135(1): p. 244-256 e1. 
24. Kita, Y., et al., STC2: a predictive marker for lymph node metastasis in esophageal squamous-cell carcinoma. Annals of surgical oncology, 2011. 18(1): p. 261-72.

25. Pena, C., et al., STC1 expression by cancer-associated fibroblasts drives metastasis of colorectal cancer. Cancer research, 2013. 73(4): p. 1287-97.

26. Volland, S., et al., Stanniocalcin 2 promotes invasion and is associated with metastatic stages in neuroblastoma. International journal of cancer. Journal international du cancer, 2009. 125(9): p. 204957.

27. Katchman, B.A., et al., Expression of quiescin sulfhydryl oxidase 1 is associated with a highly invasive phenotype and correlates with a poor prognosis in Luminal B breast cancer. Breast cancer research : BCR, 2013. 15(2): p. R28.

28. Meding, S., et al., Tissue-based proteomics reveals FXYD3, S100A11 and GSTM3 as novel markers for regional lymph node metastasis in colon cancer. The Journal of pathology, 2012.

29. Nipp, M., et al., S100-A10, thioredoxin, and S100-A6 as biomarkers of papillary thyroid carcinoma with lymph node metastasis identified by MALDI imaging. Journal of molecular medicine, 2012. 90(2): p. 16374.

30. Kramer-Albers, E.M., et al., Oligodendrocytes secrete exosomes containing major myelin and stressprotective proteins: Trophic support for axons? Proteomics. Clinical applications, 2007. 1(11): p. 1446-61.

31. Hibino, S., et al., Identification of an active site on the laminin alpha5 chain globular domain that binds to CD44 and inhibits malignancy. Cancer research, 2004. 64(14): p. 4810-6.

32. Chen, J., et al., Proteome analysis of gastric cancer metastasis by two-dimensional gel electrophoresis and matrix assisted laser desorption/ionization-mass spectrometry for identification of metastasis-related proteins. Journal of proteome research, 2004. 3(5): p. 1009-16.

33. Gibert, B., et al., Targeting heat shock protein 27 (HspB1) interferes with bone metastasis and tumour formation in vivo. Br J Cancer, 2012. 107(1): p. 63-70.

34. Guo, K., et al., Regulation of HSP27 on NF-kappaB pathway activation may be involved in metastatic hepatocellular carcinoma cells apoptosis. BMC Cancer, 2009. 9: p. 100.

35. Nagaraja, G.M., P. Kaur, and A. Asea, Role of human and mouse HspB1 in metastasis. Curr Mol Med, 2012. 12(9): p. 1142-50.

36. Shiota, M., et al., Hsp27 regulates epithelial mesenchymal transition, metastasis, and circulating tumor cells in prostate cancer. Cancer Res, 2013. 73(10): p. 3109-19.

37. Song, H.Y., et al., [Proteomic analysis on metastasis-associated proteins of hepatocellular carcinoma tissues]. Zhonghua Gan Zang Bing Za Zhi, 2005. 13(5): p. 331-4.

38. Song, H.Y., et al., Proteomic analysis on metastasis-associated proteins of human hepatocellular carcinoma tissues. J Cancer Res Clin Oncol, 2006. 132(2): p. 92-8.

39. Wei, L., et al., Hsp27 participates in the maintenance of breast cancer stem cells through regulation of epithelial-mesenchymal transition and nuclear factor-kappaB. Breast Cancer Res, 2011. 13(5): p. R101.

40. Zhang, D., L.L. Wong, and E.S. Koay, Phosphorylation of Ser78 of Hsp27 correlated with HER-2/neu status and lymph node positivity in breast cancer. Molecular cancer, 2007. 6: p. 52.

41. Zhao, L., et al., Differential proteomic analysis of human colorectal carcinoma cell lines metastasisassociated proteins. J Cancer Res Clin Oncol, 2007. 133(10): p. 771-82.

42. Zhao, M., et al., Increased expression of heat shock protein 27 correlates with peritoneal metastasis in epithelial ovarian cancer. Reprod Sci, 2012. 19(7): p. 748-53.

43. Coutinho-Camillo, C.M., et al., Nucleophosmin, p53, and Ki-67 expression patterns on an oral squamous cell carcinoma tissue microarray. Hum Pathol, 2010. 41(8): p. 1079-86.

44. Liu, Y., et al., Expression of nucleophosmin/NPM1 correlates with migration and invasiveness of colon cancer cells. J Biomed Sci, 2012. 19: p. 53.

45. Walsh, N., et al., RNAi knockdown of Hop (Hsp70/Hsp90 organising protein) decreases invasion via MMP2 down regulation. Cancer Lett, 2011. 306(2): p. 180-9.

46. Walsh, N., et al., Identification of pancreatic cancer invasion-related proteins by proteomic analysis. Proteome Sci, 2009. 7: p. 3. 
47. Peinado, H., et al., Melanoma exosomes educate bone marrow progenitor cells toward a pro-metastatic phenotype through MET. Nature medicine, 2012. 18(6): p. 883-91.

48. Semenza, G.L., Hypoxia-inducible factors: mediators of cancer progression and targets for cancer therapy. Trends in pharmacological sciences, 2012. 33(4): p. 207-14.

49. Sceneay, J., M.J. Smyth, and A. Moller, The pre-metastatic niche: finding common ground. Cancer metastasis reviews, 2013. 32(3-4): p. 449-64. 

Chapter

Summary and general discussion 
Cancer is still causing great morbidity and mortality worldwide and as our population ages, the incidence rates are increasing. Cancer arises due to genetic changes that accumulate over cellular generations and cancer cells are selected to withstand the sometimes harsh conditions observed within the tumour. In addition, cancer cells use the same general mechanisms that normal cells utilize to adapt to (micro-) environmental changes.

Tumours are characterized by the presence of immature and often chaotic vasculature that results in insufficient blood supply to areas within the tumour. The high metabolic rate of cancer cells in combination with insufficient oxygen delivery results in hypoxic tumour regions, a common feature of most solid tumours. Oxygenation in tumours may vary from normal values ( $5 \%$ ) close to the vessel wall to complete anoxia $\left(0 \% \mathrm{O}_{2}\right)$ close to perinecrotic regions. Hypoxic cells are more resistant to chemo- and radiotherapy and are associated with increased metastasis resulting in poor prognosis of patients. Although common in tumours, hypoxia (especially severe hypoxia) is not observed in the human body and therefore poses a therapeutic opportunity. Fundamental understanding of how cancer adapts to hypoxic episodes is therefore essential to develop novel therapeutic strategies. The work in this thesis addresses novel findings how cancer cells respond to hypoxia. One of the mechanisms that allows cells to survive hypoxia is autophagy, consequently autophagy inhibition increases therapy efficacy in pre-clinical studies and cancer patients $(1,2)$ (+ chapter 4$)$. Autophagy is a mechanism that allows the cell to recycle aged or obsolete subcellular compartments/ organelles and protein aggregates to maintain cellular homeostasis. During normal conditions the autophagic rate is relatively low, but can be rapidly upregulated during periods of (metabolic) stress. In cancer cells elevated autophagy-activity is frequently observed. Autophagy is dependent on the formation of intracellular vesicles, the autophagosome. The cargo to be degraded is randomly or selectively sorted into these autophagosomes. The latter via specific adaptor proteins that recognize ubiquitylated substrates. These proteins act as an adaptor between the recognized cargo and members of the LC3/GABARAP protein family. This LC3/GABARAP protein family consists of 7 members including LC3A, -B and C, and GABARAP, GABARAPL1 and GABARAPL2, which share a high degree of homology in amino acid sequence and tertiary structure. The last decade most of autophagy research has been focused on LC3B. Yet, due to the high degree of homology and lack of unstructured overview the family members' function, the current dogma is that all LC3B/GABARAP protein family members are redundant and functionally involved in autophagy. In chapter 2, we systematically reviewed the current knowledge on the individual functional role of the LC3/ GABARAP protein family members. In addition to overlapping functions, most LC3/ GABARAP protein family members mediate unique, autophagy (in)dependent functions. Proteins from the GABARAP subfamily display several functions in intracellular trafficking. For example, GABARAP and GABARAPL1 are required for the trafficking and membrane expression of receptors, including the kappa opioid receptor, the angiotensin receptor II and the GABA receptor 
(3-5), but also the transient receptor potential cation channel subfamily $V$ member (TRPV). It is still unclear how these processes work exactly, but it is suggested that they might function as a scaffold protein between kinesin motor proteins and the transport vesicle (6). GABARAPL1 is also shown to interact with motor proteins, however the functional role is still under investigation. In addition, we identified a role for GABARAPL1 in membrane expression of the epidermal growth factor receptor (EGFR) during hypoxia (chapter 3). Interestingly, GABARAPL1 expression is induced during hypoxia, which suggests a specific function during these conditions. The upregulation is dependent on increased transcription orchestrated by the PERK-ATF4 arm of the unfolded protein response (UPR), a cellular stress responsive pathway that senses unfolded and misfolded proteins in the ER. Furthermore we observed that only GABARAPL1, and not GABARAPL2 mRNA expression was induced during hypoxia, indicating unique regulatory mechanisms that control expression and suggesting that both proteins are required to fulfill specific functions during these conditions.

Tumour hypoxia is an independent prognostic marker for treatment outcome, irrespective of the treatment modality. Because GABARAPL1 expression is induced during hypoxia, we tested if GABARAPL1 expression could be linked to treatment outcome. In a panel of head and neck cancer patients GABARAPL1 expression correlated with reduced local and loco-regional control of these patients. In addition to the physical presence of hypoxia that contributes to resistance to therapies, GABARAPL1-dependent membrane expression of tumour growth stimulating receptors, such as the EGFR, and the secretion of pro-angiogenic factors (chapter 7) can contribute to decreased treatment outcome. In contrast to our studies, other groups reported tumour suppressive functions of GABARAPL1 and correlated GABARAPL1 expression to increased survival (7). However, these studies addressed different tumour sites, suggesting tumour specific mechanisms. Summarizing, this suggests that GABARAPL1 expression could be linked to treatment outcome and potentially could be used as a prognostic biomarker.

In addition to their role in autophagy and intracellular trafficking of receptors, LC3/ GABARAP protein family members but also other proteins of the core autophagy machinery, have been associated to secretory mechanisms. In chapter 6 we summarized current knowledge about autophagy-dependent secretion and what the consequences are on tumour progression. In the classical ER-to-Golgi route, proteins are marked for secretion by a leader-peptide, which serves as a secretion signal. However, also proteins without this sequence can be secreted via alternative forms of unconventional secretion in an autophagy-dependent manner (8-10). Autophagy-dependent secreted factors are actively released and have pro-metastatic properties can stimulate proliferation via auto- and paracrine signalling but also have immune modulating properties that can promote the invasive phenotype.

Also defects in autophagy, such as defects in lysosome/ autophagosome fusion, can lead to secretion of the cargo in the extracellular environment. The concept autophagydependent secretion is still in its infancy and is poorly understood. Many questions 
remain unanswered: How is the autophagy machinery involved in the biogenesis of secretory vesicles/ exosomes? What differentiates secretory vesicles from degradative vesicles? Where do these machineries converge? What is the signal for secretion?

After molecules have been internalized though several mechanisms of endocytosis, the internalized cargo are sorted in vesicular compartments referred to as endosomes after which they can have several different fates. Endosomes can be degraded though fusion with autophagosomes, or directly via fusion with lysosomes, but they can also be recycled back to the plasma membrane via formation of multivesicular bodies (MVB) which arise after intraluminal vesicles (ILV) formation by inward budding of the endosomal membrane. At this point, different sorting mechanisms, such as the ESCRT machinery are involved in fine-tuning the sorted cargo into these ILVs. Once MVBs fuse with the plasma membrane, ILVS are released in the extracellular environment as exosomes and are considered as extracellular vesicles.

Extracellular vesicles are emerging as vehicles of intercellular communication and more is known about their role in physiological and pathological conditions such as cancer. EVs are small membranous particles which are secreted by most, if not all cells and contain biological information such as miRNAs and proteins. In general, EV content seems to reflect the status of the cell of origin, however, accumulating evidence shows that specific molecules are enriched in EV subpopulations. In chapter 7, we demonstrate that during severe hypoxia, cells secrete a subpopulation of EVs that are characterized by a difference in size. This subpopulation is secreted in a GABARAPL1 dependent manner. These EVs, which are marked by GABARAPL1 expression on EV membrane, have pro-angiogenic properties that stimulate tumour growth. Depletion of GABARAPL1 through the expression of shRNAs alters EV composition in a cell line dependent manner. Xenografts of these GABARAPL1 knockdown cells displayed reduced growth, reduced vessel density and increased necrosis. However, the effect of GABARAPL1 depletion on EV secretion and composition seems to be cell line dependent. In HT29, GABARAPL1 depletion leads to decreased marker expression and decreased total EV counts. In contrast, in U87, GABARAPL1 depletion leads to decreased expression of some makers, but an increase in the tetraspanin CD63. Also total secreted EV counts were not affected. GABARAPL1 deficient MDA-MB-231 cells (chapter 8) show an increase in the tested markers CD81 and CD63 and differences in RNA loading. Currently, the mechanism how GABARAPL1 participates in EV secretion is under investigation. However, current data suggests that GABARAPL1 is involved in sorting of EV cargo rather than transport. This is supported by the observation that GABARAPL1 deficient cells have enlarged endosomal (CD63+) compartments (presumable late endosomes/ MVBs), which indicates defects in endosomal pathways. In HT29 cells, this sorting mechanism is probably disturbed to such an extensive degree, that also total EV secretion is decreased.

LC3/ GABARAP subfamily members can be conjugated to phosphatidylethanolamine (PE) at the C-terminal site to associate with membranes. For LC3 subfamily members, 
this conjugation is required for autophagy. GABARAPL1 also contains this C-terminal glycine, but this site is not required for GABARAPL1 mediated EV secretion (chapter 7). That GABARAPL1 is membrane bound, we demonstrated with cryo-transmission electron microscopy experiments, where we showed that after secretion, GABARAPL1 expression is retained at the EV surface. This might suggest that other post-translational mechanisms are at play to bind GABARAPL1 to the EV membrane. How this modification works and if this requires modification at the $\mathrm{N}$ or $\mathrm{C}$ terminal site, remains to be elucidated.

That GABARAPL1 expression is retained at the EV surface can be of therapeutic interest. In a small group of patients with glioblastoma, colorectal cancer and small cell lung cancer we detected elevated levels of GABARAPL1+EVs in plasma compared to healthy volunteers. As we demonstrated that GABARAPL1 ${ }^{+} E V$ s are secreted by hypoxic cells and are functionally active by stimulating angiogenesis and potentially mediating metastasis formation, this means too they could be explored as a therapeutic target or as biomarkers to determine the hypoxic levels of a tumour. That inhibition of GABARAPL1+EVs works to inhibit blood vessel formation we showed in vitro by preventing GABARAPL1+EV function with blocking antibodies to inhibit tube formation. Currently we are developing monoclonal antibodies to explore the therapeutical potential in vivo. In these studies we will explore tumour growth inhibition and prevention of metastasis. Furthermore, we will explore to potential of GABARAPL1+EVs as a biomarker to determine the hypoxic fraction of a tumour. Additionally, methods to determine GABARAPL1+EVs in the blood of cancer patients are being developed.

In addition to pro-angiogenic properties of GABARAPL1+EV, GABRAPL1+EVs are potentially involved in metastasis formation (chapter 8 ). We observed a decrease of $80 \%$ in lung metastasis in GABARAPL1 deficient orthotopically implanted breast tumours in mice, indicating an important role for GABARAPL1 in mediating metastasis formation. Metastasis is complex mechanisms were the cell require survival of multiple stages in the cascade. To develop into a metastasis, cells need to detach from the tumour, enter and survive the circulation and infiltrate the tissue and outgrow to a metastasis. Previously, we showed that GABARAPL1 $1^{+} E V$ have growth stimulating properties at distant tumour sites, we were therefore prompted to investigate the role of GABARAPL1 in metastasis development. Although this remains work in progress to elucidate the role of GABARAPL1 in metastasis formation, several steps in the metastatic cascade were addressed. GABARAPL1 seems to contribute to metastasis formation by development of the pre-metastatic niche. During development of the pre-metatatic niche, the micro milieu of the distant tissue is prepared in such a way that circulating tumour cells can colonize. To show this, we implanted breast tumours orthotopically in the fat pad of mice, consisting of a mixed population of Green fluorescent protein (GFP) expressing control cells, and Red fluorescent protein (RFP) expressing GABARAPL1 knockdown cells. After the tumour reached the maximum size, lungs were removed and lungs were examined for green and red fluorescent metastases. If the decrease in metastasis would 
be caused by cell intrinsic changes caused by GABARAPL1 knock down, such as decreased membrane expression of certain receptors, we expect to identify GFP expressing metastases only. If the decrease in metastases was caused by a defect in tumour derived secreted factors, such as EVs, we expected to identify control and GABARAPL1 deficient metastases in comparable numbers In the lungs we observed control cellderived metastases as well as GABARAPL1 knockdown derived metastases, which supports the hypothesis of a GABARAPL1 mediated pre-metastatic niche formation. Interestingly, RFP expressing GABARAPL1 knockdown cells were always accompanied by GFP expressing control cells, which also could suggest a metastatic niche formation by the control cells, rather than a pre-metastatic niche. Taken together we can conclude that GABARAPL1 is important for metastasis formation, and that this is potentially mediated through GABARAPL1+EV.

Epidermal growth factor receptor (EGFR) is a member of ErbB family of receptors and is expressed in normal tissues where it is involved in processes as proliferation and inhibition of apoptosis (11). Mutation or over expression of the receptor can lead to several pathologies such Alzheimer and cancer. EGFRvIII is a mutated form of EGFR characterized by a deletion of exon 2- 7 of the extracellular domain and is most prevalent in glioblastoma multiforme patients (GBM) who have a very poor prognosis $(12,13)$. As a consequence of deletion of the extracellular domain the receptor is constitutively active, promoting pro-oncogenic effects and tumourigenicity $(14,15)$. We demonstrated that EGFRvIII expressing tumours display increased activation and dependency on autophagy during metabolic stress including hypoxia. Typically EGFRvIII+ tumours show an increased tolerance towards hypoxia resulting in an increased hypoxic fraction. Additionally, EGFRvIII tumours show increased growth and increased regrowth rates after radiotherapy, resulting in decreased treatment outcome. Tumour hypoxia is a major limiting factor in cancer treatment (16). Therefore, targeting hypoxia in these tumours may contribute to increased survival rates. We demonstrate that EGFRvIII+ cells are more dependent on autophagy during hypoxia than non-EGFRvIII expressing tumours. Pharmacological inhibition of autophagy with the lysosomotropic drug chloroquine sensitizes EGFRvIII ${ }^{+}$cells to hypoxia and nutrient deprivation. As we have shown before, reducing the hypoxic fraction by targeting autophagy with chloroquine treatment sensitizes xenografts to radiotherapy (17). This effect is more pronounced in EGFRvIII+ expressing tumours. In a retrospective study we showed that patients with EGFRvIII+ GBM benefit most from concurrent chloroquine treatment. These results suggest that EG$\mathrm{FRvIII}^{+}$oncogene addiction in glioblastoma is a therapeutic vulnerability to chloroquine and could be a biomarker for patient stratification in clinical studies.

Strikingly, EGFRvIII+ cells consume more glucose suggesting that autophagy supports metabolic processes in EGFRvIII+ cells. The effects of autophagy inhibition on EGFRvIII positive cells, such as decreased hypoxia tolerance, can be rescued by pyruvate but not by glucose supplementation in the cell culture medium. This suggests that the glycolysis is already used to its fullest extent, and that autophagy is required as an additional en- 
ergy source for these tumours. These data are in agreement with previous studies, showing that other mutations in the RAS/RAF/MEK/ERK pathway, such the BRAFV600E mutation (1), but also HRAS ${ }^{G 12 V}$ or KRAS ${ }^{G 12 V}$ transformed cells show an increased dependency on autophagy (2).

Taken together, this study indicates that EGFRvIII+ have an increased metabolism and are more dependent on autophagy for their survival than EGFRvIII negative cells during metabolic stress. Remarkable results have been observed of EGFRvIII+ GBM patients treated concurrently with chloroquine and Radio- and chemotherapy (1). Currently 2 clinical trials (clinicaltrials.gov identifier NCT02378532 and NCT02432417) are initiated to evaluate toxicity of chloroquine in combination with radiotherapy and temozolamide, a chemotherapeutic commonly used for the treatment of GBM.

Autophagy degrades cyto- and genotoxic components, such as excessive ROS producing mitochondria, and is therefore considered a tumour suppressor mechanism. Several studies have demonstrated that defects in autophagy, due to deletions or mutations of proteins of the core autophagy machinery such as BECLIN1, can contribute to oncogenic transformation or at least neoplastic growth $(18)(19,20)$. The ATG12-ATG5 complex acts as an E3-like ubiquitin ligase and functions in lipidation of LC3/GABARAP family proteins and is required for autophagy. In our study we observed that expression of the autophagy associated protein ATG12 is lost in 40\% of tumours in a cohort of head and neck cancer patients. Interestingly, patients with tumour with lost ATG12 expression show improved survival and tumour control, especially in stage 3 and 4.

Strikingly, patients with ATG12 negative tumours had almost no hypoxic fraction and responded betters to therapies. It is common knowledge that autophagy inhibition sensitizes cells to internal and external stresses like hypoxia and nutrient deprivation, stressors which are common in the tumour micro environment. Therefore we hypothesized that defective autophagy (due to loss of ATG12 expression) sensitizes tumour cells to hypoxia leading to a decrease in tumour hypoxia, and since hypoxia is an important factor in therapy outcome, leading to improved therapy outcome. Indeed, in line with the observed effects in patients, xenografted ATG12 knockdown tumours do respond better to radiotherapy than their controls. However, this effect seems independent of the levels of hypoxia in these tumours as hypoxic fractions do not differ between control and knockdown groups. However, ATG12 deficient xenografts show a dramatically increase in tumour necrosis compared to control tumours, suggesting that these cells cannot survive in the tumour microenvironment due to stress exposure. Interestingly, ATG12 knockdown cells did not show an increased sensitization towards hypoxia alone, suggesting that a combination of stressors, such hypoxia and nutrient deprivation, is probably lethal. These combinations were not tested in the current study. The high fraction of ATG12 negative tumours prompted us to investigate if the loss of ATG12 expressing contributes to the oncogenic transformation of healthy cells. To test this we developed a model to specifically knockout ATG12 in the head and neck region and follow up tumour formation over time. This model was adapted from a previously de- 
scribed study were $75 \%$ of mice with SMAD4 ${ }^{-/}$in the head and neck had developed tumours in the oral cavity after 80 weeks (21). Therefore, SMAD4 ${ }^{-1-}$ was taken as a positive control. In contrast to the original study, we did not observe excessive tumour growth in the head and neck area in any group, although we found an increase in anomalies in the ATG12\% group compared to controls characterized by larger thymus, salivary glands and tongue. Although these observations still need to be confirmed by pathological examination, these findings support the hypothesis that loss of ATG12 expression contributes to malignant transformation. Furthermore, ATG12 negative tumours are sensitized to stresses in the tumour microenvironment, probably due to defects in autophagy, what contributes to improved therapy outcome. Taken together, these findings imply the use of ATG12 as a biomarker to predict tumour hypoxia and treatment outcome. Tumour hypoxia is an important factor in treatment outcome, and is therefore important to determine. Currently, tumour hypoxia is not integrated in therapy treatments due to the lack of reliable detection methods.

Other endogenous markers of hypoxia, such as HIF and its downstream targets GLUT-1 and CA9 are dependent on hypoxia for their expression and do not always reflect the spatio-temporal dynamics of hypoxia and are therefore not suitable for clinical applications $(22,23)$. In contrast to endogenous markers which aim for determining levels of hypoxia, ATG12 expressions seems to predict if a tumour is hypoxic, information which is relevant for treatment strategy.

Understanding mechanisms at play that allow tumour cells to survive hypoxia and adapt to the harsh environment is likely to result in future intervention possibilities. In this thesis we have addressed the complexity and versatile role of autophagy and autophagy-related proteins in tumorigenesis and tumour progression that based on our results may lead to novel prognostic tests and future treatment opportunities. 


\section{References}

1. Levy JM, Thompson JC, Griesinger AM, Amani V, Donson AM, Birks DK, et al. Autophagy inhibition improves chemosensitivity in BRAF(V600E) brain tumors. Cancer Discov 2014;4:773-80

2. Guo JY, Chen HY, Mathew R, Fan J, Strohecker AM, Karsli-Uzunbas G, et al. Activated Ras requires autophagy to maintain oxidative metabolism and tumorigenesis. Genes Dev 2011;25:460-70

3. Cook JL, Re RN, deHaro DL, Abadie JM, Peters M, Alam J. The trafficking protein GABARAP binds to and enhances plasma membrane expression and function of the angiotensin II type 1 receptor. Circ Res 2008;102:1539-47

4. Chen C, Wang Y, Huang P, Liu-Chen LY. Effects of C-terminal modifications of GEC1 protein and gammaaminobutyric acid type $A(G A B A(A))$ receptor-associated protein (GABARAP), two microtubule-associated proteins, on kappa opioid receptor expression. J Biol Chem 2011;286:15106-15

5. Lainez S, Valente P, Ontoria-Oviedo I, Estevez-Herrera J, Camprubi-Robles M, Ferrer-Montiel A, et al. GABAA receptor associated protein (GABARAP) modulates TRPV1 expression and channel function and desensitization. Faseb J 2010;24:1958-70

6. Nakajima K, Yin X, Takei Y, Seog DH, Homma N, Hirokawa N. Molecular motor KIF5A is essential for GABA(A) receptor transport, and KIF5A deletion causes epilepsy. Neuron 2012;76:945-61

7. Su W, Li S, Chen X, Yin L, Ma P, Ma Y, et al. GABARAPL1 suppresses metastasis by counteracting PI3K/Akt pathway in prostate cancer. Oncotarget 2017;8:4449-59

8. Dupont N, Jiang S, Pilli M, Ornatowski W, Bhattacharya D, Deretic V. Autophagy-based unconventional secretory pathway for extracellular delivery of IL-1beta. Embo J 2011;30:4701-11

9. Pallet N, Sirois I, Bell C, Hanafi LA, Hamelin K, Dieude M, et al. A comprehensive characterization of membrane vesicles released by autophagic human endothelial cells. Proteomics 2013;13:1108-20

10. Sirois I, Groleau J, Pallet N, Brassard N, Hamelin K, Londono I, et al. Caspase activation regulates the extracellular export of autophagic vacuoles. Autophagy 2012;8:927-37

11. Linggi B, Carpenter G. ErbB receptors: new insights on mechanisms and biology. Trends Cell Biol 2006;16:649-56

12. Wong AJ, Ruppert JM, Bigner SH, Grzeschik CH, Humphrey PA, Bigner DS, et al. Structural alterations of the epidermal growth factor receptor gene in human gliomas. Proc Natl Acad Sci U S A 1992;89:2965-9

13. Gan HK, Kaye AH, Luwor RB. The EGFRvIll variant in glioblastoma multiforme. J Clin Neurosci 2009;16:748-54

14. Theys J, Jutten B, Dubois L, Rouschop KM, Chiu RK, Li Y, et al. The deletion mutant EGFRvlll significantly contributes to stress resistance typical for the tumour microenvironment. Radiother Oncol 2009;92:399404

15. Learn CA, Hartzell TL, Wikstrand CJ, Archer GE, Rich JN, Friedman AH, et al. Resistance to tyrosine kinase inhibition by mutant epidermal growth factor receptor variant III contributes to the neoplastic phenotype of glioblastoma multiforme. Clin Cancer Res 2004;10:3216-24

16. Wouters BG, van den Beucken $T$, Magagnin MG, Lambin P, Koumenis C. Targeting hypoxia tolerance in cancer. Drug Resist Updat 2004;7:25-40

17. Rouschop KM, van den Beucken T, Dubois L, Niessen H, Bussink J, Savelkouls K, et al. The unfolded protein response protects human tumor cells during hypoxia through regulation of the autophagy genes MAP1LC3B and ATG5. J Clin Invest 2010;120:127-41

18. Liang C, Feng P, Ku B, Dotan I, Canaani D, Oh BH, et al. Autophagic and tumour suppressor activity of a novel Beclin1-binding protein UVRAG. Nat Cell Biol 2006;8:688-99

19. Qu X, Yu J, Bhagat G, Furuya N, Hibshoosh H, Troxel A, et al. Promotion of tumorigenesis by heterozygous disruption of the beclin 1 autophagy gene. J Clin Invest 2003;112:1809-20

20. Yue Z, Jin S, Yang C, Levine AJ, Heintz N. Beclin 1, an autophagy gene essential for early embryonic development, is a haploinsufficient tumor suppressor. Proc Natl Acad Sci U S A 2003;100:15077-82 
Chapter 9

21. Bornstein S, White R, Malkoski S, Oka M, Han G, Cleaver T, et al. Smad4 loss in mice causes spontaneous head and neck cancer with increased genomic instability and inflammation. J Clin Invest 2009;119:340819

22. Mayer A, Hockel M, Vaupel P. Endogenous hypoxia markers: case not proven! Adv Exp Med Biol 2008;614:127-36

23. Vordermark D, Brown JM. Endogenous markers of tumor hypoxia predictors of clinical radiation resistance? Strahlenther Onkol 2003;179:801-11 
Nederlandse samenvatting 
Kanker is een van de belangrijkste doodsoorzaken in de westerse samenleving. Kanker ontstaat door de accumulatie van mutaties in het DNA. De belangrijkste oorzaak van deze mutaties is blootstelling aan schadelijke stoffen van buitenaf, maar kunnen ook worden doorgegeven door overerving. Wanneer een cel genoeg mutaties heeft verworven in essentiële genen kunnen deze cellen niet meer reageren zoals het hoort en kan dit leiden tot ongecontroleerde celgroei waardoor een tumor ontstaat.

De groei van bloedvaten in een tumor draagt in belangrijke mate bij aan de ontwikkeling en groei van een tumor. Echter de vaatgroei in een tumor is in tegenstelling tot het vasculair systeem in gezond weefsel minder gestructureerd en meer chaotisch van aard. Mede hierdoor, en ook door de snelle groei van tumoren, komen er in een tumor gebieden voor die niet goed kunnen worden voorzien van zuurstof. Dit fenomeen heet hypoxie. Deze hypoxische gebieden zijn nadelig voor de prognose van een patiënt omdat deze gebieden in de tumor resistenter zijn tegen de meeste therapieën en dragen ze bij aan uitzaaiingen (metastasen). Omdat hypoxie elders in het lichaam niet voorkomt, liggen er ook kansen voor therapieën die specifiek zijn gericht op deze gebieden. Het werk in dit proefschrift beschrijft nieuwe bevindingen over hoe cellen zich gedragen tijdens hypoxie. Een van de mechanismen die cellen gebruiken om het gebrek aan zuurstof te overleven is autofagie. Autofagie is afgeleid uit het Grieks, en betekent zichzelf (auto) eten (fagie). Tijdens autofagie worden organellen en eiwitten afgebroken tot de essentiële bouwstenen zoals aminozuren, vetzuren en koolhydraten, waarna deze weer gebruikt kunnen worden voor de biogenese van onder andere nieuwe eiwitten die nodig zijn om de homeostase in de cel te bewaren. Autofagie is ook tijdens normale omstandigheden actief in de cel, maar wordt extra geactiveerd tijdens stress. Tijdens autofagie wordt het af te breken substraat ingepakt in een membraneus blaasje, het autofagosoom. Deze autofagosomen fuseren met lysosomen die de enzymen bevatten om de inhoud af te breken tot de biologische bouwstenen. Het af te breken substraat wordt herkend door zogenaamde adaptereiwitten die het substraat koppelen aan het autofagosoom via eiwitten van de LC3/GABARAP familie, die direct in het membraan van onder andere autofagosomen zijn verankerd. Tot nu toe zijn er zeven eiwitten van de LC3/GABARAP familie beschreven: LC3A (2 splicingvarianten), LC3B, LC3C, GABARAP, GABARAPL1 en GABARAPL2. Deze eiwitten van de LC3/GABARAP familie delen een hoge maten van homologie, echter zijn ze allen uniek in hun functie. Een uitgebreid literatuuronderzoek is beschreven in hoofdstuk $\mathbf{2}$ waar specifiek wordt ingaan op de individuele functies van elk van de eiwitten uit deze familie. Naast overeenkomsten, hebben LC3/ GABARAP eiwitten unieke autofagie afhankelijke en onafhankelijke functies. Zo is de GABARAP familie onder meer betrokken bij het transport van receptoren van en naar het plasmamembraan. Zo hebben wij aangetoond dat hypoxie specifiek de expressie van GABARAPL1, en geen andere eiwitten uit de LC3/GABARAP familie, induceert. Tijdens deze condities is GABARAPL1 nodig voor het tot expressie brengen van epidermale groeifactor receptor (EGFR) op het plasmamembraan (hoofdstuk 3). De toegenomen expressie 
van GABARAPL1 tijdens hypoxie wordt gereguleerd door de PERK-arm van de Unfolded protein response (UPR), een stressgereguleerde pathway die verkeerd- en ongevouwen eiwitten detecteert in het endoplasmatisch reticulum (ER) en daar op reageert.

Tumorhypoxie is een onafhankelijke prognostische indicator (marker) ongeacht de toegepaste therapie. Omdat GABARAPL1 verhoogd tot expressie komt tijdens hypoxie hebben we onderzocht of GABARAPL1 mRNA expressie gelinkt kan worden aan uitkomst van therapie. In een panel hoofd- halskankerpatiënten hebben we een correlatie aangetoond tussen GABARAPL1 expressie en verminderde tumorcontrole. Dit houdt in dat GABARAPL1 gebruikt zou kunnen worden als een biomarker om de uitkomst van therapie te voorspellen.

Epidermale groei factor receptor (EGFR) is een lid van de ErbB receptorfamilie en komt tot expressie in normale weefsels waar het is betrokken bij proliferatie en inhibitie van apoptose. Mutaties of overexpressie van deze receptor kunnen leiden tot verschillende aandoeningen zoals Alzheimer en kanker. EGFRvIII is een gemuteerde vorm van EGFR waarbij exon 2-7 zijn gedeleteerd en komt veelvuldig voor in glioblastoma multiforme (GBM). Doorgaans hebben GBM patiënten een zeer slechte prognose. Doordat het extracellulaire deel van de EGFR receptor is gedeleteerd, is de receptor constant actief waardoor groeisignalen continu worden doorgegeven, dat de groei van tumorcellen bevordert. EGFRvIII+ cellen zijn doorgaans resistenter tegen hypoxie, wat resulteert in een grotere hypoxische fractie in een tumor. Verder groeien EGFRvIII+ tumoren doorgaans sneller en vindt er snellere hergroei van de tumor plaats na bestraling.

We hebben aangetoond dat tumoren met de EGFRvIII mutatie meer autofagie hebben en hiervan ook meer afhankelijk zijn voor hun overleving (hoofdstuk 4). Omdat hypoxie bijdraagt een verminderde prognose, hebben we geprobeerd de hypoxische fractie van een tumor te verlagen door autofagie te remmen met de drug chloroquine. Chloroquine remt autofagie waardoor cellen gevoeliger worden voor stress zoals nutrientstarvatie en hypoxie. Verder hebben we aangetoond in muismodellen dat tumoren beter reageren op bestraling wanneer autofagie wordt geremd. Dit komt waarschijnlijk door het verlagen van de hypoxische fractie. Dit effect is het sterkst bij EGFRvIII+ tumoren. In een retrospectieve studie hebben we aangetoond dat GBM patiënten die gelijktijdig behandeld zijn met chloroquine meer voordeel halen uit de behandeling dan patienten met EGFRvIII negatieve tumoren.

Autofagie breekt cytotoxische componenten af, zoals reactieve zuurstofverbindingproducerende (reactive oxigen species (ROS)) mitochondriën, en wordt daarom beschouwd als een tumorsupressormechanisme. Verschillende studies hebben aangetoond dat autofagie-defecten, door bijvoorbeeld mutaties of deleties van eiwitten in de autofagiemachinerie zoals BECLIN1, bijdragen aan oncogene transformatie van cellen of minsten neoplastische groei. ATG12 is een essentieel eiwitten van de autofagie machinerie en is betrokken bij de lipidatie van de LC3/GABARAP familie. Wij hebben aangetoond dat ATG12 expressie is verloren in ongeveer $40 \%$ van de tumoren in een cohort 
van 428 hoofd-halskanker patiënten (hoofdstuk 5). Uit een ander cohort waarvan de hypoxieachtergrond bekend is, bleek dat ATG12 negatieve tumoren een sterk verlaagde hypoxische fractie hebben. Patiënten met een ATG12 negatieve tumor hebben daardoor een hogere kans op locale tumorcontrole en hogere overlevingskans in stadium 3 en 4 van de ziekte. Opvallend genoeg lijkt deze verlaging van de hypoxische fractie niet te zijn veroorzaakt door een defect in autofagie alleen. Doordat ATG12 ook een positieve regulator van apoptose is, gaan ATG12 negatieve cellen waarschijnlijk dood door andere mechanismen zoals necrose. Het niet naar behoren functioneren van autofagie en ongevoeligheid voor apoptose door het verlies van ATG12, leidt waarschijnlijk tot de vermeerderde necrose die we waarnemen in in vivo studies. Ook zou het zo kunnen zijn dat juist de combinatie van stressfactoren zoals hypoxie en nutriëntstarvatie zorgen voor celdood. Deze combinatie moet nog onderzocht worden.

Naast de rol in degradatie van cellulaire componenten, lijkt autofagie ook betrokken bij vormen van secretie. In Hoofdstuk 6 hebben we uitgebreid literatuuronderzoek gedaan naar hoe autofagie-afhankelijke secretie bijdraagt aan tumorprogressie. Steeds meer onderzoeken laten zien dat autofagie betrokken is bij de secretie van tumorgroei stimulerende factoren, zoals groeifactoren maar ook immuunmodulerende factoren die bijdragen aan het immunosuppressieve karakter van het tumormicromilieu.

Tot deze factoren behoren ook de extracellulair vesicles (EVs). Dit zijn minuscule blaasjes die cellen onder andere gebruiken voor intercellulaire communicatie. Wij hebben aangetoond dat hypoxische cellen een subpopulatie EVs uitscheiden, gekenmerkt door GABARAPL1 expressie op het membraan (GABARAPL1+EVs) (hoofdstuk7). Deze EVs hebben pro-angiogenetische eigenschappen, dat wil zeggen dat ze de vorming van bloedvaten stimuleren dat weer bijdraagt aan tumorgroei. Tevens hebben we aangetoond dat deze GABARAPL1 ${ }^{+} E V$ s zijn te detecteren en verhoogd zijn in het bloed van kankerpatiënten. Doordat GABARAPL1 zich aan de buitenkant van deze EVs bevindt en in circulatie van de patiënt zijn, kunnen hier mogelijke therapieën op gericht worden. We hebben al aangetoond dat de functie van deze GABARAPL1+EVs geblokkeerd kan worden door middel van antilichamen die aan deze EVs binden. Momenteel zijn we bezig met de ontwikkeling van monoclonale antilichamen om de therapeutische werking te onderzoeken in muismodellen.

Naast pro-angiogenetische eigenschappen zijn GABARAPL1+EVs mogelijk betrokken bij de vorming van metastasen (Hoofdstuk 8). In metastaserende muismodellen zijn we in staat om het aantal longmetastasen met $80 \%$ te verminderen wanneer GABARAPL1 wordt uitgeschakeld door middel van een hairpin. Om te bewijzen dat het GABARAPL1+EVs zijn die een rol spelen bij de vorming van deze metastasen is nog verder onderzoek nodig. Wel zijn er aanwijzingen dat GABARAPL1+EVs een rol spelen bij de vorming van de zogenaamde "pre-metastatic niche". Dat zijn gebiedjes elders in het lichaam die worden voorbereid op circulerende tumorcellen nog voordat deze hier kunnen nestelen en uitgroeien tot een metastase. 
Valorisation 
Despite great advanced in cancer research, cancer is one of the leading causes of death in western society. In solid tumours, areas deprived of oxygen (hypoxia) arise due to an aberrant and immature vasculature. Tumour hypoxia is a common characteristic of solid tumours and leads to expansion of tumour cells with a more invasive, metastatic and therapy resistant phenotype, and is therefore a major clinical challenge. The work in this thesis describes mechanisms that hypoxic cancer cells use to sustain their survival and thereby contribute to progression of the disease. Understanding of these mechanisms is important so that hypoxia can be detected and therapies can be directed to target these more resistant cells and improve treatment outcome. As a result of innovative research in the industry and academia, cancer survival rates have increased dramatically over the last decades and have a positive societal impact.

\section{Clinical relevance}

Tumour hypoxia is clinically important as hypoxic tumours show resistance to most cancer therapies and are therefore associated with decreased patient survival. Typically hypoxia reduces the effectiveness of radiotherapy. Depressed oxygen levels in tissue lead to a decrease of long-lived reactive oxygen radicals after $X$ - or $Y$-irradiation that are required for fixed DNA damage of the tumour cell. Consequently, hypoxic cells are three times more resistant to irradiation than well oxygenized cells. Additionally, hypoxic cells are resistant to chemotherapeutic drugs. Typically, chemotherapeutic drugs target rapidly dividing cells. Hypoxia reduces cell proliferation and thereby limits the effects of chemotherapeutic agents. Also the decreased drug delivery due to the aberrant vasculature in the tumour contributes to resistance of chemotherapeutics due to delivery limitations. Also the propagation of metastasis and reduced the effectiveness of surgery are linked to hypoxia. Tumour metastasis is associated with high patient mortality and is responsible for more than $90 \%$ of cancer-related deaths.

Taken together, hypoxia is one of the strongest contributors to patient's poor treatment outcome. Therefore it is highly desired to develop methods to determine hypoxia in a tumour. Such a method can be used as a marker for treatment response but is also required to stratify patients for the right therapy plan and for the development of therapies targeting hypoxia.

Hypoxia cannot be determined anatomically, as hypoxia is independent of tumour size, grade and stage. Currently, there are no markers for tumour hypoxia used in the clinic for diagnostic or treatment purposes. Invasive polarographic electrodes are the 'gold standard' for assessing tumour hypoxia. However due to the heterogeneous and dynamic properties of hypoxia, multiple samples must been taken in the diseased tissue, and are due to the high burden for the patient never used. Determining hypoxia by imaging markers, such as HX4 scanning or others have not shown adequate specificity or do not correlate with hypoxia at all. Other described methods are the use of deter- 
mining osteopontin in patient fluids. However due the low elevation of this marker in hypoxic tumours compared to healthy subjects, this method is of limited use when applied to a single patient. Taken together, methods to determine hypoxia in a tumour are limited and are not implemented in current treatment plans, but also to stratify patients for hypoxia-targeted therapies hypoxia markers are highly desired.

\section{Improvement for healthcare}

The work described in this thesis can be exploited to modify tumour hypoxia to improve therapy outcome, but also to develop targeted therapies and prognostic biomarkers.

In chapter 5, findings are described that ATG12 could serve as a predictor for tumour hypoxia and a tumour control in head and neck cancer patients. We found that in 25$40 \%$ of patients with head and neck cancer, tumours lack ATG12 expression. The loss of ATG12 expression correlates with a decreased hypoxic fraction in the tumour. Strikingly, patients with ATG12 'negative' tumours show improved local and loco-regional control. Therefore, ATG12 expression can be used as a hypoxia predictor and be implemented in pathohistological analysis after biopsies. Such a marker can contribute to stratify patients for clinical trials investigating hypoxia targeted therapies. I.e. ATG12 negative tumours are expected to be less sensitive to these therapies and should therefore be excluded from these trials. Because ATG12 expression is lost in a substantial group of head and neck cancer patients, institutes investigating the effectiveness of hypoxia targeting therapies in clinical trials should be aware of this phenomenon before including patients in their trials. To investigate the effectiveness of hypoxia targeted therapies, stratification of patients with hypoxic tumours is required. The finding that ATG12 negative tumours have almost no hypoxic fraction could contribute to improved outcome of these trials.

In chapter 7 we describe our findings that hypoxic cells secrete a subset of extracellular vesicles that is marked by GABARAPL1 expression on their surface. This finding provides means for both diagnostic and therapeutic applications and marketable products: Since GABARAPL1+EVs are released by hypoxic cells and are detectable in circulation of patients, GABARAPL1+EVs can be used as a hypoxia marker. We already showed that GABARAPL1+EVs are detectable and elevated in the blood of a small group $(n=10)$ of colorectal, glioblastoma and small cell lung cancer patients. Currently our group is investigating if the number of circulating GABARAPL1+EVs correlate with the degree of hypoxia in a tumour to evaluate if we can determine the amount hypoxia. Enzymelinked immunosorbent assays (ELISA) are suitable assays to determine macromolecular species in fluids and are frequently used as a diagnostic tool. Currently our group is developing ELISA assays to detect GABARAPL1 ${ }^{+} E V$ s in the blood of cancer patients. Such 
a GABARAPL1+EV ELISA can be translated in a marketable product and could be exploited as such. A GABARAPL1+EV ELISA could provide information about the degree of hypoxia in a 'bench test' which is more desirable than invasive detection or expensive scanning methods which also require specialized personnel. Currently hypoxia activated pro-drugs (HAPs) are in development to specifically target hypoxic cells in a tumour. Tools to determine if a tumour is hypoxic are highly desired for patient stratification for these clinical trials.

The finding that GABARAPL1 is expressed on EVs also has therapeutic applications. Since GABARAPL1 is expressed on the surface of hypoxic EVs, it is accessible for targeting, for instance with antibodies. We already showed in chapter 8 that inhibition of GABARAPL1 with hairpin RNA's decreased metastasis formation by $80 \%$ in a metastatic breast cancer model in mice. Additionally, targeting GABARAPL1 resulted in decreased angiogenesis, tumour growth and tumour regrowth after irradiation and importantly, GABARAPL1 $1^{+} E V$ s are detectable and elevated in the circulation of cancer patients compared to healthy controls (chapter 7).

Therefore, we hypothesize that targeting GABARAPL1+EVs in the patient's circulation by GABARAPL1 targeting antibodies can reduce the formation of metastasis, but also might affect growth of the primary tumour; two important goals to achieve in patients. In chapter 7 we showed in a proof of concept experiment that we are able to block GABARAPL1 ${ }^{+} E V$ with antibodies targeting GABARAPL1 in vitro and thereby inhibiting their pro-angiogenic properties. Extrapolated to patients, this could include that this approach might prevent the formation of a pre-metastatic niche, and thereby prevent the development of metastasis.

These in vitro experiments were done with commercially available polyclonal antibodies. For therapeutic applications, typically monoclonal antibodies are used. Most used antibody subtypes are IgG1, 2 or 4, due to their long half-life in circulation (up to 21 days). We already have developed GABARAPL1 targeting monoclonal antibodies to further explore and validate this approach in in vitro and in vivo studies.

Since the use of the first monoclonal antibody in 1986, the market for these biopharmaceutical drugs has grown significantly. At present, 47 antibodies have been approved in the US or Europe. Annually, 4 new antibodies achieve marked approval, leading to an estimated world-wide sale of nearly \$125 billion in 2020 [1]. However, currently there are no therapeutics on the marked or clinical trials investigating drugs targeting endogenous extracellular vesicles. In that perspective, this method could lead to the development of a novel and innovative anti-cancer therapy. The method to target EVs, and more specifically GABARAPL1+EVs, to prevent the formation of metastasis is described in a patent in Europe and the US (Keulers \& Rouschop, patent number W02015121295A1). To further develop this approach and translate this into a possible treatment we are looking for research partners to support further (co-)-development and clinical proof of concept treatment. Such collaboration could lead to a university spin-off company to explore its clinical feasibility and efficacy. 
GABARAPL1+EV targeting antibodies could be applied as a neoadjuvant therapy on patients with solid, and thus hypoxic, tumours. It needs to be empirically determined if GABARAPL1+EVs indeed are secreted by most solid tumours, but it is expected that most tumours release GABARAPL1 ${ }^{+} E V$ s. Most cancer related deaths are attributed the metastatic disease. Typically, distant metastases are frequently unresponsive to existing therapies and are treated systemically with palliative intent. Preventing the formation of metastasis could improve the life expectancy of many patients.

Tumour hypoxia contributes to poor treatment outcome in several cancer types. Therefore, reducing the hypoxic fraction in a tumour is highly desired. Attempts to modulate the hypoxic fraction were primarily focussed on increasing oxygen supply to the tumour. A different approach is alleviating hypoxia by targeting cellular mechanisms that use cells for their survival, such as autophagy.

Chloroquine is originally marketed as an anti-malaria drug, but is also used in the treatment of rheumatoid arthritis and lupus-associated arthritis. In addition to these properties, chloroquine is a potent autophagy inhibitor. Previously we showed that chloroquine sensitizes cells to hypoxia, reduces the hypoxic fraction and sensitizes xenograft tumours to radiotherapy in mice [2].

In chapter 4 we show that EGFRvIII expression elevates the activation of autophagy during hypoxia. The mutant form of EGFR, EGFRvIII is frequently observed in glioblastoma (50-60\% of patients with EGFR amplification) and is associated with increased therapy resistance and poor prognosis. A retrospective study indicated that patients with EGFRvIII positive tumours benefit most of concurrent chloroquine treatment (median survival form 3 to 15 months).

Currently, a phase I clinical trial is ongoing in our institute (clinical trial NCT02378532) to determine the maximum tolerated dose of chloroquine with concurrent radiotherapy and temozolomid. So far, this trial shows promising results. To determine if chloroquine can be repurposed as a drug in oncology and considered as an additional treatment for GBM patients, additional clinical trials are required to investigate efficacy, effectiveness and safety.

\section{References}

1. Ecker, D.M., S.D. Jones, and H.L. Levine, The therapeutic monoclonal antibody market. mAbs, 2015. 7(1): p. 9-14.

2. Rouschop, K.M., et al., The unfolded protein response protects human tumor cells during hypoxia through regulation of the autophagy genes MAP1LC3B and ATG5. The Journal of clinical investigation, 2010. 120(1): p. 127-41. 

Acknowledgments / Dankwoord 
Leuk, zo'n baantje van 4 maanden! Even tijdens je master wat extra ervaring opdoen in het lab! In het lab bleek het echter veel leuker dan in de collegebanken, dus het was al snel duidelijk dat het niet bij die 4 maanden zou blijven. Een paar jaar als analist volgden, doorgestroomd naar promovendus, en nu ligt hier het resultaat! Dat doe je natuurlijk niet alleen, zo'n proefschrift. Er hebben door de jaren heen heel wat mensen, direct of indirect, aan bijgedragen die ik graag wil bedanken.

Kasper, al heel wat jaren werken we nu samen. En dit is eindelijk eens een moment om je te bedanken voor alles wat je de afgelopen jaren voor me hebt gedaan. Ik wil je bedanken voor het vertrouwen dat je van begin af aan in mij hebt gehad. Het heeft enorm bijgedragen aan wie ik nu ben en hoe ik onderzoek doe. Ik heb veel respect voor jou als persoon en als wetenschapper (ook al lijkt dat niet altijd zo;)). Je vernieuwende ideeën en je eeuwige optimisme zijn altijd een inspiratie voor me. Je deur staat letterlijk altijd voor me open, wat vaak resulteert in lange brainstormsessies over waarom we in godsnaam aan dat eiwit zijn gaan werken en hoe we de volgende experimenten gaan doen. Kasper, dankjewel!

$\underline{\text { Kim, }}$ je was mijn steun en toeverlaat vanaf mijn eerste stage, lang geleden. Met jou in de buurt, wist ik dat het altijd allemaal wel goed kwam. Ik wil je bedanken voor je collegialiteit, je kennis, je jaarlijkse carnavalsvibe en alle hulp die je hebt gegeven aan onze projecten, maar ook voor het in goede banen leiden van alles op en rondom het lab. Met jou in de buurt is het altijd een gezellige boel!

$\underline{\text { Jan }}$, bij jou is het allemaal begonnen. Ik weet nog goed dat ik bij een presentatie tijdens mijn studie direct was geboeid door je werk aan bacteriën die therapieën naar de tumor brengen. Een zeer interessante stage in je groep volgde, met als mededeling dat ik altijd terug mocht komen. Zo geschiedde. Jan, je bent altijd een inspirator voor me geweest. Als jij praat, luister ik.

Marc, bedankt voor al je input en de leuke gesprekken naast het werk over fietsen, fietstochten, gitaren, muziek en sterke kraakverhalen! De verse koffie heeft me elke ochtend weer op de been geholpen!

Graag wil ik de beoordelingscommissie, Frans Ramaekers, Esther Nolte-'t Hoen, Raymond Schiffelers, Patrick Schrauwen en Lotte Wieten bedanken voor de tijd die jullie hebben genomen om mijn proefschrift te beoordelen!

Marike, met jou als mijn roomie was het altijd gezellig en had ik altijd een opgeruimd bureau! Soms weet ik nog steeds niet of jij nou langs bent geweest of dat het toch de poetsvrouw was. Bedankt voor je collegialiteit, je vriendelijkheid en al die ommetjes naar de koffieautomaat!

En dan is er Marijke, bekend om haar slechte muzieksmaak, maar voornamelijk om haar goede vragen op het goede moment. Een gewaardeerd lid van onze autofagieclub 
en meer dan een collega alleen. Altijd in voor een feestje! Bedankt voor alle gezelligheid op het lab en daarbuiten!

Ik wens jullie beiden heel veel succes!

Hanneke, inmiddels onze club verlaten, maar de bijdrage die je hebt geleverd aan onze projecten zal ik niet vergeten! Honderden westernblotjes en heel wat uurtjes hebben we samen tumoren zitten meten in DM2. Bedankt voor je inzet! Barry, je was altijd een van de steunpilaren van het lab. Ik kon altijd bij je terecht met vragen en je was altijd bereid te helpen en mee te denken. Op de een of andere manier resulteerde het wel altijd in schunnige praat! Zelfs het Duits werd hierbij niet geschuwd! Bedankt voor alles Barry! Heel veel succes in de toekomst!

En van schunnige praat is het een kleine stap naar Roger en Marco Uit het oog, maar niet uit het hart! Met jullie in de buurt was het altijd tijd voor ongein, op het lab, maar ook daarbuiten! Ik wens jullie veel succes met jullie carrière!

Dan zijn er nog de PhD roomies, Damiënne \& Alexander, Michael, Lorena, Judith, Jonathan, Veronica, Eloy, Raymon en Venus. Een kantoor waar menig soapschrijver zijn hart aan op kan halen! Bedankt voor de leuke tijd, de ritjes naar de koffie de gezellige avonden en soms lange nachten! De tijd is gekomen dat jullie op zoek moeten naar een nieuw stamhoofd. Veel succes met het afronden van jullie PhDs!

Nicolle, met jouw in de buurt was er altijd iets te beleven! De soepjes en de middagwandelingetjes waren altijd een fijn moment om even af te schakelen! Je wordt gemist! Arjan, Dr. Groß!, bedankt voor al je verhalen, je enthousiasme en je ideeën!

Analisten zijn de ruggengraat van een lab! Bedankt Natasja, Rianne, Jolanda en Lydie, voor jullie inzet, hulp bij in vivo studies en het draaiend houden van het lab. Rianne, het recept van mijn geheime cocktail heb je nog tegoed;)! Ook Nicole Bitsch bedankt voor alle hulp bij de in vivo experimenten! Ook Carla, die het toch maar altijd even regelt op de achtergrond, bedankt voor alles!

Ook Ludwig, Ala en alle andere collega's bij Maastro lab, bedankt voor jullie collegialiteit en input wanneer dat nodig was!

Veel van dit werk was niet mogelijk geweest zonder de expertise in Utrecht! Marca, bedankt voor de fijne samenwerking en de introductie in de wereld van de EVs! Met Sten zijn er heel wat uurtjes, wat zeg ik, dagen, doorgebracht in het labje bij de Influx, maar het heeft heel wat mooie data en verhalen opgeleverd! Dank je Sten, en veel succes met je postdoc in Cambridge!

Als we het over fijne samenwerkingen hebben, kom je al snel uit in Nijmegen waar ze het analyseren van tumoren tot in de fijnste puntjes beheersen, bedankt Paul Span, Johan Bussink en Hans Peters voor alle analyses en al het werk wat jullie voor ons gedaan hebben! 
Als je hulp nodig hebt met welke vorm van microscopie dan ook, moet je bij Hans Duimel, Helma Kuijpers, Marc van Zandvoort, en Carmen López Iglesias zijn! Ze leggen het je tot in het kleinste detail uit en zijn altijd bereid te helpen!

Dan zijn er nog mijn stagestudenten Imke, Laura en Britta die naast hun gezelligheid en uitbundige treinritten ook nog een substantiële bijdrage hebben geleverd aan dit onderzoek. Succes met jullie PhDs en verdere carrière, ik heb er alle vertrouwen in!

Veerle Melotte en Alexander Koch, bedankt voor jullie bijdrage aan het hoofdstuk over ATG12!

Er moet ook genoeg afleiding zijn! Met mijn maatjes Roel-Germ, Ronald, Eric, Nils, Sebastiaan en Sander in de buurt is dat nooit een probleem! Bedankt voor alle lol, maffe avonden, alle biertjes en jullie vriendschap!

Mijn lieve ouders, door jullie was mijn interesse in de biologie al vroeg gewekt; plantjes, vlindertjes en andere beestjes, alles werd thuis gekweekt en bestudeerd. Op mijn achtste kreeg ik al mijn eigen microscoop en ik was trots op mijn trofeeën-rek vol samen uitgekookte dierenskeletjes en gevonden fossielen. Ook al was ik tijdens mijn studiejaren soms wat afgeleid, jullie hebben altijd vertrouwen in mij gehad. Zonder jullie grote hulp was het bovendien niet gelukt om ons huis te verbouwen en te promoveren tegelijk. Bedankt voor jullie onvoorwaardelijke steun en hulp en dat jullie me altijd in alles gestimuleerd hebben!

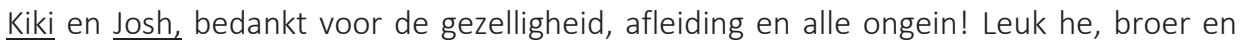
zus!

Charles en Gerrie, bedankt voor jullie nimmer aflatende interesse in mijn werk en onderzoek, al jullie hulp en steun en vooral de gezellige tijd de afgelopen jaren!

Lieve Anne, met jou in de buurt is het altijd het leukst! Dank je voor je onvoorwaardelijke steun, je liefde en dat je me altijd weer op de been hielp wanneer het even tegenzat. Met ons huisje zo goed als klaar, jij op het punt om te beginnen met je opleiding tot huisarts, zie ik de toekomst met vertrouwen tegemoet $(;)$

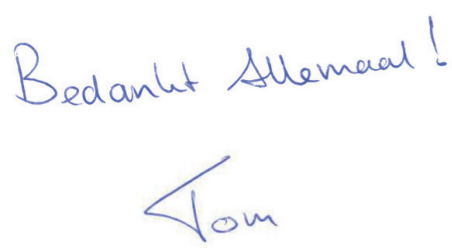


Curriculum Vitae 


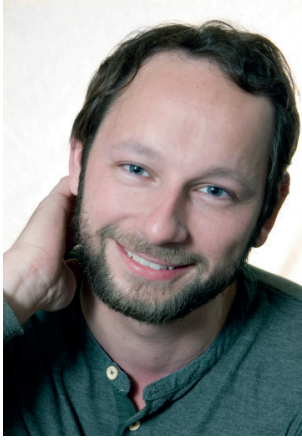

Tom Keulers was born on February $28^{\text {th }} 1985$ in Kerkrade, the Netherlands. In 2003 he obtained his secondary school degree at College Rolduc in Kerkrade. He then performed his bachelor studies (HLO) at Hogeschool Zuyd, Heerlen. In 2007 he obtained his bachelor's degree of applied sciences with a specialization in biochemical research. He continued his master studies Biopharmaceutical sciences at Leiden University, the Netherlands. During the first year of his master program he did an internship at the department of Biopharmacy, where he worked on apoptosis in atherosclerotic plaques. He continued his master program in the group of Dr. Kasper Rouschop at the department of radiotherapy (MaastroLab in Maastricht) where he laid the foundation for his PhD later in that group. After completing his master's degree with a specialization in drug-delivery technology and biopharmaceutics in 2012, he started as a PhD student in Dr. Rouschop's group. During this period he worked on the role of autophagy and extracellular vesicles in the hypoxic tumour microenvironment, which led to several publications and the discovery of GABARAPL1+EVs. This work was selected as 'late breaking abstract' at the international society for extracellular vesicles (ISEV), proffered presentations at international conferences, travel grands and was awarded with "best poster prize" at the European radiation research society (ERRS) 2016 and led to an international patent.

Recently Tom is granted the Kootstra talent fellowship to continue his research at the department of Radiotherapy at Maastricht University. 


\section{List of Publications}


ATG12 expression predicts tumour hypoxia and tumour control in HNSCC

Tom G. Keulers, Alexander Koch, Hanneke Peeters, Kim G.M. Savelkouls, Nicole Bitsch, Roel G. Wanders, Dennis Roop, Johan Bussink, Veerle Melotte, Kasper M.A. Rouschop.

(In preparation)

Extracellular Vesicles as transmitters of hypoxia tolerance in solid cancer

Marijke I Zonneveld, Tom GH Keulers and Kasper MA Rouschop

(submitted)

GABARAPL1 is required for extracellular vesicle secretion during hypoxia

Tom G. Keulers, Sten Libregts, Marco Schaaf, Hanneke Peeters, Kim Savelkouls, Johan Bussink, Hans Duimel, Marc Vooijs, Barry Jutten, Marca Wauben, Kasper Rouschop (submitted)

EGFRvIII expression triggers a metabolic dependency and therapeutic vulnerability sensitive to autophagy inhibition.

Jutten B, Keulers TG, Peeters HJM, Schaaf MBE, Savelkouls KGM, Compter I, Clarijs R, Schijns OEMG, Ackermans L, Teernstra OPM, Zonneveld MI, Colaris RME, Dubois L, Vooijs MA, Bussink J, Sotelo J, Theys J, Lammering G, Rouschop KMA.

Autophagy. 2018;14(2):283-295. doi: 10.1080/15548627.2017.1409926

Leukocyte Bim deficiency does not impact atherogenesis in Idlr -/- mice, despite a pronounced induction of autoimmune inflammation.

Temmerman L, Westra MM, Bot I, van Vlijmen BJM, van Bree $N$, Bot $M$, Habets $K L L$, Keulers TG, van der Vlag J, Cotter TG, van Berkel TJC, Biessen EAL.

Sci Rep. 2017 Jun 8;7(1):3086. doi: 10.1038/s41598-017-02771-4.

LC3/GABARAP family proteins: autophagy-(un)related functions.

Schaaf MB*, Keulers TG*, Vooijs MA, Rouschop KM.

FASEB J. 2016 Dec;30(12):3961-3978. Review.

Autophagy-Dependent Secretion: Contribution to Tumor Progression.

Keulers TG*, Schaaf MB*, Rouschop KM.

Front Oncol. 2016 Nov 25;6:251. eCollection 2016. Review.

GABARAPL1 is required for increased EGFR membrane expression during hypoxia.

Keulers TG, Schaaf MB, Peeters HJ, Savelkouls KG, Vooijs MA, Bussink J, Jutten B, Rouschop KM.

Radiother Oncol. 2015 Sep;116(3):417-22. doi: 10.1016/j.radonc.2015.06.023. Epub 2015 Jul 8. 
Canonical autophagy does not contribute to cellular radioresistance.

Schaaf MB, Jutten B, Keulers TG, Savelkouls KG, Peeters HJ, van den Beucken $T$, van Schooten FJ, Godschalk RW, Vooijs M, Rouschop KM.

Radiother Oncol. 2015 Mar;114(3):406-12. doi: 10.1016/j.radonc.2015.02.019

EGFR overexpressing cells and tumors are dependent on autophagy for growth and survival.

Jutten B, Keulers TG, Schaaf MB, Savelkouls K, Theys J, Span PN, Vooijs MA, Bussink J, Rouschop KM.

Radiother Oncol. 2013 Sep;108(3):479-83. doi: 10.1016/j.radonc.2013.06.033

The autophagy associated gene, ULK1, promotes tolerance to chronic and acute hypoxia. Schaaf MB, Cojocari D, Keulers TG, Jutten B, Starmans $M H$, de Jong MC, Begg AC, Savelkouls KG, Bussink J, Vooijs M, Wouters BG, Rouschop KM.

Radiother Oncol. 2013 Sep;108(3):529-34. doi: 10.1016/j.radonc.2013.06.015.

PERK/elF2 a signaling protects therapy resistant hypoxic cells through induction of glutathione synthesis and protection against ROS.

Rouschop KM, Dubois LJ, Keulers TG, van den Beucken T, Lambin P, Bussink J, van der Kogel AJ, Koritzinsky M, Wouters BG.

Proc Natl Acad Sci U S A. 2013 Mar 19;110(12):4622-7. doi: 10.1073/pnas.1210633110

Deregulation of cap-dependent mRNA translation increases tumour radiosensitivity through reduction of the hypoxic fraction.

Rouschop KM, Dubois L, Schaaf MB, van den Beucken T, Lieuwes N, Keulers TG, Savelkouls KG, Bussink J, van der Kogel AJ, Koritzinsky M, Wouters BG.

Radiother Oncol. 2011 Jun;99(3):385-91. doi: 10.1016/j.radonc.2011.05.047

The unfolded protein response protects human tumor cells during hypoxia through regulation of the autophagy genes MAP1LC3B and ATG5.

Rouschop KM, van den Beucken T, Dubois L, Niessen H, Bussink J, Savelkouls K, Keulers T, Mujcic H, Landuyt W, Voncken JW, Lambin P, van der Kogel AJ, Koritzinsky M, Wouters BG. J Clin Invest. 2010 Jan;120(1):127-41. doi: 10.1172/JCI40027.

Autophagy is required during cycling hypoxia to lower production of reactive oxygen species.

Rouschop KM, Ramaekers CH, Schaaf MB, Keulers TG, Savelkouls KG, Lambin P, Koritzinsky $M$, Wouters $B G$.

Radiother Oncol. 2009 Sep;92(3):411-6. doi: 10.1016/j.radonc.2009.06.029 


\section{Patents}

Method for determining the level of hypoxia in a tumor (EP3105593A1 \& US20170176445A1).

K.M.A Rouschop \& T.G.H. Keulers

*contributed equally 


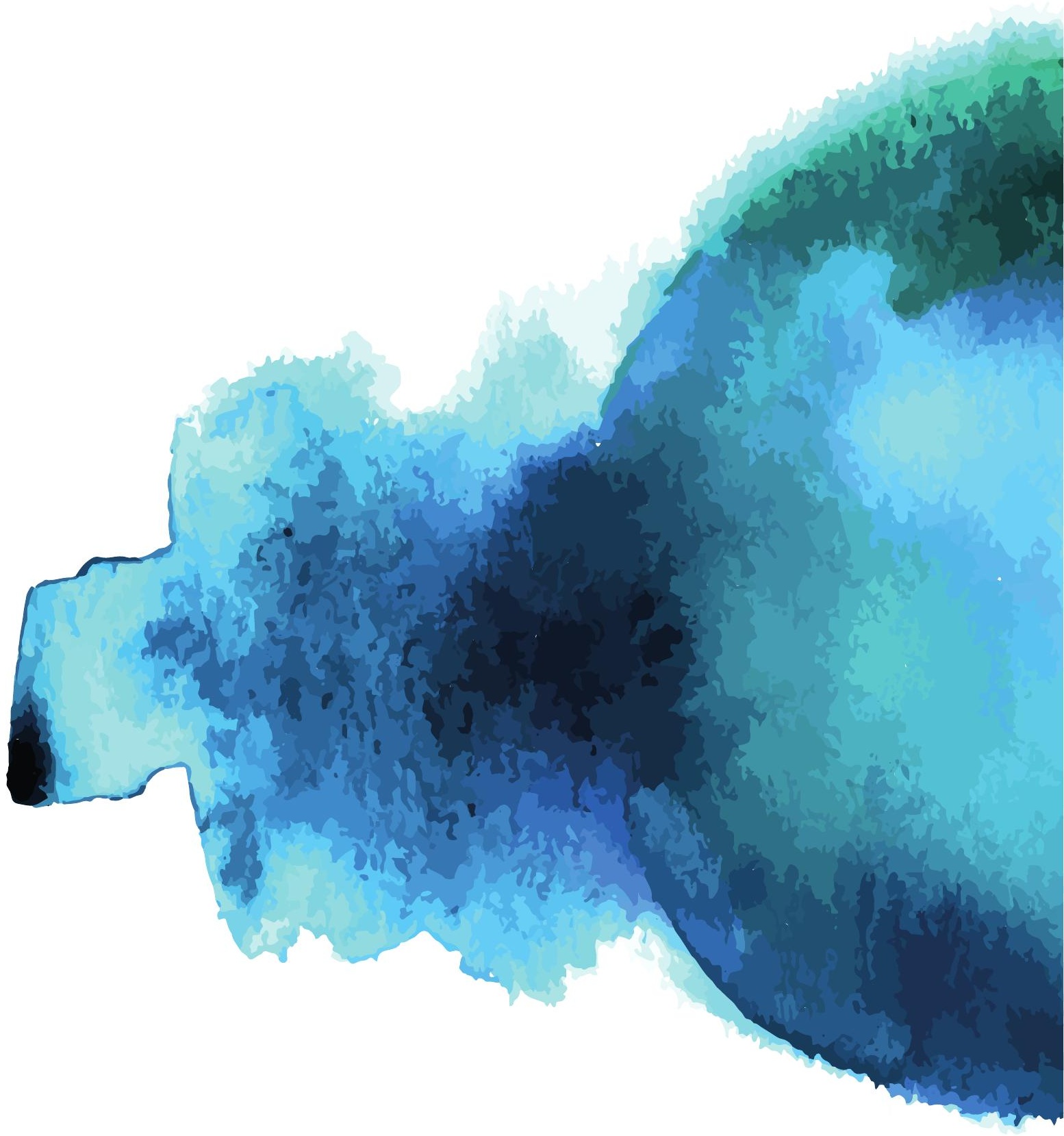

